\title{
Angst und Leistung im Rahmen der Katastrophentheorie - Untersuchungen zum optimalen Erregungsniveau bei Fallschirmspringern
}

\author{
Dissertation \\ zur Erlangung des sozialwissenschaftlichen Doktorgrades \\ der Sozialwissenschaftlichen Fakultät der Universität Göttingen \\ vorgelegt von \\ Steffen Schiedek \\ aus Göttingen
}

Göttingen, im Oktober 2003 
1. Gutachter: Prof. Dr. Arnd Krüger

2. Gutachter: Prof. Dr. Peter Faßheber

Tag der mündlichen Prüfung: 01.06.2004 


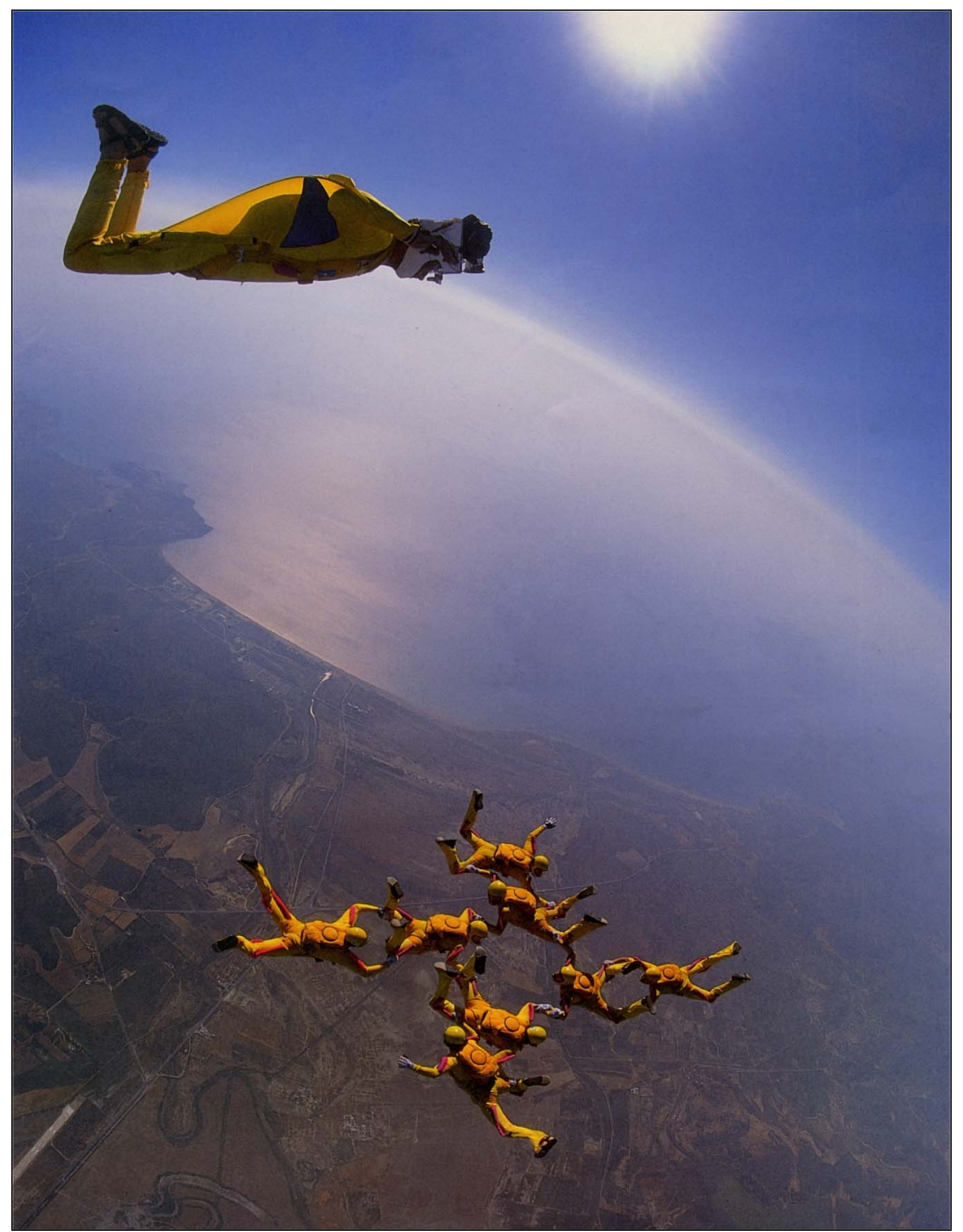

Der Deutschland-Achter in der Luft über der Türkei während der WM 1997 (Foto: Bruno Brokken).

The exhilaration of flying is too keen, the pleasure too great, for it to be neglected as a sport. 


\section{Inhaltsverzeichnis}

ABBILDUNGSVERZEICHNIS V V

TABELLENVERZEICHNIS VII

ABKÜRZUNGEN

$\begin{array}{lll}\text { ABSTRACT } & \text { IX }\end{array}$

I EINLEITUNG

II THEORETISCHER TEIL

1 ANGST - EIN BEGRIFF AUS UNTERSCHIEDLICHEN SICHTWEISEN

1.1 DEFINITIONEN VON ANGST 4

1.2 Physiologische Betrachtung VON ANGST 9

1.3 BewÄLtigung VON ANGST - COPING 13

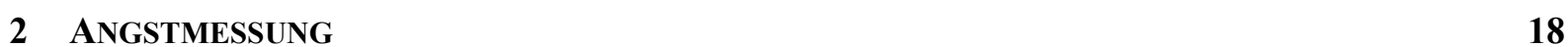

2.1 ANGST-INDIKATOREN 18

2.2 MeSSMETHODEN DER ANGST 19

$3 \quad$ ANGST UND SPORTLICHE LEISTUNG

3.1 AngSt-Leistungs-Modelle 27

3.2 WEITERE EINFLUSSGRÖSSEN 35

3.3 Welches ANGST-LeistungS-Modell IST DAS RICHTIGE? 46

4 KATASTROPHENTHEORIE $\quad 48$

4.1 VOM CHAOS ZUR KATASTROPHENTHEORIE

4.2 EINIGE ELEMENTARE KATASTROPHENFORMEN 53

4.3 ANGST UND LEISTUNG AUS KATASTROPHENTHEORETISCHER SICHT 60

4.4 STATISTISCHE AUSWERTUNG IM RAHMEN DER KATASTROPHENTHEORIE 65

5 EINORDNUNG DES FALLSCHIRMSPRINGENS

$\begin{array}{lll}5.1 & \text { DIE FALLSCHIRMSPORTDISZIPLINEN } & 74\end{array}$

$\begin{array}{lll}5.2 & \text { FREIFALLFORMATIONSSPRINGEN } & 78\end{array}$ 
III EMPIRISCHER TEIL

1 Fragestellungen $\quad 88$

2 HYPOTHESENBILDUNG $\quad 91$

3 UNTERSUCHUNGSMETHODIK

3.1 BeschreIBUNG DER PROBANDENGRUPPE 92

3.2 UNTERSUCHUNGSVERLAUF 96

4 KRITIK AN DER METHODIK

4.1 SYSTEMATISCHE ASPEKTE 99

4.2 KRITIK AN DEN VERWENDETEN FRAGEBÖGEN 105

5 UNTERSUCHUNGSERGEBNISSE

$\begin{array}{lll}5.1 & \text { DESKRIPTIVE ERGEBNISSE } & 108\end{array}$

5.2 Statistische ERGEBNisSE 120

5.3 DISKUSSION DER ERGEBNISSE 136

6 Praxisbezogene Relevanz der Ergebnisse 142

IV ZUSAMMENFASSUNG UND AUSBLICK

$\underline{V}$ LITERATUR 146

\begin{tabular}{ll} 
ANHANG & 162 \\
\hline
\end{tabular}

VERWENDETE FRAGEBÖGEN 163

Ausgabe DEs Programms GEMCAT II: ANGEPASSTE SPITZENKATASTROPHENFLÄCHEN FÜR DIE UNTERSUCHUNGSGRUPPEN $\quad 175$

AUSGABE DES PROGRAMMS GEMCAT II: ANGEPASSTE SCHMETTERLINGSKATASTROPHENFLÄCHEN FÜR DIE UNTERSUCHUNGSGRUPPEN 184

FREIFALLFORMATIONSPROGRAMM 4ER-DISZIPLIN

FREIFALLFORMATIONSPROGRAMM 8ER-DISZIPLIN

\begin{tabular}{ll} 
LEBENSLAUF & 195 \\
\hline
\end{tabular} 


\section{Abbildungsverzeichnis}

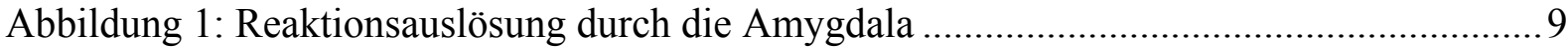

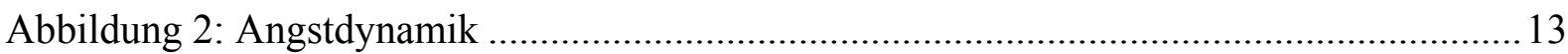

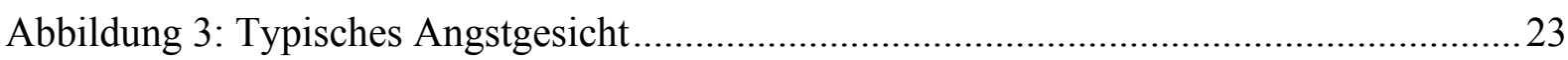

Abbildung 4: Angst/Leistung bei Drive- und umgekehrter U-Theorie ..................................29

Abbildung 5: Additive Darstellung somatischer (umgekehrtes-U) und kognitiver (negativ

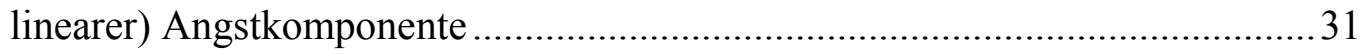

Abbildung 6: Multidimensionale angstbasierende Zone des optimalen Funktionsniveaus ..... 33

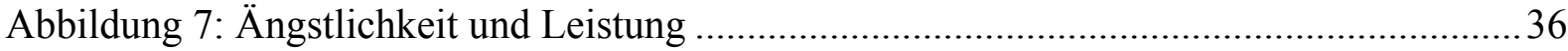

Abbildung 8: Aktivierung, Aufgabenschwierigkeit und Leistung ........................................ 42

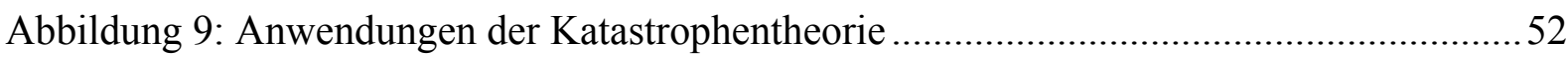

Abbildung 10: Potentialfunktion der Faltenkatastrophe ....................................................5

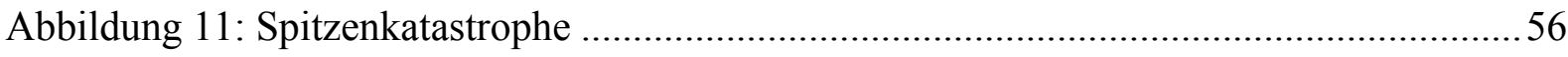

Abbildung 12: Eigenschaften der Spitzenkatastrophe ........................................................58

Abbildung 13: Schmetterlingskatastrophe mit Verschiebungsfaktor $c=0$ und Schmetterlingsfaktor $d=-1$ aus zwei verschiedenen Perspektiven ................59

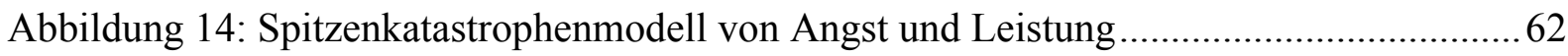

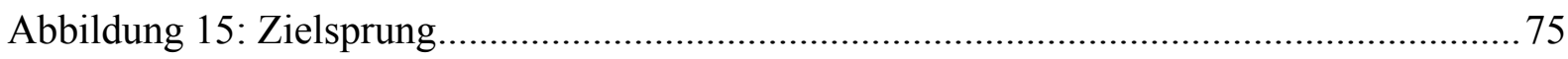

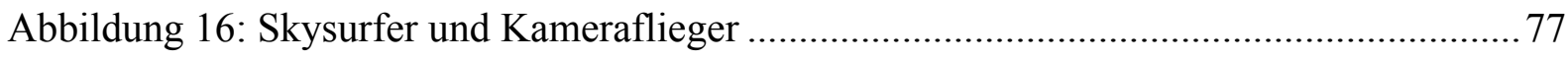

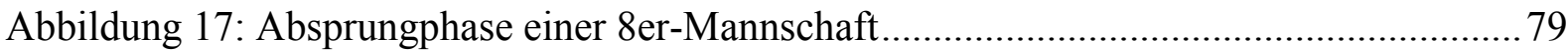

Abbildung 18: Blockübergang 22 von „Old Bone“ zu „Compressed Stairstep Diamonds“

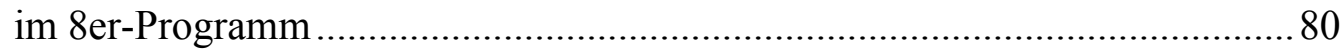

Abbildung 19: 8er-Mannschaft und Kameraflieger beim Formationsspringen....................... 80

Abbildung 20: Blockübergang Nr. 22 der amerikanischen 8er-Nationalmannschaft ..............87

Abbildung 21: Histogramme von Alter und Sprungerfahrung der Gruppe 1 mit niedriger

Eigenschaftsangst.

Abbildung 22: Histogramme von Alter und Sprungerfahrung der Gruppe 2 mit mittlerer

Eigenschaftsangst

Abbildung 23: Histogramme von Alter und Sprungerfahrung der Gruppe 3 mit hoher

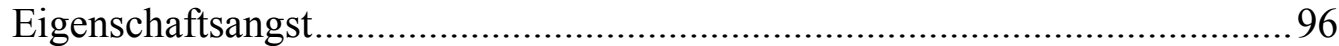

Abbildung 24: Absetzflugzeug DHC-6 „Twin Otter“ während der WM 1999.......................97

Abbildung 25: Normalverteilungsplot: STAI in deutscher und englischer Fassung.............. 107 
Abbildung 26: Normalverteilungsplot: CSAI-2 in deutscher und englischer Fassung 107

Abbildung 27: Durchschnittliche Herzfrequenz der 4er-Formationen in Training und Wettkampf.

Abbildung 28: Durchschnittliche Herzfrequenz der 8er-Formationen in Training und Wettkampf.

Abbildung 29: Herzfrequenz des Probanden AnK-Grou4-D in Training und Wettkampf .... 111

Abbildung 30: Interaktionsplot der Ängstlichkeitsgruppen: Fragebogenwerte und physiologische Aktivierung

Abbildung 31: Interaktionsplot der Selbstwirksamkeitsgruppen: Fragebogenwerte und physiologische Aktivierung

Abbildung 32: Interaktionsplot der Erfahrungsgruppen im Freifallformationsspringen:

Fragebogenwerte und physiologische Aktivierung

Abbildung 33: Korrelation von somatischer Angstkomponente und physiologischer

Aktivierung

Abbildung 34: Mittelwerte der Angstkomponenten in Abhängigkeit des zeitlichen

Sprungverlaufes

Abbildung 35: Katastrophenflächen für unterschiedliche Eigenschaftsangst 125

Abbildung 36: Kontrollebene für Gruppen unterschiedlicher Eigenschaftsangst.

Abbildung 37: Katastrophenflächen für unterschiedliche Selbstwirksamkeits-

überzeugung

Abbildung 38: Kontrollebene für Gruppen unterschiedlicher Selbstwirksamkeitsüberzeugung

Abbildung 39: Katastrophenflächen für unterschiedliche Erfahrung....

Abbildung 40: Kontrollebene für Gruppen unterschiedlicher Erfahrung. 


\section{Tabellenverzeichnis}

Tabelle 1: Merkmale von Angst und Furcht.

6

Tabelle 2: Mittlere Hormon- und Stoffwechselproduktkonzentration im Blut zu verschiedenen Zeiten beim Fallschirmspringen.

Tabelle 3: Optimale Erregungszustände für verschiedene Sportarten.................................... 43

Tabelle 4: Die sieben elementaren Katastrophenformen nach ТНОм ...................................51

Tabelle 5: Im Katastrophenmodell vorhergesagte Leistungsniveaus bei interaktiven

Effekten von physiologischer Erregung und kognitiver Angstkomponente .............68

Tabelle 6: Die Probanden der Gruppe 1: Niedrige Ängstlichkeit ............................................93

Tabelle 7: Die Probanden der Gruppe 2: Mittlere Ängstlichkeit............................................. 94

Tabelle 8: Die Probanden der Gruppe 3: Hohe Ängstlichkeit............................................... 95

Tabelle 9: Mittelwerte und Standardabweichungen der Ängstlichkeitsgruppen:

Fragebogenwerte und physiologische Aktivierung. .......................................... 112

Tabelle 10: Mittelwerte und Standardabweichungen der Selbstwirksamkeitsgruppen:

Fragebogenwerte und physiologische Aktivierung. ......................................... 114

Tabelle 11: Mittelwerte und Standardabweichungen der Erfahrungsgruppen:

Fragebogenwerte und physiologische Aktivierung.

Tabelle 12: Korrelationskoeffizienten $r$ von Angstkomponenten mit der Leistung in den Ängstlichkeitsgruppen

Tabelle 13: Koeffizienten und Fehler der angepassten Spitzenkatastrophenmodelle in den Ängstlichkeitsgruppen

Tabelle 14: Koeffizienten und Fehler der angepassten Schmetterlingskatastrophenmodelle in den Ängstlichkeitsgruppen

Tabelle 15: Koeffizienten und Fehler der angepassten Spitzenkatastrophenmodelle in den Gruppen unterschiedlicher Selbstwirksamkeitsüberzeugung.

Tabelle 16: Koeffizienten und Fehler der angepassten Spitzenkatastrophenmodelle in den unterschiedlichen Erfahrungsgruppen. 


\section{Abkürzungen}

\begin{tabular}{|c|c|}
\hline Anm. & Anmerkung \\
\hline $\mathrm{cm}$ & Zentimeter \\
\hline CSAI-2 & Competitive State Anxiety Inventory-2 \\
\hline DFV & Deutscher Fallschirmsportverband \\
\hline EEG & Elektroenzephalogramm \\
\hline engl. & englisch \\
\hline et al. & et altera \\
\hline e.V. & eingetragener Verein \\
\hline$\phi$ & quadrierte Abweichungssumme (squared sum of residuals) \\
\hline FAI & Fédération Aéronautique Internationale \\
\hline $\mathrm{Hg}$. & Herausgeber \\
\hline IZOF & Individual Zones of Optimal Functioning \\
\hline $\mathrm{km} / \mathrm{h}$ & Kilometer pro Stunde \\
\hline KOG & kognitive Zustandsangstkomponente \\
\hline 1 & Liter \\
\hline$\lambda$ & Ljapunov-Exponent \\
\hline $\mathrm{m}$ & Meter \\
\hline Max. & Maximum \\
\hline Min. & Minimum \\
\hline mmol & Millimol \\
\hline nmol & Nanomol \\
\hline RW & Relative Work \\
\hline$\sigma$ & Standardabweichung \\
\hline s & Sekunden \\
\hline S. & Seite \\
\hline SCAT & Sport Competition Anxiety Inventory \\
\hline SOM & somatische Zustandsangstkomponente \\
\hline STAI & State-Trait Anxiety Inventory \\
\hline SW & Selbstwirksamkeitsüberzeugung \\
\hline ü. NN & über Normal Null \\
\hline u.a. & unter anderem \\
\hline u.U. & unter Umständen \\
\hline vgl. & vergleiche \\
\hline zit. & zitiert \\
\hline ZOF & Zone of Optimal Functioning \\
\hline
\end{tabular}




\section{Abstract}

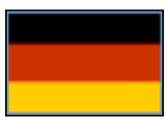

Die vorliegende Dissertation versucht, den Zusammenhang von Angst und sportlicher Leistung beim Freifallformationsspringen mithilfe von Katastrophenoberflächen zu modellieren und ein für das Fallschirmspringen optimales Erregungsniveau zu definieren. In einem theoretischen Teil wird auf Grundlagen eingegangen und eine Einordnung der Anforderungen des Fallschirmspringens vorgenommen. Zentrales Element der Arbeit sind die Untersuchungen an 88 Fallschirmspringern aus 10 Nationen, die im Rahmen einer Weltmeisterschaft und zweier Deutscher Meisterschaften 218 Sprünge unter Trainings- und Wettbewerbsbedingungen durchführten. Die Untersuchungsergebnisse belegen, dass mit dem Programm GEMCAT II angepasste Katastrophenmodelle im Vergleich zu traditionellen Modellen den Angst-Leistungs-Zusammenhang am genauesten beschreiben. Die kognitive Komponente der Zustandsangst sowie die physiologische Aktivierung der Fallschirmspringer werden als entscheidende Größen identifiziert. Zusätzlich wird der Angst-LeistungsZusammenhang durch die Selbstwirksamkeitsüberzeugung, die Eigenschaftsangst und die Sprungerfahrung der Probanden wesentlich beeinflusst. Diese Größen moderieren nicht nur Angst- und Leistungswerte, sondern scheinen in der Lage $\mathrm{zu}$ sein, den Zeitpunkt des Auftretens von Katastropheneffekten zu verschieben.

\section{Abstract}

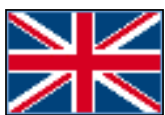

This dissertation deals with modelling the relationship of anxiety and sports performance through catastrophe surfaces and with defining an optimal arousal level for skydiving. In a theoretic part, basic concepts are presented and the demands of skydiving are defined. The central part of the thesis consists of research on 88 parachutists from 10 nations, who made 218 training and competition jumps during one World Championship and two German Formation Skydiving Championships. Research results prove that catastrophe models are best suited to describe the anxiety-performance-relationship when compared to traditional models. Cognitive anxiety as part of the state-anxiety and physiological arousal of the parachutists were identified as important measurements. The anxiety-performance-relationship is strongly moderated by the self-confidence, the trait-anxiety and the jumping experience. Those items seem to act as shifting factors when comparing different catastrophe surfaces fitted with the GEMCAT II program. They also seem to delay the time at which properties predicted by the catastrophe theory occur. 


\section{I $\quad$ Einleitung}

„90\% of sports is mental; the other half is in your head.“ - dieses Zitat von Yogi BERRA (zit. nach TAYLOR 1987, 139) entbehrt zwar einer gewissen Logik, jedoch stellt es recht gut die Haltung vieler Sportler dar: Psychische Faktoren können über Sieg oder Niederlage, über maximale oder minimale Leistung im Sport entscheiden. Einer dieser leistungsbestimmenden Faktoren ist die Angst.

An dieser Stelle sei angemerkt, dass der Angstbegriff im Sprach- und Denkgebrauch unserer Gesellschaft oft negativ besetzt ist. Angst wird durchweg als etwas Schlechtes gesehen. Ziel dieser Arbeit ist es aber, positive Wirkungen von Angst aufzuzeigen, zu demonstrieren, dass ein bestimmtes Angstniveau im Sinne einer größeren Aktivierung - und somit einer besseren Vorbereitung des Körpers auf die bevorstehende sportliche Leistungssituation - für den Sportler von großem Nutzen sein kann. Deshalb wird im Folgenden der Angstbegriff stets wertfrei, mit einer starken Gewichtung der physiologischen Interpretation von Angst, verwendet.

Wenn Angst im Prinzip eine Aktivierung des menschlichen Körpers bewirkt, so ist für bestimmte Angstwerte eine maximale sportliche Leistung zu erwarten. Verlässt ein Sportler diese Zone optimaler Angst oder Erregung, so sollte auch seine Leistung abnehmen. Seit dem Beginn des 20. Jahrhunderts beschäftigen sich Psychologen deshalb mit der Erforschung des Zusammenhangs von Angst und Leistung.

Diese Voraussagen eines anzustrebenden optimalen Angst- bzw. Erregungsniveaus sollen in der vorliegenden Arbeit experimentell überprüft werden, da inzwischen komplexe Modelle zur Verfügung stehen, mit denen sich der Zusammenhang von Angst und sportlicher Leistung erklären lässt. Eine besondere Beachtung gilt hierbei der neuen Katastrophentheorie, die unter anderem plötzliche Leistungsänderungen zu beschreiben vermag. Die vorliegende Arbeit soll dazu erstmals nicht nur ein qualitatives Testen, sondern eine quantitative Analyse des AngstLeistungs-Zusammenhangs mit Hilfe eines Katastrophenmodells ermöglichen.

Die Beschäftigung mit dem Phänomen der Angst und die Frage nach dem Zusammenhang von Angst und sportlicher Leistung begegnete mir allerdings nicht ausschließlich während der sportwissenschaftlichen Forschungstätigkeit. Seit 1991 bin ich aktiver Fallschirmspringer, seit 1994 Mitglied der 8er-Nationalmannschaft im Freifallformationsspringen mit zur Zeit über 5000 absolvierten Fallschirmabsprüngen. Durch diese Kombination von Angsterleben und Angstbewältigung in der Praxis und der Beschäftigung mit zahlreicher Literatur zum Thema 
Angst im (Fallschirm-)Sport innerhalb der Sportpsychologie entstand das Vorhaben, beide Betätigungsfelder miteinander zu verknüpfen.

Diese Arbeit hat zum Ziel, ein für das Fallschirmspringen optimales Angst- bzw. Erregungsniveau innerhalb eines Katastrophenmodells von Angst und sportlicher Leistung zu definieren und experimentell zu überprüfen. Dies bietet die Chance, das Fallschirmspringen aus einem neuen, rein sportwissenschaftlichen, Blickwinkel zu betrachten.

$\mathrm{Zu}$ Beginn dieser Arbeit geht es um die Definition des Angstbegriffes und die Möglichkeiten, Angst zu messen. Neben einer Betrachtung von Angst und Angstbewältigung aus verschiedenen Sichtweisen (Kapitel 1) geht es im einzelnen um die Eigenheiten spezifischer Angstmessinstrumente wie physiologische Messverfahren (Kapitel 2.2.1), Fragebogenerhebungen (Kapitel 2.2.2) und Verhaltensbeobachtungen und der gleichzeitigen Notwendigkeit wie Schwierigkeit einer kombinierten Anwendung dieser Methoden.

Um der grundlegenden Aufgabe dieser Arbeit, der Beschäftigung mit einem optimalen Angstbzw. Erregungsniveau, gerecht zu werden, folgt in Kapitel 3 ein Überblick der bisherigen Theorien zum Zusammenhang von Angst und sportlicher Leistung. Hier findet weiterhin eine Diskussion zusätzlicher Größen, die den Angst-Leistungs-Zusammenhang beeinflussen, statt (Abschnitt 3.2).

An jedem der in diesem Abschnitt vorgestellten Modelle gibt es jedoch Kritikpunkte oder Schwächen im experimentellen Nachweis. Diese Nachteile führen auf die Katastrophentheorie, die aufgrund ihrer Komplexität in Kapitel 4 zunächst erläutert wird, um dann eine praktische Anwendung zur Erklärung des Zusammenhangs von Angst und sportlicher Leistung zu finden.

Da ein optimales Erregungsniveau von sportartspezifischen Elementen abhängen wird, endet der theoretische Teil dieser Arbeit in Kapitel 5 mit einer kurzen Einführung in die Disziplinen und Eigenarten des Fallschirmsports. Eine gesonderte Betrachtung gilt hier den motorischen und kognitiven Anforderungen beim Fallschirmspringen (Abschnitt 5.2.3).

Der anschließende empirische Teil stellt zunächst die Untersuchungsfragestellung, eine Hypothesenbildung, die getestete Probandengruppe und den Verlauf der Untersuchungen dar. Nach einer Beschreibung der Messergebnisse folgt dann der Versuch, an die erhobenen Daten ein Katastrophenmodell von Angst und sportlicher Leistung anzupassen, um Aussagen über das optimale Erregungsniveau für das Fallschirmspringen treffen zu können. 
Daran anschließend wird eine Hypothesenprüfung Aufschluss über den Ausgang der Untersuchungen geben, gefolgt von einer Diskussion über Zustandekommen und Bedeutung der Ergebnisse.

Abgeschlossen wird die Arbeit mit einer kurzen Behandlung der praxisbezogenen Relevanz der Ergebnisse und mit einem Ausblick auf weitere Untersuchungsmöglichkeiten zu diesem Thema. 


\section{Theoretischer Teil}

\section{Angst - ein Begriff aus unterschiedlichen Sichtweisen}

„Angst ist ein unangenehmes und bewusst wahrgenommenes Gefühl, das in Situationen auftritt, die als bedrohlich eingeschätzt werden und die zu einem unangenehmen Erregungszustand führen. " (in: SCHWARZER, 1993, 88).

Zum Begriff der Angst lassen sich eine Vielzahl von Definitionen und Konzepten wie diese in der Literatur finden - auf den ersten Blick scheint es, dass jeder, der sich mit dem Phänomen der Angst beschäftigt hat, eine eigene Definition des Angstbegriffes erstellt hat. Zudem werden die wortverwandten Begriffe Angst, Furcht und Ängstlichkeit gelegentlich synonym gebraucht. Im Folgenden werden daher zunächst einige Definitionen von Angst vorgestellt:

\subsection{Definitionen von Angst}

Angst wird stets als aktueller Zustand verstanden. Angst entsteht, wenn eine Person eine Bedrohung oder einen bedrohlichen Reiz wahrnimmt (vgl. LAZARUS-MAINKA/SIEBENEICK 2000, 11) - bereits FREUD definierte 1926 „Angst ist eine Reaktion auf eine Gefahr“ (FREUD 1926 zit. nach LAZARUS-MAINKA/SIEBENEICK 2000, 12).

HACKFORT und SCHWENKMEZGER geben eine Definition, die den kognitiven Angstaspekt berücksichtigt:

„Angst ist eine kognitive, emotionale und körperliche Reaktion auf eine Gefahrensituation bzw. auf die Erwartung einer Gefahren-oder Bedrohungssituation. Als kognitive Merkmale sind subjektive Bewertungsprozesse und auf die eigene Person bezogene Gedanken anzuführen. (...) Emotionales Merkmal ist die als unangenehm erlebte Erregung, die sich auch in physiologischen Veränderungen manifestieren und mit Verhaltensänderungen einhergehen kann. “

(HACKFORT/SCHWENKMEZGER 1985, 19).

Rost und HAFERKAMP liefern eine Definition, die für die direkte wissenschaftliche Untersuchung oder Überprüfung erste Ansatzpunkte bietet: 
„Angst ist ein Spezialfall eines Erregungs- und Spannungszustandes mit spezifischen somatischen und psychischen Empfindungen und Reaktionen. Sie ist gekennzeichnet durch Vorwegnahme, aktuelle Empfindung oder Erinnerung einer subjektiv bedeutsamen realen oder vorgestellten Unsicherheit (...) bzw. Bedrohung (Versagen, Schmerz, Gefahr) im weitesten Sinne und wird in der Regel durch gelernte Hinweisreize ausgelöst. Angst neigt zur Generalisierung (d.h. Ablösung von den ursprünglich angstauslösenden Ereignissen und Koppelung mit an und für sich unbedrohlichen Reizen) bis hin zur Verselbständigung im Sinne einer generellen Verhaltenstendenz." (Rost/HAFERKAMP 1979, 3 zit. nach SÖRENSEN 1994, 3).

Einen weiteren Definitionsansatz mit Hinweis auf die physiologische Auswirkung des Angstempfindens bietet PSCHYREMBEL:

„Angst ist ein unangenehm empfundener, gleichwohl lebensnotwendiger (weil Gefahr signalisierender) emotionaler Zustand mit zentralem Motiv der Vermeidung bzw. Abwehr einer Gefahr und u.U. psychischen und physischen Begleiterscheinungen: Unsicherheit, Unruhe, Erregung, evtl. Panik, Bewusstseins-, Denk- oder Wahrnehmungsstörungen, Anstieg von Puls- und Atemfrequenz, verstärkte Darm- und Blasentätigkeit, Übelkeit, Zittern, Schweißausbrüche. “

(PSCHYREMBEL 1993, 72).

\subsubsection{Angst und Furcht}

Die Begriffe Angst und Furcht werden in unserer Sprache nahezu beliebig austauschbar gebraucht. Schließlich sind beides Bezeichnungen für eine emotionale Erregung, hervorgerufen durch das Erleben von Gefahr. In der Psychologie und der Angsttheorie wird dagegen eine Unterscheidung von Angst und Furcht anhand des Auslösers vorgenommen: Angst vor einer bestimmten, genau definierten Gefahr wird als Furcht bezeichnet.

Der Angst als solcher ist kein spezielles Gefahrenmoment als Auslöser zugeordnet, sie ist also quasi ungerichtet (vgl. LAZARUS-MAINKA/SIEBENEICK 2000, 14). 
Angst ist also die Reaktion auf eine mehrdeutige Gefahrensituation, in der eine angemessene Handlung nicht möglich scheint - Furcht dagegen auf eine eindeutige Gefahrenquelle gerichtet (vgl. SÖRENSEN 1994, 4). Siehe hierzu auch die folgende Tabelle 1.

Tabelle 1: Merkmale von Angst und Furcht (vgl. SÖRENSEN 1994, 4).

\begin{tabular}{|l|l|}
\hline Angstmerkmale & Furchtmerkmale \\
\hline Gefahrenreiz & Eindeutige Gefahrenquelle \\
\hline Reaktionsblockierung & Fluchttendenz \\
\hline $\begin{array}{l}\text { Unsicherheits- bzw. } \\
\text { Mehrdeutigkeitserlebnis }\end{array}$ & \\
\hline
\end{tabular}

\subsubsection{Angst als Zustand und als Eigenschaft}

Im täglichen Leben begegnet man unterschiedlichen „Angsttypen“ - Menschen, die deutlich „mutiger“ oder aber „ängstlicher“ als andere erscheinen. Somit scheint auch eine Differenzierung zwischen der momentanen Angst und der Angst, die vom Angsttyp her resultiert, sinnvoll. SPIELBERGER (1972) führte diese Unterscheidung in seiner State-Trait-AnxietyTheorie ein und differenzierte zwischen Zustandsangst (State-Anxiety) und Eigenschaftsangst (Trait-Anxiety) (vgl. LAZARUs-MAINKA/SIEBENEICK 2000, 16).

Diese Eigenschaftsangst wird auch Ängstlichkeit genannt und beschreibt, wie stark eine Person auf einen Reiz hin mit Angst reagiert (vgl. HACKFORT/SPIELBERGER 1989, 5). SPIELBERGER definierte Ängstlichkeit als „,... individuelle Unterschiede in der Tendenz, die Welt als gefährlich und bedrohlich anzusehen, und in der Häufigkeit, mit der Angstzustände über längere Zeiträume hinweg erlebt werden“ (SPIELBERGER 1985, 176 zit. nach LAUX/ GLANZMANN 1996, 122).

Je höher die Ängstlichkeit einer Person ist, desto größer ist die Angst, mit der sie auf eine Stress-Situation reagieren wird.

Eine Einschränkung dieses Zusammenhangs gilt allerdings bei Stress-Situationen, die durch eine physische Gefährdung entstehen: Hier scheint die Intensität der Angstreaktion unabhängig von der jeweiligen Ängstlichkeit ${ }^{1}$ zu sein (vgl. LAUX/GLANZMANN 1996, 117 sowie LAUX et al. 1981, 8).

\footnotetext{
${ }^{1}$ Gemessen mit der Trait-Angstskala des STAI-Fragebogens.
} 
Statistisch betrachtet wird jedoch der Mensch mit deutlich mehr Situationen konfrontiert, die den Selbstwert in Frage stellen ${ }^{2}$, als mit physischen Gefährdungssituationen. Damit ist die Aussage, dass Hochängstliche dazu tendieren, mehr Situationen als bedrohlich einzustufen und auf sie mit einem höheren Zustandsangstanstieg zu reagieren, noch immer haltbar (vgl. LAUX et al. 1981, 8).

Dieses Argument wird im konkreten Anwendungsfall der Sportpsychologie nochmals bestätigt: Untersuchungen zeigten, dass die Wettkampfangst im Leistungssport eine nahezu ideale Realisierung von selbstwertrelevanten Stress-Situationen ist (vgl. GLANZMANN 1985, 166; LAZARUS 2000, 244).

Zusammengefasst ist Zustandsangst (State-Anxiety) also die Angstreaktion auf einen Gefahrenreiz. Sie ist akut, von starker Intensität und zeitlich schnell vorübergehend.

Demgegenüber ist Eigenschaftsangst oder Ängstlichkeit von geringerer Intensität, dafür aber von unbestimmter Dauer. Die Ängstlichkeit stellt ein Persönlichkeitsmerkmal dar (vgl. SöRENSEN 1994, 6). Die Begriffe Eigenschaftsangst und Ängstlichkeit werden im Folgenden synonym verwendet.

\subsubsection{Angst und Erregung}

Im Rahmen der Arousal-Theorie wird Angst als eine spezifizierte physiologische Erregung betrachtet. Diese Erregung soll dazu dienen, den Körper auf mögliche Reaktionen für die Gefahrensituation bzw. den externen Reiz vorzubereiten oder zu aktivieren ${ }^{3}$. Statt des Begriffes der Erregung findet man vielerorts auch den Ausdruck Aktivierung/Aktivaktion (engl. ,activation“) (vgl. HACKFORT/SCHWENKMEZGER 1985, 24). Man versucht also, eine Angstskala mit den Endwerten der Angstfreiheit (Ruhe) und der hohen Angst (Erregung) mit einer Aktivierungsdimension mit den Polen Schlaf und Wachheit in Verbindung zu bringen. Allerdings besteht hier der wichtige Unterschied, dass hohe Werte auf der Angstebene als negativ, hohe Aktivierungs- oder Wachheitszustände dagegen als positiv empfunden werden (vgl. LAUX/GLANZMANN 1996, 131).

\footnotetext{
${ }^{2}$ Angstsituationen wie Prüfungs- oder Wettbewerbsangst, Selbstzweifel.

${ }^{3}$ Mögliche Reaktionen sind dabei Flucht oder - aus der Tierwelt bekannt - Angriff.
} 


\subsubsection{Somatische und kognitive Angst}

Für die heutige Angstforschung ist eine weitere Differenzierung der Zustandsangst vonnöten. Die mehrdimensionale Angsttheorie von MARTENS (1990) unterscheidet zwischen der somatischen und der kognitiven Angst- bzw. Erregungskomponente. Die somatische Angst (auch emotionale Erregung genannt) stellt hierbei das Maß der „Aufgeregtheit“ und (physiologischen) Erregung dar. Sie bezieht sich auf die subjektive Wahrnehmung und Einschätzung von Körpersymptomen des Angsterlebens.

Die kognitive Angstkomponente oder Besorgtheit beschreibt den kognitiven Aspekt des Angsterlebens ${ }^{4}$. Diese beiden Komponenten sind zwar nicht unabhängig voneinander - sie wirken sich allerdings unterschiedlich auf das Verhalten aus (vgl. LAUX/GLANZMANN 1996, 129).

Vor allem im zeitlichen Verlauf zeigen sich deutliche Unterschiede von kognitiver und somatischer Angstkomponente. Untersuchungen von MARTENS (1990) und PARFITT/HARDY (1987) zeigten, dass Sportler während einer Vor-Wettkampfphase bis zu 48 Stunden vor Wettkampfbeginn bereits Maximalwerte der kognitiven Angstkomponente aufwiesen, während die somatische Angstkomponente erst frühestens 6 Stunden vor Wettkampfbeginn ansteigt und ihr Maximum erst im Wettkampf erreicht (vgl. MCNALly 2002; MARTENS et al. 1990, 74).

Im Rahmen einer Faktorenanalyse bei der Konstruktion des Sportangst-Fragebogens CSAI- $2^{5}$ entdeckte MARTENS, dass sich die kognitive Angstkomponente der Besorgtheit in zwei Komponenten aufteilt ${ }^{6}$. Dies ist zum einen die Komponente der negativen Gedanken oder Sorgen und zum anderen eine Komponente von positiven (selbstüberzeugten) Gedanken. Diese positive Komponente, die sogenannte Selbstwirksamkeitsüberzeugung ${ }^{7}$ als Maß der Selbstsicherheit, wurde so zu einer eigenen Messgröße (vgl. PARFITT et al. 2000, 47; MARTENS et al. 1990, 24) - vor allem im Zusammenhang mit sportlicher Bewegung schreibt man der Selbstwirksamkeit eine besondere Rolle zu (vgl. LAZARUs 2000, 237).

\footnotetext{
${ }^{4}$ Im Sinne von sich Sorgen machen, möglichst gut abzuschneiden, negative Eindrücke bei anderen zu vermeiden und sich keine Blöße zu geben (vgl. LAUX/GLANZMANN 1996, 129).

${ }^{5}$ Competitive State Anxiety Inventory-2.

${ }^{6}$ Im Grunde genommen war dies allerdings keine Neuentdeckung, sondern eine Bestätigung früherer Ergebnisse von MACKAY et al. (1978 zit. nach PARFITT et al. 2000, 47).

${ }^{7}$ Im Folgenden wird anstelle von Selbstwirksamkeitsüberzeugung häufig der abgekürzte Begriff der Selbstwirksamkeit verwendet.
} 


\subsection{Physiologische Betrachtung von Angst}

\subsubsection{Neurale Grundlagen von Emotionen}

Emotionale Zustände des Menschen - wie „Angst“, „Furcht“, „Freude“ sind Reaktionen auf äußere Reize. Diese Reiz-Reaktionsverarbeitung und -bewertung wird der Amygdala, einem Teil des lymbischen Systems, zugeschrieben. Die Verarbeitung emotionaler Kategorien wie Ärger und Wut, Neugierde und Freude, Sorge und Angst sowie Trauer und Panik findet hier statt (vgl. Roth/Menzel 1996, 477). Die Amygdala erhält die zu bewertenden Reize von Gebieten der sensorischen Informationsverarbeitung im Kortex und im Thalamus. Die Verarbeitung dieser Reize findet dann unbewusst statt. Als „Ausgang“ der verarbeiteten Reaktion ist die Amygdala umfangreich direkt und indirekt mit motorischen Systemen im Gehirnstamm verbunden, eine indirekte Verschaltung mit dem Neokortex ermöglicht eine emotionale Reizverarbeitung (Aufmerksamkeit, Gedächtnisbildung, Logik).

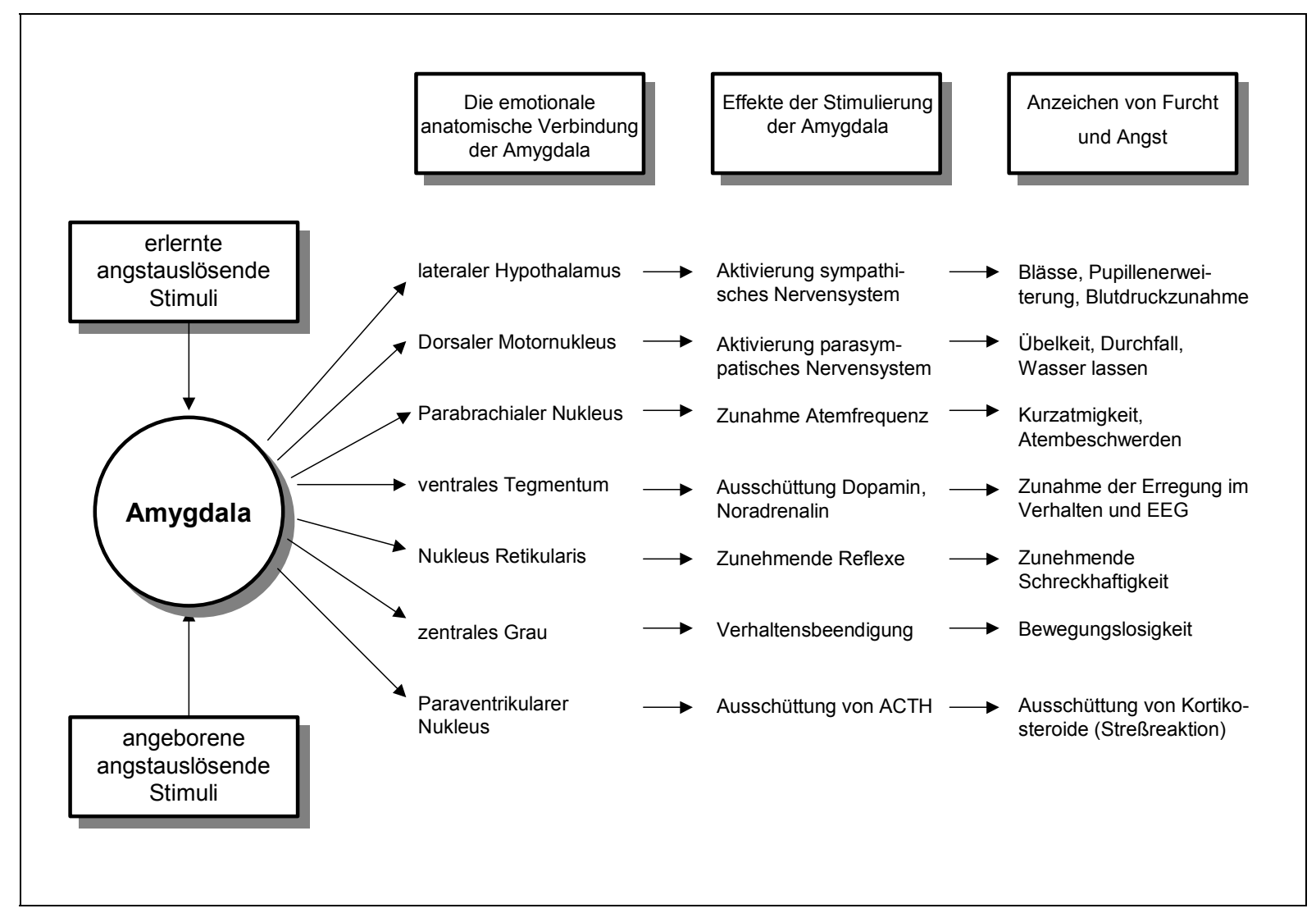

Abbildung 1: Reaktionsauslösung durch die Amygdala (vgl. ROTH/MENZEL 1996, 478). 
Jede Angstreaktion in diesem Netzwerk kann man sich nun als „Verschaltung“ von jeweiligen Eingangsgrößen ${ }^{8}$ oder Eindrücken einer Situation mit der entsprechenden Reaktion vorstellen. Dabei sind zunächst einige Reaktionen oder Verschaltungen angeboren - später werden diese Verschaltungen im Netzwerk des lymbischen Systems durch Erlernen und Erleben weiterer angstauslösender Situationen ergänzt und erweitert. Gerade bei erwachsenen Menschen werden die meisten Emotionen durch eben diese zusätzlich erlernten Stimuli ausgelöst (vgl. ROTH/MENZEL 1996, 478f).

Emotionale Zustände wie z.B. Angst lassen sich so als Zustände höherer Aktivierung eines Gehirngebietes (etwa durch EEG-Aufzeichnungen) nachweisen.

Psychologen können nun Aussagen über den Grad der Aktivierung machen, um emotionale Zustände zu beschreiben oder zu vergleichen. Dieser Grad der Aktivierung beschreibt dabei, wie reaktionsbereit der Organismus ist. Der untere Punkt der Aktivierungsskala wird hier z.B. von Schlaf, das obere Ende durch Zustände rasender Erregung gekennzeichnet (vgl. BERLYNE 1974, 73).

Der Mensch reagiert also auf Situationen des Drucks oder besonderer Herausforderungen mit einer nach außen sichtbaren physiologischen Reaktion, die ihn auf mögliche Aktionen vorbereitet. Es soll so mehr Kraft, Aktionspotential etc. bereitgestellt werden können. „When a person feels strong emotion, such as fright, anger, or excitement, the body prepares itself for action." (in: CLARKSON 1999, 6). Diese physiologische Aktivierung stellt im Prinzip seinerseits eine Reaktion auf die Reizung der Amygdala dar.

\subsubsection{Physiologische Aktivierung}

Die physiologische Aktivierung, also „das Ende“ der Reiz-Reaktionskette ${ }^{9}$ zeigt viele im Folgenden beschriebene Aktivierungsgrößen bei Angst und Furcht. Als offensichtlichstes Merkmal beobachtet man eine Erhöhung von Herz- und Atemfrequenz sowie eine Erhöhung des Blutdruckes. Gleichzeitig ist eine Pupillenerweiterung, eine Zunahme des Hautwiderstands und eine vermehrte Schweißsekretion registrierbar. Es kommt zu einem Erblassen und einer Steigerung der Reflexe - somit auch zu einer erhöhten Schreckhaftigkeit (vgl. LAZARUSMAINKA/SiEBENEICK 2000, 60f; VALLERAND/BLANCHARD 2000, 5).

\footnotetext{
${ }^{8}$ Sensorische Reize, Gerüche, optische und akustische Reize, Geschmäcke, sensorische Rückmeldungen (vgl. Roth/MENZEL 1996, 477).

${ }^{9}$ Oder die direkt von außen objektiv beobachtbare Wirkung.
} 
Da das Nervensystem auf Glucose als Energielieferant angewiesen ist, beobachtet man biochemisch eine Zuckerfreigabe ins Blut, wiederum um Energiereserven für eine mögliche Reaktion auf den Angstreiz bereitzustellen - gleiches gilt für den messbaren Anteil freier Fettsäuren im Blut (vgl. NORUM/URSIN 1978, 76; EIDE/ATTERÅs 1978, 99).

Weiterhin ist im Blut eine Ausschüttung von Hormonen festzustellen. Diese Hormone sind im Einzelnen:

- Dopamin (stimuliert Aggression und Aufmerksamkeit, verbessert Koordination)

- Adrenalin (erhöht Herz-, Atemfrequenz, Muskelanspannung und Blutdruck, Fluchtreaktion von ,fight or flight")

- Noradrenalin (erhöht Herzfrequenz und Muskelanspannung, verbessert Alarmiertheit und Reaktionsschnelligkeit, Kampfreaktion von „,fight or flight“)

- Kortisol (erhöht Blutzuckergehalt und Adrenalinempfindlichkeit) (vgl. CLARKSON 1999, 7-9)

In der folgenden Tabelle findet sich eine Auflistung der unterschiedlichen metabolischen Produkt- und Hormonkonzentrationen im Blut zu verschiedenen Zeiten bei Fallschirmspringern aus einer Untersuchung von ANFILOGOFF et al. (1987).

Tabelle 2: Mittlere Hormon- und Stoffwechselproduktkonzentration im Blut (Standardabweichung in Klammern ${ }^{10}$ ) zu verschiedenen Zeiten beim Fallschirmspringen (in: ANFILOGOFF et al. 1987, 415).

\begin{tabular}{|c|c|c|c|c|c|}
\hline Hormon & Grundwert & $\begin{array}{c}\text { Besteigen } \\
\text { des } \\
\text { Flugzeugs }\end{array}$ & $\begin{array}{c}\text { Unmittelbar } \\
\text { vor dem } \\
\text { Absprung }\end{array}$ & $\begin{array}{c}\text { Unmittelbar } \\
\text { nach der } \\
\text { Landung }\end{array}$ & $\begin{array}{c}\text { 20 Minuten } \\
\text { nach der } \\
\text { Landung }\end{array}$ \\
\hline $\begin{array}{c}\text { Adrenalin } \\
\text { (nmol/1) }\end{array}$ & $0,50(26)$ & $0,82(37)$ & $2,21(106)$ & $2,11(111)$ & $0,56(20)$ \\
\hline $\begin{array}{c}\text { Noradrenalin } \\
\text { (nmol/1) }\end{array}$ & $1,76(58)$ & $2,66(55)$ & $4,24(123)$ & $6,35(193)$ & $4,46(133)$ \\
\hline $\begin{array}{c}\text { Kortisol } \\
\text { (nmol/1) }\end{array}$ & $359(120)$ & $221(197)$ & $466(289)$ & $561(333)$ & $685(334)$ \\
\hline $\begin{array}{c}\text { Glucose } \\
\text { (mmol/l) }\end{array}$ & $4,9(3)$ & $4,6(5)$ & $5,3(3)$ & $5,8(5)$ & $5,7(4)$ \\
\hline $\begin{array}{c}\text { Laktat } \\
(\mathrm{mmol} / \mathrm{l})\end{array}$ & $0,84(12)$ & $1,13(51)$ & $1,47(36)$ & $4,48(39)$ & $1,66(70)$ \\
\hline
\end{tabular}

\footnotetext{
${ }^{10}$ Die Standardabweichung $\sigma$ wird im Folgenden stets wie in den Naturwissenschaften üblich angegeben: Die Werte in Klammern beziehen sich stets auf die letzten Stellen des vorherigen Wertes und haben gleich viele Nachkommastellen. 0,50(26) bedeutet also den Wert 0,50 mit einem $\sigma$ von 0,26-2,21(106) den Wert 2,21 mit einem $\sigma$ von 1,06 .
} 
Man erkennt gegenüber dem Ruhewert eine Vervierfachung der Adrenalinkonzentration, der Noradrenalinwert steigt um das Dreieinhalbfache, die Kortisolkonzentration verdoppelt sich nahezu. Allerdings werden die jeweiligen Maximalwerte zu unterschiedlichen Zeitpunkten erreicht (vgl. ANFILOGOFF et al. 1987, 415).

Vorangegangene Untersuchungen von URSIN et al. (1978) und RICHTER et al. (1996) erhielten identische Tendenzen im Verlauf der Kortisol- und Glucose-Konzentrationen im Blut von norwegischen und deutschen Erstspringern. Die restlichen in obiger Tabelle aufgeführten Hormone wurden in dieser Untersuchung nicht erfasst (vgl. LEVINE 1978, 53f; EIDE/ATTERÅs 1978, 101f; RICHTER et al. 1996, 1957f).

Ferner wird im Zentralnervensystem die Aktivität der Alphawellen, die sonst bei einem entspannten Zustand vorkommt, unterbrochen. „Unruhige“ Betawellen nehmen zu und signalisieren Konzentration sowie einen angespannten Zustand (vgl. SöRENSEN 1994, 107).

Viele dieser Reaktionen basieren auf Funktionen des Sympathikus, dem sogenannten Leistungsnerv, der z.B. für Umstellungen des Organismus zur Leistungsverbesserung verantwortlich ist. Diese Leistungsverbesserungen mit oben genannten Faktoren dienen der optimalen Aktivierung und Reaktionsfähigkeit des Körpers auf äußere Reize. Wesentliches Überträgerhormon des Sympathikus ist hierbei das Noradrenalin, das bei körperlicher Belastung oder auch Angstreaktionen u.a. ein Ansteigen der Herzfrequenz bewirkt (vgl. Hollmann/Hettinger 2000, 83; Rost 2001a, 383). Die Noradrenalinwerte in Tabelle 2 verdienen somit eine besondere Beachtung.

Ist hier allerdings von optimaler Aktivierung und Leistungsverbesserung die Rede, kommt schnell die Vermutung auf, dass eine solche Aktivierung auch zur Verbesserung einer sportlichen Leistung genutzt werden könnte (vgl. STEINBACH 1968, 203; BIEDERMANN 2002, 103f). So wäre eine Verbesserung der Leistung durch eine „richtige“ und optimale Aktivierung mit Emotionen wie Freude, Angst oder Furcht anzustreben. Das „Nutzen“ von Emotionen bzw. das Erforschen des tatsächlichen Zusammenhangs zwischen Angst, Aktivierung und Leistung fällt so schnell wieder in den Forschungsbereich der Sportpsychologie. 


\subsection{Bewältigung von Angst - Coping}

Betrachtet man die Dynamik der beobachteten Angstreaktionen in einer angstauslösenden Situation, so stellt man fest, dass die Angstreaktion einen Maximalwert etwa zu Beginn des Angstereignisses aufweist. Nach diesem Zeitpunkt nimmt die Angststärke nicht weiter zu, um die Aktivierung des Körpers zur Reaktion auf die äußere Situation nicht zu beeinträchtigen (vgl. Rost 2001b, 339f; LEVINE et al. 1978, 6).

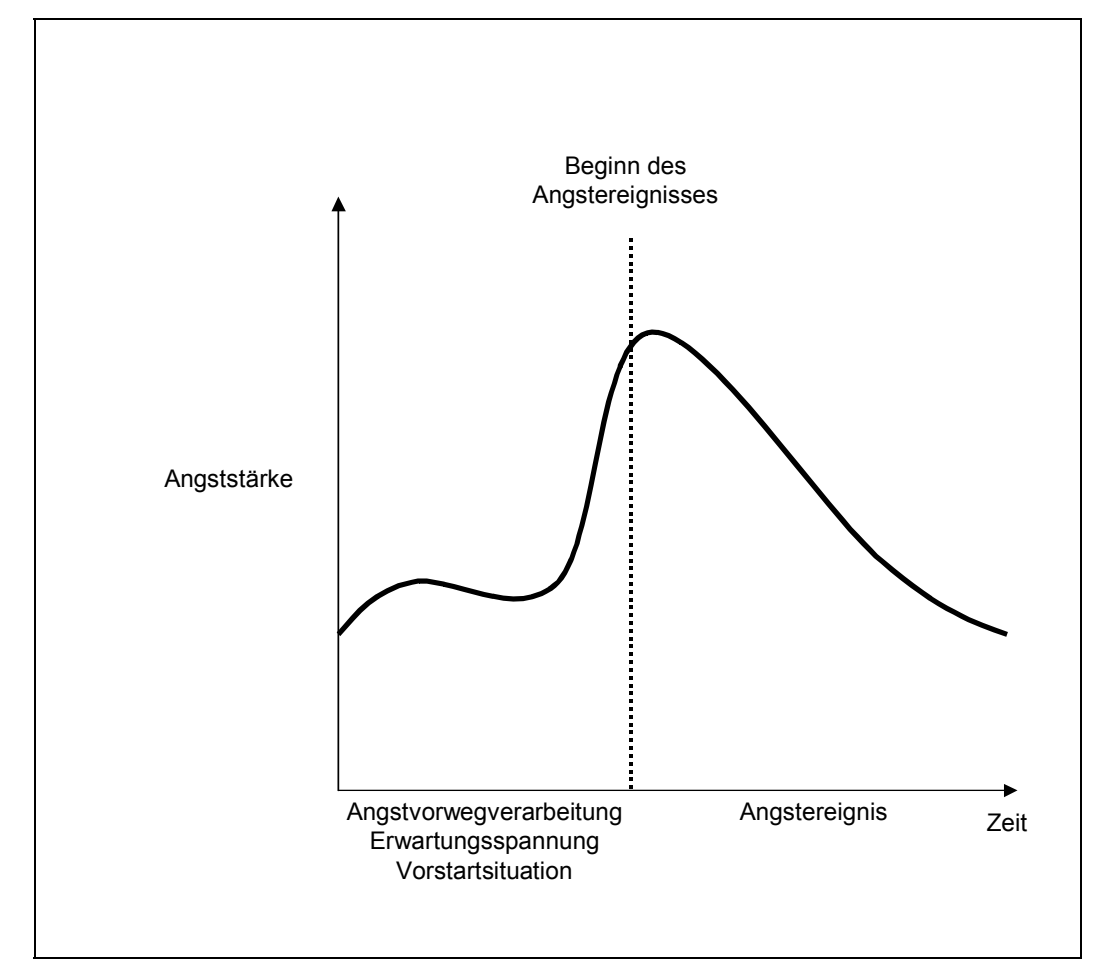

Abbildung 2: Angstdynamik (vgl. RoST 2001b, 340).

Dieser zeitliche Verlauf der Angststärke ist nur zu erreichen, wenn direkt nach der Bewertung der angstauslösenden Situation auch bereits Angstbewältigung einsetzt ${ }^{11}$. Diese Angstbewältigung (engl. „Coping“) setzt direkt nach Bewertung einer Situation als bedrohlich ein (vgl. SÖRENSEN 1994, 28). Unter Coping ${ }^{12}$ versteht man also den gesamten Vorgang der Änderung von Verhalten, kognitiven Prozessen oder physiologischen Faktoren (vgl. LEVINE et al. 1978, 6f).

\footnotetext{
${ }^{11}$ Da der Angstreiz in der angstauslösenden Situation auch während des Angstereignisses vorhanden ist, würde die Angststärke sonst während des Angstereignisses weiter ansteigen.

${ }^{12}$ Im Folgenden werden die Begriffe Angstbewältigung und Coping synonym verwendet.
} 
„Coping may be defined as a process of using cognitive and behavioral efforts to manage specific external and/or internal demands that are appraised as taxing or exceeding the resources of the person." (in: LAZARUS/FOLKMAN 1984, 141).

Dabei kann dieser Vorgang sowohl präemotional, das Angsterleben begleitend oder postemotional ablaufen (vgl. LAUX/GLANZMANN 1996, 134).

Dies bedeutet aber, dass man bei jeder Messung und Betrachtung von Angst stets ein durch bereits erfolgtes bzw. parallel ablaufendes Coping „gefilterte“ Werte erhält - die Angstbewältigung stellt einen konstant vorhandenen, situationsabhängigen Moderator der Angstintensität dar.

Von Bedeutung sind allerdings unterschiedliche Vorgehensweisen, Strategien und Stile des Copings. Das Wissen um die Wahl einer für die spezielle Situation geeigneten Art der Angstbewältigung kann so im Sport nützlich sein, um erwünschte Erregungszustände zu erreichen.

\subsubsection{Coping-Strategien}

Betrachtet man den Prozess des Copings, so lässt sich das Angstbewältigen eines Probanden anhand der konkreten beobachtbaren Reaktionen nach Coping-Arten bzw. Coping-Strategien differenzieren. Nach LAZARUS unterscheidet man dabei zunächst zwischen instrumentellen (engl. ,problem-focused-coping“) und palliativen (engl. „emotion-focused-coping“) Angstbewältigungsformen (vgl. SöRENSEN 1994, 28; EUBANK/COLLINS 2000, 122).

\subsubsection{Instrumentelle Angstbewältigung}

Die instrumentelle Bewältigung wirkt auf das Verhältnis zwischen Person und Umwelt ein mit dem Ziel der Änderung der Situation. Es werden kognitive oder verhaltensbeobachtbare Versuche einer Problemidentifizierung und -lösung unternommen. Beispiele für solche Coping-Strategien sind z.B. die Neudefinition von Grenzen, Regeln, Vorgaben oder Zielen, die Identifikation alternativer Quellen der Befriedigung in der Situation, die Suche nach sozialer Unterstützung, das Aneignen neuer Fähigkeiten und Fertigkeiten, die Erhöhung eigener Anstrengung oder die Inanspruchnahme externer Hilfen durch Trainer, Experten oder Psychologen (vgl. Anshel et al. 2000, 753; GiacobBi/WeinBerg 2000, 43). 


\subsubsection{Palliative Angstbewältigung}

Die palliative Bewältigungsform dient einer Regulierung der Emotion, ohne auf die Angstsituation selbst einzuwirken. Gleichzeitig wird mitunter die Interpretation des angstauslösenden Stimulus verändert, sodass die Bedeutung des Stressors für die Person verändert wird. Beispiele für diese Coping-Arten sind die Leugnung oder Verharmlosung einer Gefahrensituation oder der Rückzug in Wunschdenken oder Humor. Palliative Bewältigungsformen werden hauptsächlich in nicht-kontrollierbaren Situationen eingesetzt (vgl. SÖRENSEN 1994, 29; ANSHEL et al. 2000, 753; GiACOBBi/WEINBERG 2000, 43).

\subsubsection{Coping-Stile}

In der kognitiven Bewertung einer angstauslösenden Situation neigen Personen (ausgehend von individuellen Merkmalen, Erfahrungen oder Präferenzen) dazu, aus den zur Verfügung stehenden Coping-Strategien nur bestimmte bevorzugt auszuwählen. Diese Wahl aus den oben angeführten Bewältigungsstrategien wird als Coping-Stil bezeichnet. Man differenziert dabei die Coping-Stile nach zwei auftretenden Klassen:

- Anstreben von Kontrolle über die Bedrohung (engl. ,approach coping“"), auch als Vigilanz bezeichnet (vgl. HINDEL/KROHNE 1988, 42)

- Meidung bedrohensbezogener Informationen, Vermeidung (engl. ,repressive coping“)

Innerhalb dieser Klassen sind sowohl instrumentelle als auch palliative Bewältigungsformen zu finden - Coping-Stile entstehen nur aus den Vorzügen eines Individuums bei der „typischen“ Auswahl von Coping-Strategien (vgl. ANSHEL et al. 1997, 142f; ANSHEL et al. 2000, 754f; HiNDEL/KROHNE 1988, 42).

\subsubsection{Coping in Abhängigkeit von Persönlichkeits- und Situationsmerkmalen}

Ausgehend von einer differentialpsychologischen Betrachtungsweise stellt sich die Frage, welche weiteren Faktoren über die Wahl konkreter Angstbewältigungs- oder CopingStrategien entscheiden. Neben dem Coping-Stil scheinen hierbei die persönlichen Variablen der Ängstlichkeit (Trait-Anxiety), der Erfahrung ${ }^{13}$ und des Geschlechts von Bedeutung zu

\footnotetext{
${ }^{13}$ Der Begriff der Erfahrung versteht sich hier als „Angsterfahrung“, also das Wissen über erfolgreiche Angstbewältigung in vergleichbaren Situationen (vgl. URSIN 1978, 217; LAZARUS 2000, 233).
} 
sein. Weiterhin spielt das Erleben der konkreten Situation und eine Evaluation der eigenen Ziele und Handlungsmöglichkeiten eine entscheidende Rolle bei der Wahl einer „passenden“ Coping-Strategie (vgl. HindEL/KROHNE 1988, 42; ANSHEL et al. 1997, 147).

Hinsichtlich der Ängstlichkeit zeigt u.a. eine Untersuchung von GIACOBBI und WEINBERG (2000), dass mit steigenden Werten der Eigenschaftsangst von den Probanden zunehmend palliative Angstbewältigungsstrategien wie Leugnung, Wunschdenken und Humor in (sportlichen) Angstsituationen angewandt werden (vgl. GiACOBBi/WeInBERG 2000, 58f). In gewissen sportlichen Situationen sind diese repressiven palliativen Strategien aber für das Erreichen optimaler Leistung eher hinderlich: Ein Sportler, der Frustration oder Angst erlebt, sollte eher aktive, instrumentelle Bewältigungsformen ${ }^{14}$ anstreben. Statistisch zeigt sich, dass diese instrumentellen Coping-Strategien zumindest von Mannschaftssportlern bevorzugt gegenüber palliativen oder Vermeidungsstrategien genutzt werden (vgl. NTOUMANIS/BIDDLE 2000, 362; GIACOBBI/WEINBERG 2000, 59).

Andererseits zeigten Versuche von Hindel/KroHne (1988) und ANSHEL et al. (2000) an Individualsportlern umgekehrte Tendenzen. Diese Sportler wendeten verstärkt Vermeidungsstrategien an, um eine maximale Leistung zu erreichen. Dieser Unterschied wird von den Autoren dadurch erklärt, dass in den Individualsportarten mitunter repressive Strategien als „Ausblendung“ unerwünschter, ablenkender Sinneseindrücke oder Emotionen durchaus von Nutzen sein können, um z.B. bessere Konzentrationsleistungen im Rahmen der zu bewältigenden motorischen Aufgabe zu ermöglichen (vgl. HindEL/KrOHNE 1988, 49; ANSHEL et al. 2000, 769). Mannschaftssportarten hingegen erfordern häufig eine viel zu breit gefächerte Aufmerksamkeit unterschiedlichster Komponenten, sodass hier eine repressive „Ausblendung“ nicht sinnvoll erscheint (vgl. D’URSO et al. 2002, 193).

Betrachtet man die Erfahrung von Probanden innerhalb einer Angstsituation, so lassen sich sehr deutliche Vorteile von „Experten“ gegenüber Anfängern in einer identischen Angstsituation nachweisen. So zeigten beispielsweise die Untersuchungen an Fallschirmsprungschülern von URSIN et al. (1978), dass bei Fallschirmspringern bereits nach etwa einer Woche Sprungdienst die physiologischen Messgrößen der Konzentrationen von Kortisol und freien Fettsäuren im Blut der Probanden wieder ihren Grundwert erreicht hatten (vgl. LEVINE 1978, 54f). Fragebogenmessungen zum kognitiven Erleben der Angst beim Fallschirm-

\footnotetext{
${ }^{14}$ Wie z.B. eine größere Anstrengung oder die kognitive Entwicklung von Lösungsplänen.
} 
springen bestätigten, dass die fortgeschrittenen Springer deutlich niedrigere Angstwerte aufgrund neu gebildeter Coping-Strategien aufwiesen. Identische Resultate mit einer starken Gewichtung dieser kognitiven Effekte wurden von KARGE (1994) und FALK/BAR-ELI (1995), ebenfalls in Untersuchungen an Fallschirmsprungschülern und erfahreneren Springern, erzielt (vgl. KARGE 1994, 78f) - gleiches gilt für die Untersuchung von DuRTsCHI (2001) (vgl. DURTSCHI 2001, 192f). Aufgrund einer geringen Probandenzahl konnte die statistische Signifikanz in der Untersuchung von FALK/BAR-ELI allerdings nicht für alle Teilergebnisse nachgewiesen werden (vgl. FALK/BAR-ELI 1995, 116).

Bezüglich des Geschlechts wird in der Literatur von Unterschieden bei der typischen Wahl von Bewältigungsstrategien berichtet. So sollen Frauen häufiger als Männer auf palliative Coping-Strategien im Sport zurückgreifen (vgl. ANSHEL et al. 1997, 145).

Zusammenfassend sei nochmals hervorgehoben, dass Angstbewältigung aufgrund der zeitlich parallelen Auslösung und der stetigen Rückkopplung während des Angsterlebens als integraler Bestandteil der Emotion Angst anzusehen ist. Jede Untersuchung oder Messung von Angst betrachtet eine stetige dynamische Wechselwirkung von Angstreiz, Bewertung und Bewältigung (vgl. LAZARUs 2000, 235).

Das Wissen um die verschiedenen Coping-Strategien und -Stile ist für den einzelnen Sportler im Sinne einer optimalen Emotionsregulation wichtig. Durch die Auswahl einer für die jeweilige Situation „passenden“ Angstbewältigungsstrategie lassen sich erwünschte Erregungszustände gezielt ansteuern - weiterhin kann das naive Wählen unzweckmäßiger Coping-Arten mit daraus möglicherweise resultierenden Leistungseinbußen vermieden werden (vgl. NTOUMANis/BidDLE 2000, 369; ANSHEL et al. 2000, 771). 


\section{Angstmessung}

Um eine objektive Beschreibung von Angst und z.B. den Vergleich von Angstzuständen zu ermöglichen, benötigt man Instrumente zum Messen von Angst. Es können aber natürlich immer nur die Auswirkungen von Angst, die Reaktionen auf das Angsterleben, gemessen werden - Angst als Konstrukt ist nicht direkt messbar. Man benötigt also diagnostische Verfahren, die nach außen sichtbare Indikatoren von Angst messbar machen. Diese Indikatoren des Angsterlebens lassen sich nach allgemeinen Komponenten der Angst unterscheiden (vgl. SÖRENSEN 1994, 106; LAZARUS-MAINKA/SIEBENEICK 2000, 37f).

\subsection{Angst-Indikatoren}

Nach BECKER (BECKER 1980, 20f zit. nach SÖRENSEN 1994, 106) lassen sich folgende vier Komponenten der Angst als Indikatoren des Angsterlebens wiederfinden:

1. Kognitive Indikatoren

2. Affektive Indikatoren

3. Physiologische Indikatoren

4. Motorische Indikatoren

\subsubsection{Kognitive Indikatoren}

Auf der kognitiven Ebene findet eine Bewertung, eine gedankliche Auseinandersetzung mit einer subjektiv als gefährlich gedeuteten Situation statt. Die Situation wird vor dem Hintergrund von zuvor Gelerntem analysiert, Handlungsmöglichkeiten werden abgewogen. Eine Angstmessung kann hier entweder situationsunabhängig ${ }^{15}$ oder aber unter Berücksichtigung der speziellen Angstbegebenheit stattfinden - eine mögliche konkrete Angstbegebenheit wäre hier z.B. die Sportangst (vgl. HACKFORT/SCHWENKMEZGER 1989, $56 f)$.

\footnotetext{
${ }^{15}$ Also bezüglich der Trait-Anxiety-Komponente (der Eigenschaftsangst).
} 


\subsubsection{Affektive Indikatoren}

Die affektiven Angstindikatoren beschreiben den unangenehmen Gefühlszustand der Angst, also gewissermaßen die somatische Angstkomponente. Dieser Angstzustand wird durch Begriffe wie Hilflosigkeit, Unsicherheit oder Unruhe gekennzeichnet. Allerdings lässt sich auch ein erhöhtes Erregungs- oder Aktivierungsniveau (z.B. im Sinne einer inneren Spannung) diesem Indikator zuordnen (vgl. SöRENSEN 1994, 107).

\subsubsection{Physiologische Indikatoren}

Als physiologische Angstindikatoren werden die vielfältigen Veränderungen des Organismus mit dem Ziel, auf gefährliche Reaktionen mit Flucht oder Angriff reagieren zu können, gewertet. Diese Indikatoren wurden bereits im Abschnitt 1.2 auf Seite 9 aufgeführt und lassen sich in respiratorische und kardiovaskuläre Indikatoren, sowie in biochemische und elektrophysiologische ${ }^{16}$ Indikatoren aufteilen (vgl. HACKFORT/SCHWENKMEZGER 1989, 58).

\subsubsection{Motorische Indikatoren}

Motorisch wird ein Angstzustand z.B. durch einen hohen Spannungszustand der Muskulatur charakterisiert, der mitunter bis zu Verkrampfungen führen kann. Weiterhin sind motorische Unruhe, Händezittern, Mimik und auch Sprachstörungen verschiedener Art ein Indikator für Angst (vgl. SöRENSEN 1994, 108).

\subsection{Messmethoden der Angst}

Ausgehend von den verschiedenen Angstindikatoren lassen sich verschiedene Messinstrumente oder -methoden finden, um Angst möglichst objektiv zu erfassen. Dies ist die apparative Messung auf der physiologischen Ebene, die Erhebung von Selbstauskünften auf der verbal-subjektiven Ebene und die Verhaltensbeobachtung auf der motorischen Ebene (vgl. SÖRENSEN 1994, 108).

\footnotetext{
${ }^{16}$ Also z.B. EEG- oder Hautwiderstandsmessung.
} 


\subsubsection{Physiologische Messverfahren}

Die physiologischen Messverfahren ermitteln die unter 2.1.3 erwähnten physiologischen Begleiterscheinungen emotionaler Prozesse und schließen von diesen gewonnenen Daten aus auf Angst. Diese Art der Messungen ist die objektivste und genaueste, sie ist unabhängig von verbalen Verständnis- oder Ausdrucksschwierigkeiten. Die heutige Technik ermöglicht weiterhin in der Regel eine kontinuierliche, parallel zum Angsterleben stattfindende Aufzeichnung von Messdaten (vgl. HACKFORT/SCHWENKMEZGER 1989, 58). Einen weiteren Vorteil stellt die Sicherheit vor Verfälschungen dar, da die getestete Person in der Regel keine oder nur geringe Kenntnisse darüber hat, was eigentlich getestet wird (vgl. SÖRENSEN 1994, 109).

Der schnelle (und ausschließliche) Gebrauch von physiologischen Indikatoren zur Angstmessung ist allerdings nicht immer ratsam: So werden physiologische Reaktionen wie z.B. die Erhöhung der Herzfrequenz nicht nur von der Emotion Angst, sondern auch von der Empfindung der Freude ausgelöst. Im konkreten Anwendungsfall der Sportpsychologie ist weiterhin zu beachten, dass physiologische Indikatoren wie Herz- und Atemfrequenz und sogar biochemische Indikatoren (Hormon- und Laktatwerte) in Momenten der sportlichen Tätigkeit viel größere Änderungen durch die physische Aktivierung als durch die angstauslösende Situation erfahren können (vgl. HACKFORT/SCHWENKMEZGER 1989, 59). Neuere Untersuchungen betrachten hier das Verhältnis der Hormone Adrenalin und Noradrenalin im Körper. Hohe Adrenalinwerte werden dabei physischer Anstrengung, erhöhte Noradrenalinwerte mentalem Stress bzw. Angst zugeordnet (vgl. DEINZER et al. 1997, 507f). Eine Situation wie die des Fallschirmspringens würde man nun mit eher erhöhten Noradrenalinwerten beschreiben wollen. Die Untersuchung von ANFILOGOFF et al. 1987 konnte diese Erhöhung zwar bestätigen, jedoch stiegen die Adrenalinwerte um einen noch größeren Faktor, obwohl es sich hierbei um Sprünge mit automatischer Auslösung ${ }^{17}$ handelte. Das Verhältnis von Adrenalin- zu Noradrenalinkonzentration änderte sich hier von 0,28:1 in Ruhe zu 0,52:1 beim Absprung (vgl. ANFILOGOFF et al. 1987, 415). Somit sind diese neueren Überlegungen womöglich nicht auf das Fallschirmspringen anwendbar.

\footnotetext{
${ }^{17}$ Sprünge, bei denen eine eher geringe physische Beanspruchung auftritt, da keine bzw. nur eine sehr kurze Freifallphase vorkommt - der Fallschirm ist ca. 3-5 Sekunden nach Verlassen des Flugzeuges bereits voll geöffnet (vgl. SCHÄFER 1998, 44; MAUSHAKE 1987, 46).
} 
Weiterhin zeigt die anscheinend in Untersuchungen bevorzugt gemessene Herzfrequenz ${ }^{18}$ häufig keinen Zusammenhang mit der erlebten Erregung (vgl. MALMSTROM 1965, 546; LAZARUS-MAINKA/SIEBENEICK 2000, 64). Andererseits ergaben aber Untersuchungen von PARFITT und HARDY 1988 mehrfach, dass der zeitliche Verlauf der Herzfrequenz dem der somatischen Erregung folgt (vgl. PARFITT et al. 2000, 47).

Durch unterschiedliche Reaktionsgeschwindigkeiten bei der Angstverarbeitung kommt es aber auch innerhalb des Körpers zu erheblichen zeitlichen Differenzen, etwa zwischen im EEG messbaren Reaktionen der Gehirnströme (in der Größenordnung von einigen Millisekunden) über Änderungen im Bereich des peripheren Kreislaufs, z.B. des Hautwiderstands (in der Größenordnung von Sekunden oder gar Minuten), bis zu biochemischen Reaktionen (Hormonausschüttung), deren Reaktionszeiten bei mehreren Minuten liegen und bis hin zu Stunden wachsen können. Durch diese möglichen zeitlichen Verschiebungen können bereits verschiedene Ergebnisse innerhalb der physiologischen Messungen stark voneinander abweichen (vgl. HACKFORT/SCHWENKMEZGER 1989, 63).

Abschließend ist beim Vergleich mehrerer physiologisch getesteter Probanden auch stets mit erheblichen Abweichungen der Messwerte zu rechnen, da jede Person qualitativ und quantitativ unterschiedlich körperlich auf einen Angstreiz reagiert: Eine Person reagiert womöglich auf Angst mit Erhöhung der Herzfrequenz, eine andere mit erhöhter Schweißabsonderung, eine dritte mit Zunahme des Blutdrucks (vgl. LAZARUS-MAINKA/SIEBENEICK 2000, 66).

\subsubsection{Messungen auf der verbal-subjektiven Ebene}

Auf der verbal-subjektiven Ebene können sowohl Bestimmungen des aktuellen Angstzustands einer Person als auch ihrer Ängstlichkeit als Persönlichkeitsmerkmal gemacht werden. Daten können hier über Selbsteinschätzungen und -beschreibungen, über Eigenschaftslisten und über Fragebogenverfahren gewonnen werden. Hierbei stellen Selbsteinschätzungen spontane Sprache und Erzählung des Angsterlebens dar, dies ist allerdings schwer auszuwerten (vgl. LAZARUS-MAINKA/SIEBENEICK 2000, 40). Häufiger werden stattdessen Selbsteinschätzungen auf Angstskalen oder ähnlichen Schätzinstrumenten durchgeführt.

\footnotetext{
${ }^{18}$ Vermutlich, da sie von den physiologischen Größen am einfachsten zu messen ist.
} 
Eigenschaftslisten sind Listen von Adjektiven in bipolarer Form. Jedem Adjektiv steht also sein entgegengesetztes gegenüber. Die zu testende Person markiert nun diejenigen Adjektive (bzw. einen Skalenwert zwischen zwei Wörtern), die den augenblicklichen oder allgemeinen Zustand am besten beschreiben (vgl. SÖRENSEN 1994, 111).

Fragebogen zur Messung von Angst sind eine sehr häufig angewandte Methode. Einzelne Items eines Fragebogens können hier unterschiedliche Angstarten oder Angstneigungen messen. Die Vorteile eines Fragebogentests liegen in der konkreten Anpassbarkeit an die Angstart, die gemessen werden soll. Die Anzahl der Bereiche oder Situationen, in denen Angst erlebt werden kann, ist unendlich groß. So gibt es eine Vielzahl „fertiger“ und auf ihre Validität überprüften Fragebogen zum Messen von Angstsituationen wie Sportangst (z.B. CSAI-2), Prüfungsangst, Schulangst, aber auch zur allgemeinen Ängstlichkeit (Trait-Skala des STAI $^{19}$ ) (vgl. LAZARUS-MAINKA/SIEBENEICK 2000, 42f).

Beim Gebrauch von Fragebögen ist zu beachten, dass die Beantwortung von Fragen einer Angstskala ebenfalls nie das tatsächliche Befinden oder Verhalten einer Person erfassen, sondern nur das Bild vermitteln kann, welches die Person von sich selbst hat oder haben will. Womöglich scheut der Proband auch davor zurück, negativ behafteten Äußerungen zuzustimmen, obwohl sie seinen momentanen Zustand treffend beschreiben. Ähnliche Fehler können durch bewusstes „Suchen nach einer richtigen oder falschen Antwort“ entstehen. Ein Verbergen von „guten und schlechten“ Antworten für den Testausgang ist bei der Entwicklung von Fragebögen äußerst schwierig und problematisch (vgl. APITZSCH 1983, 112). MARTENS kombiniert daher z.B. die Anleitung zum Ausfüllen des CSAI-2 Fragebogens für den Probanden mit „Anti-Social Desirability Instructions“, Anweisungen, die dazu motivieren sollen, Angst als etwas Positives zu sehen und ehrlich Angstzustände im Fragebogen „Zuzugeben“(vgl. MARTENS et al. 1990, 52).

\subsubsection{Verhaltensbeobachtung}

Bei der Verhaltensbeobachtung wird der Proband hinsichtlich Angstindikatoren auf motorischer Ebene (in der Regel von mehreren Betrachtern gleichzeitig) beobachtet. Das Verwenden von mehreren Beobachtern empfiehlt sich, da so Deutungsfehler in der Betrachtung eingegrenzt werden können. Daten aus Verhaltensbeobachtungen können in der Psychologie ebenso wie Daten der verbal-subjektiven Ebene zur Gewinnung von Aussagen über die

\footnotetext{
${ }^{19}$ State-Trait Anxiety Inventory von SPIELBERGER.
} 
allgemeine Ängstlichkeit oder zur Beschreibung der Zustandsangst verwendet werden (vgl. LAZARUS-MAINKA/SIEBENEICK 2000, 57).

Allerdings gelten für die Nutzung der Verhaltensbeobachtung für die Angstmessung ähnliche Einschränkungen hinsichtlich der Eindeutigkeit wie für die physiologischen Messverfahren: Ein bestimmtes beobachtetes Verhalten lässt eben nicht immer den eindeutigen Schluss auf einen Angstzustand $\mathrm{zu}^{20}$ (vgl. HACKFORT/SCHWENKMEZGER 1989, 61). Um eventuelle Fehlinterpretationen ausschließen zu können, bedarf es hier also einiger Zusatzinformationen.

Andererseits ist eine typische beobachtbare motorische Angstreaktion die Vermeidung oder die Flucht vor der Gefahr. Solches Vermeidungs- oder Fluchtverhalten ist gerade im Sport einfach und oft zu beobachten ${ }^{21}$ (vgl. HACKFORT/SCHWENKMEZGER 1989, 63).

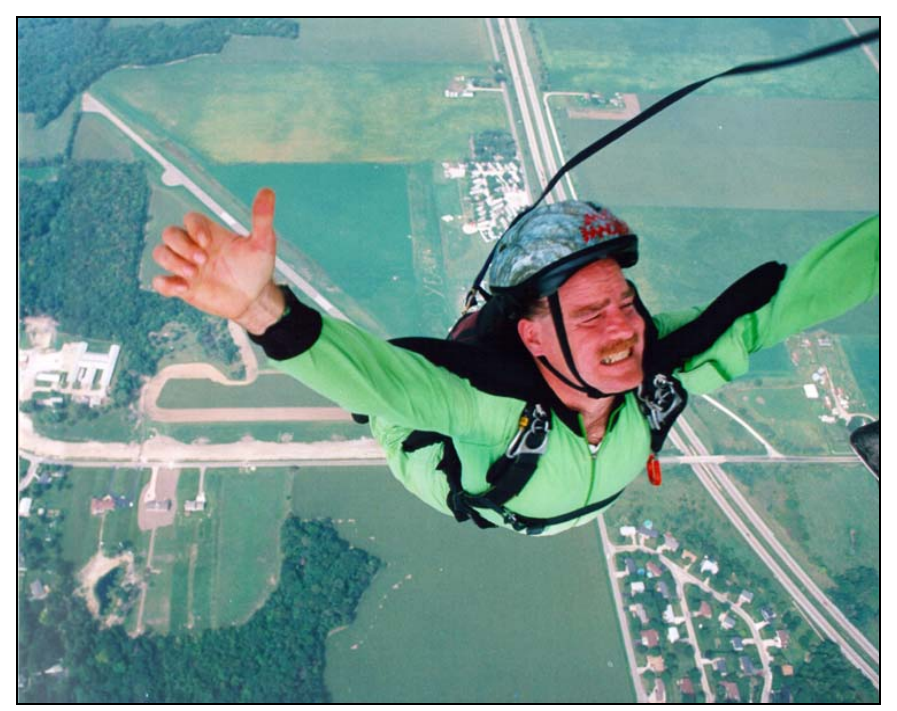

Abbildung 3: Typisches Angstgesicht (Foto: Rook Nelson).

Im Hinblick auf diese Einschränkungen erscheint die Mimik einer der am besten geeigneten motorischen Angstindikatoren zu sein. Das „typische Angstgesicht“ weist universelle, kulturunabhängige Signale auf, die von Unbeteiligten jederzeit erkannt werden können (vgl. LAZARUS-MAINKA/SIEBENEICK 2000, 59).

\footnotetext{
${ }^{20}$ Ein Skifahrer, der zitternd am Beginn einer steilen Piste steht, kann Angst haben. Er kann aber auch einfach nur frieren.

${ }^{21}$ Man denke nur an die Häufigkeit von Krankmeldungen oder „Vergessen des Sportzeugs“ im schulischen Sportunterricht.
} 


\subsubsection{Korrelationen der Methoden}

Ein wichtiger Grund, warum Angsttheorien immer wieder nicht vollends befriedigende Ergebnisse liefern können, liegt in der niedrigen Kovariation der verschiedenen Messmethoden untereinander bei empirischen Untersuchungen: Subjektiv-verbale Daten, physiologische Daten und Daten, die aus Verhaltensbeobachtungen gewonnen wurden, zeigen in der Regel keine (gelegentlich sogar negative) Korrelationen - selbst dann, wenn in der Untersuchung innerhalb der subjektiv-verbalen Daten oder Verhaltensdaten signifikante Veränderungen auftreten (vgl. HACKFORT/SCHWENKMEZGER 1989, 63; LAZARUS-MAINKA/ SIEBENEICK 2000, 66) oder wenn alle Daten quasi zeitgleich erhoben wurden (vgl. SöRENSEN 1994, 108).

Dies ist für den Wissenschaftler natürlich zunächst unbefriedigend. Eine mögliche Erklärung ist im unterschiedlichen zeitlichen Verlauf der physiologischen und motorischen Variablen und dem Prozess der subjektiven Wahrnehmung zu finden. Auch hier findet (wie innerhalb der physiologischen Messgrößen bereits aufgezeigt) die Reaktion auf Angstsituationen jeweils zeitlich verschoben statt. So berichten z.B. COHEN et al. (2003) von einem in der zeitlichen Abfolge ähnlichen - aber eben nicht identischen - Verlauf von Herzfrequenz und (mittels Fragebogen gemessener) somatischer Angstkomponente (vgl. CoHEN et al. 2003, 151).

Mögliche Abhilfe zur Lösung dieses Nicht-Korrelations-Problems könnte nicht das Messen von physiologischen Daten, sondern das Befragen des Probanden sein: „Wie schnell schlägt Dein Herz?“ - diese verbale Beschreibung der Körperbefindlichkeit und die subjektiven (ebenfalls verbal erfassten) Angstgefühle stehen in der erwarteten Beziehung zueinander. Allerdings beraubt man sich so der objektiv gemessenen Kontrollgröße und - schlimmer noch - der Korrelationskoeffizient z.B. zwischen der vom Probanden geschätzten und der tatsächlich gemessenen Herzfrequenz liegt bei maximal $r=0,35$ bis $r=0,40$ (vgl. LAZARUSMAINKA/SIEBENEICK 2000, 67).

\section{„Fragebogenuntersuchungen zur subjektiven Wahrnehmung physiolo- gischer Reaktionen sagen praktisch nichts über tatsächliche inter- individuelle Unterschiede in physiologischen Reaktionen aus. “} (in: ASENDORPF 1996, 137). 
Von trainierten Sportlern sollte man andererseits aber erwarten können, dass Körpergefühl und Selbstbeobachtungsfähigkeit deutlich besser ausgeprägt sind und solche Abweichungen nicht so stark ausfallen.

Diese These lässt sich durch Untersuchungen von PARFITT (1988) und HARDY und PARFITT (1987) bestätigen. Beide fanden, dass die Herzfrequenz von Sportlern zeitlich den selben Verlauf wie die somatische Angstkomponente hatte (vgl. PARFITT et al. 2000, 47).

In der Messung von Angst läuft es so letztendlich darauf hinaus, möglichst alle drei Messmethoden miteinander zu kombinieren, auch wenn damit zu rechnen ist, dass sie zeitlich asynchron verlaufen. Im Rahmen der eindeutigen Zuordnung von physiologischen oder motorischen Indikatoren werden ohnehin bereits Zusatzinformationen aus einer anderen Messebene benötigt. Das Messinstrument des Fragebogens ist aus Gründen der Validität und Reproduzier- und Vergleichbarkeit quasi unverzichtbar (vgl. LAZARUS-MAINKA/SIEBENEICK 2000, 69) - sollte aber z.B. mit einzelnen physiologischen Messgrößen oder mit Kombinationen dieser Werte verglichen und erweitert werden (vgl. MULLEN/HARDY 2000, 797f).

\subsubsection{Eigene Messmethoden für die Sportangst?}

Wie bereits in 2.2.2 beschrieben, existieren Angstmessinstrumente (Fragebögen) für eine Vielzahl möglicher Anwendungen und Fragestellungen. Mit dieser Menge an „speziellen“ Angstfragebögen soll eine mögliche situationsspezifische Angstabhängigkeit von Ängstlichkeit oder Zustandsangst erfasst werden. Diese Tendenz zur Entwicklung spezieller Angstfragebögen war selbstverständlich auch in der Sportpsychologie willkommen, MARTENS entwickelte im Jahr 1977 den ersten Sportangst-Fragebogen SCAT ${ }^{22}$ (vgl. HACKFORT/ SCHWENKMEZGER 1989, 66).

Die Notwendigkeit eines solchen Spezialinstruments wird allerdings mehrfach in Frage gestellt: SCHWENKMEZGER zeigt 1981, dass die Trait-Skala des STAI-Fragebogens die Eigenschaftsangst genauso gut beschreibt wie der SCAT von MARTENS (vgl. HACKFORT/ SCHWENKMEZGER 1989, 66).

In ihrer Meta-Analyse zum Zusammenhang von Angst und sportlicher Leistung untersuchten KLEINE und SCHWARZER 1991 auch evtl. Abhängigkeiten der gemessenen Werte vom verwendeten Angstfragebogen. Sie fanden heraus, dass sportspezifische Fragebögen wie der

\footnotetext{
${ }^{22}$ Sport Competition Anxiety Inventory.
} 
SCAT oder CSAI keine besseren Leistungsvorhersagen ermöglichten als Fragebögen zur Erfassung allgemeiner Angst wie der STAI (vgl. KLEINE/SCHWARZER 1991, 24).

Vor dem Hintergrund der Nicht-Homogenität der vielen unterschiedlichen Sportarten stellt sich weiterhin die Frage, ob ein einziger Sportangst-Fragebogen überhaupt ausreichend sein kann, um gleichzeitig die Differenzen zwischen verschiedenen Sportarten, Situationen des Sporttreibens und der unterschiedlichen Sportlertypen zu erfassen. Zusätzlich ist es möglich, dass ein sportspezifischer Fragebogen zwar in der Lage ist, für den Sport typische Stressoren besser zu erfassen, dabei aber womöglich Angst oder Stress ausblendet, welche nicht auf die sportliche Situation zurückzuführen ist (vgl. RAGLIN/HANIN 2000, 110).

Eine Verwendung sportspezifischer Bögen mag so also zwar im Sinne der Lesbarkeit und besseren Identifikation des Probanden mit dem Fragebogeninhalt praktisch sein, ist jedoch nicht zwingend notwendig. 


\section{Angst und sportliche Leistung}

Der sportliche Wettkampf stellt eine der typischen Angstsituationen schlechthin dar: Im Wettkampf wird für Sportler, Kameraden, Gegner, Schiedsrichter, Trainer und Zuschauer schonungslos die eigene Rangposition, Kompetenz und Leistungsfähigkeit offengelegt. Der Ausgang eines Wettbewerbes entscheidet in der Regel über die Zukunft des Sportlers: Anhand der sportlichen Leistung in der Angstsituation wird das Urteil über die Erfüllung des Selbstbildes, über die Position innerhalb der Mannschaft, die Notwendigkeit und den Umfang zukünftigen Trainings oder gar über den sportlichen „Ruhestand“ gefällt (vgl. LAZARUS 2000, 244).

Der Zusammenhang zwischen Angst und Leistung ist allerdings bis jetzt noch nicht abschließend erklärt worden. Zwar existieren viele Modelle, die mögliche Abhängigkeiten beschreiben und Erklärungstheorien bieten. Allerdings handelt es sich hier stets ausdrücklich um Modelle, die jeweils nur versuchen können, den „unsichtbaren“ Zusammenhang zwischen den „sichtbaren“ Größen des äußeren Reizes und einer sichtbaren Aktion eines Probanden zu beschreiben.

\subsection{Angst-Leistungs-Modelle}

Im Folgenden wird zunächst ein Überblick über die Vielzahl der in der Literatur zu findenden Theorien gegeben, die versuchen, den Zusammenhang von Angst und sportlicher Leistung zu erklären:

\subsubsection{Drive-Theorie}

Die Drive-Theorie ${ }^{23}$ (nach Hull 1943 und modifiziert von Spence und Spence 1966) sagt einen positiven linearen Zusammenhang von Angst und Leistung voraus.

Traditionsgemäß bezieht sich die Theorie dabei vornehmlich auf die physiologische Erregung. Bei einer Erhöhung der Erregung und Aktivierung steige nach der Drive-Theorie die Wahrscheinlichkeit der Anwendung eines „dominanten“ Verhaltens aus der Menge der möglichen Reaktionen. Dabei sollten folgende Eigenschaften beobachtbar sein:

\footnotetext{
${ }^{23}$ Auch Trieb-Theorie genannt.
} 
1. Die Stärke einer Reaktion kovariiert mit der Intensität des Angstreizes.

2. Die Reaktionen passen sich den Reizen an, bei einer Reihe von (gleichen) Angstreizen nehmen die Reaktionen ab. Es wird also eine Art Angstkonditionierung postuliert.

3. Die Reaktionsstärke auf Angstreize ist interindividuell verschieden (vgl. LAZARUSMAINKA/SIEBENEICK 2000, 279).

Für gut beherrschte oder recht einfache Aufgaben sollten Angstreize also zu einer Verbesserung der Leistung führen. Ob diese Einschränkung noch die Anwendbarkeit der Drive-Theorie für komplexe sportliche Bewegungen zulässt, ist eher fraglich (vgl. WEINBERG 1989, 97). Andererseits finden sich in der Literatur vereinzelte Beispiele, die die DriveTheorie für sportliche Bewegungen gültig erscheinen lassen: LOW und MCGRATH untersuchten 1971 den Zusammenhang von Angst und Leistung bei Baseballspielern und fanden einen positiven, linearen Zusammenhang von Aktivierung und Leistung für den Fall, dass die Aufgabenschwierigkeit konstant gehalten wird (vgl. Low/MCGRATH 1971, 1167 zit. nach HäCKER 1983, 53). Demgegenüber gelang es auch KLEINE und SCHWARZER in ihrer Meta-Analyse 1991 nicht, einen positiv linearen Zusammenhang von Angst und sportlicher Leistung nachzuweisen (vgl. Kleine/Schwarzer 1991, 24). Auch NoteboOm et al. (2001) wiesen eher eine Verschlechterung der Leistung bei einer Erhöhung der Angstwerte nach (vgl. NoteBoom et al. 2001, 2100).

\subsubsection{Inverted U-Modell}

Die umgekehrt U-förmige Hypothese von YERKES/DODSON (1908) bietet eine alternative Erklärung des Angst-Leistungs-Zusammenhangs. Sie sagt aus, dass die Leistung mit wachsender Angst bis zu einem bestimmten Punkt zunehme. Ab diesem Punkt führt eine weitere Steigerung der Angst zu einem Abnehmen der Leistung, sodass im Bereich eines mittleren Angstwertes die Leistung maximal ist (vgl. HumARA 1999). Diese Hypothese, ursprünglich an Mäusen getestet, wurde von Sportwissenschaftlern „dankbar“ angenommen vor allem wohl, da sie logisch und anschaulich erscheint und z.B. die Vorhersage eines „optimalen Angstniveaus“ zur praktischen Anwendung in Training oder Wettkampf ermöglicht $^{24}$ (vgl. WEINBERG 1989, 99).

\footnotetext{
${ }^{24}$ Siehe hierzu z.B. praktische Handanweisungen zum Selbstgebrauch bei CLANCY 1999, 18f.
} 


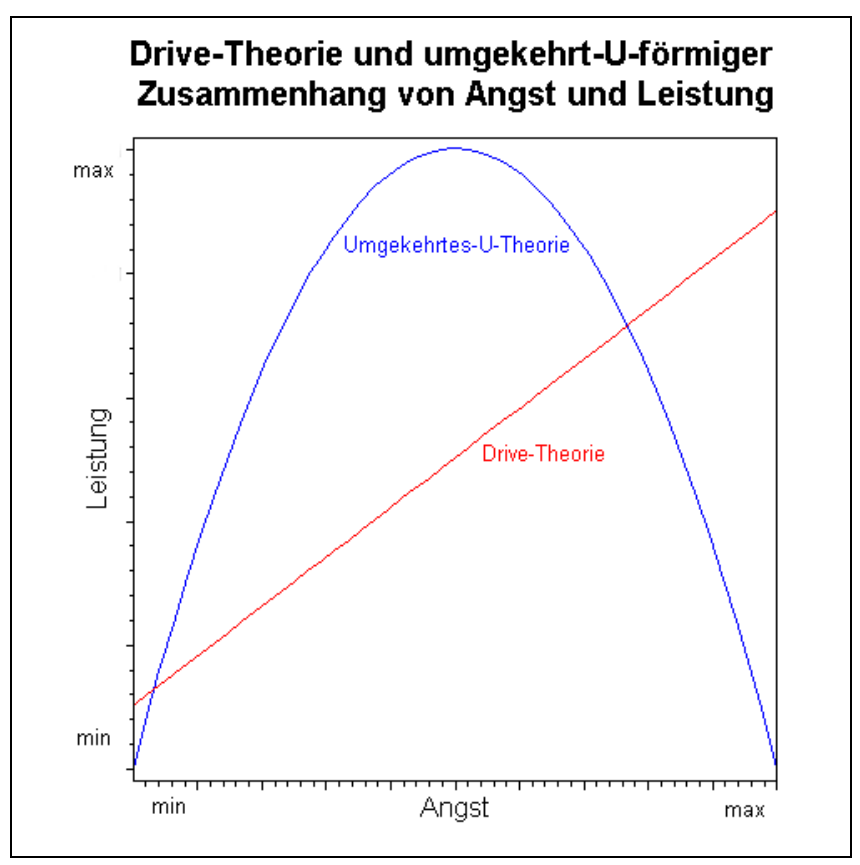

Abbildung 4: Angst/Leistung bei Drive- und umgekehrter U-Theorie (vgl. WEINBERG 1989, 99).

Eine der populärsten Untersuchungen, die das umgekehrt U-förmige Modell bestätigen, ist die Forschungsreihe von FENZ und EPSTEIN 1972 zur Angst und Leistung bei Fallschirmspringern. Sie zeigten, dass Springer, die kurz vor dem Absprung ihre hohe Erregung senkten $^{25}$, sehr gute Leistungen im Sprung erbrachten. Diejenigen Springer, die mit deutlich höherer Erregung das Flugzeug verließen, wurden in ihrer Leistung als schlecht bewertet. Dieser Zusammenhang war unabhängig vom Erfahrungsstand der Fallschirmspringer beobachtbar (vgl. FENZ/JONES 1972, 2f; WEINBERG 1989, 101).

Der klare Nachweis der umgekehrt U-förmigen Beziehung zwischen Angst und Leistung steht indessen noch aus - es finden sich nämlich ebenso viele Untersuchungen, die das Inverted-UModell widerlegen, wie solche, die eine Bestätigung des Modells ergeben (vgl. KLEINE/ SCHWARZER 1991, 24). So wird als Kritik am Inverted-U-Modell angeführt, dass alle Untersuchungen, die das Modell bestätigen, jeweils statistische Besonderheiten oder Anomalien (vgl. KLEINE/SchWARZER 1991, 25) oder Unachtsamkeiten im Versuchsdesign z.B. hinsichtlich der Werteeinteilung aufweisen ${ }^{26}$ (vgl. HÄCKER 1983, 46f).

\footnotetext{
25 Etwa durch gezieltes mentales Training während des Steigfluges auf Absprunghöhe (vgl. MACHAC/ Machacova 1994, 75; Biscoe 1999, 13; WiLliams/HarRis 1998, 225ff).

${ }^{26}$ So unterscheiden die kritisierten Versuche nur zwischen drei Aktivierungswerten (niedrig, mittel, hoch) und zwischen zwei Leistungswerten (niedrig, hoch). So erhält man 6 mögliche Einzelergebnisse oder „Freiheitsgrade“. Dies sind aber so wenig, dass nur zwei der möglichen Kombinationen das Modell nicht bestätigen.
} 
Weitere Kritik erfährt das Inverted-U-Modell, da es weder zwischen Angst als Zustand und Ängstlichkeit als Eigenschaft noch zwischen den Einflüssen unterschiedlicher Zustandsarten der Angst (kognitiv und somatisch) unterscheiden kann.

Eine Untersuchung von TAYLOR (1987) könnte diesen Vorwurf allerdings entkräften: Er zeigte, dass das umgekehrt U-förmige Modell jeweils für kognitive, somatische Angst und Selbstwirksamkeit den Zusammenhang von Angst und Leistung richtig beschrieb - dieser Nachweis gelang allerdings nur für als feinmotorisch und anaerob klassifizierte Sportarten ${ }^{27}$ (vgl. TAYLOR 1987, 150).

Ferner kann das Inverted-U-Modell auch nicht den Grund für den postulierten Kurvenverlauf der Angst-Leistungs-Kurve erklären (vgl. WeINBERG 1989, 101; JONES 1995, 451).

\subsubsection{Multidimensionales Modell}

MARTENS (1990) erweiterte die vorhandenen Theorien in eine multidimensionale Richtung, um die Einflüsse verschiedener Angstkomponenten auf die Leistung berücksichtigen zu können ${ }^{28}$. Man hatte bei vielen früheren Untersuchungen bereits den zeitlichen Verlauf von Angstkomponenten bzw. Messgrößen betrachtet und unterschiedliche Verläufe von somatischer und kognitiver Angst bzw. Erregung festgestellt (vgl. JONES 1995, 458).

Beide Größen ließen sich separat manipulieren und sollten nach MARTENS dann auch im Zusammenhang von Angst und Leistung berücksichtigt werden. Die kognitive Angstkomponente hat hierbei einen negativ linearen und die somatische Angstkomponente einen schwachen, umgekehrt U-förmigen Zusammenhang mit der Leistung. Ferner wird ein positiv linearer Zusammenhang zwischen Selbstwirksamkeitüberzeugung und der Leistung vorausgesagt (vgl. KERR 1997, 97).

Trägt man die beiden Angstkomponenten additiv auf, so erhält man eine Angst-Leistungsoberfläche wie in Abbildung 5 auf der folgenden Seite gezeigt.

\footnotetext{
27 TAYLOR untersuchte in dieser Kategorie alpines Skifahren, Tennis, Basketball und einige leichtathletische Disziplinen (vgl. TAYLOR 1987, 145).

${ }^{28}$ Das Inverted-U-Modell ist zweidimensional - sobald Angst aber als mehrdimensional betrachtet wird, reichen diese zwei Dimensionen nicht mehr aus und müssen um eine dritte erweitert werden: Man erforscht Leistungsoberflächen im dreidimensionalen Raum (vgl. HARDY/HAGTVET 1996, vi).
} 


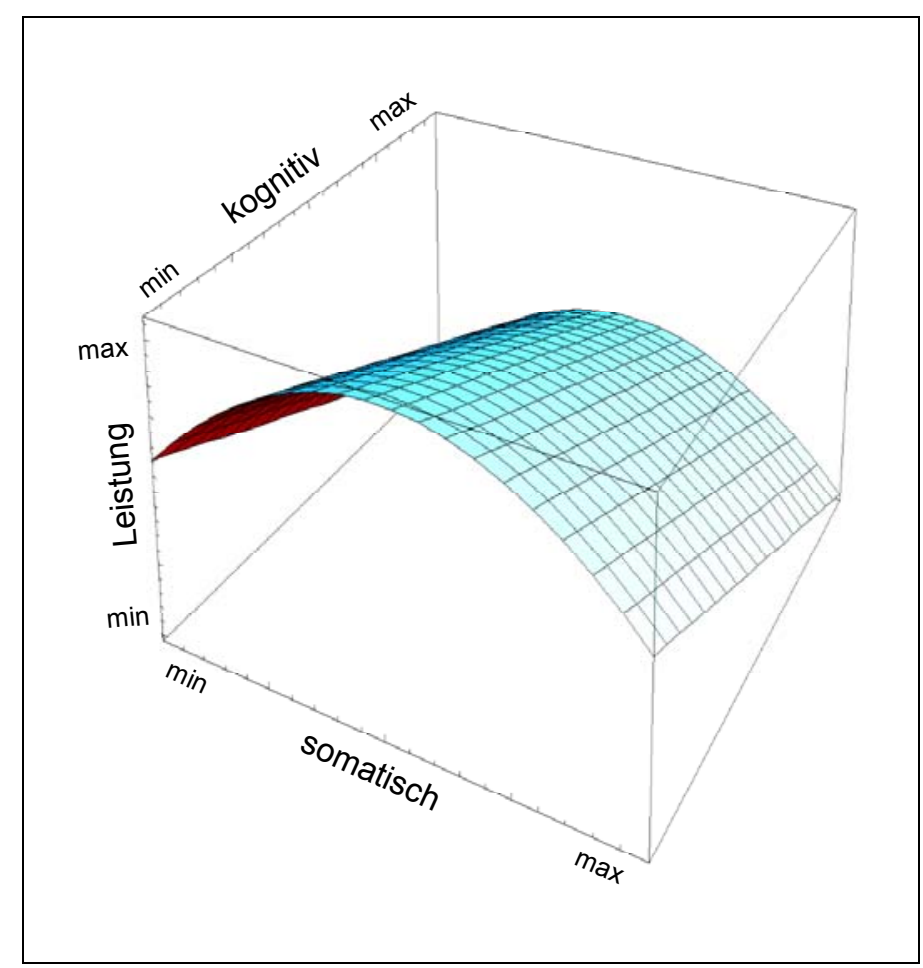

Abbildung 5: Additive Darstellung somatischer (umgekehrtes-U) und kognitiver (negativ linearer) Angstkomponente (eigene Darstellung).

Diese drei postulierten Zusammenhänge wurden unter anderem in einer Untersuchung von BURTON 1988 bestätigt (vgl. JONES 1995, 461) - allerdings waren andere Wissenschaftler auch weniger erfolgreich und konnten entweder nur den umgekehrt U-förmigen Zusammenhang von somatischer Angst und Leistung (vgl. JONES 1995, 462) oder die negativ-lineare Beziehung von kognitiver Angstkomponente und Leistung nachweisen (vgl. GRASSO 2000, $14 f)$.

Kritisiert am multidimensionalen Angstmodell von MARTENS wird weiterhin, dass die jeweiligen Angstkomponenten als unabhängig voneinander angenommen werden. So erscheint es logisch, dass ein Sportler, der in hohem Maße kognitiv besorgt (also auch erregt) ist, nur niedrigere Werte in seiner Selbstwirksamkeitsüberzeugung erreicht. Auf eine ähnliche Weise könnte bereits das Empfinden und Erleben physiologischer Erregung zu einer Zunahme der Besorgnis führen. Durch diese Nicht-Berücksichtigung von möglichen Abhängigkeiten der einzelnen Angstwerte untereinander würde es zu deutlichen Verfälschungen des Angstmodells kommen (vgl. MCNALLY 2002). 
In der Tat sind solche Abweichungen vom multidimensionalen Angstmodell nachgewiesen. JEROME und WiLLIAMS konnten 2000 keine Bestätigung für das multidimensionale oder für einfachere Modelle erbringen (vgl. JEROME/WILLIAMS 2000, 248).

Weiterhin zeigten HARDY und PARFITT 1991, dass die Leistung mit zunehmender kognitiver Erregung auch durchaus besser werden kann: Kognitiv Hochängstliche erbrachten sowohl sehr gute als auch sehr schlechte Leistungen (vgl. HARDY 1997, 279; HARDY/PARFITT 1991, 173; JONES 1995, 462).

Eine mögliche Begründung für diese Abweichungen liefern PARFITT/PATES: Sie führen an, dass verschiedene Angstkomponenten auch unterschiedliche Leistungs- oder Anforderungskomponenten beeinflussen, und zwar in Abhängigkeit von der motorischen Anforderung (vgl. PARFITT/PATES 1999, 351). Siehe hierzu auch Abschnitt 3.2.2.1 auf Seite 41.

\subsubsection{Zone of Optimal Functioning}

Das Modell der Zone des optimalen Funktionsniveaus (ZOF) von HANIN (1980) wird oft fälschlicherweise als Ergänzung oder Umformulierung des Inverted-U-Modells beschrieben. Dies ist jedoch nicht korrekt. Entstanden ist dieses Modell, um möglichst präzise das optimale Erregungsniveau eines Sportlers vorhersagen zu können. Auch das Modell der Zone des optimalen Funktionsniveaus sagt einen Punkt der optimalen Erregung voraus. Dieser Punkt kann aber - von Proband zu Proband verschieden - irgendwo im Bereich der Angstskala zu finden sein. Einige Individuen werden also ihre optimale Erregung bei niedrigen, andere bei mittleren oder hohen Angstwerten haben (vgl. RAGLIN/HANIN 2000, 99ff; KERR 1997, 94; MORGAN/ElLiCKSON 1989, 168). Für jeden Sportler gäbe es dann eine individuell verschiedene Angstzone, in der seine Leistung maximal sein wird. SALMINEN et al. fanden in der Tat bei einer Untersuchung von 253 Athleten optimale Angstwerte von 26 bis 67 auf der State-Angstskala des STAI, die von 20 bis 80 Punkten reicht (vgl. SALMINEN et al. 1995, 725). Liegt der Sportler vor dem Erbringen einer Leistung außerhalb seiner „Optimalzone“, so wird nach HANIN die Leistung abnehmen.

Aussagen und Untersuchungen finden also auf das Individuum und nicht auf die Gruppe bezogen statt, indem zunächst der Proband seine Angstwerte (rückwirkend) für eine Situation, in der er optimale Leistung erbracht hat, erfassen lässt. Hierbei bietet sich z.B. die Nutzung 
des CSAI-2-Fragebogens oder eines anderen Bogens ${ }^{29}$, der mehrere Komponenten der Zustandsangst misst, an. Im Rahmen der weiteren Betreuung des Athleten werden dann weitere Punkte mit Angst- und Leistungswerten erfasst. Die Zone des optimalen Funktionsniveaus erhält man, indem zum als optimal beschriebenen somatischen Angstwert die halbe Standardabweichung addiert und subtrahiert wird. Zugrunde liegt dann hier ein multidimensionales Angst-Leistungs-Modell, bei dem somatische Angst die Leistung umgekehrt U-förmig und kognitive Angst die Leistung negativ linear beeinflusst. Deshalb erhält man für die „Höhe“ der optimalen Angstzone den Wert vom Nullwert der kognitiven Angst bis zum Wert der optimalen Leistung plus eine halbe Standardabweichung (siehe hierzu auch Abbildung 6) (vgl. Raglin/Hanin 2000, 107f; RANDLE/WeinBerg 1997, 166f).

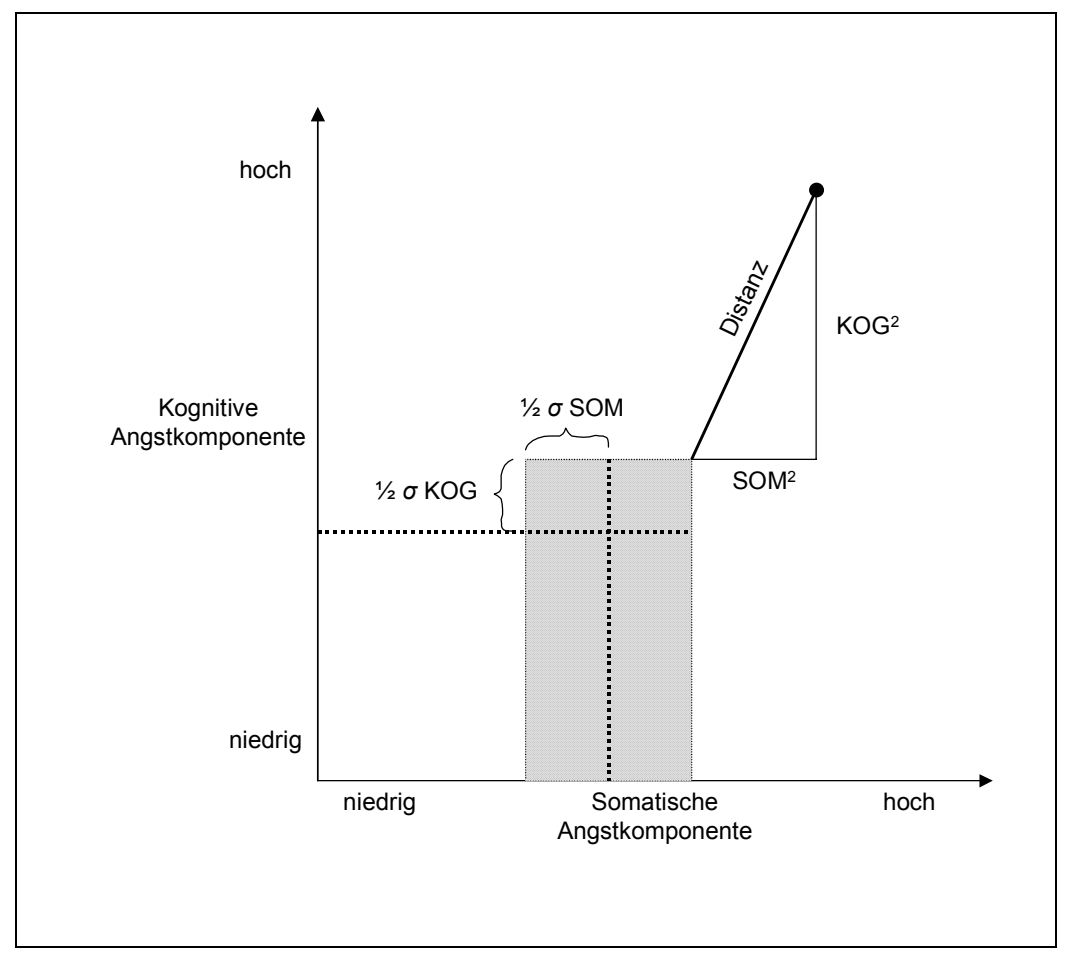

Abbildung 6: Multidimensionale angstbasierende Zone des optimalen Funktionsniveaus (vgl. RANDLE/WEINBERG 1997, 167).

In mehreren Untersuchungen, unter anderem von TURNER und RAGLIN (1991), SALMINEN et al. (1995) sowie von IMLAY et al. (1995), zeigte sich schließlich auch eine Überlegenheit des ZOF-Modells gegenüber dem Inverted-U-Modell hinsichtlich der Leistungsvorhersage bei

\footnotetext{
29 HANIN nutzte ursprünglich die State-Angstskala des STAI (vgl. RAGLIN/HANIN 2000, 99; MoRGAN/ ELLICKSON 1989, 169).
} 
bekannten Angstwerten von Athleten (vgl. TURNER/RAGLIN 1991, 119; RAGLIN/HANIN 2000, 102f; SALMINEN et al. 1995, 728; IMLAY et al. 1995, 301).

Allerdings kann dieses Modell keine Erklärung geben, warum es zu den interindividuellen Leistungsunterschieden kommt (vgl. HUMARA 1999). JONES kritisiert weiterhin, dass das ZOF-Modell wie auch das Inverted-U-Modell nur unidimensional ist, also nicht die verschiedenen Angstkomponenten berücksichtigt und dass es ursprünglich einen sportunspezifischen Fragebogen (z. B. den STAI) nutzt (vgl. JONES 1995, 453). Diese Kritik ist allerdings durch die Erweiterung von RANDLE und WEINBERG mit dem Gebrauch des CSAI-2 und der Beschreibung der Angstzone mit kognitiver und somatischer Angstkomponente nicht mehr haltbar.

Das ZOF-Modell wurde 1995 von HANIN zum IZOF-Modell (Individual Zones of Optimal Functioning) erweitert. Dabei wurde nun nicht mehr ausschließlich die Angst des Sportlers als einzige Emotion untersucht, sondern zusätzlich eine ausgewogenere Betrachtung von positiven und negativen Emotionen des Sportlers angestrebt. Somit gilt es dann wiederum, für den einzelnen Sportler die Wertezonen der Emotionen, die mit optimaler Leistung verknüpft sind, zu identifizieren und bewusst ansteuern zu können (vgl. HANIN 2000, 68f). Durch diese Erweiterung ist es nun auch u.a. möglich, die Selbstwirksamkeitsüberzeugung mit in das IZOF-Modell von Emotion und sportlicher Leistung zu integrieren. Spätestens durch diese Erweiterung wird das IZOF-Modell somit zu einer „echten“ mehrdimensionalen Herangehensweise, die auch Interaktionen zwischen den verschiedenen Einflussgrößen zulässt ${ }^{30}$ (vgl. HANin 2000, 73f; Raglin/Hanin 2000, 111). Da stets Individuen betrachtet werden, sind zusätzlich einige der im folgenden Abschnitt 3.2.1 diskutierten weiteren Einflussgrößen im IZOF-Modell enthalten.

Auch das IZOF-Modell ist dabei aber keine eigenständige Theorie zum Angst-LeistungsZusammenhang. Es vermag nur für einen einzelnen Sportler vorherzusagen, welche Emotionswerte für maximale sportliche Leistungen anzustreben wären. Es trifft aber keine Aussage, wie nun die einzelnen Emotionen die sportliche Leistung beeinflussen bzw. miteinander interagieren (vgl. RAGLIN/HANIN 2000, 109).

\footnotetext{
${ }^{30}$ Das IZOF-Modell beinhaltet zwar die positiven und negativen Emotionen, die sich auf die sportliche Leistung auswirken, sagt aber nicht, wie die jeweilige Leistungsabhängigkeit oder Interaktion ist.
} 


\subsubsection{Neuere Theorien}

Anfang der neunziger Jahre wurden die multidimensionalen Angst-Leistungs-Modelle vor allem um den Sportpsychologen HARDY (1991) nochmals verfeinert. $\mathrm{Zu}$ einer genaueren Betrachtung und Beschreibung der Theorie von HARDY sind jedoch einige Grundlagen unerlässlich. Daher erfolgt die Diskussion dieses Modells im nächsten Kapitel zur Katastrophentheorie - zunächst folgen Erweiterungen im Sinne ergänzender Parameter, die die Angst-Leistungs-Beziehung moderieren können.

\subsection{Weitere Einflussgrößen}

In nahezu allen Untersuchungen, die sich mit dem Zusammenhang von Angst und sportlicher Leistung beschäftigen, werden Eingrenzungen oder zusätzliche Größen, welche die Leistung mit beeinflussen, aufgezeigt. Diese weiteren Größen, die bewusst erforscht oder nur postuliert wurden, lassen sich in die Kategorie der Persönlichkeits- und die der sportartbezogenen Merkmale untergliedern.

\subsubsection{Persönlichkeitsmerkmale}

Mehrere Persönlichkeitsmerkmale können den Zusammenhang zwischen Angst und sportlicher Leistung beeinflussen. Dies sind die Eigenschaftsangst (oder Ängstlichkeit), die Selbstwirksamkeit (als ,positive“ kognitive Angstkomponente), das Leistungsniveau des Sportlers, das Geschlecht und Angstbewältigungsstile (Coping-Stile).

\subsubsection{Eigenschaftsangst}

Da die Eigenschaftsangst bzw. die Ängstlichkeit einer Person aussagt, wie stark die Reaktion auf einen angstauslösenden Reiz sein wird, ist hierdurch auch sofort eine Abhängigkeit der (sportlichen) Leistung von der Ängstlichkeit zu vermuten. Im Prinzip wird unter anderem die Ängstlichkeit vorherbestimmen, wie hoch die Zustandsangst, die gewissermaßen die „Endgröße“ der Angstreaktion darstellt, sein wird. Solche Unterschiede in der Zustandsangst sind vor allem in selbstwertrelevanten Stresssituationen zu beobachten: Hochängstliche reagieren hier mit einer deutlich höheren Zustandsangst als Niedrigängstliche. Die Angst-LeistungsKurve sollte hierdurch also eine Verschiebung erfahren. 


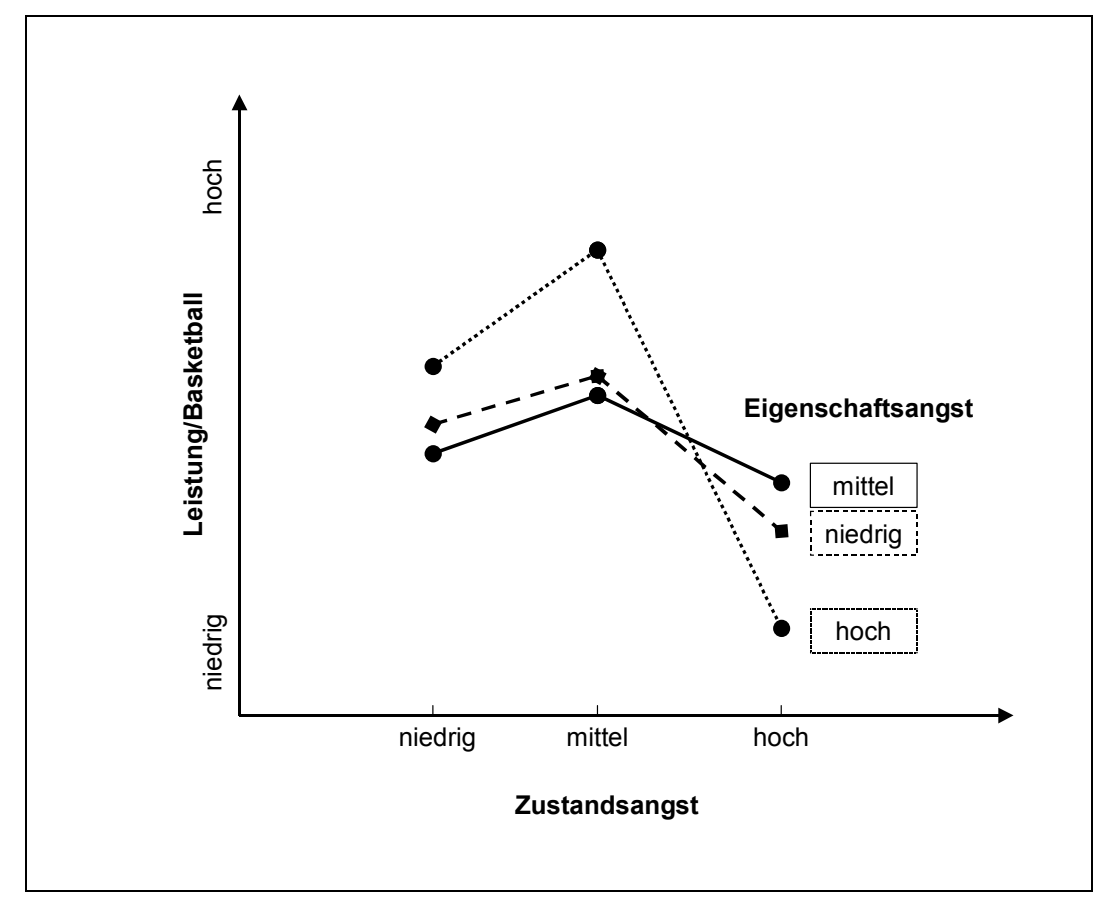

Abbildung 7: Ängstlichkeit und Leistung (vgl. SONSTROEM/BERNARDO 1982).

In zwei verschiedenen Untersuchungen konnte diese Erwartung bestätigt werden: KLAVORA untersuchte 1978 die Eigenschafts- und Zustandsangst von Basketballern, ebenso SONSTROEM und BERNARDO 1982. Beide Untersuchungen wiesen einen näherungsweise umgekehrt Uförmigen Zusammenhang zwischen Zustandsangst und sportlicher Leistung nach. Je nach der personenspezifischen Ängstlichkeit sind die Angst-Leistungs-Kurven in Richtung der Zustandsangst verschoben (vgl. ALLMER 1981, 520).

In Abbildung 7 erkennt man, dass dabei interessanterweise das optimale Erregungsniveau für Hochängstliche bei höheren Angstwerten liegt als bei Niedrigängstlichen (SONSTROEM/ BERNARDO 1982, zit. nach ZIMMERMANN 2001, 78).

Indirekte Kritik erfährt diese Aussage unter anderem von GLANZMANN (1985): Er führt an, dass Hochängstliche bei Angsterhebung nach dem Angsterleben ${ }^{31}$ tendenziell dazu zu neigen scheinen, zu hohe Zustandsangstwerte anzugeben. Dieses „Übertreiben“ der Angstangabe mag daran liegen, dass Hochängstliche hier versuchen, eventuelle Leistungs- oder Verhaltens-

\footnotetext{
${ }^{31}$ Nahezu alle von mir gesichteten Untersuchungen erheben jedoch die Angstwerte nach dem Moment des Angsterlebens - so ergab sich in der Meta-Analyse von KLEINE und ScHWARZER 1991 ein „,besserer“ AngstLeistungs-Zusammenhang, wenn der Angstwert erst nach der Leistungsprüfung erhoben wird (vgl. KLEINE/ SCHWARZER 1991, 19; SÖRENSEN 1993, 80).
} 
defizite von vornherein $\mathrm{zu}$ begründen. GLANZMANN führt hierzu folgende mögliche Argumentationskette eines Hochängstlichen an:

„Es könnte sein, daß der Experimentator von meinen Leistungen eben im Experiment enttäuscht ist. Daher werde ich ihm jetzt zeigen, wie schlecht es mir beim Experiment ging, damit er nicht von mir enttäuscht ist." (in: GLANZMANN 1985, 172).

Mitunter werden Hochängstliche sogar von sich selbst vor dem Beginn einer Aufgabensituation ein Scheitern erwarten - und dann wegen der negativen Erwartung auch tatsächlich nur eine schlechte Leistung erbringen. Durch dieses „self-fulfilling“ lässt sich also der in Abbildung 7 erkennbare höhere Zustandsangstwert der Hochängstlichen auch als „Erhebungsartefakt" deuten.

\subsubsection{Selbstwirksamkeitsüberzeugung}

Die Selbstwirksamkeitsüberzeugung soll nach dem multidimensionalen Angstmodell von MARTENS einen positiven linearen Zusammenhang mit der sportlichen Leistung haben. Ebenso wird sie in neueren Modellen von HARDY als positiv wirkende Größe aufgeführt. Diese positive Wirkung lässt sich so deuten, dass der angsterlebende Sportler motivierter ist. Er wird seine Angst ,positiv“ erleben und aus seiner Besorgnis maximale Vorteile ${ }^{32}$ ziehen für den Fall, dass die Selbstwirksamkeit hoch genug ist. In diesem Fall wäre also im Versuch womöglich auch wieder ein positiver Zusammenhang von (kognitiver) Angst und Leistung zu beobachten (vgl. TAYLOR 1987, 140; PARFITT/PATES 1999, 355).

Dieser positive Zusammenhang von Selbstwirksamkeit und Leistung wurde von PARFITT und PATES (1999) in einer Wiederholung der Untersuchung an Basketballspielern von SONSTROEM und BERNARDO, von DURTSCHI (2001) in einer Betrachtung von Mountainbikern, von RAUDSEPP und KAIS (2002) in einer Untersuchung an Beachvolleyballspielern, von ORBACH (1999) in einer Untersuchung an Fußballern und von EDWARDS et al. (2002) in Befragungen von Sportlern aus acht verschiedenen Disziplinen eindrucksvoll bestätigt (vgl. PARFitT/PAtes 1999, 354f; Durtschi 2001, 176; RAudsepP/Kais 2002, 411; OrbaCH 1999, 874; EDWARDS et al. 2002, 7f).

\footnotetext{
32 Zum Beispiel hinsichtlich einer besseren Antizipation, Bewegungsplanung oder „Vorwegnahme“ von möglichen Situationsausgängen.
} 
Auch TAYLOR konnte den positiven Einfluss der Selbstwirksamkeitsüberzeugung auf die sportliche Leistung in seiner Untersuchung von verschiedenen Sportarten 1987 nachweisen. Dieser Zusammenhang war vor allem bei den von TAYLOR als grobmotorisch eingeordneten Sportarten $^{33}$ nachzuweisen, bei den als feinmotorisch beschriebenen Sportarten ${ }^{34}$ hatte die Selbstwirksamkeitsüberzeugung allerdings nur geringen Einfluss auf die sportliche Leistung (vgl. TAYLOR 1987, 150f).

Auffallend an der Einteilung von TAYLOR ist, dass es sich in der Gruppe der als grobmotorisch beschriebenen Sportarten ausschließlich um Individualsportarten handelt, bei der anderen Gruppe nahezu vollständig um Mannschaftssportarten. In einer Untersuchung an 136 norwegischen Olympia-Teilnehmern von KJøRMO und HALVARI (2002) wurde dieser Trend des engen positiven Zusammenhangs von Selbstwirksamkeit und sportlicher Leistung innerhalb der Individualsportarten erneut festgestellt (vgl. KJøRMO/HALVARI 2002, 963f).

Im Hinblick auf die in Abschnitt 4.3 ab Seite 60 vorgestellten komplexen Angst-LeistungsModellierungen aus der Sicht der Katastrophentheorie sei bereits jetzt angemerkt, dass die besondere positive Rolle der Selbstwirksamkeit in diesen Modellen gesondert zum Vorschein kommt: Eine hohe Selbstwirksamkeitsüberzeugung scheint nicht nur mit besseren Leistungen verknüpft zu sein, sondern kann anscheinend den Sportler davor bewahren, plötzliche Leistungsänderungen ${ }^{35}$ hinnehmen zu müssen (vgl. EDWARDS et al. 2002, 11f).

\subsubsection{Leistungsniveau}

Das Leistungsniveau des Sportlers wird in der Literatur als weitere Größe angeführt, die die Angst-Leistungs-Beziehung beeinflussen kann: So kann man vermuten, dass Anfänger in einer Sportart deutlich aufgeregter sind als Spitzensportler. Diese Aufregung kann dann dazu führen, dass weniger erfahrene Sportler und Anfänger sich mehr auf ihre Angstgefühle konzentrieren und so abgelenkt sind und eine schlechtere Leistung erbringen. Fortgeschrittene bzw. Experten hingegen sind eher aufgabenorientiert.

FENZ und EPSTEIN wiesen diese größere Aufgeregtheit bei Anfängern im Fallschirmsport in ihrer Untersuchung von 1967 nach, ebenso FALK/BAR-ELI 1995 (vgl. FENZ/EPSTEIN 1967, 41;

\footnotetext{
${ }^{33}$ Querfeldeinlauf, Skilanglauf und leichtathletische Laufdisziplinen über 400m Länge (vgl. TAYLOR 1987, 145).

${ }^{34} \mathrm{Vgl}$. Anm. 27 auf Seite 30.

${ }^{35}$ In der Regel Leistungsabfälle.
} 
FAlK/BAR-Eli 1995, 115f). Auch KleIne und SchwarZer konnten in ihrer Meta-Analyse 1991 einen deutlich engeren Zusammenhang von Angst und Leistung bei Anfängern bzw. unerfahrenen Sportlern nachweisen als bei „Experten“36 (vgl. KLEINE/SCHWARZER 1991, 16). KRANE und WILLIAMS begründeten ebenso die in verschiedenen Untersuchungen gemessenen Leistungsunterschiede bei gleicher Erregung mit der unterschiedlichen Erfahrung der Sportler (vgl. Krane/WiLliams 1987, 54f; Krane/WiLliams 1994, 210).

Andererseits existieren auch Untersuchungen, die keinerlei Unterschiede hinsichtlich der Aufregung von Anfängern und Fortgeschrittenen aufzeigen. BöCKHELER testete 1995 erfahrene und unerfahrene Fallschirmspringer auf ihre Angst und Aufgeregtheit. Er konnte keine Unterschiede zwischen Anfängern und Experten feststellen (vgl. BöcKHELER 1995, 90). Der unterschiedliche Verlauf physiologischer Messgrößen, der von FENZ und EPSTEIN berichtet wurde, ist heute selbst in Versuchen mit Erstspringern und Fallschirmspringern mit mehreren Tausend Absprüngen anscheinend nicht mehr reproduzierbar (vgl. SCHIEDEK 2000, 10; Rотн et al. 1996, 71). Dies mag natürlich auch eine spezielle Eigenart des Fallschirmspringens sein - scheinbar gilt hier aber der Zusammenhang von Angst und Leistung in Abhängigkeit des Leistungsniveaus nur eingeschränkt.

\subsubsection{Geschlecht}

Das Geschlecht wird in der Literatur als weitere mögliche Variable angeführt, die den AngstLeistungs-Zusammenhang beeinflusst. So sollen Frauen höhere kognitive und somatische Angstwerte sowie eine niedrigere Selbstwirksamkeitsüberzeugung als Männer haben. Ein für eine maximale Leistung optimaler Erregungszustand wäre also bei Frauen bei anderen Werten als bei Männern zu suchen (vgl. KRANE/WiLliams 1994, 204). Sowohl TAYLOR (1987) als auch KranE und WiLLIAMS (1994) und GraSSO (2000) konnten diese höheren Angstwerte bei Frauen experimentell bestätigen (vgl. TAYLOR 1987, 151f; KRANE/WILLIAMS 1994, 209f; Grasso 2000, 16f). NотевоOM et al. (2001) stellte zusätzlich bei Frauen eine frühere Leistungsverschlechterung bei erhöhten Angstwerten im Vergleich mit Männern fest (vgl. Noteboom et al. 2001, 2100). Auch KLEINE und SchwARZER erhielten in ihrer Meta-Analyse

\footnotetext{
${ }^{36}$ Schul- und Freizeitsportgruppen wurden im niedrigen Leistungsniveau eingestuft, einfache Wettkampfmannschaften im mittleren und Sportler oder Mannschaften aus höheren Ligen im höchsten Leistungsniveau (vgl. KLEINE/SCHWARZER 1991, 15).
} 
im Jahr 1991 einen engeren Angst-Leistungs-Zusammenhang bei Frauen (vgl. KLEINE/ SCHWARZER 1991, 15).

In der Untersuchung von GRASSO wurden weiterhin signifikant höhere Werte der Selbstwirksamkeit bei Männern, sowohl vor als auch kurz nach einer Wettkampfsituation, festgestellt (vgl. GRASSO 2000, 16f).

Um voreiligen Schlüssen und Aussagen vorzubeugen, ist allerdings zu beachten, dass für diese Geschlechtsunterschiede auch andere Gründe verantwortlich sein können: So entstammten in allen betrachteten Untersuchungen die Männer und Frauen naturgemäß unterschiedlichen Leistungsniveaus.

Weiterhin ist es gut möglich, dass Frauen offener bezüglich ihrer Angst als Männer sind. So werden Frauen potentiell mehr Angst und weniger Selbstvertrauen zugeben als Männer, zu deren Geschlechterrolle es anscheinend gehört, auf jeden Fall Stärke zu zeigen und keine Schwächen zuzugeben (vgl. Krane/WiLliams 1994, 210; Grasso 2000, 19). Dadurch kann es wieder zu einer Verfälschung der angegebenen Angstwerte ${ }^{37}$ kommen, obwohl womöglich bei beiden Geschlechtern eine gleich starke Angstreaktion auftritt.

Für dieses Argument spricht, dass in einer Untersuchung von KUBZANSKY und STEWART (1999) keine Unterschiede hinsichtlich der selbst beobachteten Zustandsangst von Männern und Frauen feststellbar waren. Bei externen Beobachtungen wurden den Frauen jedoch höhere Werte der Zustandsangst zugewiesen - KUBZANSKY und STEWART vermuten, dass die Beobachter dabei aufgrund der typischen Rollenzuweisungen geneigt waren, Frauen höhere Angstwerte zuzuordnen (vgl. KUBZANSKY/STEWART 1999, 93f). Es ist allerdings zu beachten, dass diese Untersuchung sich nicht auf Angst und sportliche Leistung bezieht ${ }^{38}$.

\subsubsection{Angstbewältigungsstile}

Wie bereits in Abschnitt 1.3.3 auf Seite 15 erläutert, können sich favorisierte Angstbewältigungsstrategien, die über den Coping-Stil einer Person ausgewählt werden, ebenfalls leistungsentscheidend auswirken. GIACOBBI und WEINBERG führen an, dass es den Anschein

\footnotetext{
37 Ähnlich wie bereits bei Hochängstlichen in Abschnitt 3.2.1.1 auf Seite 35 beschrieben.

${ }^{38}$ In der Untersuchung wurde die Angst von Musikern während des Vorspielens vor Publikum und bewertenden Dirigenten gemessen (vgl. KUBZANSKY/STEWART 1999, 82).
} 
hat, als ob der Coping-Stile eines Individuums relativ stabil ist ${ }^{39}$ (vgl. GIACOBBI/WeINBERG 2000, 47f). Daher werden im Folgenden Angstbewältigungsstile als hinreichend stabiles Element angesehen und als die Persönlichkeit beschreibenden Faktor gedeutet.

Eine leistungsbestimmende Funktion kommt den Coping-Stilen dabei hauptsächlich hinsichtlich ihrer Effizienz im Erreichen eines wünschenswerten Angst- bzw. Erregungsniveaus zu: Angst und Angstbewältigung sind nicht voneinander trennbar (vgl. Abschnitt 1.3 auf Seite 13).

\subsubsection{Aufgabenbezogene Größen}

OXENDINE führte 1970 erstmals eine Differenzierung des Angst-Leistungs-Zusammenhangs bezüglich aufgabenbezogener Größen bzw. Sportartenmerkmale ein. So soll das optimale Erregungsniveau von der Art der motorischen Anforderung ${ }^{40}$, der Aufgabenschwierigkeit und der Zeitdauer der Beanspruchung abhängen. Weiterhin werden Unterschiede zwischen den Individual- und den Mannschaftssportarten vorausgesagt (OXENDINE 1970 zit. nach KLEINE/SCHWARZER 1991, 16).

\subsubsection{Art der Anforderung}

In seiner weitergehenden Differenzierung stellte OXENDINE (1970) folgende Generalisierung des Angst-Leistungs-Zusammenhangs in Abhängigkeit der unterschiedlichen Anforderungen auf:

1. Ein hoher Grad an Erregung ist für eine optimale Leistung bei koordinativ weniger anspruchsvollen aeroben Aktivitäten mit den Anforderungen von allgemeiner Kraft, Ausdauer und Schnelligkeit nötig.

2. Ein hoher Grad an Erregung verringert die Leistung in Situationen, die eine komplexe Anforderung $^{41}$, feinmotorische bzw. anaeroben Beanspruchungen, Koordination, Gleichgewicht und allgemeine Konzentration beinhalten.

\footnotetext{
${ }^{39}$ Von einigen wenigen situationsspezifischen Ausnahmen abgesehen.

${ }^{40}$ Also gewissermaßen von der Charakterisierung grob-/feinmotorisch kognitiv hoch/niedrig beanspruchend.

${ }^{41}$ Bei der Ermittlung der Komplexität einer Aufgabe ist natürlich zu beachten, dass die Komplexität eine relative Größe ist, die von Anfängern und Experten bzw. Trainierten und Untrainierten stets unterschiedlich erlebt wird (vgl. ZIMMERMANN 2001, 81).
} 
3. Ein leicht über dem Durchschnitt liegender Erregungsgrad ist bei allen motorischen Anforderungen mit einer besseren Leistung verknüpft als ein normaler oder unter dem normalen Niveau liegender Erregungszustand (vgl. WEINBERG 1989, 104f; TAYLOR 1987, 141f).

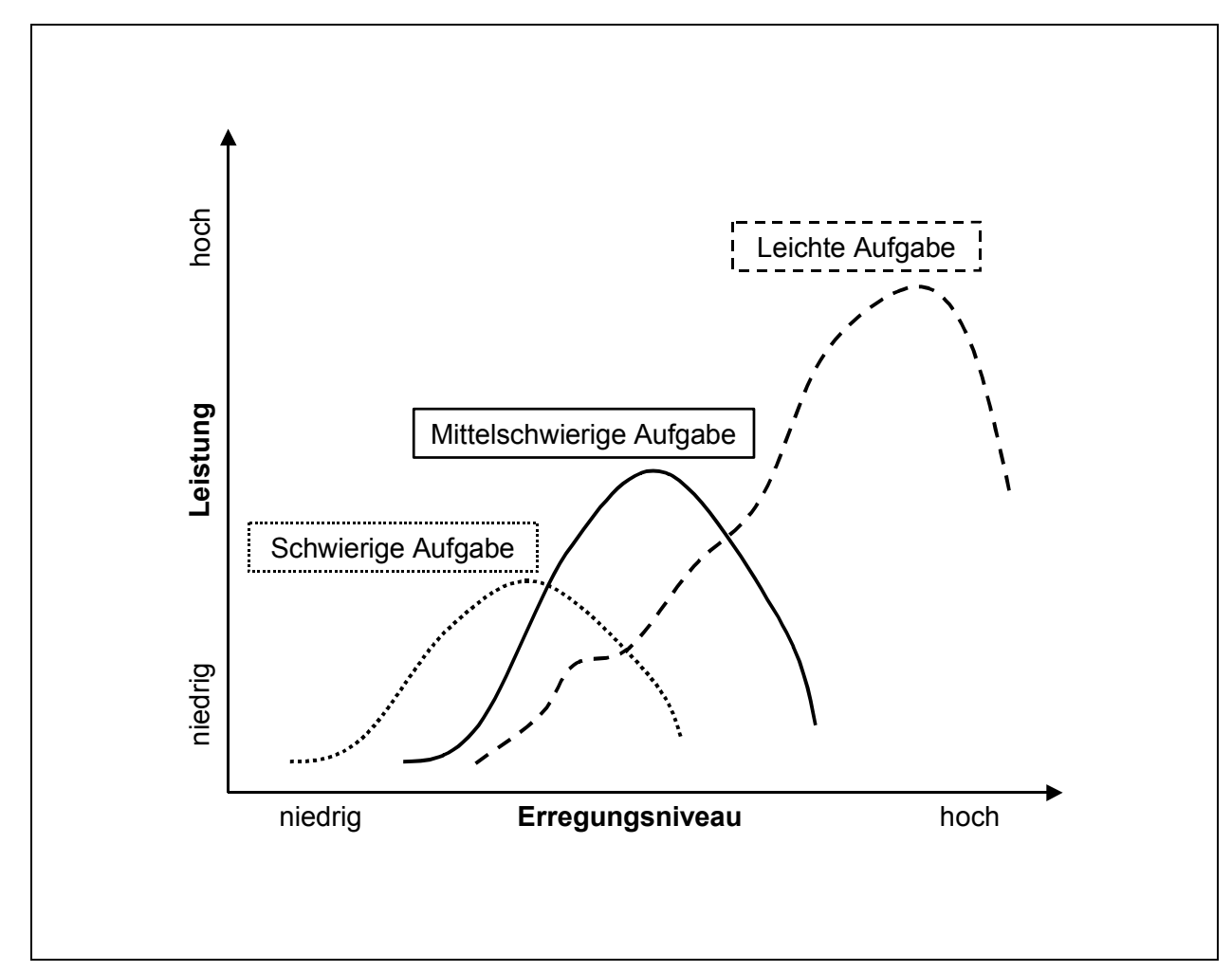

Abbildung 8: Aktivierung, Aufgabenschwierigkeit und Leistung (vgl. Zimmermann 2001, 79).

Diese Aussagen wurden nun erweitert und verifiziert ${ }^{42}$. Für jede einzelne Sportart lässt sich ein - je nach Anforderungscharakter verschiedener - optimaler Erregungszustand definieren. Tabelle 3 zeigt diese Einteilung der Sportarten anhand ihres optimalen Erregungszustandes in fünf verschiedene Gruppen: Koordinativ weniger anspruchsvolle und rein kraftspezifische Sportarten wie Gewichtheben, American Football und einige Sprintstrecken sollen ihr optimales Erregungsniveau bei der höchsten Erregung haben. Demgegenüber stehen z.B. Eiskunstlauf, Golf und Bogenschießen - hier ist die beste Leistung mit einer sehr niedrigen Erregung oder Angst verknüpft (vgl. WEINBERG 1989, 105; ClARKSON 1999, 22; ZIMMERMANN 2001, 85; BIEDERMANN 2002, 105).

\footnotetext{
${ }^{42}$ Und zwar von OXENDINE selbst, sowie auch von KLAVORA 1975 und WEINBERG und GENUCHI 1980 (vgl. WEINBERG 1989, 105).
} 
Tabelle 3: Optimale Erregungszustände für verschiedene Sportarten.

\begin{tabular}{|c|c|}
\hline Erregungsgrad & Sportarten \\
\hline 5 (extreme Aufregung) & $\begin{array}{ll}\text { - } & \text { American Football: Blocken und Tackling } \\
\text { - } & \text { Sprintstrecken zwischen } 200 \mathrm{~m} \text { und } 400 \mathrm{~m} \\
\text { - } & \text { Sit ups } \\
\text { - } & \text { Gewichtheben }\end{array}$ \\
\hline 4 (etwas aufgeregt) & $\begin{array}{ll}\text { - } & \text { Weitsprung } \\
\text { - } & 100 \mathrm{~m} \text { Sprint } \\
\text { - } & \text { Schwimmen } \\
\text { - } & \text { Skilanglauf } \\
\text { - } & \text { Judo } \\
\text { - } & \text { Ringen }\end{array}$ \\
\hline 3 (erregt, aber nicht aufgeregt) & $\begin{array}{l}\text { - } \text { Basketball } \\
\text { - } \text { Boxen } \\
\text { - Hochsprung } \\
\text { - Turnen und Gymnastik } \\
\end{array}$ \\
\hline 2 (etwas erregt) & $\begin{array}{l}\text { - Tennis } \\
\text { - Synchronschwimmen } \\
\text { - Fechten } \\
\text { - Baseball } \\
\text { - American Football: Quarterback }\end{array}$ \\
\hline 1 (sehr leicht erregt) & $\begin{array}{l}\text { - Eiskunstlauf } \\
\text { - Golf: Putten und kurze Schläge } \\
\text { - Basketball: Freiwurf } \\
\text { - Bowling } \\
\text { - Bogenschießen }\end{array}$ \\
\hline (0 (Ruhe- oder Normalzustand) & \\
\hline
\end{tabular}

Diese sportartspezifischen Unterschiede ließen sich in einigen Untersuchungen erfolgreich nachweisen: Neben Einzeluntersuchungen, die z.B. den als optimal beschriebenen niedrigen Angstlevel beim Golfen bestätigten (WEINBERG und GENUCHI 1980, vgl. WEINBERG 1989, 105), zeigte TAYLOR 1987, dass als grobmotorisch und aerob eingeordnete Sportarten ihr Leistungsmaximum bei hohen Werten von kognitiver und somatischer Angst haben. Demgegenüber war das optimale Erregungsniveau für die feinmotorischen Sportarten eher bei niedrigen und mittleren Angstwerten zu finden (vgl. TAYLOR 1987, 150).

Andererseits erfährt dieses Modell auch immer wieder Kritik: So wird die Abhängigkeit des optimalen Erregungsniveaus allein von der Anforderung der Sportarten als viel zu einfach beschrieben. So kann die Einordnung von OXENDINE keine Unterschiede hinsichtlich Wahrnehmungs- und Aufmerksamkeitsanforderungen, kognitiver Entscheidungsprozesse und 
vieler weiterer Größen, die die Anforderungen einer Sportart beschreiben, erklären. Solche komplexen Unterschiede zwischen den Anforderungen einzelner Sportarten sind aber sicherlich vorhanden (vgl. WeInBERG 1989, 106; KRANE/WiLliams 1994, 205; KiRCHNER/ STÖBER 1994, 338).

OXENDINE's Einordnung der Sportarten in die unterschiedlichen Erregungsgruppen scheint also teilweise unzureichend: Nahezu jede Klassifizierung der aufgeführten Sportarten muss mit einem hinreichenden sportwissenschaftlichen Hintergrund hinterfragt werden. So ist Gewichtheben zwar sicherlich mit dem Bild des Kraftsports eng verknüpft, die reine Bewegung des exakten Positionieren des Gewichtschwerpunktes genau über der Schulterebene jedoch hoch koordinativ anspruchsvoll. Von der motorischen Anforderung sehr verschiedene Sportarten wie Weitsprung und Judo werden in eine Klasse eingeordnet. Als letztes Beispiel wird zwar die Sportart Baseball genannt, nicht jedoch auf unterschiedliche Anforderungen der verschiedenen Spielerpositionen und -aufgaben eingegangen.

Ein möglicher Lösungsansatz wäre hier eine präzisere Klassifikation der betrachteten Sportarten hinsichtlich ihrer Anforderungsprofile. KIRCHNER und STÖBER (1994) schlagen hierzu vor, auf drei Ebenen zu agieren: Zunächst wird die Sportart einer Zielklasse bezüglich des einzulösenden Handlungsziels ${ }^{43}$ zugeordnet. Anschließend werden Bedingungsklassen identifiziert, die vordergründig Einfluss auf die motorischen Regulationsprozesse nehmen. Solche Bedingungsklassen sind etwa objektive Gesetzmäßigkeiten (Umgebungsbedingungen, wirkende Kräfte), Kooperationsanforderungen (zeitliche Abstimmungen von Einzelleistungen, Summation von Kraftwirkungen) oder Handlungsspielräume im Sinne einer möglichen Bewegungsvariation. Der letzte Schritt ist dann erst die Betrachtung der spezifischen Bewegungsanforderungen (vgl. KIRCHNER/STÖBER 1994, 342).

Weiterhin tauchen in Untersuchungen auch immer wieder „Ausreißer“ aus dem Modell von OXENDINE auf (vgl. KRANE/WiLliams 1994, 205), oder die beobachteten Unterschiede zwischen den Sportarten sind zwar erkennbar, aber in ihrer statistischen Signifikanz hinterfragbar (vgl. TAYLOR 1987, 151). Auch die Meta-Analyse von KLEINE und SCHWARZER unterstützt diese Zweifel: Unterschiede zwischen Kontakt- und Nicht-Kontaktsportarten

\footnotetext{
${ }^{43}$ Mögliche Zielklassen sind hier z.B. „Wege zwischen Start und Ziel zurücklegen“, „,einen Raumparameter der eigenen Bewegung größtmöglich ausprägen“, „räumliche Ziele genau treffen“ oder „Bewegungen mit Normen übereinstimmend darbieten“"(vgl. KIRCHNER/STÖBER 1994, 342).
} 
werden zwar bestätigt; hier ist in der Tat bei den Kontaktsportarten die maximale Leistung mit einer höheren Angst verknüpft ${ }^{44}$.

Bei grob- und feinmotorisch regulierten Sportarten wurden in der Meta-Analyse aber keine wesentlichen Unterschiede im Angst-Leistungs-Zusammenhang festgestellt. Unterschiede tauchten hier nur zwischen aeroben und anaeroben Sportarten auf, allerdings auch nur in der Feststellung, dass die Leistung bei anaeroben Sportarten mit wachsender Angst abnimmt. Bei aeroben Sportarten war kein Angst-Leistungs-Zusammenhang nachweisbar (vgl. KLEINE/ SCHWARZER 1991, 17).

Dieser Beweismangel mag aber auch durch das Design der Untersuchungen zu begründen sein: So wurden in den verschiedenen Sportarten stets verschiedene Sportler getestet, wodurch die beobachteten Effekte natürlich auch völlig zufällig durch interindividuelle Unterschiede zwischen den Probanden entstanden sein können - die breite Streuung der Angstwerte bei optimaler Leistung im ZOF- bzw. IZOF-Modell (Abschnitt 3.1.4 auf Seite 32) belegt, wie groß diese Unterschiede sogar innerhalb einer einzigen Sportart sein können.

Ein vorteilhafteres Testen der Thesen von OXENDINE sollte also so erfolgen, dass jeder Proband in verschiedenen Sportarten getestet wird. Dies verkompliziert natürlich eine Untersuchung in starkem Maße, denn wenn das Ergebnis nicht durch Leistungsunterschiede verfälscht werden soll, müssen alle diese Probanden gewissermaßen sportliche „Allrounder“ darstellen (vgl. KRANE/WiLLIAMS 1994, 205f).

\subsubsection{Zeitliche Anforderungsdauer}

Wenn man Unterschiede des Angst-Leistungs-Zusammenhangs zwischen aeroben und anaeroben Sportarten betrachtet, liegt es nahe, auch solche Unterschiede bei verschiedenen Anforderungsdauern einer Sportart zu suchen. So vermutete BURTON 1988, dass Angst ${ }^{45}$ bei kürzerer Aktivitätsdauer einen stärkeren Effekt auf die Leistung ausübt als bei längerer Dauer (BURTON 1988, 59).

Interessanterweise ließen sich solche Unterschiede z.B. bei leichtathletischen Disziplinen mit unterschiedlicher Anforderungsdauer aber nicht nachweisen (vgl. KRANE/WILLIAMS 1994, 217). Auch in der Meta-Analyse von KLEINE und SchwARZER sind keine Unterschiede

\footnotetext{
${ }^{44}$ Dies lässt sich z.B. mit einer höheren Angst vor Verletzungen begründen (vgl. ZIMMERMANN 2001, 82).

${ }^{45}$ Allerdings nur die somatische Angstkomponente.
} 
hinsichtlich der Dauer der physiologischen Beanspruchung festzustellen (vgl. KLEINE/ SCHWARZER 1991, 17).

\subsubsection{Individual- oder Mannschaftssport}

Nach MARTENS wird in Individualsportarten mitunter ein höheres Angstniveau vorausgesagt als bei Mannschaftssportarten. Dies mag daran liegen, dass Sportler in Mannschaften potentiell weniger besorgt sind - schließlich können sie im Versagensfall die Schuld mitunter auf Mannschaftsmitglieder abschieben. KJøRMO und HALVARI (2002) vermuten weiterhin, dass in Mannschaften eher die Persönlichkeit und gruppeninterne Prozesse ${ }^{46}$ leistungsbestimmende Faktoren sind (vgl. KJøRMO/HALVARI 2002, 964).

Solche Unterschiede hinsichtlich einer niedrigeren Angst von Mannschaftssportlern sind aber schwer nachzuweisen, denn es müsste hier gelingen, mehrere Sportarten miteinander zu vergleichen, die sowohl als Individual- als auch als Mannschaftssport ausgeübt werden. Ansonsten können die gesuchten Effekte durch die verschiedenen Anforderungen bei unterschiedlichen Sportarten wiederum beeinflusst oder „unsichtbar gemacht“ werden, sind also nicht evident.

Tatsächlich besteht kein signifikanter Nachweis, dass der Aspekt des Individual- oder Mannschaftssports unterschiedliche Angst-Leistungs-Beziehungen ergibt (vgl. ZIMMERMANN 2001, 83). Die Meta-Analyse von KLEINE und SchWARZER zeigt hier auch nur Unterschiede bei der somatischen, also von MARTENS nicht vorhergesagten, Angstkomponente: Diese zeigt in der Meta-Analyse bei Mannschaftssportarten keinen Zusammenhang mit der Leistung, bei Individualsportarten allerdings schon (vgl. KLEINE/SCHWARZER 1991, 18).

\subsection{Welches Angst-Leistungs-Modell ist das richtige?}

Alle hier aufgezählten Modelle versuchen, den Zusammenhang zwischen Angst und sportlicher Leistung hinreichend klar zu beschreiben. Für jedes Modell existieren Untersuchungen, die die Anwendung dieses Modells (bzw. der daraus entwickelten Theorie)

\footnotetext{
${ }^{46}$ Wie das gemeinsame Definieren und Evaluieren von Zwischenzielen der Gruppe - ein Prozess, der bei Erfolg rückwirkend Selbstwirksamkeit und Erregung des Einzelnen beeinflusst (vgl. KJøRMO/HALVARI 2002, 963).
} 
deutlich stützen - allerdings findet man ebenso viele Untersuchungen, die an der Gültigkeit Zweifel aufkommen lassen.

Die finale Erklärung des Zusammenhangs zwischen Angst und sportlicher Leistung steht also noch aus - der Zusammenhang zwischen Angst und sportlicher Leistung ist komplexer als erwartet (vgl. ZIMMERMANN 2001, 85).

Zusammenfassend lassen sich bei einem Vergleich der existierenden Studien zu den verschiedenen Angst-Leistungs-Modellen jedoch folgende Gemeinsamkeiten aller Modelle identifizieren:

1. Die Beziehung zwischen Angst und Leistung ist eher negativ.

2. Die kognitive Angstkomponente (Besorgtheit) steht anscheinend in einem engeren Zusammenhang mit der Leistung als die somatische Komponente der Aufgeregtheit.

3. Letzteres kann aber auch auf einen nicht-linearen Zusammenhang von Angst und Leistung hinweisen - oder auf eine Abhängigkeit der Angstkomponenten untereinander.

4. Der klare Nachweis der „besten“ Theorie, ob der Zusammenhang nun am besten durch das umgekehrt U-förmige, das multidimensionale oder durch neuere Theorien beschrieben wird, steht nach wie vor aus (vgl. KLEINE/SCHWARZER 1991, 25; DURTSCHI 2001, 9f).

5. Die Komplexität des Zusammenhangs zwischen Angst und sportlicher Leistung kann durch die Berücksichtigung weiterer Einflussgrößen womöglich vereinfacht oder besser erklärt werden (vgl. ZIMMERMANN 2001, 86).

$\mathrm{Zu}$ beachten ist, dass diese Ergebnisse den Stand der Forschung ohne die Nutzung neuer Theorien wiedergeben. Eine Betrachtung des Angst-Leistungs-Zusammenhangs im Rahmen der Nichtlinearen Dynamik mit neueren Modellen bietet die Chance, den dynamischen Prozess des Angsterlebens enger zu modellieren (vgl. LAZARUS 2000, 236). Solche „neueren“ Forschungsmethoden und -ergebnisse werden im folgenden Abschnitt zur Katastrophentheorie vorgestellt. 


\section{Katastrophentheorie}

Die thematische Komplexität der Katastrophentheorie ${ }^{47}$ erfordert eine ausführliche Betrachtung grundlegender inhaltlicher Aspekte der nichtlinearen Dynamik und Systemtheorie. Im Folgenden werden deshalb exemplarisch einige der wesentlichen Katastrophenmodelle erläutert und vereinfacht dargestellt. Für eine weiterführende anschauliche Erläuterung der Katastrophentheorie sei der interessierte Leser auf die Einführung von DUJARDIN (im Internet) verwiesen - eine mathematisch korrekte Entwicklung findet sich unter anderem in den Arbeiten von ZEEMAN (1977, 497-561) und ARNOLD (1999).

Im täglichen Wortgebrauch sind nahezu jedem die Begriffe Chaos und Katastrophentheorie bekannt. Jeder, der sich heutzutage mit populärwissenschaftlicher Literatur beschäftigt, kennt Beispiele für chaotische Systeme und vermag von dem Schlag des Schmetterlingsflügels zu berichten, der langfristig das Wettergeschehen beeinflussen kann. „Chaotische Grafiken“ aus fraktalen Strukturen sind jedem bekannt und werden z.B. für die Modellierung von Schneeflocken oder Landschaftsformen genutzt.

Diese chaotischen Effekte zeichnen sich dadurch aus, dass kleine Änderungen ${ }^{48}$ einer beobachteten Größe zunächst auch nur kleine und mit konventionellen (häufig linearen) Modellen vorhersagbare Änderungen des Systemzustandes nach sich ziehen. Plötzlich kommt es dann allerdings zu einer unerwarteten (chaotischen) Änderung, die zunächst unerklärbar scheint. Solche plötzlichen Änderungen oder Unvereinbarkeiten mit konventionellen Theorien wurden in der Vergangenheit als Messfehler interpretiert. Diese Fehler sind recht wahrscheinlich, schließlich können in der psychologischen oder soziologischen Forschung und Messung niemals Genauigkeiten erreicht werden, wie sie ein Physiker gewöhnt ist, der z.B. die Mechanik der Planetenbahnen untersucht. In der Psychologie, die konventionelle lineare

\footnotetext{
${ }^{47}$ Es existieren im Prinzip zwei Begriffe der Katastrophentheorie: Ein alter und ein neuer Begriff. Die sogenannte „alte Katastrophentheorie“ entstand im 19. Jahrhundert um CUVIER (1769-1832). Innerhalb dieser Theorie wurden geologische Abschnitte jeweils durch die völlige Vernichtung von Flora und Fauna durch Naturkatastrophen und anschließende Neuschöpfung gekennzeichnet. Diese Theorie war allerdings nicht lange haltbar. Die „neue Katastrophentheorie“ (Тном 1972), die im Folgenden ausschließlich behandelt wird, versteht den Katastrophenbegriff deutlich allgemeiner bzw. nicht negativ belastet. Sie versucht, den Begriff der Katastrophe als ,plötzliche Änderung“ mit mathematischen Modellen zu beschreiben (vgl. UlBRICHT 1994, 3f).

${ }^{48}$ Dies sind z.B. Änderungen an Modellparametern oder Anfangsbedingungen des Systems (vgl. MACK et al. 2000, 4).
} 
Modelle nutzte, konnte dieser Fehler allerdings nahezu 50\% betragen: Nur die Hälfte der vorhergesagten Phänomene lässt sich demnach außerhalb einer Laborsituation nachweisen (vgl. Guastello 1995, 2; SAunders 1986, 1f).

Um so hohe Fehler zu vermeiden, kann der Weg hin zur Nichtlinearität eine Lösung sein. Im Rahmen der nichtlinearen Dynamik ist der Zusammenhang zwischen zwei Größen nicht mehr zwingend linear bzw. proportional. Durch die Nichtlinearität (z.B. dargestellt durch ein Polynom $n$-ten Grades: $y=C_{n} x^{n}+C_{n-1} x^{n-1}+\ldots+C_{1} x+C_{0}$ ) kann eine abhängige Größe $y$ bei kleinen Änderungen der Kontrollgröße $x$ sowohl mit kleinen Änderungen (im Bereich der Extrema des Polynoms) als auch mit plötzlichen und dramatischen Änderungen (im Bereich einer maximalen ersten Ableitung des Polynoms) reagieren. Durch das Nutzen der nichtlinearen Dynamik kann es also gelingen, Fehler deutlich zu reduzieren - allerdings zum Preis einer wesentlich größeren Komplexität der Theorie, verbunden mit einer schwierigeren Auswertung von Messdaten (vgl. GUASTELLO 1995, 2).

Der große Vorteil dieser „neuen“ Herangehensweise im Rahmen der psychologischen Forschung ist allerdings, dass das betrachtete Phänomen nun als dynamischer Prozess und nicht mehr als statisches Element angesehen wird. Im Gegensatz zum traditionellen psychometrischen Vorgehen, welches zeitliche stabile Strukturen sucht und beschreibt, versucht eine Forschung im Rahmen der nichtlinearen Dynamik Größen zu identifizieren, welche die Änderung eines Systems beschreiben. Trotzdem wird parallel nach stabilen Zuständen eines solchen dynamischen Systems gesucht (vgl. LAZARUS 2000, 236; COMBS 1995, 129).

Die Katastrophentheorie stellt nun einen Spezialfall aus dem praktischen Umgang mit nichtlinearen Systemen dar, der insbesondere die Bewegung eines dynamischen Systems zwischen stabilen Zuständen ${ }^{49}$ untersucht. Die Nutzung dieser Theorie bietet sich stets dann an, wenn ein System stabile Zuständen annimmt. Dabei ist es egal, ob das System von Anfang an stabil ist, nach einer chaotischen Anfangsphase auf einen Attraktor zu bewegt oder plötzliche Änderungen zwischen zwei stationären Systemzuständen aufweist (vgl. SNELL et al. 1999, 26f; GILGEN 1995, 139; HANGES et al. 2002, 432).

\footnotetext{
${ }^{49}$ Stationäre Zustände (Fixpunkte), die von einem dynamischen (chaotischen) System nach Ablauf hinreichend langer Zeitspannen angenommen werden, nennt man auch Attraktoren (vgl. VoGEL 1997, 965f).
} 


\subsection{Vom Chaos zur Katastrophentheorie}

Ein System, das sich chaotisch verhält, wird durch das Auftauchen von Diskontinuitäten ${ }^{50}$ charakterisiert. Ein bei der Betrachtung solcher Diskontinuitäten genutzter Lösungsweg versucht, direkt die Eigenschaften dieser Unstetigkeit zu beschreiben, ohne zwingend auf die inneren Ursachen für diese Phänomene einzugehen. Beobachtet wird dieses System vielmehr als „Black Box“ - also als Modell, bei dem nur Anfangs- und Endzustand von Interesse sind. Der Zustand dieses Systems kann nun zu jeder Zeit durch die Werte von $n$ verschiedenen Variablen $\left(x_{1}, x_{2}, \ldots, x_{n}\right)$ beschrieben werden. Dabei ist $n$ eine endliche Zahl, die aber auch recht groß sein kann ${ }^{51}$. Gleichzeitig nimmt man an, dass der Zustand von $m$ unabhängigen Variablen $\left(u_{1}, u_{2}, \ldots, u_{m}\right)$ bestimmt bzw. beeinflusst wird. Die Zahl $m$ ist dabei recht klein (in der Regel nicht größer als 5), denn es werden alle unabhängigen Variablen außer Acht gelassen, die keinen Einfluss auf die Diskontinuität des Systems haben. Die $x_{i}$ werden als Zustands- oder innere Variable, die $u_{i}$ als Kontroll- oder äußere Variable des Systems bezeichnet (vgl. SAUNDERS 1986, 2f; GILGEN 1995, 140).

Die Erfahrung zeigt nun, dass nahezu jedes System, wenn man es lange genug sich selbst überlässt, nach einiger Zeit einen Gleichgewichtszustand annehmen wird. Dies ist bei einem schwingenden und allmählich zur Ruhe kommenden Pendel noch recht anschaulich, trifft aber auch auf fast alle anderen chaotischen Systeme zu: Auch hier streben die Systeme stets irgendwann einen Gleichgewichtszustand an. Aus der Physik aber ist bekannt, dass sich für solche Systeme, die einem Gleichgewichtszustand zustreben, stets eine Potentialfunktion ${ }^{52}$ finden lässt (vgl. UlBRICHT 1994, 9). Dabei werden sowohl die Gleichgewichtslagen als auch die Diskontinuitäten durch Extrema der Potentialfunktion ${ }^{53}$ gekennzeichnet. Die Anzahl der auftretenden Unstetigkeiten und die Zahl der qualitativ unterscheidbaren Fälle hängt dabei interessanterweise nicht von der Zahl der Zustandsvariablen $x_{i}$, sondern nur von der kleinen Zahl der Kontrollvariablen ab (vgl. SAUnders 1986, 4). Das heißt, dass die Zahl der für die

\footnotetext{
${ }^{50}$ Unstetigkeiten im Kurvenverlauf (Stellen, an denen man beim Zeichnen „den Stift absetzen muss“).

${ }^{51}$ Beschreibt man z.B. das Verhalten eines Doppelpendels, so ist $n=2$, denn man benötigt zur Beschreibung des Systems nur die Winkel der zwei Pendel mit der Senkrechten. Betrachtet man aber ein Modell des menschlichen Gehirns, kann $n$ in der Größenordnung von Millionen liegen (vgl. SAUNDERS 1986, 2; ULBRICHT 1994, 10).

${ }^{52}$ Diese Potentialfunktion wird dann natürlich nur von den Kontrollvariablen $u_{i}$ abhängen.

${ }^{53}$ Hierbei sind die Extrema mathematisch zu verstehen, können also sowohl Minimum, Maximum oder Sattelpunkt sein. Die erste Ableitung der Potentialfunktion ist an allen diesen Punkten gleich 0.
} 
Beschreibung eines Katastrophenmodells nötigen Variablen nicht größer als 6 (maximal 2 Zustands- und 4 Kontrollvariablen) sein kann - unabhängig davon, wie viele Zustandsvariablen zuvor identifiziert wurden.

Тном bewies 1972, dass sich für den Fall von maximal vier Kontrollvariablen insgesamt sieben verschiedene Arten von „Katastrophen“ unterscheiden lassen; in keiner der Katastrophen sind dabei mehr als zwei Zustandsvariablen betroffen ${ }^{54}$.

Die Unterscheidung der sieben Katastrophenformen richtet dabei ihr Augenmerk ausdrücklich auf die Form der Potentialfunktion (Anzahl und Art der Extrema) und nicht auf deren präzise Lage und Ausprägung (vgl. FREBER/SCHMID 1987, 16).

Тном klassifizierte die sieben elementaren Katastrophenformen anhand der höchsten auftretenden Potenz in Funktionsklassen und benannte sie nach ihrer jeweiligen charakteristischen Form (vgl. GuASTELlo 1995, 34f). Man erhält die Form der Katastrophen dann jeweils als Oberflächen bzw. Gleichgewichtsflächen, an denen die Potentialfunktion $V(x)$ extremal ist, an denen also gilt: $\frac{\delta V(x)}{\delta x}=0$ (vgl. SAUNDERS 1986, 57).

Tabelle 4: Die sieben elementaren Katastrophenformen nach THOM (vgl. FREBER/SCHMID 1987, 21; SANNS 2000, 58).

\begin{tabular}{|c|c|c|l|}
\hline Katastrophe & $\begin{array}{c}\text { Anzahl der } \\
\text { Zustands- } \\
\text { variablen }\end{array}$ & $\begin{array}{c}\text { Anzahl der } \\
\text { Kontroll- } \\
\text { variablen }\end{array}$ & $\begin{array}{l}\text { Funktionsklasse; } \\
\text { Potentialfunktion } \boldsymbol{V}(\boldsymbol{x})\end{array}$ \\
\hline Falte & 1 & 1 & $x^{3}+a x$ \\
\hline Spitze oder Kuspe & 1 & 2 & $x^{4}+a x+b x^{2}$ \\
\hline Schwalbenschwanz & 1 & 3 & $x^{5}+a x+b x^{2}+c x^{3}$ \\
\hline Schmetterling & 1 & 4 & $x^{6}+a x+b x^{2}+c x^{3}+d x^{4}$ \\
\hline $\begin{array}{c}\text { Hyperbolische } \\
\text { Umbilik }\end{array}$ & 2 & 3 & $x^{3}+y^{3}+a x+b y+c x y$ \\
\hline Elliptische Umbilik & 2 & 3 & $x^{3}-x y^{2}+a x+b y+c\left(x^{2}+y^{2}\right)$ \\
\hline Parabolische Umbilik & 2 & 4 & $x^{2} y+y^{4}+a x+b y+c x^{2}+d y^{2}$ \\
\hline
\end{tabular}

Es zeigte sich bald, dass sich diese von ТНОМ definierten Katastrophenformen sehr gut zur Beschreibung vieler Effekte eigneten, in denen plötzliche Änderungen von Zustandsgrößen

\footnotetext{
${ }^{54}$ Dies bedeutet, dass sich stets in einem solchen untersuchten System die Anzahl der Zustandsvariablen auf zwei transformieren (reduzieren) lassen wird (vgl. SAUNDERS 1986, 4).
} 
beobachtbar waren. Besonders hervorzuheben sind hierbei die Arbeiten von ZEEMAN (19721977), der zeigte, dass die Katastrophentheorien zahlreiche Effekte aus den verschiedensten Wissenschaftsbereichen zu erklären vermochte. Die Bandbreite der Anwendungen reicht hier von Physik und Technik über Medizin, Biologie, Psychologie bis hin zur Soziologie (vgl. ZeEmAn 1977, 2; ZeEmAn 1995, 6; Ulbricht 1994, 20f; GuAstello 2001, 11; LewisLANZA 2000, 60).

Durch diese Übertragung der Katastrophentheorie auf typisch nicht-physikalische bzw. nichtmathematische Forschungsbereiche gelang es ZEEMAN und anderen, die Katastrophentheorie zunehmend populär zu machen und die Stärken der Theorie zu nutzen:

- Die nichtlinearen Gleichungen der Katastrophentheorie ermöglichen es, zuvor als Fehler oder Zufälligkeiten gedeutete Messwerte zu erklären.

- Viele, wenn nicht gar alle beobachteten Unstetigkeiten bzw. Diskontinuitäten lassen sich durch eine der sieben elementaren Katastrophenformen beschreiben.

- Im direkten Vergleich eines gut angepassten nichtlinearen Systems gegenüber einem konventionellen linearen Ansatz beschreibt der nichtlineare Ansatz das beobachtete Phänomen nahezu doppelt so gut wie der lineare Ansatz (vgl. GUASTELLO 1995, 3).

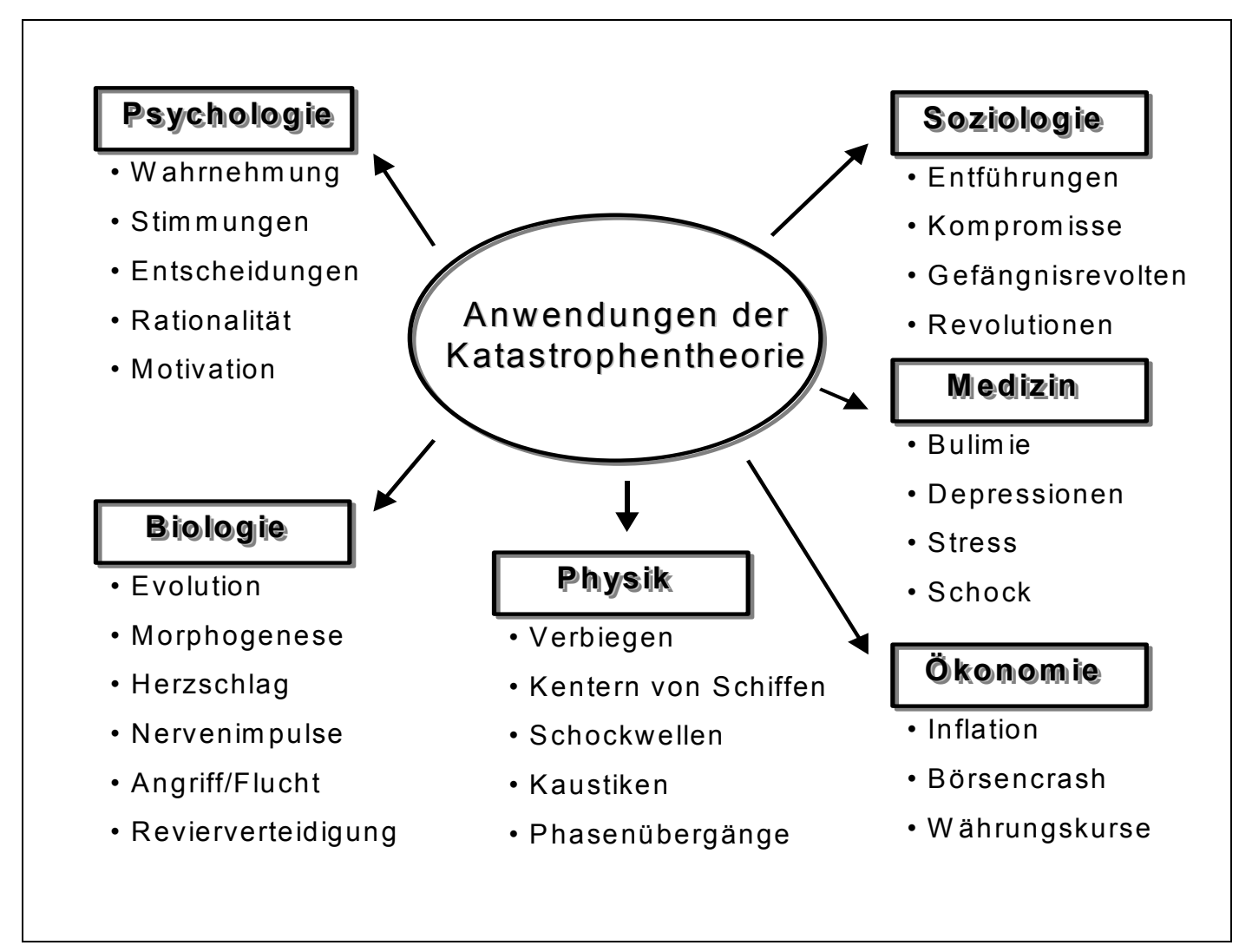

Abbildung 9: Anwendungen der Katastrophentheorie (vgl. ZEEMAN 1995, 6; GUASTELLO 2001, 19). 


\subsection{Einige elementare Katastrophenformen}

Im Folgenden werden nun einige der elementaren Katastrophenformen und ihre typischen Eigenschaften vorgestellt. Dabei gilt der Spitzen- und der Schmetterlingskatastrophe ein besonderer Augenmerk, da die Mehrzahl der erfolgreichen Anwendungen der Katastrophentheorie diese Formen nutzt (vgl. HAASE 1991, 163; UlBRICHT 1994, 14; GuASTELlo 1995, 175).

Auf eine genauere Betrachtung der umbilischen Katastrophenformen wird nicht näher eingegangen, da diese aufgrund ihrer Komplexität den Rahmen dieser Arbeit sprengen würden.

\subsubsection{Die Faltenkatastrophe}

Die Faltenkatastrophe (engl. „fold catastrophe“) ist die erste und einfachste der von THOM vorgeschlagenen Katastrophenformen. Ihre Potentialfunktion ist $V(x)=x^{3}+a x$. Dieses Potential lässt sich parametrisiert dreidimensional darstellen; man erkennt in Abbildung 10 auf Seite 54 die typische Form einer Falte, die dieser Form ihren Namen gibt.

Diese Falte entsteht im Prinzip durch das „Auseinanderfalten“ oder „Aneinanderkleben“ aller Funktionen, die die Form $z(x, y)=x^{3}+y x$ haben. Die Potentialfunktion hängt nun gewissermaßen vom „Ort“ $x$ und von der Kontrollvariable $a$ ab. Für einen konkreten Wert von $a$ erhält man eine ausgewählte Parabelform - in der Abbildung 10 auf Seite 54 zu erkennen als Schnitt entlang der $x$-z-Ebene durch die Oberfläche, die eine Parabel ergibt.

Bei einer gewissen Ausprägung des Kontrollparameters $a$ hat diese Parabel der Potentialfunktion nun ein Minimum oder eine Potentialmulde. Solch ein Minimum der Potentialfunktion stellt einen möglichen Gleichgewichtszustand des Systems dar - ein Minimum ist hierbei ein mögliches stabiles, ein Maximum ein erreichbares labiles Systemgleichgewicht. 


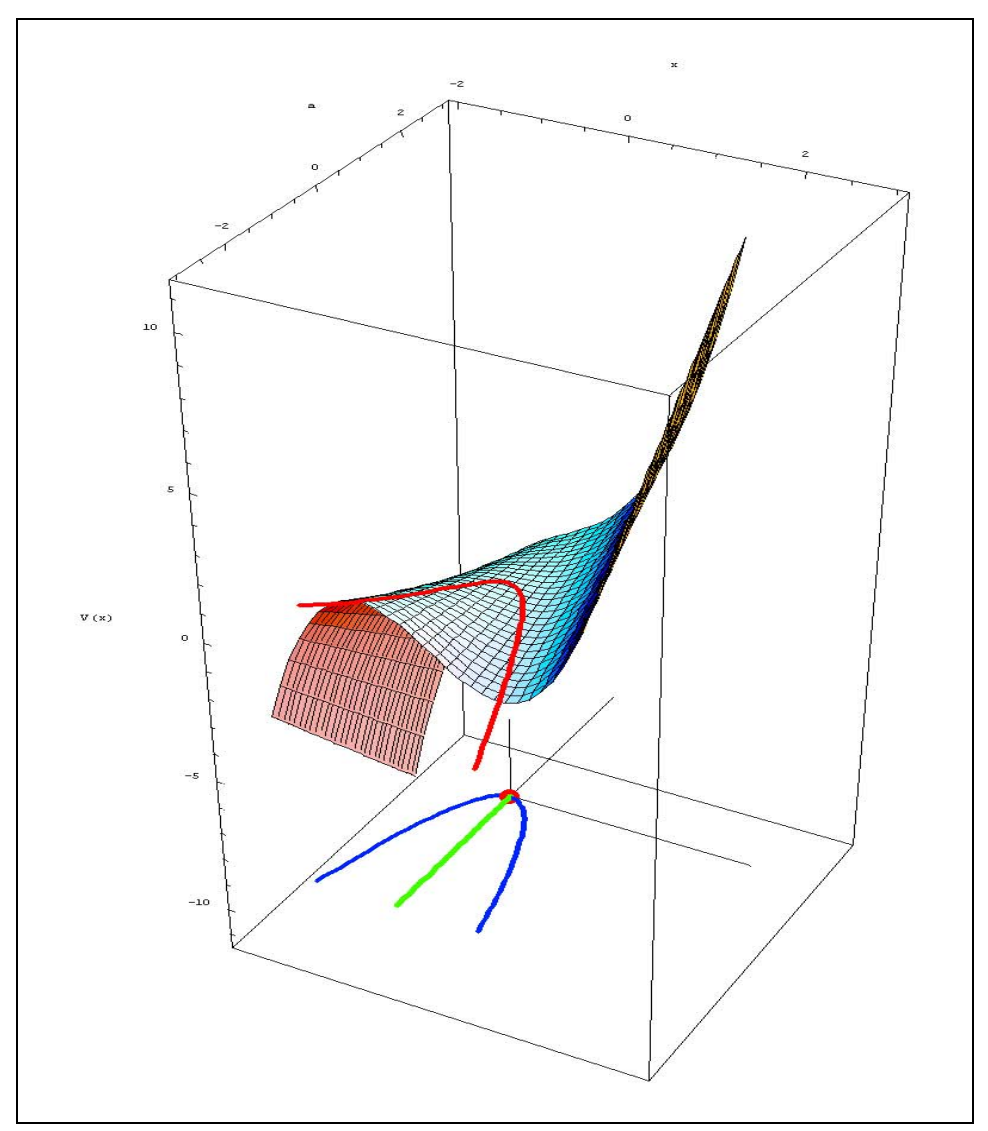

Abbildung 10: Potentialfunktion der Faltenkatastrophe (vgl. SANNS 2000, 66).

Die Katastrophenoberfläche der Faltenkatastrophe in Abhängigkeit von $x$ und $a$ wird nun durch alle die Orte bestimmt, an denen die erste Ableitung der Potentialfunktion gleich 0 ist, also an denen gilt: $x^{2}=-a$. Diese Gleichung beschreibt bei der Faltenkatastrophe eine Parabel und ist in Abbildung 10 rot eingezeichnet. Im Rahmen der Katastrophentheorie werden nun nur die Punkte der Potentialfunktion betrachtet, die mit denen der roten Parabel zusammenfallen. Man erkennt, dass diese Werte nun entweder im Bereich eines Potentialminimums $^{55}$ (für $x>0$ und $a<0$ ) oder eines Potentialmaximums (für $x<0$ und $a<0$ ) verlaufen. Das System hat also für Werte von $a$, die kleiner als Null sind, entweder einen stabilen $(x>0)$ oder einen labilen $(x<0)$ Gleichgewichtszustand. Für Werte von $a$ größer als Null hat die Gleichung $x^{2}=-a$ keine reellen Lösungen. Deshalb existieren hier keine Singularitäten oder Extremwerte und somit kein chaotisches Verhalten des Systems (vgl. SAUNDERS 1986, 58f; FREBER/SCHMID 1987, 22).

\footnotetext{
${ }^{55}$ Also gewissermaßen im Tal (der Potentialmulde) der Oberfläche in Abbildung 10.
} 
Von besonderer Bedeutung bei der Beschreibung einer Katastrophenfläche ist stets die Abhängigkeit des Systemzustands von den Kontrollgrößen. Diese Abhängigkeit wurde eben durch die Variation der Werte der Kontrollgröße $a$ exemplarisch untersucht, indem der Kontrollgröße unterschiedliche Werte zugewiesen wurden. Dabei kam es entweder zu keinen chaotischen Effekten oder zu einer Aufspaltung in zwei mögliche stabile Systemzustände. Diese Aufspaltung erkennt man in der Abbildung 10 anhand der blauen Parabel, die zeigt, bei welchen $x$-Werten in Abhängigkeit von $a$ die Potentialfunktion maximal ist, also die Gleichung $x^{2}=-a$ erfüllt ist. Diese blaue Parabel stellt folglich auch eine Projektion der typischen Katastrophenform auf die sogenannte Kontrollebene ${ }^{56}$ dar (vgl. SANNS 2000, 66).

Das Modell der Faltenkatastrophe kann zur Beschreibung der Spannung eines Gummibandes in Abhängigkeit von der Zugkraft (vgl. FreBER/SCHMID 1987, 22) oder der Stabilität einer Brücke in Abhängigkeit der Belastung (vgl. UlBRICHT 1994, 12) genutzt werden. Da die Faltenkatastrophe jedoch nur eine einzige Kontrollvariable nutzt, ist sie nicht komplex genug, um dynamische Systeme aus der Soziologie oder der Psychologie zu beschreiben (vgl. FREBER/SCHMID 1987, 22).

\subsubsection{Die Spitzenkatastrophe}

Die Spitzen-, Scheitel- oder Kuspenkatastrophe (engl. „cusp catastrophe“) hat die Potentialfunktion $V(x)=x^{4}+a x+b x^{2}$. Die Katastrophenoberfläche der Spitzenkatastrophe erhält man wiederum durch Nullsetzen der ersten Ableitung ihrer Potentialfunktion: $4 x^{3}+2 b x+a=0$. Diese Oberfläche ist dreidimensional und somit einfach graphisch darstellbar. Sie hat die typische Form einer brechenden Welle oder eines Blattes Papier, das in eine obere und eine untere Ebene gefaltet wird. Projiziert man die Kanten der Welle (in Abbildung 11 auf Seite 56 rot gezeichnet) auf die Kontrollebene, erhält man die typische Form einer Spitze (in Abbildung 11 blau dargestellt), die der Katastrophe ihren Namen gibt (vgl. ULBRICHT 1994, 15; HANGES et al. 2002, 445f).

\footnotetext{
${ }^{56}$ Also auf die Ebene, in der alle Kontrollgrößen liegen.
} 


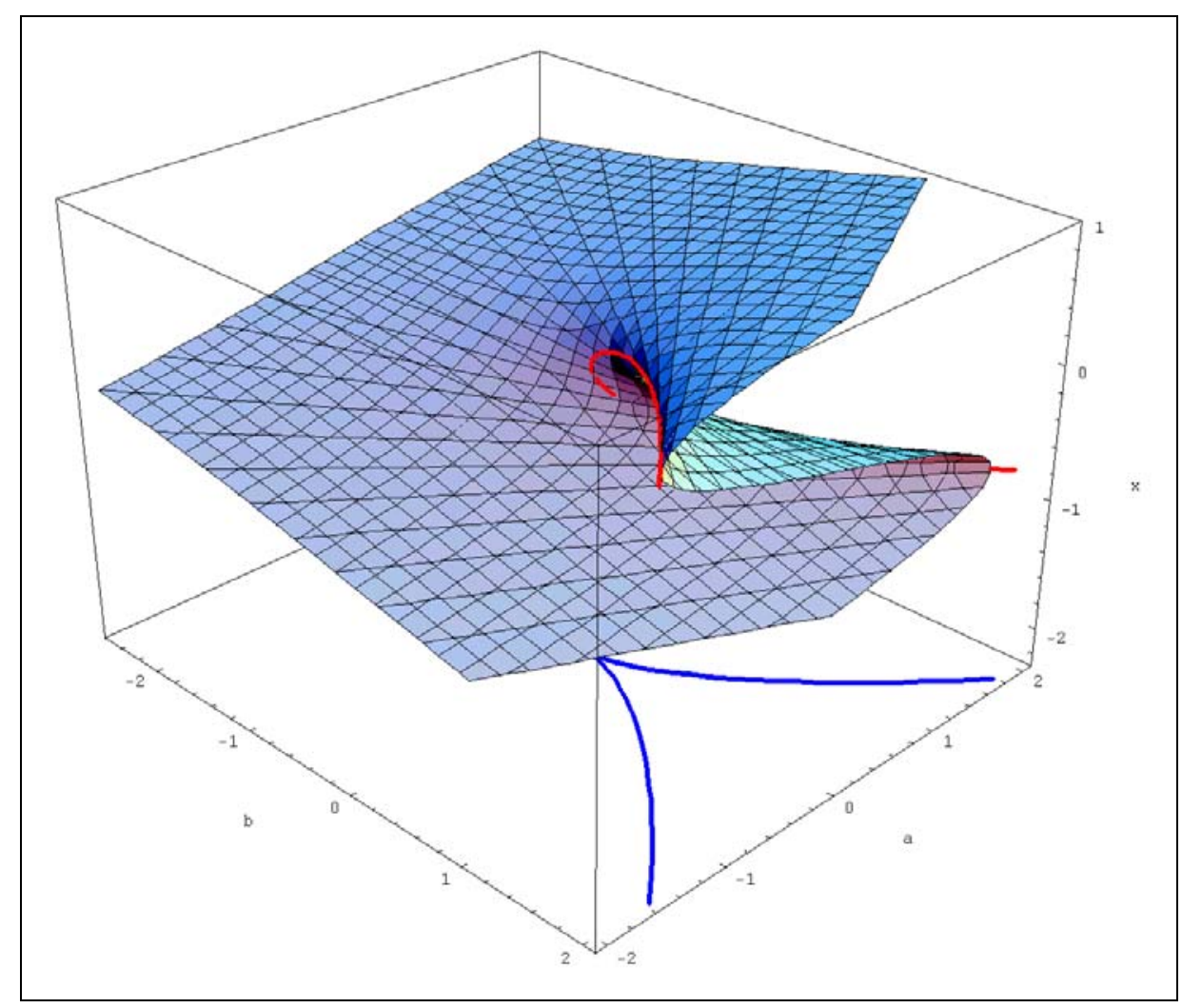

Abbildung 11: Spitzenkatastrophe (vgl. SANNS 2000, 73).

Betrachtet man nun wieder die Form der Katastrophenoberfläche in Abhängigkeit von den Kontrollvariablen $a$ und $b$, stellt man fest, dass für einen festgehaltenen negativen Wert der Variable $b$ ein ,ausgeschnittener“ Kurvenverlauf für verschiedene $a$ stets „glatt“ und stetig ist. Hier verhält sich die Kurve in Abhängigkeit von $a$ also „,normal“. Für einen festgehaltenen positiven Wert von $b$ werden dagegen unstetige Änderungen in $x$ hervorgerufen, es können Diskontinuitäten oder Sprünge ${ }^{57}$ auftreten. Die Kontrollvariable $a$ nennt man daher Normalfaktor, die Variable $b$ den spaltenden Faktor (engl. ,splitting factor“) (vgl. SAUNDERS 1986, 61; FREBER/SCHMID 1987, 23; HANGES et al. 2002, 445f).

Bewegt man sich nun in der Kontrollebene in dem Bereich, der von der blauen Spitze eingeschlossen wird (oder erreicht oder durchschreitet diese Zone von außen her), so können im Modell der Spitzenkatastrophe fünf typische Eigenschaften auftreten. In den Bereichen außerhalb der Spitze ist das Systemverhalten zu jeder Zeit stetig und mit linearen Modellen vereinbar (vgl. FREBER/SCHMID 1987, 25). Die fünf Eigenschaften der Spitzenkatastrophe im „kritischen“ Kontrollbereich sind im Folgenden aufgeführt:

\footnotetext{
${ }^{57}$ Gewissermaßen als „Absturz“ von der oberen Kante der brechenden Welle visualisierbar.
} 
1. Durch die zwei sich überschneidenden Oberflächen sind zwei (im Prinzip sogar drei) verschiedene $x$-Werte möglich. Dies lässt sich in Abbildung 11 z.B. anhand des Wertepaares $a=0$ und $b=3$ nachvollziehen. Diese Mehrdeutigkeit der $x$-Werte oder der Systemzustände bezeichnet man als Bimodalität.

2. Hält man die Variable $a$ z.B. konstant im Bereich des Ursprungs und bewegt sich in der Kontrollebene von negativen $\mathrm{zu}$ positiven Werten von $b$, so kommt es zur Divergenz: Bei positivem $b$ gabelt sich der mögliche Weg entweder zur oberen oder zur unteren Teilfläche, ohne dass dabei diskontinuierliche Sprünge auftreten. Siehe hierzu die gelbe Kurve auf der Katastrophenoberfläche in Abbildung 12 (Seite 58).

3. Der Weg von der oberen zur unteren Teilfläche erfolgt bei positiven Werten von $b$ als plötzlicher Absturz. Die Theorie definiert eine solche plötzliche Änderung als Katastrophe.

4. Es gilt weiterhin das Prinzip der Unerreichbarkeit der mittleren Faltenregion. Modelliert man die Katastrophenfläche aus einem Blatt Papier, in das eine Falte oder Welle geknickt wird, so sind nur die Punkte auf der Oberseite in der Natur oder dem Experiment erreichbar. Die „Unterseite“ des Blattes, die im Bereich der brechenden Welle als möglicher dritter (instabiler) Systemzustand vorhanden zu sein scheint, ist nicht erreichbar.

5. Diese Unerreichbarkeit im Gebiet der brechenden Welle bewirkt den Effekt der Hysterese, der z.B. aus der Physik bekannt ist: Hier hinkt die Magnetisierung von Eisen stets hinter der Ursache (der Stärke des erzeugenden Magnetfeldes) her. Ähnlich erfolgt der Sprung von der oberen zur unteren Teilebene der Katastrophenfläche an einer anderen Stelle als der Weg zurück von unten nach oben (vgl. ZEEMAN 1995, 5; SAUNDERS 1986, 17; FREBER/SCHMID 1987, 25; HANGES et al. 2002, 446). Diese Hysteresekurve ist in Abbildung 12 auf der folgenden Seite rot dargestellt (vgl. SANNS 2000, 87). 


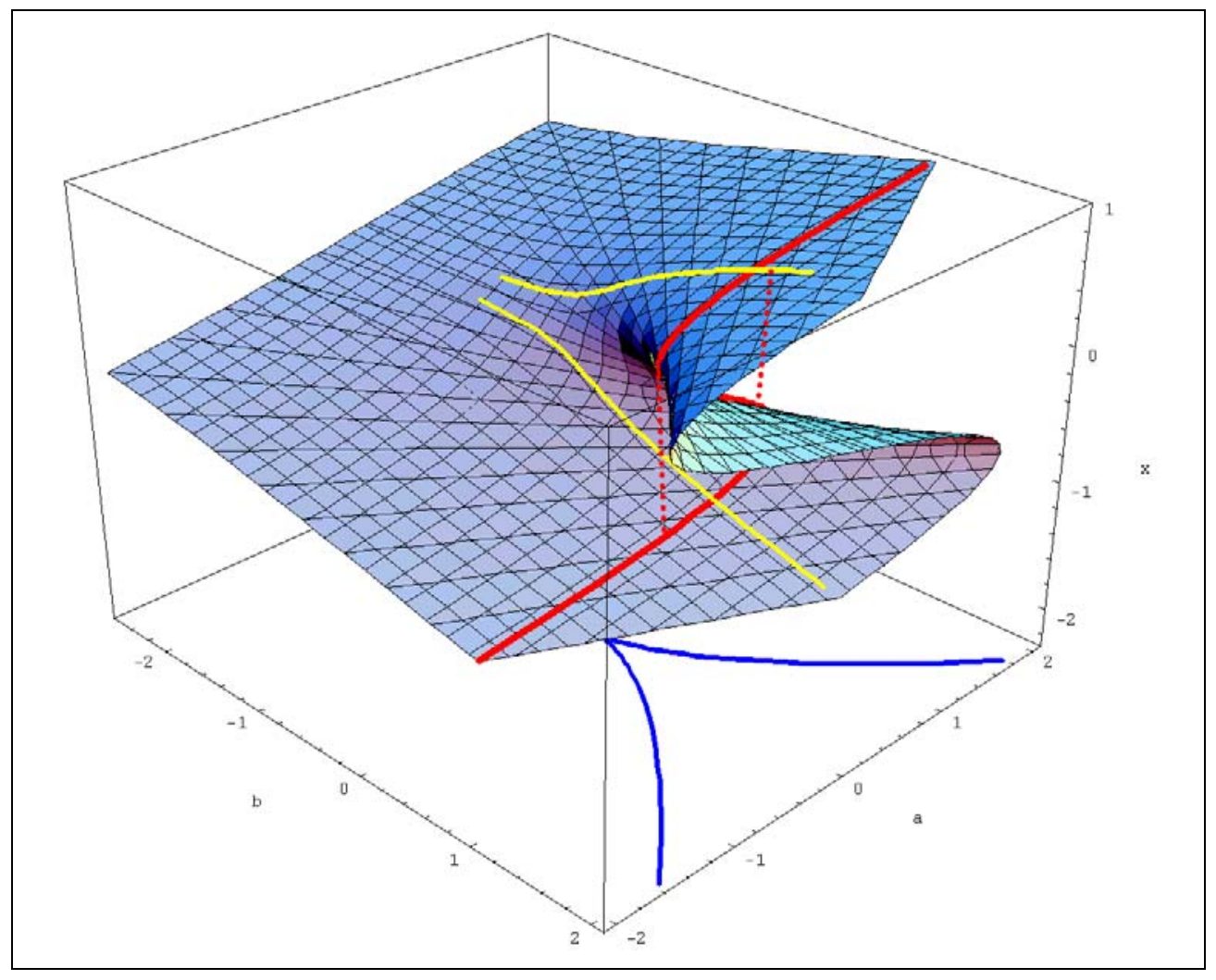

Abbildung 12: Eigenschaften der Spitzenkatastrophe (vgl. SANNS 2000, 87).

\subsubsection{Die Schmetterlingskatastrophe}

Die Schmetterlingskatastrophe (engl. „butterfly catastrophe“) kann man als Erweiterung der Spitzenkatastrophe verstehen, denn sie ist dieser in ihrer Form sehr ähnlich. Auch die Schmetterlingskatastrophe besteht im Prinzip aus zwei Oberflächen, die durch einen stetigen Übergang und durch eine Form der brechenden Welle miteinander verbunden werden. Sie hat die Potentialfunktion $V(x)=x^{6}+a x+b x^{2}+c x^{3}+d x^{4}$. Die Katastrophenoberfläche erhält man wiederum durch Nullsetzen der ersten Ableitung: $6 x^{5}+a+2 b x+3 c x^{2}+4 d x^{3}=0$.

Da diese Fläche fünfdimensional ist, lässt sie sich am besten graphisch darstellen, indem man zwei der Kontrollvariablen konstant $\operatorname{setzt}^{58}$ (in Abbildung 13 auf Seite 59 sind dies die Variablen $c$ und $d$ ).

\footnotetext{
${ }^{58}$ Durch dieses Konstantsetzen der zwei Variablen beobachtet man dreidimensionale Schnitte durch die fünfdimensionale Katastrophenfläche.
} 


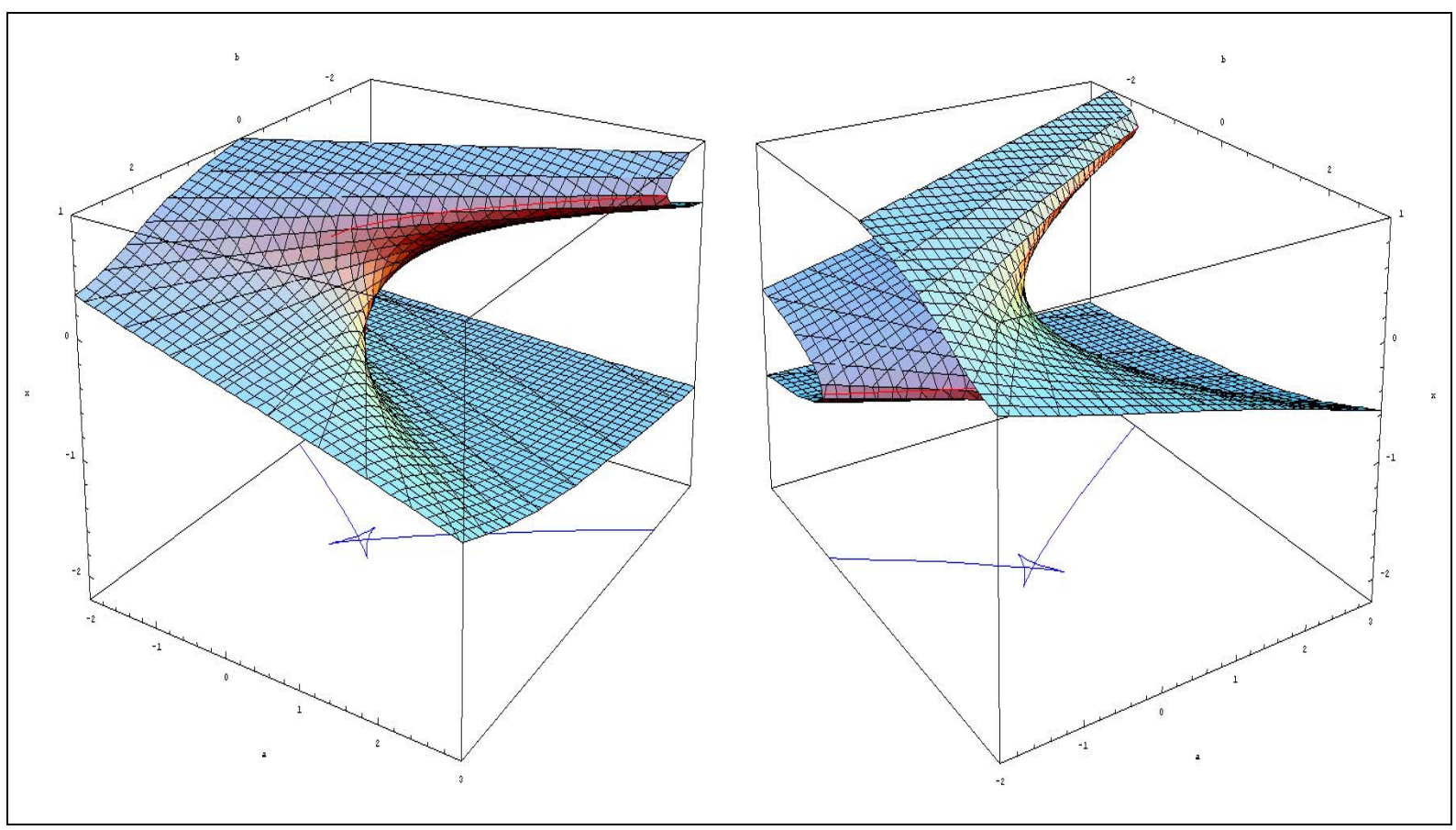

Abbildung 13: Schmetterlingskatastrophe mit Verschiebungsfaktor $c=0$ und Schmetterlingsfaktor $d=-1$ aus zwei verschiedenen Perspektiven (vgl. SANNS 2000,112).

Die Schmetterlingskatastrophe hat zusätzlich zum Normalfaktor $a$ und dem aufspaltenden Faktor $b$ mit ihren bekannten Eigenarten zwei weitere Kontrollvariablen $c$ und $d$. Die Variable $c$ bezeichnet man dabei als Verschiebungsfaktor: Ist $c<0$, so wird die Position der brechenden Welle nach links verschoben, für positive Werte von $c$ nach rechts.

Bei bestimmten Werten der Kontrollvariablen existiert bei der Schmetterlingskatastrophe ein dritter möglicher labiler Gleichgewichtszustand - gewissermaßen als Kompromiss zwischen den beiden erlaubten Zuständen der Spitzenkatastrophe. Dieser dritte Zustand taucht auf, sobald die Variablen $c=0$ und $d<0$ sind. In diesem Fall entartet die Projektion der brechenden Welle auf die Kontrollebene von der Spitze zu einem Schmetterlingsflügel (in Abbildung 13 blau dargestellt), der dieser Katastrophenform ihren Namen gibt (vgl. ZEEMAN 1977, 30; HANGES et al. 2002, 445). Sind beide Variablen gleich 0, so reduziert sich die Schmetterlingskatastrophe auf eine Spitzenkatastrophe. Entscheidend ist hierbei die Variable $d$, die bestimmt, in welchem Maße am Ursprung eine Tasche mit einer weiteren Falte, also dem dritten Gleichgewichtszustand, wächst.

Da der Faktor $d$ somit die Erscheinungsform der Schmetterlingskatastrophe moderiert, nennt man ihn auch den Schmetterlingsfaktor (vgl. FREBER/SCHMID 1987, 28; SAUNDERS 1986, 76; HANGES et al. 2002, 445f). 
Im Rahmen der Schmetterlingskatastrophe sind die gleichen fünf Grundeigenschaften zu beobachten, die auch bereits bei der Spitzenkatastrophe beschrieben wurden: Bimodalität ${ }^{59}$, Divergenz, Katastrophe, Unerreichbarkeit und Hysterese. Allerdings sind bei der Schmetterlingskatastrophe nun drei Gleichgewichtszustände möglich, und die Nutzung von vier Kontrollvariablen ermöglicht ein besseres Anpassen von komplizierteren experimentellen Zusammenhängen (vgl. BORCHARDT 1981, 39f; GUASTELLO 1995, 37).

\subsection{Angst und Leistung aus katastrophentheoretischer Sicht}

Wie bereits in Abschnitt 3 dargestellt, existierte bislang kein generelles Modell, das den Zusammenhang zwischen Angst und sportlicher Leistung zufriedenstellend beschreibt - auch wenn das IZOF-Modell von HANIN sehr komplex ist und nur wenige Ansatzpunkte für Kritik bietet. Im Folgenden werden daher die Stärken der Katastrophentheorie zur Modellierung des Angst-Leistungs-Zusammenhangs aufgezeigt.

Einen der wesentlichen Kritikpunkte an allen im vorigen Kapitel vorgestellten Modellen zum Zusammenhang von Angst und sportlicher Leistung stellt der Verlauf der Leistung in dem Moment dar, in dem das postulierte optimale Erregungsniveau verlassen wird: So erwecken alle Modelle durch den umgekehrt U-förmigen Zusammenhang den Eindruck, dass die Leistung bei einer Überhöhung der Angst langsam ${ }^{60}$ wieder abnimmt und sich vom Wert des Maximums entfernt. Viele Untersuchungen zeigen aber, dass dies nicht der Fall ist, sondern dass die Leistung im Falle der „Übererregung“ eher plötzlich und schlagartig zusammenbricht (vgl. HARDY/PARFITT 1991, 164; HARDY 1990, 82; EDWARDS et al. 2002, 7).

Ein weiterer Schwachpunkt der konventionellen Angst-Leistungs-Modelle ist die bereits in Abschnitt 3.1.3 auf Seite 30 kritisierte unterschiedliche Leistungsveränderung bei einer Zunahme der kognitiven Angst in Untersuchungen. Hier wurden sowohl Leistungsverbesserungen als auch -verschlechterungen festgestellt, obwohl die multidimensionale Angsttheorie einen ausschließlich negativen Einfluss der kognitiven Angstkomponente auf die Leistung vorhersagt (vgl. HARDY 1997, 279; HARDY/PARFITT 1991, 173; JONES 1995, 462).

\footnotetext{
${ }^{59}$ Korrekt ausgedrückt sogar Trimodalität.

${ }^{60}$ Und, mathematisch ausgedrückt, stetig, also ohne Sprünge im Kurvenverlauf.
} 
Ferner werden von der multidimensionalen Angsttheorie zwar mehrere Angstkomponenten zugelassen, zwischen den Angstkomponenten wird aber keine Interaktion berücksichtigt. Der grundlegende Ansatz der multidimensionalen Angsttheorie von MARTENS lautet nämlich, dass die einzelnen Angstkomponenten den Angst-Leistungs-Zusammenhang additiv beeinflussen (vgl. HARDY 1996a, 72; EDWARDS/HARDY 1996, 303).

Zusätzlich ist die maximale Anzahl der in der multidimensionalen Theorie enthaltenen Größen auf nur zwei Kontrollgrößen beschränkt. Einflüsse der Selbstwirksamkeit auf die Leistung werden in den konventionellen Angst-Leistungs-Modellen zwar vorhergesagt und bestätigt, können aber nur separat ${ }^{61}$ nachgewiesen werden. Die geringe Anzahl von maximal zwei Kontrollvariablen der konventionellen Theorien erlaubt keine Aufnahme weiterer Größen wie Selbstwirksamkeit, Aufgabenschwierigkeit oder bisher noch nicht identifizierter Variablen.

Die letztgenannten drei Kritikpunkte können allerdings durch Verwenden des IZOF-Modells von HANIN entkräftet werden: Dieses Modell erlaubt Interaktionen zwischen den Angstkomponenten, ebenso wie mehrere Kontrollgrößen ${ }^{62}$. Leider aber taugt dieses Modell nur bedingt für die quantitative Vorhersage des genauen zeitlichen Leistungsverlaufs, auch wenn seine Effizienz bei der Ermittlung eines optimalen Emotionsniveaus anerkannt ist.

Zur Lösung dieser Probleme entwickelten FAZEY und HARDY 1988 ein Katastrophenmodell von Angst und sportlicher Leistung, das eine mögliche Abhängigkeit der Angstkomponenten, den unterschiedlichen Verlauf der Leistung bei verschiedenen Werten der kognitiven Zustandsangst und den Effekt des „Zusammenbrechens“ berücksichtigt.

FAZEY und HARDY wählten hierfür das Modell einer Spitzenkatastrophe mit der physiologischen Erregung als Normalfaktor $a$ und der kognitiven Angst als spaltenden Faktor $b$ (vgl. JONES 1995, 467; HARDY 1990, 88). Für den Normalfaktor wurde bewusst die physiologische Erregung ${ }^{63}$ und nicht die somatische Angstkomponente des benutzten CSAI-2 gewählt, weil die Autoren sich dadurch einen engeren Angst-Leistungs-Zusammenhang versprachen. Sie vermuteten, dass die physiologische Reaktionen eines Individuums bei einer hohen Erregung schneller dazu führen, dass kognitive Prozesse behindert oder ausgeschaltet

\footnotetext{
${ }^{61}$ Also wiederum nur ohne eine Beachtung möglicher Interaktion mit anderen Angstkomponenten.

${ }^{62} \mathrm{Vgl}$. Abschnitt 3.1.4 auf Seite 32.

${ }^{63}$ In den Untersuchungen von FAZEY und HARDY gemessen durch die Herzfrequenz (vgl. HARDY 1996b, 142; HARDY 1990, 88).
} 
werden als dies bei der somatischen Angstkomponente der Fall ist (vgl. HARDY 1996b, 142; MCNALLY 2002).

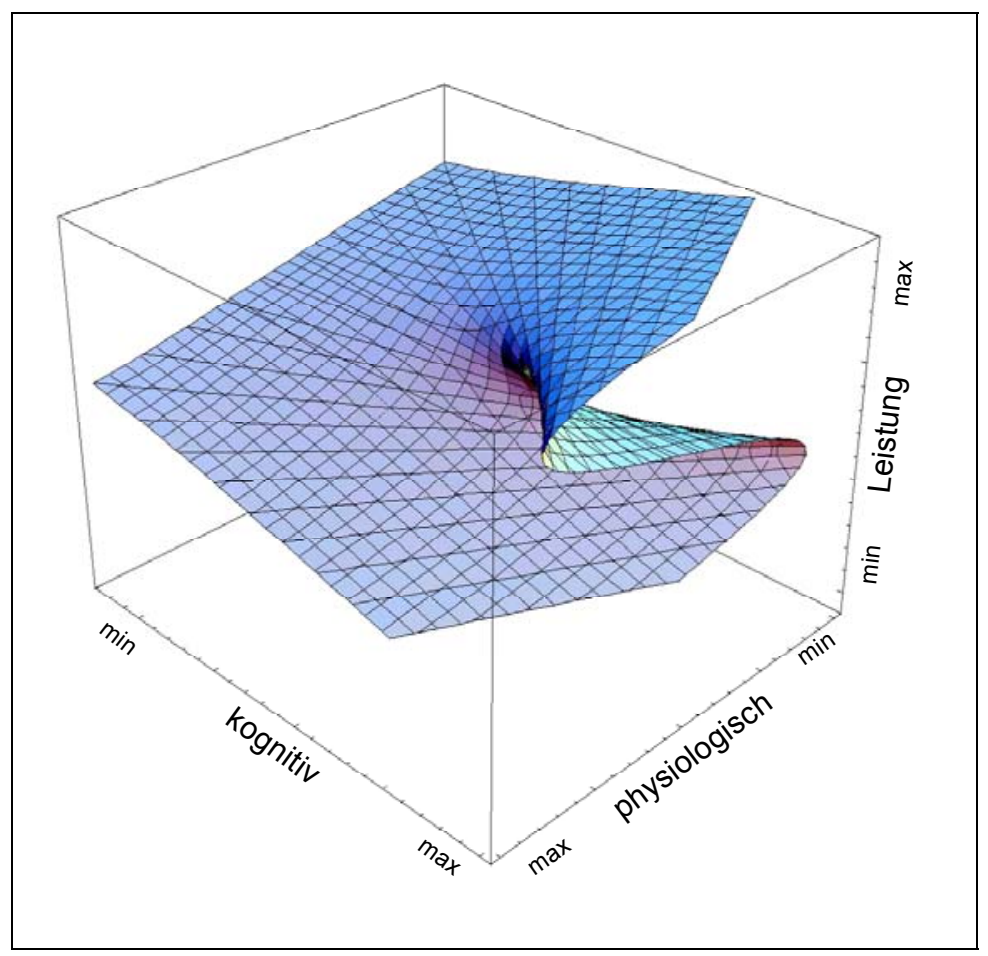

Abbildung 14: Spitzenkatastrophenmodell von Angst und Leistung (vgl. HARDY 1995, 73; HARDY 1990, 88).

Mit Hilfe des Spitzenkatastrophenmodells lassen sich nun erfolgreich alle beobachteten Effekte beschreiben:

1. Werden mittlere kognitive Angstwerte überschritten und die physiologische Erregung langsam erhöht, so wird die Leistung zunächst zunehmen und dann plötzlich zusammenbrechen. Dieser Verlauf entspricht in Abbildung 14 einem Weg auf der Leistungsoberfläche parallel zur Achse der physiologischen Erregung mit einem „Absturz“ über die Kante der brechenden Welle.

2. Ist die kognitive Angstkomponente niedrig, so ist eine stetige Abhängigkeit von Angst und sportlicher Leistung ohne Katastrophenereignisse möglich.

3. Wenn die physiologische Erregung niedrig ist, so ergibt sich eine positive lineare Beziehung zwischen kognitiver Angstkomponente und Leistung; für hohe Werte ist dieser Zusammenhang jedoch negativ linear (vgl. HARDY 1990, 88f; HARDY 1996b, 143f; HUMARA 1999). Dies entspricht in Abbildung 14 der unterschiedlichen Neigung der Leistungsoberflächen im vorderen bzw. hinteren Bereich des Koordinatensystems. 
Aus den Eigenschaften der Spitzenkatastrophe (siehe Abschnitt 4.2.2) ergeben sich allerdings noch weitere postulierte Effekte: Kommt es bei hoher kognitiver Angst zu einem Zusammenbrechen der Leistung, so tritt Hysterese auf. Um wieder auf die höhere Leistungsebene zu gelangen, muss der Sportler seine physiologische Erregung deutlich reduzieren, und zwar erheblich über den Punkt hinaus, an dem der „Absturz“ zur unteren Leistungsfläche erfolgte. Dieser Effekt der Hysterese, der von HARDY et al. in zwei Untersuchungen experimentell nachgewiesen wurde, tauchte in den konventionellen Modellen als Messfehler oder Ungenauigkeit auf, da er von diesen Modellen nicht erklärt werden konnte (vgl. KERR 1997, 100; HARDY 1996a, 72; HARDY 1996b, 146f).

Da die kognitive Angstkomponente im Spitzenkatastrophenmodell den spaltenden Faktor darstellt, ist es weiterhin sehr unwahrscheinlich, dass hohe kognitive Angstwerte gepaart mit mittleren oder durchschnittlichen Leistungswerten auftreten. Vielmehr wird hier eher ein positiver oder negativer Extremwert zu erwarten sein. Mittlere Leistungswerte sind wahrscheinlicher, wenn die kognitive Angstkomponente klein ist (vgl. HARDY 1990, 89; MCNALLY 2002).

Um die Komponente der Selbstwirksamkeitsüberzeugung auch in das Katastrophenmodell von Angst und sportlicher Leistung integrieren zu können, erweiterte HARDY das Modell 1990 zu einer Schmetterlingskatastrophe. Dabei wählte er für Normalfaktor und spaltenden Faktor wiederum die physiologische Erregung und die kognitive Angstkomponente. Für den Verschiebungsfaktor $c$, der das Eintreten von Katastropheneffekten entlang der Achse des spaltenden Faktors verschiebt, wählte HARDY die Selbstwirksamkeitsüberzeugung. Somit kann die Selbstwirksamkeit eine positive Wirkung innerhalb des Modells entfalten: Bei großen Werten der Selbstwirksamkeit können vom Sportler höhere Werte physiologischer Erregung bei gleichzeitiger kognitiver Angst toleriert werden, bevor es zum Auftreten von Katastropheneffekten kommt.

Obwohl HARDY für den Schmetterlingsfaktor $d$ keine beeinflussende Größe identifizieren konnte (und diesen Faktor als konstant annahm), eignete sich das Modell der Schmetterlingskatastrophe in seiner Untersuchung 1996 noch besser zum Beschreiben des Angst-LeistungsZusammenhangs. Ferner konnten erstmals interaktive Effekte aller drei Angstkomponenten in ein Modell integriert werden (vgl. HARDY 1996a, 83).

Wesentlicher Vorteil eines Schmetterlingskatastrophenmodells bleibt zudem, dass es mit seinen vier Kontrollvariablen hinreichend „Platz bietet“: So kann es möglich werden, alle 
Einflussgrößen des Angst-Leistungs-Zusammenhangs in ein Modell zu integrieren. Diese besondere Chance beim Verwenden der „komplizierten“ Schmetterlingskatastrophe wird von COHEN et al. (2003) und EDWARDS et al. (2002) ausdrücklich hervorgehoben (vgl. COHEN et al. 2003, 157f; EDWARDS et al. 2002, 2).

Teilweise Unterstützung erfährt das Modell der Schmetterlingskatastrophe unter anderem in einer Untersuchung von PAIN (1999). Sie konnte positive Effekte der Selbstwirksamkeitsüberzeugung auf die sportliche Leistung bestätigen, hatte jedoch erhebliche Schwierigkeiten, mit einfachen Mitteln ein Katastrophenmodell nachzuweisen. Ein einziges mit der Katastrophentheorie verträgliches Indiz in ihrer Untersuchung war die Tatsache, dass gute Leistungen mit mittlerer somatischer Erregung und geringer kognitiver Angst verknüpft waren (vgl. PAIN 1999, 1).

Weiterhin lieferten auch die neueren Befragungen von EDWARDS et al. (2002) zumindest partielle Bestätigungen der Überlegenheit eines Schmetterlingskatastrophenmodells (vgl. EDWARDS et al. 2002, 11f).

Die Erfolge des Modells der Schmetterlingskatastrophe mögen zwar Anlass zum Optimismus bieten, jedoch verweist HARDY selbst auf die Tatsache, dass die Zuordnung der Kontrollvariablen bei der Anpassung der Schmetterlingskatastrophe recht spekulativ geschah: Bereits 1988 stellten HARDY und FAZEY auch ein solches Modell vor, damals vermuteten sie jedoch die Selbstwirksamkeit als Schmetterlingsfaktor $d$ und die Aufgabenschwierigkeit als Verschiebungsfaktor $c$. Diese Zuordnung war jedoch höchst spekulativ und konnte bisher nur teilweise bestätigt werden (vgl. HARDY 1990, 99f; HARDY 1996a, 72f; EDWARDS et al. 2002, 11f).

Somit steht es noch aus, den Schmetterlingsfaktor für dieses Katastrophenmodell zu bestimmen - aufgabenspezifische Faktoren könnten hier eine Rolle spielen: Die Untersuchung von HARDY, die einen konstanten Schmetterlingsfaktor $d$ einsetzte, fand nur in einer konkreten Aufgabensituation ${ }^{64}$ statt. COHEN et al. (2003) empfehlen ebenfalls, in zukünftigen Untersuchungen die (motorischen) Aufgaben $\mathrm{zu}$ variieren und vermeintlich komplizierte Theorien vom Rang einer Schmetterlingskatastrophe zu berücksichtigen (vgl. COHEN et al. 2003, 157f).

\footnotetext{
${ }^{64}$ Nämlich den Puttschlägen mit einer Distanz von mehr als 60 Zentimetern während eines Golfturniers (vgl. HARDY 1996a, 74).
} 
Im Sinne einer bequemeren statistischen Auswertung und graphischen Darstellung mag es letztendlich geschickter sein, auf das einfachere Modell der Spitzenkatastrophe zum Beschreiben des Angst-Leistungs-Zusammenhangs zurückzugreifen. Auf diese Art und Weise lassen sich zwar nicht interaktive Effekte der Selbstwirksamkeitsüberzeugung modellieren, die Einbußen hinsichtlich einer Genauigkeit des beschreibenden Modells sind aber interessanterweise minimal. Dies stellte HARDY 1996 fest, als er im Rahmen der Anpassung der Schmetterlingskatastrophe auch ein Spitzenkatastrophenmodell überprüfte (vgl. HARDY 1996a, 82f).

\subsection{Statistische Auswertung im Rahmen der Katastrophentheorie}

Einer der Hauptkritikpunkte an dem Gebrauch von Katastrophenmodellen in Soziologie, Psychologie und anderen Anwendungsfeldern ist die komplizierte statistische Auswertung derselben. Will man mit herkömmlichen statistischen Mitteln eine Katastrophenoberfläche an Messdaten anpassen, wird die Katastropheneigenschaft der Bimodalität zum Problem. Zur Berechnung eines Fehlers ist es nicht mehr einfach möglich, zu entscheiden, ob ein Messpunkt z.B. der oberen oder unteren Gleichgewichtsfläche einer Spitzenkatastrophe zuzuordnen ist. Damit sind Anpassungsverfahren wie z.B. Regression mittels der Methode der kleinsten Fehlerquadrate oder ein $\chi^{2}$-Test nicht mehr möglich (vgl. HARDY 1996b, 144; Guastello 1995, 62; Pain 1999, 1; LANGe et al. 2001, 138). Dieses Problem der eindeutigen Wertezuordnung bei der Anpassung einer Katastrophenoberfläche wird weiterhin noch dadurch verkompliziert, dass die optimal zu Messdaten passende Katastrophenfläche gedreht, gestreckt oder verzerrt werden muss (vgl. HARDY 1996b, 149; GUASTELLO 1995, 65).

Einige Autoren versuchen, dieses Problem der komplizierten Statistik zu vermeiden, indem sie die Katastrophentheorie eher als qualitative Theorie zur allgemeinen Beschreibung und zum besseren Verständnis von plötzlichen Änderungen und anderen Katastropheneffekten nutzen. So soll die potentielle Gefahr der Willkür bei der Auswahl der Kontrollvariablen für ein quantitatives Modell minimiert werden (vgl. FREBER/SCHMID 1987, 62) - auch wenn der Preis hierfür der Verzicht auf ein tiefgehendes Analysieren des Modells ist.

Andererseits existieren sehr wohl Anpassungsverfahren, die eine quantitative Auswertung von Messdaten im Rahmen der Katastrophentheorie ermöglichen. Häufig jedoch sind diese 
Anpassungsverfahren recht kompliziert und können keine gängigen „traditionellen“ Verfahren der Statistik nutzen. Somit ist es recht mühsam, ein Katastrophenmodell mit anderen „konventionellen“ Modellen zu vergleichen, da es an akzeptierten statistischen Deskriptoren fehlt. Im Abschnitt 4.4.3 auf Seite 69 werden zwei dieser möglichen Anpassungsverfahren exemplarisch erläutert. Ferner schlägt unter anderem HARDY indirekte Verfahren vor, die ein einfacheres Testen eines Katastrophenmodells ermöglichen können.

Vorab sollte man jedoch stets prüfen, ob sich das betrachtete dynamische System rein chaotisch verhält oder sich zwischen stationären Zuständen bewegt. Dazu bietet sich eine reine Betrachtung der Zustandsvariablen des Systems an, die darüber Aufschluss geben, ob feste Systemwerte erreicht werden ${ }^{65}$. Ein weiteres Maß für die zeitliche Stabilität eines dynamischen Systems ist der Ljapunov-Exponent.

\subsubsection{Der Ljapunov-Exponent}

Der Ljapunov-Exponent $\lambda$ ist ein Maß für das chaotische Verhalten eines dynamischen Systems. Der Exponent beschreibt, wie schnell eine kleine Störung oder Variation der Anfangsbedingungen anwächst. Zur Berechnung von $\lambda$ betrachtet man den Exponenten einer angepassten Exponentialfunktion, welche den $i+1$-ten Funktions- oder Messwert aus dem $i$ ten von insgesamt $N$ Werten berechnet. Aus Gleichung 1 erhält man durch Logarithmieren den Ljapunov-Exponenten $\lambda$ - über alle Werte ist dann der Durchschnittswert des Exponenten zu bilden.

Gleichung 1: Berechnung des Ljapunov-Exponenten $\lambda$ (vgl. VOGEL 1997, 992; GUASTELLO 2002, 53).

$$
Z_{i+1}=e^{\left(\lambda \cdot Z_{i}\right)} \Leftrightarrow \lambda=\frac{\ln Z_{i+1}}{Z_{i}}
$$

Bei der Betrachtung von realen Messdaten schlägt GuASTELlo einen alternativen Berechnungsansatz nach Gleichung 2 vor, um durch zusätzliche Anpassungskonstanten $\alpha$ und $\beta$ unerklärte Varianzen der Messwerte zu berücksichtigen (vgl. GuASTELLO 2002, 55ff). Anschließend ist wiederum der Durchschnittswert für $\lambda$ über alle Werte zu berechnen.

\footnotetext{
${ }^{65}$ Bei einem Katastrophenmodell sollte man so z.B. eine Bimodalität der Zustandsvariablen zwischen zwei Werten beobachten können - bzw. eine analoge Verteilung der Messwerte (vgl. LANGE et al. 2000, 246).
} 
Gleichung 2: Alternative Form der Berechnung des Ljapunov-Exponenten $\lambda$ (vgl. GUASTELLO 2002, 55).

$$
Z_{i+1}=\alpha \cdot e^{\left(\lambda \cdot Z_{i}\right)}+\beta \Leftrightarrow \lambda=\frac{\ln \left(\frac{Z_{i+1}-\beta}{\alpha}\right)}{Z_{i}}
$$

Aus dem Durchschnittswert des Ljapunov-Exponenten $\lambda$ ist nun das Systemverhalten einfach abzulesen:

- Ist $\lambda<0$, so existiert mindestens ein Attraktor oder Fixpunkt: Das System erreicht für hinreichend große Zeiten einen stabilen Zustand. Je größer dabei der Betrag von $\lambda$, umso schneller wird der stationäre Zustand erreicht ${ }^{66}$.

- Ist $\lambda>0$, so verhält sich das System auch für große Zeiten vollständig unstabil und chaotisch (vgl. VOGEL 1997, 992; GUASTELLO 2002, 54).

Da in einem Katastrophenmodell stationäre Zustände postuliert werden, ist also bei erhobenen Messdaten ein aus der Zustandsvariablen berechneter negativer Ljapunov-Exponent zu erwarten. Andernfalls ist die Betrachtung des nichtlinearen Systems aus Sicht der Katastrophentheorie nicht zweckmäßig.

\subsubsection{Indirekte Tests eines Katastrophenmodells}

Bereits ZEEMAN versuchte, das Testen eines Katastrophenmodells ohne komplizierte Berechnungen zu ermöglichen. Er schlug vor, das Vorhandensein von mindestens zwei der fünf Eigenschaften einer Spitzenkatastrophe ${ }^{67}$ (Biomodalität, Divergenz, Katastrophe, Unerreichbarkeit und Hysterese) als hinreichenden Nachweis für ein gültiges Katastrophenmodell zu werten, falls lineare Modelle gleichzeitig versagen (vgl. ZEEMAN 1995, 5; HANGES et al. 2002, 447). HARDY greift diesen Vorschlag auf und sucht einen möglichen Beweis für das Zutreffen eines Katastrophenmodells ebenfalls in den charakteristischen Eigenschaften der Katastrophenoberflächen.

\footnotetext{
${ }^{66}$ Die Schnelligkeit gilt dabei nicht als absolute Größe, sondern in Abhängigkeit der Systemeigenschaften: Ein harmonischer gedämpfter Oszillator mit kritischer Dämpfung erreicht seinen Gleichgewichts- oder Ruhezustand von allen gedämpften Oszillatoren am schnellsten, benötigt dafür aber eine endliche Zeit (in Abhängigkeit der für die Schwingung relevanten Größen). Da er aber den Gleichgewichtszustand am schnellsten erreicht, ist der Betrag des Ljapunov-Exponenten maximal: $\lambda=-\infty$ (vgl. ELERT 2000).

${ }^{67}$ Oder einer der höherdimensionalen Katastrophenformen, denn hier treten die gleichen Effekte auf.
} 
Als erste Methode empfiehlt HARDY, den interaktiven Effekt der Angstkomponenten nachzuweisen und hierzu die Kontrollebene in vier Quadranten einzuteilen, aufgeteilt jeweils nach niedriger und hoher Angstkomponente. Die interaktiven Effekte des Katastrophenmodells sagen dann unterschiedliche Leistungsniveaus voraus, die in Tabelle 5 dargestellt sind. Diese Voraussagen können nun einer einfachen Hypothesenbildung dienen (vgl. HARDY 1996b, 144f).

Tabelle 5: Im Katastrophenmodell vorhergesagte Leistungsniveaus bei interaktiven Effekten von physiologischer Erregung und kognitiver Angstkomponente (vgl. HARDY 1996b, 145).

\begin{tabular}{|c|c|c|c|}
\hline \multicolumn{2}{|c|}{} & \multicolumn{2}{|c|}{ Physiologische Erregung } \\
\cline { 3 - 4 } & Niedrig & Hoch \\
\hline \multirow{3}{*}{ Kognitive Angstkomponente } & \multirow{2}{*}{ Niedrig } & $\begin{array}{c}\text { Mittlere } \\
\text { Leistung }\end{array}$ & $\begin{array}{c}\text { Mittlere } \\
\text { Leistung }\end{array}$ \\
\cline { 3 - 4 } & \multirow{2}{*}{ Hoch } & $\begin{array}{c}\text { Maximale } \\
\text { Leistung }\end{array}$ & $\begin{array}{c}\text { Minimale } \\
\text { Leistung }\end{array}$ \\
\hline
\end{tabular}

Diese vorhergesagten interaktiven Effekte konnten in Untersuchungen von MiLLS (1992), sowie von EDWARDS und HARDY (1996) mit konventionellen statistischen Mitteln ( $t$-Test) erfolgreich bestätigt werden (vgl. MiLls 1992, 37; EDWARDS/HARDY 1996, 302).

Als zweite Methode schlägt HARDY vor, das divergente Verhalten der kognitiven Angstkomponente für einen statistischen Nachweis eines Katastrophenmodells zu nutzen. So vermag das Katastrophenmodell zwar nicht zu erklären, warum die kognitive Angstkomponente die Leistung sowohl positiv als auch negativ beeinflussen kann. Jedoch kann das Katastrophenmodell vorhersagen, wann ein bestimmter Effekt der kognitiven Angstkomponente zu erwarten ist. So lässt sich die Hypothese bilden, dass die beste Leistung eines Sportlers signifikant erhöht und die schlechteste Leistung signifikant niedriger ist, wenn sich dieser Sportler in einem Zustand hoher kognitiver Angst befindet, als wenn der Sportler diese Leistung bei niedriger kognitiver Angst erbringt (vgl. HARDY 1996b, 146).

Mehrere Untersuchungen unterstützen diese Hypothese als Nachweis der Anwendbarkeit eines Katastrophenmodells von Angst und sportlicher Leistung (vgl. HARDY/PARFITT 1991, 173; HARDY 1996b, 146).

Als letzte einfache Möglichkeit der klassischen Hypothesenbildung nutzt HARDY den Effekt der Hysterese. So sollen im Katastrophenmodell in Momenten hoher kognitiver Angst 
Hystereseeffekte nachzuweisen sein, in Situationen mit geringer kognitiver Angst jedoch nicht.

Auch diese Hypothese ließ sich in Untersuchungen von MILLS (1992) und von HARDY et al. bestätigen (vgl. Mills 1992, 38f; HARDY/PARFitT 1991, 172; HARDY et al. 1994, 330 zit. nach HARDY 1996b, 146).

In der neueren Untersuchung von COHEN et al. (2003) konnte für die beiden letztgenannten Verfahren des Testens auf Katastropheneigenschaften jedoch kein Nachweis gefunden werden. Die Autoren, die den Zusammenhang von Angst und Leistung beim Dart-Zielwerfen aus der Sicht eines Katastrophenmodells untersuchten, vermuteten allerdings selbst, dass in dieser Untersuchung die gewählte Aufgabe nicht komplex bzw. vielschichtig genug gewählt war, um die physischen und mentalen Anforderungen realer sportlicher Gesamtbewegungen nachzubilden (vgl. COHEN et al. 2003, 155f).

\subsubsection{Numerische Näherung einer Katastrophenoberfläche}

Zur quantitativen Beschreibung eines Katastrophenmodells können verschiedene Verfahren der numerischen Näherung eines Katastrophenmodells verwendet werden: Die Methode der dynamischen Unterschiede nach GUASTELLO 1982 (engl. „,method of dynamic differences“) und die generalisierte multivariante Methode „GEMCAT“ von OLIVIA et al. 1987 (vgl. GuASTELlO 1995, 69f; HANGES et al. 2002, 448).

Beide Verfahren nutzen zunächst eine identische Herangehensweise:

1. Zunächst wird die Zustandsvariable, also die abhängige Größe und die Anzahl der stabilen Systemzustände identifiziert.

2. Hystereseeffekte und Instabilitäten werden erfasst.

3. Aus den verschiedenen Katastrophenflächen wird das Modell ausgewählt, das Punkte 1 und 2 beschreiben kann.

4. Die Kontrollvariablen und ihre Rolle (Normal- und spaltender Faktor,...) werden identifiziert.

5. Das vorgeschlagene Modell wird mit anderen bekannten Modellen, die den gesuchten Zusammenhang beschreiben, verglichen (vgl. GUASTELLO 1995, 71). 
Dazu werden Koeffizienten $\beta_{0}, \beta_{1}, \ldots, \beta_{3}$ eingeführt, mit denen Messwerte mittels linearer Regression an die Gleichung $\Delta x=\beta_{0}+\beta_{1} x^{3}+\beta_{2} b x+\beta_{3} a+\varepsilon=0$ angepasst werden.

Diese Gleichung stellt exemplarisch eine Spitzenkatastrophe dar, $\Delta x$ ist die Änderung der Zustandsvariable über ein bestimmtes Zeitintervall (vgl. GUASTELLO 1995, 66f; LANGE et al. 2001, 138).

Die freie Variation der Koeffizienten $\beta_{0}, \beta_{1}, \ldots, \beta_{3}$ ermöglicht es dabei, dass die Katastrophenoberfläche in alle Richtungen verschoben und verbogen werden kann. Auf diese Art und Weise ist es möglich, einem der Kritikpunkte von COHEN et al. (2003) zu begegnen: Diese Autoren bemängeln, dass ein qualitatives, nicht numerisch genähertes Katastrophenmodell stets den Nachteil aufweist, dass es den Ort des Auftretens von Katastropheneffekten bei mittleren Werten des Normalfaktors $a$ (physiologische Aktivierung) vorschreibt (vgl. COHEN et al. 2003, 147).

Das Verschieben und Verformen einer Katastrophenoberfläche durch quantitative numerische Näherung ermöglicht es also, ein Katastrophenmodell an situationsspezifische Besonderheiten anzupassen und auf diese Art und Weise Einzelgrößen wie Persönlichkeit, Aufgabenschwierigkeit etc. in ein individuelles Katastrophenmodell von Angst und sportlicher Leistung $\mathrm{zu}$ integrieren.

Ist nun die Aufteilung der Kontrollvariablen in Normalfaktor und spaltenden Faktor bekannt, kann man das Gütemaß ${ }^{68} r^{2}$ (das Quadrat des Korrelationskoeffizienten $r$ ) als Maß für die Relevanz des Katastrophenmodells berechnen. Dieses ist jedoch zunächst als pseudo- $r^{2}$ zu verstehen, da es nicht direkt zum Testen einer Hypothese gebraucht werden kann. Ein Vergleich mit dem Gütemaß einer herkömmlichen linearen Regression ${ }^{69}$ zwischen den Kontrollvariablen ermöglicht es dann, Aussagen über die Qualität des Katastrophenmodells (in Abhängigkeit der gewählten Variablen) zu treffen (vgl. GUASTELlo 1995, 67f; SIEVERS 1995, 606; WILLIMCZIK 1997, 76).

\footnotetext{
${ }^{68}$ Dieses Gütemaß nimmt Werte zwischen 0 (Modell beschreibt den Zusammenhang nicht) und 1 (Modell beschreibt den Zusammenhang perfekt) an; es definiert, welcher Anteil der Varianz der Zustandsvariablen durch das Modell richtig erklärt wird (ist z.B. $r^{2}=0,75$, werden 75 Prozent der Varianz durch das Modell erklärt) (vgl. WILLIMCZIK 1997, 76).

${ }^{69}$ In der Form $x=\beta_{0}+\beta_{1} a+\beta_{2} b$.
} 


\subsubsection{Methode der dynamischen Unterschiede}

Die Methode der dynamischen Unterschiede von GuASTELLO wurde unter anderem in sämtlichen Untersuchungen von HARDY et al. angewendet. Das Verfahren ist hier darauf angewiesen, dass Messdaten zu zwei nicht zu weit auseinander liegenden Zeitpunkten genommen werden. Mittels der Katastrophentheorie lässt sich nun eine Vorhersage treffen, wie der Weg von Koordinate 1 zu Koordinate 2 geschehen kann. Mit dieser Voraussage werden die realen Werte überprüft.

Grundvoraussetzung für die Anwendung dieser Methode ist jedoch das Erheben von Messwerten zu mehreren Zeiten und das Existieren einer Theorie, die definiert, welche Rolle die gemessenen Variablen im Katastrophenmodell spielen (vgl. GUASTELLO 1995, 70f; LANGE et al. 2001, 138).

\subsubsection{Das Programm GEMCAT II}

Das Programm GEMCAT II basiert auf dem generalisierten multivariablen Modell zur Anpassung von Katastrophenoberflächen von OLIVA et al. (1987). Hauptunterschied zur vorherigen Methode ist eine deutlich größere Flexibilität dieser Näherungsmethode. So werden die Kontrollvariablen des zu testenden Katastrophenmodells aus latenten, also zunächst nicht festgesetzten, Variablen mit unterschiedlichen Gewichtungen ermittelt (siehe Gleichung 3).

Gleichung 3: Latente Variablen des GEMCAT-Ansatzes für ein Spitzenkatastrophenmodell (vgl. LANGE et al. 2001, 139).

$$
\begin{aligned}
& x=\chi_{0}+\chi_{1} x_{1}+\ldots+\chi_{i} x_{i} \\
& b=\beta_{0}+\beta_{1} x_{1}+\ldots+\beta_{i} x_{i} \\
& a=\alpha_{0}+\alpha_{1} x_{1}+\ldots+\alpha_{i} x_{i}
\end{aligned}
$$

Am Beispiel des Modells der Spitzenkatastrophe, dargestellt durch die Gleichung ${ }^{70}$ : $x^{3}+b x+a=0$, optimiert der GEMCAT-Ansatz die Schätzung der einzelnen Variablengewichte durch die Minimierung der quadrierten Abweichungssumme $\phi$ von dem Idealmodell $^{71}$ der Spitzenkatastrophe über alle $N$ Messwerte (siehe Gleichung 4).

\footnotetext{
70 Zur Vereinfachung wurden die Koeffizienten vor den Variablen, in Abschnitt 4.2.2 noch vorhanden, weggelassen.

${ }^{71}$ Im Falle perfekter Anpassung eines Katastrophenmodells gilt: $\phi \approx 0$.
} 
Gleichung 4: Optimierungsgleichung des GEMCAT-Ansatzes (vgl. Lange et al. 2001, 139).

$$
\phi=\min \sum_{i=1}^{N}\left(x^{3}+a+b x\right)^{2}
$$

Der GEMCAT-Ansatz erlaubt es, dass eine gemessene Größe zu mehreren Kontrollvariablen gleichzeitig beiträgt und gibt die statistische Signifikanz der Moderation der Kontrollvariable durch die eingesetzten Messgrößen aus. So lassen sich die genäherten Parameter des gewünschten Katastrophenmodells und die Beeinflussung der Kontrollvariablen durch die Messvariablen näher untersuchen (vgl. LANGE et al. 2001, 139ff).

Dies ist für die im Rahmen dieser Arbeit durchgeführten Untersuchungen ein wesentlicher Vorteil: COHEN et al. (2003) kritisieren an den bisher verwendeten Katastrophenmodellen zum Zusammenhang von Angst und sportlicher Leistung, dass in diesen Modellen stets eine Beschränkung auf die physiologische Aktivierung oder die somatische Angstkomponente bei der Wahl des Normalfaktors $a$ stattfand (vgl. COHEN et al. 2003, 145). Durch den GEMCATAnsatz ist es nun möglich, sowohl physiologische Aktivierung als auch somatische Angstkomponente für die Anpassung des Normalfaktors $a$ zu verwenden.

Um verschiedene Katastrophenmodelle miteinander zu vergleichen und um ein Maß für die Qualität der angepassten Katastrophenoberfläche zu erhalten, gibt das Programm GEMCAT II für jedes angepasste Modell als Gütemaß einen ,pseudo-r ${ }^{26 “-W e r t ~ a u s ~}{ }^{72}$, der analog zum bereits diskutierten Gütemaß zu bewerten ist.

Im Vergleich konkurrierender Katastrophenmodelle bietet es sich weiterhin an, zu testen, bei welchem Modell die quadrierte Abweichungssumme $\phi$ signifikant geringer ist. Für einen solchen Signifikanztest schlagen LANGE et al. den WILCOXON-Test für gepaarte Stichproben vor (vgl. LANGE et al 2001, 145). Mit hinreichender Geduld lassen sich so verschiedene konkurrierende Katastrophenmodelle oder Konstellationen von Kontrollvariablen miteinander vergleichen (vgl. LANGE/HOURAN 2000, 242ff).

Das Nutzen des Programms GEMCAT II ist besonders als exploratorische Technik wertvoll, unter anderem in Situationen, in denen zwar große Datenmengen vorliegen, genauere Zusammenhänge aber stellenweise unbekannt sind (vgl. GuASTELlo 1995, 70).

\footnotetext{
${ }^{72}$ Siehe hierzu auch die Ausgaben des Programms GEMCAT II im Anhang ab Seite 175.
} 


\section{Einordnung des Fallschirmspringens}

$\mathrm{Da}$ es in der vorliegenden Arbeit um die Ermittlung des optimalen Erregungszustands für das Fallschirmspringen geht, folgt nun zunächst eine kurze Einführung in die Grundlagen des Fallschirmsports. Weil der Angst-Leistungs-Zusammenhang auch durch anforderungsspezifische Größen beeinflusst wird, werden in diesem Kapitel die sportarteigenen Anforderungen des Fallschirmspringens näher erläutert.

Aus sportwissenschaftlicher Sicht ist das Fallschirmspringen keine eigenständige sportliche Disziplin, sondern ein Oberbegriff für acht Fallschirmsportdisziplinen mit sehr unterschiedlichen Anforderungsprofilen. Die Gemeinsamkeiten dieser unterschiedlichen Disziplinen sind nur der Gebrauch des Fallschirms ${ }^{73}$ als Sportgerät und der typische funktional-technische Ablauf eines Fallschirmabsprungs (vgl. MAUSHAKE 1987, 35). Ähnliches gilt beispielsweise auch für den Oberbegriff des Skifahrens - auch hier haben Abfahrt, Langlauf, Skispringen und die weiteren sportlichen Skidisziplinen im Prinzip nur die Gemeinsamkeit der Ski als Sportgerät bei sehr unterschiedlichen Anforderungen an den Sportler.

Zur Differenzierung der unterschiedlichen Teildisziplinen ist es sinnvoll, den typischen funktionalen Ablauf eines Fallschirmsprungs in vier Phasen einzuteilen. Diese Phasen sind:

1. Der Absprung (engl. „exit“) ist der Moment, in dem der Springer das Flugzeug verlässt.

2. Der freie Fall ${ }^{74}$ ist die folgende Phase des Fallens im lufterfüllten Raum. Es wirken die beschleunigende Gewichtskraft und die bremsende Kraft der Luftreibung auf den Fallschirmspringer ein: Sind diese Kräfte gleich groß, so ändert sich die Fallgeschwindigkeit nicht $\mathrm{mehr}^{75}$. Der Freifall wird durch die Phase der Fallschirmöffnung beendet.

\footnotetext{
${ }^{73}$ Im Fallschirmsport werden heute quasi ausnahmslos brems- und steuerbare Flächenfallschirme verwendet.

${ }^{74}$ Physikalisch betrachtet findet ein freier Fall nur im Vakuum statt. Umgangssprachlich und in den meisten Veröffentlichungen zum Fallschirmspringen hat sich jedoch der Begriff des Freifalls als Bezeichnung für den Fall des Springers im lufterfüllten Raum durchgesetzt und wird daher im Folgenden ebenfalls so gebraucht.

${ }^{75}$ Mit einer „normalen“ Körperhaltung erreicht der auf dem Bauch fallende Springer so eine Geschwindigkeit von ca. 180-200km/h (vgl. SCHÄFER 1998, 53).
} 
3. Die Sinkphase am geöffneten Fallschirm, die sich aufgrund des heutzutage nahezu ausschließlichen Gebrauchs von Flächenfallschirmen auch als Flugphase mit dem Fallschirm bezeichnen lässt.

4. Die Landephase beendet den funktional-technischen Ablauf eines Fallschirmsprungs (vgl. MAushaKe 1987, 45; MÜLler 1989, 18).

Die verschiedenen Fallschirmsportdisziplinen werden im Folgenden näher beschrieben - alle diese Teildisziplinen sind vom Weltluftfahrtverband FAI (Fédération Aéronautique Internationale) offiziell anerkannte Wettbewerbssportarten, in denen Deutsche Meisterschaften, Europa- und Weltmeisterschaften ausgetragen werden.

\subsection{Die Fallschirmsportdisziplinen}

Die Übersicht der einzelnen Fallschirmsportdisziplinen erfolgt aufgeteilt nach den Phasen des Fallschirmsprungs, in denen sie ausgeführt werden. Dies sind die Sink- oder Flugphase am geöffneten Fallschirm und die Phase des Freifalls.

Die Sportarten, die am geöffneten Fallschirm ausgeübt werden, sind Zielspringen und Fallschirmformationsfliegen. Diese zwei Disziplinen werden von MÜLLER so klassifiziert, dass eher der materiell-technische Faktor leistungsbestimmend ist - es geht in beiden Disziplinen um die Steuerung des Fallschirms (vgl. MüLLER 1989, 25).

Dem gegenüber stellt MÜLLER die Sportarten, die im freien Fall ausgeübt werden. In diesen stellen technisch-koordinative Faktoren (sportartspezifische Gewandtheit, Geschicklichkeit, Gleichgewichtsgefühl, Orientierungsvermögen, Antizipation, peripheres Sehen) den Hauptteil der leistungsbestimmenden Komponenten der sportlichen Handlung dar (vgl. MüLLER 1989, 25; MAUSHAKE 1987, 35f). Zu den Disziplinen, die im freien Fall ausgeführt werden, zählen Figurenspringen, Freestyle, Skysurfen, Freeflying und das Freifallformationsspringen. Im Folgenden werden alle diese Disziplinen kurz beschrieben - das Freifallformationsspringen wird in einem gesonderten Abschnitt ausführlicher behandelt, da sich der experimentelle Teil dieser Arbeit aus Gründen der besseren Vergleichbarkeit ausschließlich mit dem Formationsspringen befasste. 


\subsubsection{Zielspringen}

Seit jeher konzentrieren sich Fallschirmspringer darauf, den gewünschten Landeplatz anzusteuern, sobald sie ihren Fallschirm geöffnet haben. Hieraus entstand die älteste und ursprüngliche Fallschirmsportdisziplin, bei der immer kleinere Landeflächen angesteuert wurden. Die Zielscheibe, auch Nullscheibe genannt, hat bei heutigen Wettkämpfen einen Durchmesser von $3 \mathrm{~cm}$ und ist damit nur drei Millimeter breiter als eine 2-Euro-Münze. Spitzensportlern gelingt es dabei regelmäßig, dieses kleine Ziel mit dem ersten Bodenkontakt zu treffen.

Für das Zielspringen werden spezielle Fallschirme verwendet, die sich durch eine sehr langsame Vorwärtsfahrt und ein deutlich schwerfälligeres Drehverhalten von gewöhnlichen Flächenfallschirmen unterscheiden (vgl. SCHÄFER 1998, 66ff; MAUSHAKE 1987, 36).

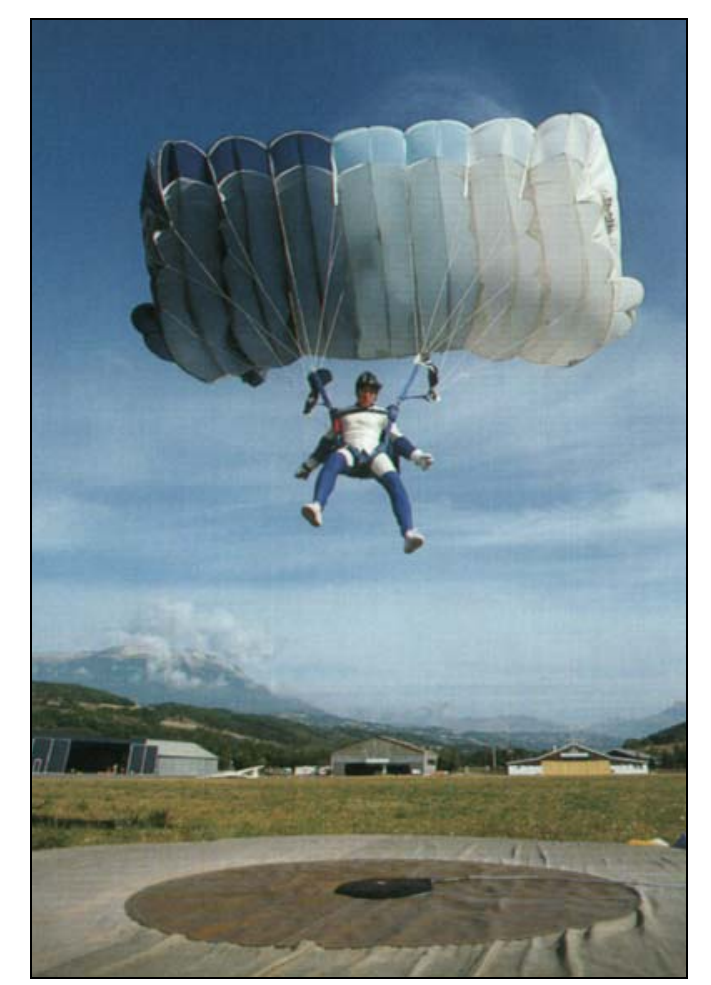

Abbildung 15: Zielsprung (in: SCHÄFER 1998, 68).

\subsubsection{Fallschirmformationsfliegen}

Das Fallschirmformationsfliegen, auch Kappenformationsspringen genannt, ist eine der jüngeren Disziplinen des Fallschirmsports. Hierfür werden zwingend Flächenfallschirme benötigt, die in der Regel mit besonderen Verstärkungen versehen sind. Es gilt nun, mehrere Fallschirme in einer gemeinsamen Formation $\mathrm{zu}$ halten. Dies erfolgt nach dem 
Zusammenfliegen durch Festhalten von Fangleinen oder Fallschirmgewebe eines in der Formation benachbarten Fallschirms. In Wettbewerben treten 4er- oder 8er-Mannschaften gegeneinander an. Hierbei müssen in einer festgelegten Zeit möglichst viele vorher festgelegte Formationen geflogen werden (vgl. SCHÄFER 1998, 68ff).

Der Weltrekord für die größte Kappenformation liegt heute bei 53 Springern (vgl. FAI im Internet).

\subsubsection{Figurenspringen}

Das Figurenspringen, das auch als Stilspringen bezeichnet wird, ist die älteste der Freifallsportarten. Diese Individualsportart bezieht sich auf die elementaren „fliegerischen“ Fähigkeiten des Sportlers. Es gilt, innerhalb der kürzesten Zeit eine Folge von zwei 360-GradDrehungen und einem Rückwärtssalto zweimal möglichst exakt und fehlerfrei zu zeigen. Mangelnde Präzision, Überdrehen oder ein Verlassen der ursprünglichen Richtungsachse, in der das Programm begann, werden mit Strafsekunden belegt. Die weltbesten Figurenspringer absolvieren das komplette Programm ${ }^{76}$ in unter sechs Sekunden. Dazu versuchen sie, eine möglichst hohe Fallgeschwindigkeit zu erreichen, da dies ein präziseres Manövrieren im Freifall ermöglicht (vgl. SCHÄFER 1998, 55f; HELLER 2002, 86).

\subsubsection{Freestyle}

Freestyle bedeutet direkt übersetzt „Freistil““ und entstand als Erweiterung des klassischen Figurenspringens. So wurde die Zahl der Elemente gegenüber dem Stilspringen durch Hinzufügen neuer Manöver ${ }^{77}$ auf über 80 erhöht. Innerhalb eines Wettkampfes werden ausgewählte Elemente in Pflicht- und Kürsprüngen zu einer Choreographie vereint, die sich im dreidimensionalen Raum abspielt. Alle Bewegungen werden durch einen mitspringenden Kameraflieger aufgezeichnet - der Kameraflieger wird dabei innerhalb des Sprunges eine Vielzahl von Positionen einnehmen, um die Bewegungen des Springers optimal in Szene zu setzen. Bewertet wird die Mannschaftsleistung von Freestyler und Kameraflieger (vgl. SCHÄFER 1998, 59f; HELLER 2002, 96ff).

\footnotetext{
${ }^{76}$ Also insgesamt vier 360-Grad-Drehungen und zwei Rückwärtssalti.

${ }^{77}$ Rotationen um alle Achsen des Körpers, Spagate, dynamische Übergänge und andere Elemente.
} 


\subsubsection{Skysurfen}

Das Skysurfen ist heutzutage nahezu jedem bereits in den Medien begegnet. Vereinfacht ausgedrückt ist Skysurfen nichts anderes als Freestyle mit einem speziell angefertigten Surfbrett unter den Füßen. Innerhalb eines Wettkampfes gilt die gleiche Aufteilung in Pflichtund Kürsprünge, die mit Choreographien gefüllt werden, wie beim Freestyle. Auch in dieser Sportart wird die Mannschaftsleistung von Skysurfer und Kameraflieger bewertet.

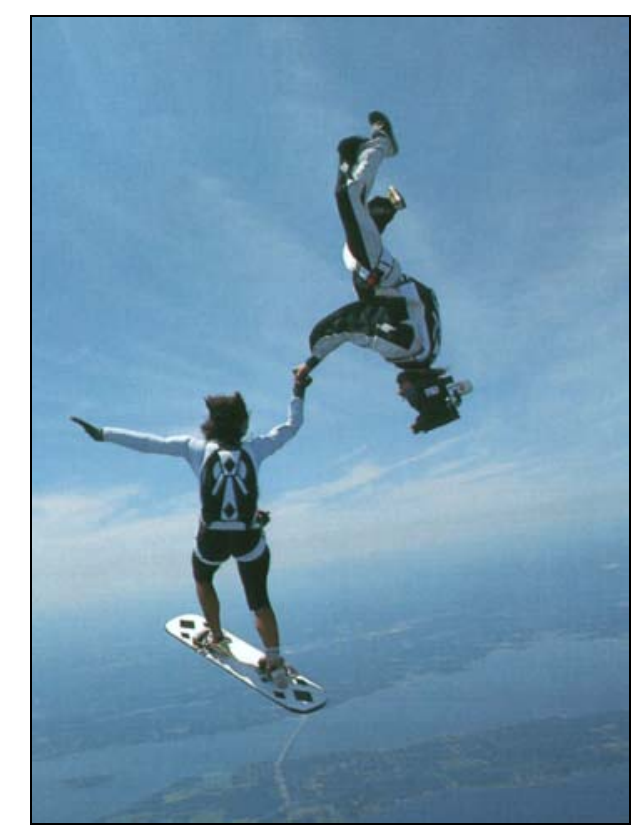

Abbildung 16: Skysurfer und Kameraflieger (in: SCHÄFER 1998, 61).

\subsubsection{Freeflying}

Das Freeflying ist die jüngste Disziplin im Fallschirmsport. Sie hat ihre Ursprünge ebenfalls im Freestyle. Innerhalb dieser Sportart müssen zwei Springer Übergänge zwischen dreidimensionalen Formationen und Bewegungen (engl. „transitions“) zeigen, die ebenfalls in Pflicht- und Kürsprüngen ausgelost werden. Grundposition beim Freeflying ist dabei das Fliegen im Kopfstand, bei dem Geschwindigkeiten über $300 \mathrm{~km} / \mathrm{h}$ möglich sind. Ein Kameraflieger hält die Bewegungen der Zweiermannschaft auf Videoband fest. Von diesem Springer werden im Rahmen des Freeflyprogramms Pflichtbewegungen oder -blickwinkel verlangt (vgl. HELLER 2002, 96ff; DFV e.V. im Internet). 


\subsection{Freifallformationsspringen}

Die Untersuchungen zum optimalen Erregungsniveau bei Fallschirmspringern im praktischen Teil dieser Arbeit wurde aus Gründen der Einheitlichkeit und der Vergleichbarkeit nur an Freifallformationsspringern durchgeführt. Daher erfolgt eine ausführlichere Beschreibung dieser Sportart und der Versuch einer Einordnung ihrer aufgabenbezogenen Größen, die den Angst-Leistungs-Zusammenhang beeinflussen.

\subsubsection{Grundgedanke des Freifallformationsspringens}

Das Freifallformationsspringen, auch kurz Formationsspringen, Relativspringen oder abgekürzt RW (engl. für „relative work“) genannt, entstand 1958 in Kanada, als die erste dokumentierte Übergabe eines Stabes im freien Fall gelang. Diese ersten Näherungsversuche von Sportlern im Freifall wurden schnell um das Bilden von Formationen durch Greifen von Armen oder Beinen anderer Springer erweitert. Langsam wurde die Zahl der beteiligten Fallschirmspringer größer. Dabei mussten zunächst die für das Formationsspringen elementaren Grundtechniken entwickelt werden: Hierzu zählen das Ausgleichen der Fallgeschwindigkeit an die der anderen Springer, gezielte Vorwärts-, Seitwärts- und Rückwärtsfahrt sowie das Drehen, ohne dabei die Position im dreidimensionalen Raum zu verlassen. Dies war allerdings eine langwierige und mühsame Entwicklung. Erst 1965 gelang die erste Achterformation, 1967 in Kalifornien der erste 10er-Stern (vgl. SCHÄFER 1998, 56).

Heute liegt der Rekord für die größte Freifallformation bei 300 Fallschirmspringern (vgl. HoEnLE 1996, 25; FAI im Internet). Das Formationsspringen hat sich inzwischen zur populärsten und am weitesten verbreiteten Disziplin im Fallschirmsport entwickelt (vgl. MAUSHAKE 1987, 37; DFV e.V. im Internet).

\subsubsection{Wettbewerbe im Formationsspringen}

Seit 1975 gibt es Weltmeisterschaften im Formationsspringen, damals aufgeteilt in 4er- und in 10er-Mannschaften. Heute finden alle Wettbewerbe in einer 4er- und einer 8er-Wertung statt. Ein Wettbewerb besteht in der Regel aus 10 Runden $^{78}$ bzw. Sprüngen, zwischen zwei aufeinanderfolgenden Runden muss der Mannschaft eine Pause von mindestens 60 Minuten

\footnotetext{
${ }^{78}$ Einschließlich Halbfinal- und Finalrunde mit jeweils reduzierter Teilnehmerzahl.
} 
gewährt werden, die Maximalzahl der pro Tag pro Mannschaft erlaubten Wertungsrunden kann dabei 6 überschreiten.

In einem Formationssprung müssen die Mannschaften in 35 Sekunden (4er) bzw. 50 Sekunden (8er) verschiedene, aus dem Wettbewerbsprogramm ausgeloste, Folgen von fünf oder sechs Formationen mit festen und freien Übergängen fliegen. Die Zeit zählt von dem Moment an, in dem der erste Springer der Mannschaft das Flugzeug verlässt.

Die Absprunghöhe beträgt hierbei im 4er 3200m und in der 8er-Disziplin 4000m über Grund.

Die Phase des Absprungs wird zu einer der zentralen Komponenten des Formationsspringens, die gesamte Mannschaft versucht, möglichst kompakt das Flugzeug zu verlassen. Spitzenteams aus der 4er-Wertung springen dabei bereits in der ersten geforderten Formation vom Flugzeug ab - in der 8er-Formation ist dies nur bei ausgewählten Formationen oder beim Springen aus großen Frachtflugzeugen mit einer Heckrampe möglich. Diese Flugzeugtypen werden aber auf Wettkämpfen nur sehr selten eingesetzt (vgl. JEFFERIES 1998a).

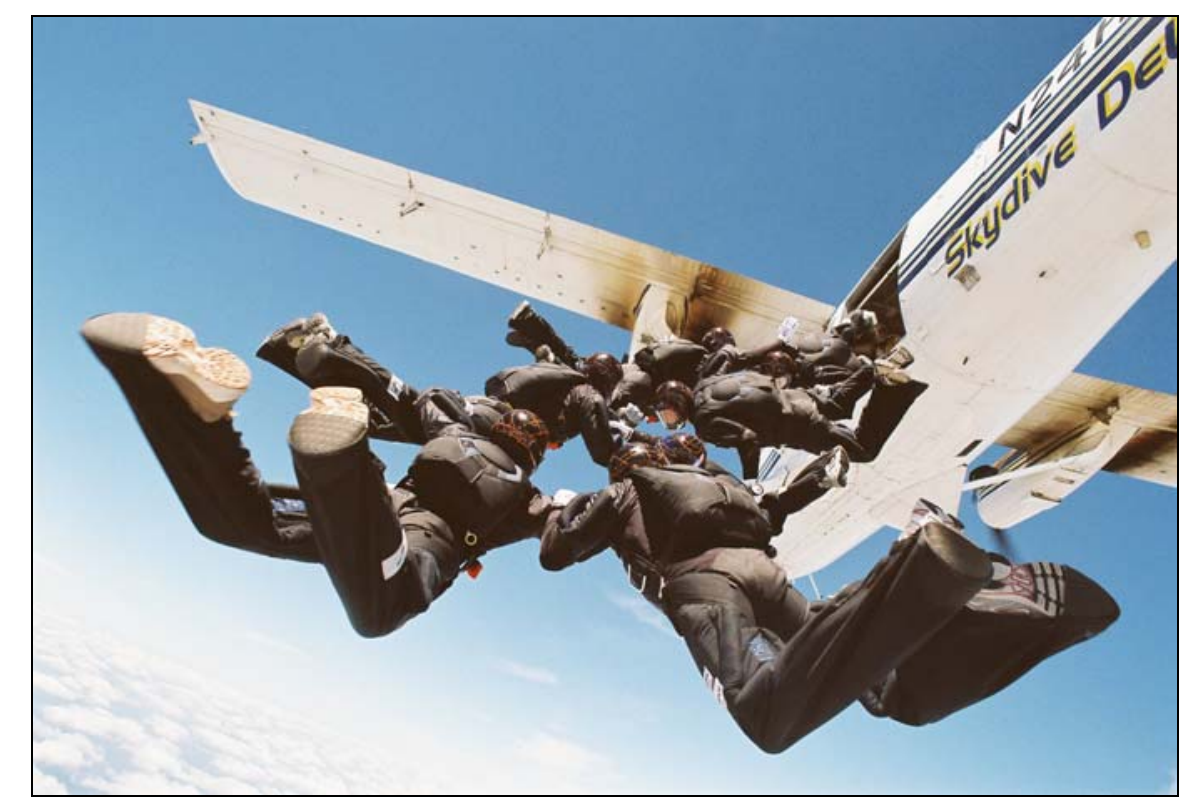

Abbildung 17: Absprungphase einer 8er-Mannschaft (Foto: Edson Pacheco).

Die zu fliegenden Folgen aus Formationen werden aus einem Programm gelost, in dem pro Disziplin 16 freie Formationen und 22 Formationen mit einem festen Übergang enthalten sind. Das vollständige Formationsprogramm der 4er- und 8er-Disziplinen findet sich im Anhang; Abbildung 18 auf der folgenden Seite zeigt einen typischen festen Übergang des 8erProgramms. 
Ein fester Übergang ${ }^{79}$ bedeutet, dass es sich hier um eine Anfangs-, eine Endformation und einen vorgeschrieben $\mathrm{Weg}^{80}$ von der einen zur anderen handelt. Demgegenüber erfolgt die Bewegung von einer freien Formation ${ }^{81} \mathrm{zu}$ der nächsten lediglich durch das komplette und gleichzeitige Lösen aller Griffe der Freifallformationsspringer (vgl. SCHÄFER 1998, 57f).

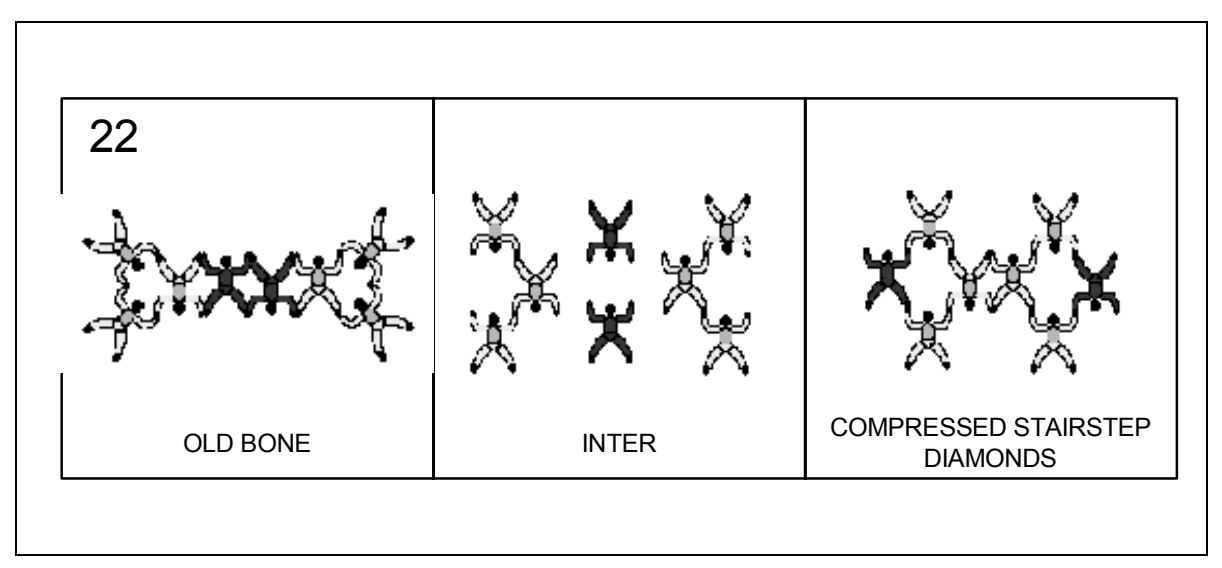

Abbildung 18: Blockübergang 22 von „Old Bone“ "zu „Compressed Stairstep Diamonds “im 8er-Programm (eigene Darstellung).

Die Bewertung eines Formationssprunges erfolgt wiederum anhand der Videoaufzeichnungen eines mitspringenden Kamerafliegers. Jede innerhalb der Arbeitszeit korrekt gezeigte Formation der ausgelosten Sequenz wird mit einem Punkt bewertet, bei Fehlern werden zwei Punkte abgezogen.

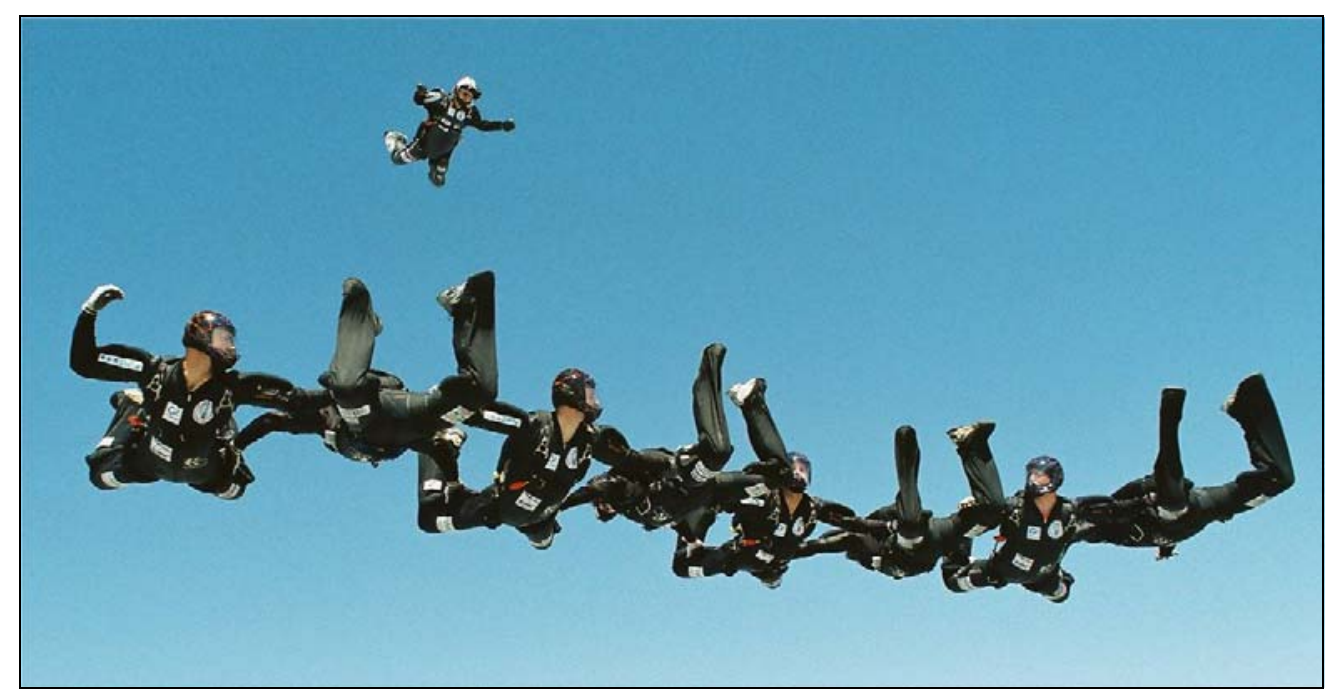

Abbildung 19: 8er-Mannschaft und Kameraflieger beim Formationsspringen (Foto: Edson Pacheco).

\footnotetext{
${ }^{79}$ Auch Block, Blockübergang oder Inter genannt.

${ }^{80}$ Z.B. um eine 360-Grad-Drehung aller einzelnen Springer, bevor die Endformation gebildet wird.

${ }^{81}$ Auch als Random oder Randomformation bezeichnet.
} 
Seit 2002 beträgt der Weltrekord in der 4er-Disziplin 42 (!) Formationen innerhalb der Arbeitszeit von 35 Sekunden; der Weltrekord in der 8er-Formation liegt seit 1997 bei 31 Punkten innerhalb der 50 Sekunden (vgl. BERGAN 2000, 2ff; FAI im Internet).

Die große Zahl der Formationen, die heutzutage von guten Mannschaften innerhalb der Arbeitszeit im Freifallformationsspringen geflogen werden, lässt erahnen, welche hohen kognitiven und motorischen Anforderungen an den einzelnen Sportler gestellt werden. In den folgenden Abschnitten folgt der - meines Wissens erstmalige - Versuch einer Einordnung dieser Anforderungen.

\subsubsection{Anforderungsprofil des Freifallformationsspringens}

Das Vorgehen bei dem Versuch einer Ermittlung des Anforderungsprofils des Freifallformationsspringens erfolgt anhand der von KIRCHNER und STÖBER (1994) vorgeschlagenen Systematik: Zunächst wird die betrachtete Sportart hinsichtlich von Ziel- und Bedingungsklassen eingeordnet - erst danach erfolgt die Definition des Anforderungsprofils (vgl. KIRCHNER/STÖBER 1994, 339).

Im Rahmen der Zielklassen lässt sich das Freifallformationsspringen sowohl der Klasse „Räumliche Ziele genau treffen“ als auch der Klasse „Bewegungen mit Normen übereinstimmend darbieten“ zuordnen (vgl. KIRCHNER/STÖBER 1994, 343): Für die beteiligten Freifallspringer kommt es in jedem Sprung wiederholt zunächst darauf an, eine bestimmte Position relativ zu den anderen Springern innerhalb der Formation anzufliegen und gezielt die geforderten Griffe zu nehmen. Die gebildeten Formationen und Blockübergänge müssen zudem fehlerfrei präsentiert werden.

Für eine Beschreibung der Bedingungsklassen sind innerhalb der Klasse der objektiven Gesetzmäßigkeiten zunächst die medienspezifischen Besonderheiten des Handelns in der Luft - im Speziellen die Luftwiderstandskräfte - von wesentlicher Bedeutung (vgl. KIRCHNER/ STÖBER 1994, 345): Diese Luftwiderstandskräfte, hervorgerufen durch den Fahrtwind während Absprung bzw. Freifallphase, stellen die einzige Möglichkeit für den Springer dar, seine Freifallbewegung zu steuern. Nur im Bereich des Leewirbels direkt über einem anderen Springer ist diese Steuerungsmöglichkeit nicht vorhanden. Weiterhin ist die große Höhe von 3000-4000m über Grund, in der das Freifallformationsspringen ausgeübt wird, anzuführen. Hieraus resultieren niedrigere Temperaturen und ein deutlich reduzierter Sauerstoffpartialdruck (vgl. Hollmann/Hettinger 2000, 458; Rost 2001a, 598). 
Im Rahmen der Bedingungsklasse der Kooperationsanforderungen kommt es beim Freifallformationsspringen hauptsächlich auf die zeitliche Abstimmung der Einzelhandlungen der beteiligten Fallschirmspringer an: Die geforderten Formationen müssen synchron gebildet und wieder geöffnet werden. Zudem gilt es während der Blockübergänge, die Bewegungsdynamik innerhalb der Untergruppen aufeinander abzustimmen.

Nach dieser Einordnung in Ziel- und Bedingungsklassen folgt in den nächsten Abschnitten eine Betrachtung des individuellen Anforderungsprofils.

\subsubsection{Konditionelle Anforderungen des Freifallformationsspringens}

Die motorischen Hauptbeanspruchungsformen Kraft, Ausdauer, Beweglichkeit und Schnelligkeit (vgl. MeINEL/SCHNABEL 1987, 242; BöS/MeCHLING 1983, 122f) stellen als leistungslimitierende Faktoren die Basis für die Leistung beim Freifallformationsspringen dar. Für die ersten drei konditionellen Fähigkeiten von Kraft, Ausdauer und Beweglichkeit sind Werte im Bereich eines Grundlagentrainings für eine gute Leistung im Freifallformationsspringen hinreichend. Hauptsächliche Kraftbeanspruchungen sind durch die Kraftarbeit gegen den Fahrtwind während der Exitaufstellung im Flugzeug, durch den Absprung selbst und im freien Fall durch die Arbeit gegen den Luftwiderstand gegeben. Ein Grundlagenniveau der Kraft sollte also ausreichend sein.

Ähnliches gilt für die Fähigkeit der Ausdauer: Eine gute Grundlagenausdauer hilft, die Belastungen eines Sprungtages (ständige Höhenakklimatisationen, Packen, Stress, Sprungvorbereitungen) besser zu ertragen - auch wenn man aufgrund der kurzen Zeitdauer der Leistungsbeanspruchung beim eigentlichen Freifallformationssprung (35 bzw. 50 Sekunden) zunächst keine Leistungsbestimmung durch die Ausdauer vermutet. An dieser Stelle sei aber auf den typischen Verlauf der Herzfrequenz mit (dem Springer in der Regel nicht bewussten) recht hohen Werten während eines Fallschirmsprungs verwiesen (siehe hierzu Abschnitt 5.1.1 auf Seite 108): Der Durchschnittswert der Herzfrequenz bei den im Rahmen der Untersuchungen durchgeführten Freifallformationssprüngen beträgt etwa 160 Schläge pro Minute, wobei es sich hierbei um die kombinierten Komponenten von Stressreaktion und körperlicher Belastung im Freifall handelt (vgl. FALK/BAR-ELI 1995, 116).

Der konditionellen Fähigkeit der Beweglichkeit kommt beim Freifallformationsspringen vornehmlich eine Rolle der Verletzungsprophylaxe zu. Großräumige Bewegungen finden 
nicht statt - somit muss die Beweglichkeit ebenfalls nicht über ein Grundlagenniveau hinaus trainiert werden.

Die konditionelle Fähigkeit der Schnelligkeit wiederum ist vor allem im Hochleistungsbereich sehr wohl ein leistungsbestimmender Faktor beim Freifallformationsspringen. So kann ab einem bestimmten Leistungsniveau ${ }^{82}$ eine Leistungssteigerung im Prinzip nur über eine Steigerung der Bewegungsschnelligkeit erreicht werden. Somit erhält hier die azyklische Schnelligkeit der Muskelbewegungen eine besondere Bedeutung.

$\mathrm{Zu}$ der Fähigkeit der Schnelligkeit werden häufig auch die Reaktionsschnelligkeit und die Antizipation hinzugezählt. Diese „Teilfähigkeiten“ der Schnelligkeit sind natürlich auf jedem Leistungsniveau ein leistungslimitierender Faktor und sollten bei Freifallformationsspringern hochgradig ausgeprägt sein. Eine Leistungssteigerung in jeder Erfahrungs- und Leistungsstufe erfolgt nämlich in besonderem Maße durch Fehlervermeidung bzw. durch schnelle Reaktion auf situative Gegebenheiten während eines Sprunges (oder die Antizipation derselben).

\subsubsection{Koordinative Anforderungen des Freifallformationsspringens}

Die in der Literatur aufgeführte Anzahl der koordinativen Fähigkeiten ist uneinheitlich:

MEINEL und SCHNABEL differenzieren die koordinativen Fähigkeiten in Orientierungsfähigkeit, Gleichgewichtsfähigkeit, Differenzierungsfähigkeit, Kopplungsfähigkeit, Rhythmisierungsfähigkeit, Reaktionsfähigkeit und Umstellungsfähigkeit (vgl. MEINEL/SCHNABEL 1987, 247). Diese koordinativen Fähigkeiten treten bei sportlichen Bewegungen stets im Komplex auf. Sie kommen nicht isoliert vor, sondern ergeben in bestimmten Verknüpfungen und Ausprägungen das Anforderungsprofil einer Sportart. Es folgt der Versuch einer Analyse für das Anforderungsprofil des Freifallformationsspringers.

Bei der Differenzierung der koordinativen Fähigkeiten, u.a. von Bös und MECHLING (1983), wurden in praktischen Untersuchungen im wesentlichen Turnübungen bzw. ähnliche Aufgabenstellungen im Bewegungsraum einer Turnhalle betrachtet (vgl. Bös/MECHLING 1983, 188ff). Es stellt sich die Frage, ob die Teildefinitionen und -anforderungen der so ermittelten koordinativen Fähigkeiten ohne Einschränkungen auf die „nicht bodengebundene“63 ${ }^{63}$ Sportart

\footnotetext{
${ }^{82}$ Etwa ab einem Durchschnitt von 12-13 Punkten innerhalb der Arbeitszeit, sowohl in der Vierer- als auch in der Achterdisziplin.

${ }^{83}$ Zwischen Sportarten mit und ohne Bodenkontakt ergeben sich z.B. Unterschiede hinsichtlich der Feedbackregulation (vgl. WEDER 2002, 27).
} 
des Freifallformationsspringens mit ihren ganz eigenen Besonderheiten und Charakteristika übertragbar sind. Für die folgende Einordnung des Freifallformationsspringens verstehen sich die angegebenen koordinativen Fähigkeiten daher vor allem als Strukturierungshilfe.

Im Rahmen von Orientierungs- und Gleichgewichtsfähigkeit ist das sportartspezifische Anforderungsprofil des Freifallformationsspringers zunächst mit dem der anderen Freifallsportarten vergleichbar: Der Freifallformationsspringer muss sich stabilisieren und sich im dreidimensionalen Raum orientieren. Dies sind Grundfertigkeiten, die er im Rahmen seiner sportlichen Ausbildung erlernt haben sollte. Dabei stellt die Orientierung im dreidimensionalen Raum vor dem Hintergrund der Bewegungsschnelligkeit mitunter eine besondere Schwierigkeit dar: In Situationen mit hoher Geschwindigkeit und/oder hoher Komplexität kann das kognitive und emotionale Regulationssystem überfordert werden (vgl. WEDER 2002, 174). Zusätzlich fehlt mitunter eine direkte taktile Rückmeldung über ausgeführte Körperbewegungen und deren Wirkungen, da die Bewegung im freien Fall erfolgt (vgl. WEDER 2002, 27): Einzig die Richtung und Stärke der relativen Luftanströmung vermag dem Freifallformationsspringer Rückmeldungen über die momentane Körperposition zu geben.

Betrachtet man dagegen die Kopplungs- und Differenzierungsfähigkeit, so sind sportartspezifische Merkmale als leistungsbestimmende Faktoren des Freifallformationsspringens zu erkennen. Diese koordinativen Fähigkeiten sollten in hohem Maße ausgeprägt sein und dienen einer optimalen Bewegungsökonomisierung im freien Fall (also einer optimalen Feinabstimmung von Kraftimpulsen bei der Einzel- oder Gruppenbewegung). Aufgrund der Vielzahl an erforderlichen Bewegungen, die sich jeweils auf feinster Ebene voneinander unterscheiden, wird so die Kopplungs- und Differenzierungsfähigkeit für das Freifallformationsspringen zu einem leistungslimitierenden Faktor.

Die „reaktiven“ koordinativen Fähigkeiten (Reaktionsfähigkeit und Umstellungsfähigkeit) bilden eine zweite Gruppe der leistungslimitierenden Faktoren: Der Freifallformationsspringer muss $\mathrm{zu}$ jeder Zeit auf in den drei Dimensionen auftretende Abstände und Unregelmäßigkeiten reagieren - weiterhin muss er in der Lage sein, möglichst schnell auf besondere, unvorhergesehene Situationen und Abweichungen ${ }^{84} \mathrm{zu}$ reagieren (Umstellungsfähigkeit).

\footnotetext{
${ }^{84}$ Wie z.B. Fehler von Mannschaftsmitgliedern (vgl. JEFFERIES 1998b).
} 
Zusätzlich ist im Rahmen der Antizipation mitunter eine hohe Gedächtnisleistung gefordert. So beginnt man nach der letzten Formation einer Sequenz zwar wieder bei dem ersten Punkt, müsste sich also nur maximal sechs Aufgaben- oder Bewegungsprogramme zu den jeweiligen Formationen pro Sprung merken. Leider jedoch vertauschen etliche der Blockübergänge die Positionen im Sprung. So kann es - je nach Auslosung - geschehen, dass ein Sprung ,nicht aufgeht“", da ein Zurücktauschen während eines freien Übergangs aus Zeitgründen nicht in Frage kommt. Die Zahl der zu merkenden Aufgaben oder Formationen kann so bis auf 24 anwachsen, zehn oder zwölf zu merkende Punkte pro Sprung sind keine Seltenheit. Häufig beobachtet man bei Formationsspringern daher auch den gefürchteten sogenannten „Brainlock“ - das plötzliche Vergessen der nächsten Bewegungsaufgabe.

Hierdurch können sekundenlange „Aussetzer“ entstehen, die den Rhythmus eines ganzen Sprungs zerstören können (vgl. HAMILTON 1997).

Eine hieraus resultierende wesentliche leistungsbestimmende Rolle der Konzentrationsfähigkeit und Aufmerksamkeit wird u.a. von WEDER (2002) am Beispiel von Sportarten, die durch hohe Geschwindigkeiten charakterisiert werden ${ }^{85}$, bestätigt (vgl. WEDER 2002, 177).

Da das Formationsprogramm aus 38 verschiedenen Elementen ${ }^{86}$ besteht, ergibt sich während der Auslosung eines Wettkampfes eine riesige Anzahl von Kombinationsmöglichkeiten für die fünf oder sechs Formationen enthaltenden Sequenzen der Sprünge. Dadurch wird es für jede Mannschaft im Wettbewerb immer wieder $\mathrm{zu}$ „unbekannten“ freien Übergängen kommen, denn die große Anzahl der Kombinationsmöglichkeiten ist - im Gegensatz zu den Blockübergängen - mit herkömmlichen Mitteln nicht trainierbar. Somit müssen in einem Wettkampf viele der freien Formationsübergänge quasi auf Anhieb, ohne vorheriges Training, gelingen. Dabei steht der einzelne Springer häufig unter einem hohen Erwartungsdruck: Freie Übergänge sind die Basis für ein hohes Punkteergebnis im Sprung, da sie in der Regel schneller zu fliegen sind als Blockübergänge.

Im Rahmen der Blockübergänge werden ferner hauptsächlich Drehungen oder Translationen zusammenhängender Untergruppen ${ }^{87}$ gefordert. Somit muss eine Feinkoordination der individuellen Bewegung innerhalb der jeweiligen Untergruppe erfolgen, es ist eine Synchronisation der Einzelbewegungen erforderlich. Also kommt auch der koordinativen Fähigkeit der

\footnotetext{
${ }^{85}$ Auto- und Motorradrennen, Bob- und Skisport, Militär- und Berufspiloten, davon ein Fallschirmspringer (vgl. WEDER 2002, 22).

${ }^{86} 16$ freie Formationen und 22 Blöcke mit jeweils einer Ausgangs- und einer Endformation.

${ }^{87}$ Zweier- oder Dreiergruppen - in der 8er-Disziplin auch bis zu Vierergruppen.
} 
Rhythmisierungsfähigkeit im Rahmen dieser Synchronisation beim Freifallformationsspringen eine besondere Bedeutung zu.

Weiterhin muss der Fallschirmspringer, der sich gerade in einer Formation befindet, kontrollieren, ob alle Griffe korrekt genommen sind. Danach antizipiert er seine Bewegung zur nächsten geforderten Formation und gibt ein Zeichen zum Lösen der aktuellen Formation (engl. „key“). Alle Springer müssen sich nun zu einer neuen Position in der Luft relativ zu einem gedachten Zentrumspunkt bewegen, die Bewegung abstoppen und die geforderten Griffe der neuen Formation aufnehmen. Während jeder Bewegung und in jeder Formation müssen dabei fortwährend Unterschiede in der Fallgeschwindigkeit und sonstige Unruhen ausgeglichen werden. Gleichzeitig gilt es aber, alle diese Aufgaben so schnell wie möglich zu absolvieren (vgl. JEFFERIES 1998b; WEDER 2002, 170).

Abschließend stellt das Beherrschen der Ausübung einer Sportart wie des Freifallformationsspringens in der dritten Dimension sowohl einen besonderen Reiz als auch eine große Schwierigkeit dar. Die dritte Dimension, die in vertikalen Übergängen beim Freeflying und Formationsspringen bewusst kontrolliert wird, ist sonst nur beim Sporttauchen oder Unterwasserrugby erfahrbar. So ist es bei einigen Blockübergängen im Formationsspringen aus Gründen der Zeitersparnis äußerst sinnvoll, mit bewussten Höhenunterschieden zu fliegen. So kann der kürzeste Weg über einen anderen Springer hinweg ${ }^{88}$ realisiert werden. Abbildung 20 auf der folgenden Seite zeigt einen solchen vertikalen Übergang. Man erkennt das geforderte Zwischenbild (Inter) aus Abbildung 18 (Seite 80): Die beiden in dieser Grafik dunkel eingefärbten Formationsspringer fliegen aus dem Zentrum direkt über ihre Mannschaftskameraden hinweg in ihre neuen Außenpositionen.

Solche Abkürzungen und Zeitersparnisse durch Ausnutzung des Dreidimensionalen lassen sich auch im Bereich der in festen Übergängen geforderten Drehungen von Untergruppen erzielen: So ist die Drehung einer Untergruppe stets als Drehung relativ zu der anderen Untergruppe definiert. Verschiebt man die Untergruppen während der Drehung im freien Fall übereinander ${ }^{89}$, so lässt sich die Drehung erheblich verkürzen. Dies macht sich in einer deutlichen Zeitersparnis und einer größeren Anzahl von Formationen innerhalb der Arbeitszeit bemerkbar.

\footnotetext{
${ }^{88}$ Anstatt die konventionelle (längere) Flugbahn um den anderen Springer herum zu wählen.

${ }^{89}$ Üblicherweise mit einem Höhenunterschied von 0,5-1,5 Metern.
} 


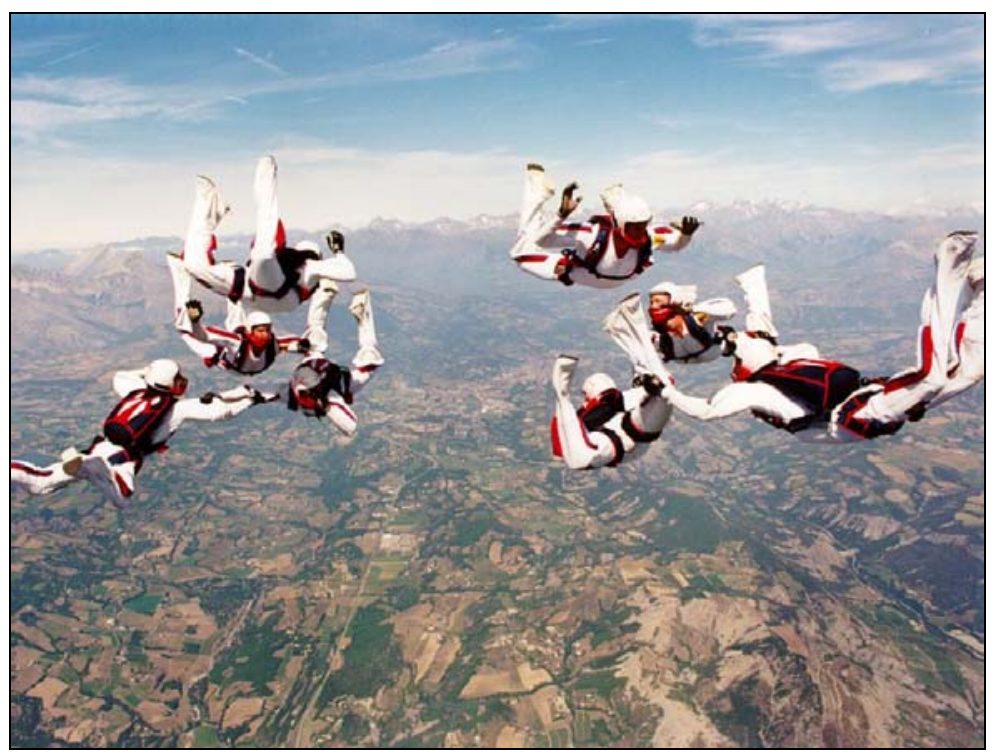

Abbildung 20: Blockübergang Nr. 22 der amerikanischen 8er-Nationalmannschaft (Foto: Tim Wagner).

Da der Formationsspringer im freien Fall jedoch zur Steuerung seines Körpers auf die durch die Fallgeschwindigkeit resultierende Luftanströmung angewiesen ist, stellt eine Vertikalbewegung eine besondere Schwierigkeit dar: In dem Moment, in dem sich der Springer direkt über dem unteren Sportler befindet, passiert er dessen Leewirbel - eine Zone mit deutlich verringerter Luftanströmung. Es gilt diese Zone schnell zu ,überspringen“, denn innerhalb dieses Leewirbels ist ein Springer nicht handlungsfähig. Er droht, auf den darunter Freifallenden $\mathrm{zu}$ stürzen und so die gesamte Formation zum Zusammenstürzen (engl. „funnel“") zu bringen.

Zusammenfassend lässt sich damit folgern, dass aufgrund der Komplexität von kognitiven Bewertungs- und Entscheidungsprozessen und dem hohen Anteil feinkoordinierter Bewegungen beim Freifallformationsspringen damit zu rechnen ist, dass in dieser Sportart die koordinativen Fähigkeiten hochgradig leistungsbestimmend sind (vgl. CLANCY 1999, 19).

In Anlehnung an die Klassifizierung der Sportarten hinsichtlich ihrer spezifischen Anforderungen nach OXENDINE (1970) und TAYLOR (1987) wäre somit ein optimales Erregungsniveau für das Freifallformationsspringen mit niedrigen oder mittleren Angstwerten verknüpft. Die weiteren Freifalldisziplinen Freestyle, Skysurfen und Freeflying erscheinen hinsichtlich ihrer typischen Anforderungen und der ebenfalls vorhandenen Mannschaftswertung und dreidimensionalen Bewegungsgestaltung ähnlich, sodass für diese Disziplinen ebenfalls ein niedriges Erregungsniveau für maximale Leistungen optimal erscheint.

Auf die Limitierungen einer solchen Einordnung (siehe Abschnitt 3.2.2.1 auf Seite 41) sei an dieser Stelle aber nochmals hingewiesen. 


\section{Empirischer Teil}

\section{Fragestellungen}

In der sportwissenschaftlichen und medizinischen Literatur lassen sich mehrere Untersuchungen finden, die sich mit der Angst beim Fallschirmspringen beschäftigen - einige behandeln auch den Angst-Leistungs-Zusammenhang bei Fallschirmspringern. Die Untersuchungen verwendeten dabei allerdings recht unterschiedliche Fragestellungen. Die Vielfalt der Herangehensweisen reicht von der Betrachtung physiologischer Aspekte (SHANE/SLINDE 1968, ReID et al. 1971, DeroAnne et al. 1975, Kopp et al. 1978, AnFILOGOFF et al. 1987, SCHEDlOwski/Tewes 1992, Richter et al. 1996, Roth et al. 1996, DeINZER et al. 1997) und Sportverletzungen beim Fallschirmspringen (BAR-DAYAN/SHEMER 1998, KRAGH/TAYLOR 1996, LEPSIEN et al. 1998) über die Untersuchung des Appetenz-Aversions-Konflikts (FENZ/EPSTEIN 1967, KARGE 1994) und Angstunterschieden hinsichtlich verschiedener Angstbewältigungsstrategien, Erfahrungsstufen und Ängstlichkeitstypen (URSIN et al. 1978, GAL-Or et al. 1985, SHARMA et al. 1994, BÖCKHELER 1995, FALK/BAR-ELI 1995, BREIVIK et al. 1998) bis hin zum Angst-Leistungs-Zusammenhang (FENZ/JONES 1972, POWELL/VERNER 1982, IDZIKOWSKI/ BADDELEY 1987, ENDER et al. 1992, SHARMA et al. 1994) bei Fallschirmspringern (vgl. MAUSHAKE 1987, 55; MUTHNY/ SÜSS 2003, 241).

Es hat jedoch den Anschein, dass sich alle diese Studien nur deshalb mit dem Fallschirmspringen beschäftigen, um eine reproduzierbare Situation mit einer hohen erwarteten Zustandsangst für die Untersuchung nutzen zu können ${ }^{90}$. Diese These lässt sich durch die Tatsache stützen, dass oft nur Sprunganfänger oder sehr unerfahrene Fallschirmspringer betrachtet werden - anscheinend wiederum mit dem Ziel, erfahrene Springer „auszublenden“ und eine Testsituation mit hohen Angstwerten der Anfänger zu provozieren. Zwar betrachten einige der Untersuchungen auch erfahrene Springer, jedoch genügt hier bereits eine Sprungzahl von 100 bzw. 300 Sprüngen, um als „sehr erfahren“ zu gelten (vgl. FENZ/JONES 1972, 1; BÖCKHELER 1995, 83). Eine ernsthafte sportwissenschaftliche Beschäftigung mit dem Fallschirmspringen wird jedoch schnell vermisst - einzig in den Arbeiten von MÜLLER (1989) findet sich eine erste genauere theoretische Behandlung des Fallschirmspringens ,ohne Hintergedanken“.

\footnotetext{
${ }^{90}$ Eine Ausnahme gilt hier für die Kategorie der sportmedizinischen Untersuchungen.
} 
In den im Folgenden beschriebenen Untersuchungen wird aus Gründen einer besseren Vergleichbarkeit ausschließlich das Freifallformationsspringen betrachtet. Zentrale Fragestellung ist hierbei die Überprüfung der Anwendbarkeit der Katastrophentheorie zur Beschreibung des Angst-Leistungs-Zusammenhangs beim Fallschirmspringen. Es soll versucht werden, ein für eine maximale Leistung optimales Erregungs- bzw. Angstniveau, wie es auch innerhalb der Katastrophentheorie vorhergesagt wird, zu identifizieren und quantitativ zu beschreiben.

Um ein solches optimales Erregungsniveau zu definieren, wäre ein differentialpsychologischer ${ }^{91}$ Ansatz wünschenswert, um möglichst genaue Ergebnisse zu erzielen - schließlich wird der Angst-Leistungs-Zusammenhang durch weitere Einflussgrößen moderiert (vgl. Abschnitt 3.2 auf Seite 35). Ein solches Vorgehen wird u.a. von COHEN et al. (2003) empfohlen (vgl. COHEN et al. 2003, 146).

$\mathrm{Zu}$ einer vereinfachten Betrachtung dieser zentralen Fragestellung werden im Folgenden trotzdem nicht einzelne Fallschirmspringer, sondern Gruppen von Probanden gleicher Eigenschaftsangst (Ängstlichkeit) betrachtet, um den Umfang dieser Untersuchungen zu begrenzen $^{92}$. Es soll untersucht werden, ob sich innerhalb dieser Ängstlichkeitsgruppen Unterschiede des Angst-Leistungs-Zusammenhangs nachweisen lassen, die ein gruppenspezifisches optimales Erregungsniveau erwarten ließen.

Nach LauX/GlanZManN (1996) scheint die Intensität einer Angstreaktion in Situationen physischer Gefährdung unabhängig von der Ängstlichkeit des Probanden zu sein ${ }^{93}$.

Eine weitere Frage ist daher, ob auch fertig ausgebildete und erfahrene Fallschirmspringer sowie solche mit einer hohen bzw. niedrigen Eigenschaftsangst stets den gleichen zeitlichen Angstverlauf bei einem Sprung zeigen, wie man es bei Anfängern oder Unerfahrenen vermutet. Wäre dies der Fall, könnte man die Suche nach einem optimalen Erregungsniveau als beendet ansehen, da in der untersuchten Situation die gemessene Angst nur aus der Komponente der physischen Gefährdung und eben nicht aus Anteilen der Wettkampfangst bestünde. Nur besondere Angstbewältigungsstrategien oder spezielles „beruhigendes“ Training ${ }^{94}$ würden es dann ermöglichen, niedrigere Angstwerte und damit potentiell bessere Leistungen im Sprung zu erzielen.

\footnotetext{
${ }^{91}$ Die differentielle Psychologie betrachtet psychologische Fragen unter dem Aspekt individueller Unterschiede.

92 Siehe hierzu auch NOTEBOOM et al. 2001, 2099.

${ }^{93}$ Siehe hierzu Abschnitt 1.1.2 auf Seite 6.

${ }^{94}$ Dies könnte z.B. mentales bzw. autogenes Training sein (vgl. DeRosAliA 2000, 99f; BIEDERMANN 2002, $90 f$ ).
} 
Neben dem Blick auf erfahrene und weniger erfahrene Springer wird in der logischen Konsequenz im Folgenden auch ein Unterschied hinsichtlich der Angst zwischen Trainingsund Wettkampfssituationen untersucht. Solch eine Differenz wäre ebenfalls in der „einheitlichen Situation“ hoher Zustandsangst beim Fallschirmspringen nicht nachweisbar; sie würde quasi durch die hohen Angstwerte bei physischer Gefährdung „überdeckt“ (vgl. LAUX/ GLANZMANN 1996, 117).

Abschließende Fragestellung ist die Untersuchung von positiven Effekten der Selbstwirksamkeitsüberzeugung und des Erfahrungsstandes der Probanden.

Sowohl HARDY (mit seinem Schmetterlingskatastrophenmodell 1990) als auch PAIN (1999) und EDWARDS et al. (2002) setzten die Selbstwirksamkeit so ein, dass sie das Auftreten von Katastropheneffekten verzögerte. Alle konnten positive bzw. verzögernde Effekte der Selbstwirksamkeit auf den Angst-Leistungs-Zusammenhang nachweisen, HARDY bezeichnete jedoch seinen ursprünglichen Ansatz selbst als „spekulativ“, und PAIN hatte mathematische bzw. statistische Schwierigkeiten in der Überprüfung des Katastrophenmodells. Sie versuchte, über die graphische Darstellung ihrer Messwerte bzw. über die Untersuchung bimodalen Verhaltens der Zustandsvariable ein Katastrophenmodell von Angst und sportlicher Leistung nachzuweisen (vgl. PAIN 1999, 1).

Das Einarbeiten von Selbstwirksamkeitsüberzeugung und Sprungerfahrung der Probanden soll im Folgenden sowohl durch Testen einer Schmetterlingskatastrophe ${ }^{95}$ als auch durch Testen von „einfachen“ Spitzenkatastrophen geschehen. Wenn die Selbstwirksamkeit tatsächlich die Eigenschaft hat, Katastropheneffekte zu verzögern, so wäre sie zum Einen als Verschiebungsfaktor $c$ innerhalb des Schmetterlingskatastrophemodells bestätigt. Zum Anderen sollten aber Verzögerungen der Katastropheneffekte auch als unterschiedliche Spitzenkatastrophenmodelle bei verschiedener Selbstwirksamkeitsüberzeugung nachweisbar sein.

Eine identische Vorgehensweise soll diese Effekte für die unterschiedliche Sprungerfahrung der Probanden überprüfen: Auch hier stellt sich nicht nur die Frage, ob hoch erfahrene Springer weniger oder ebenso ängstlich sind wie unerfahrene Springer gleicher Eigenschaftsangst, sondern ebenfalls, ob die Erfahrung auch als Faktor zu sehen ist, der das Auftreten von Katastropheneffekten verhindert.

\footnotetext{
${ }^{95}$ Wie von COHEN et al. (2003) empfohlen (vgl. COHEN et al. 2003, 157).
} 


\section{Hypothesenbildung}

Folgende Hypothesen lagen den Untersuchungen von Freifallformationsspringern zugrunde, wobei Training solche Übungssprünge bezeichnet, die in den Tagen unmittelbar vor einem Wettkampf am Austragungsort des Wettbewerbes durchgeführt wurden:

H1: Formationsspringer sind im Wettkampf aufgeregter bzw. ängstlicher als im Training.

H2: Zwischen der 4er- und der 8er-Disziplin existieren keine Unterschiede, was Erregungs- oder Leistungswerte der Formationsspringer betrifft.

H3: Es existieren Differenzen von Angst- oder Leistungswerten hinsichtlich des Geschlechts.

H4: Hocherfahrene Formationsspringer erzielen eine bessere Leistung und haben niedrigere Angstwerte als wenig erfahrene Freifallformationsspringer.

H5: Freifallformationsspringer mit einer hohen Eigenschaftsangst (Ängstlichkeit) haben beim Fallschirmspringen stets höhere Werte der Zustandsangst als Springer mit einer niedrigen Eigenschaftsangst.

H6: Die Angstkomponente der Selbstwirksamkeitsüberzeugung hat einen positiven Einfluss auf den Angst-Leistungs-Zusammenhang.

H7: Maximale Leistungen beim Formationsspringen sind (aufgrund der hohen Aufgabenkomplexität) mit niedrigen Angstwerten verknüpft.

H8: Der Zusammenhang zwischen Angst und sportlicher Leistung bei Freifallformationsspringern lässt sich am deutlichsten durch ein Katastrophenmodell beschreiben.

Die Fragestellungen, ob bei Fallschirmspringern mit einer hohen Eigenschaftsangst früher Katastropheneffekte auftreten als bei solchen mit einer niedrigen Eigenschaftsangst und ob hohe Werte der Selbstwirksamkeitsüberzeugung oder der Sprungerfahrung vor dem Eintreten von Katastropheneffekten schützen können, sind nur qualitativ bzw. nicht mit den bekannten statistischen Tests zu beantworten. Daher erfolgt für diese Fragen keine Hypothesenbildung im Rahmen der Anpassung von Katastrophenflächen an die Messwerte wird aber auf diese beiden Fragen in besonderem Umfang eingegangen. 


\section{Untersuchungsmethodik}

Die Untersuchungen zum optimalen Erregungsniveau für das Freifallformationsspringen wurden im Rahmen von drei Wettbewerben durchgeführt. Dies waren die Weltmeisterschaften Formationsspringen im Oktober 1999 in Corowa, Australien sowie die Deutschen Meisterschaften im August 2000 in Gera, Thüringen und im August 2002 in Gransee, Brandenburg.

\subsection{Beschreibung der Probandengruppe}

Formationsspringer, die an den oben genannten Wettkämpfen teilnahmen, wurden gefragt, ob sie an einer sportwissenschaftlichen Untersuchung teilnehmen würden. Hierbei wurde ihnen die Voraussetzung, bei Trainings- und Wettbewerbssprüngen ein Gerät zur Messung und Speicherung der Herzfrequenz zu tragen und nach der Landung jeweils einen Fragebogen auszufüllen, mitgeteilt. Alle Befragten bildeten eine Gruppe von insgesamt 88 Probanden aus 10 Nationen (30 Frauen und 58 Männer), die 218 Sprünge im Rahmen der Untersuchungen durchführten. Das Alter der Probanden betrug durchschnittlich 34,0(63) Jahre ${ }^{96}$ (Min.: 19; Max.: 58), die Sprungerfahrung gemittelt 2537(2304) Sprünge (Min.: 290; Max.: 13000).

Vor Beginn der Untersuchung füllten die Probanden einen Fragebogen zu allgemeinen Grunddaten wie Alter, Geschlecht, Sprungerfahrung und weiteren Größen sowie einen Bogen mit der Trait-Anxiety Skala des STAI nach SPIELBERGER zur Ermittlung der Eigenschaftsangst aus. Beide Fragebögen finden sich im Anhang dieser Arbeit. Im Rahmen der weiteren Betreuung der Testpersonen wurde der persönliche Ruhepuls, jeweils sofort nach dem Erwachen am Morgen, festgestellt.

Die Probanden wurden dann anhand ihrer Eigenschaftsangst in drei Gruppen, jeweils nach niedriger, mittlerer und hoher Ängstlichkeit ${ }^{97}$, aufgeteilt.

Da die Probanden im Rahmen der Untersuchungen unterschiedlich viele Sprünge durchführten, ist die Anzahl der Probanden pro Gruppe sehr unterschiedlich. Die Einteilung der Gruppen erfolgte jedoch so, dass die Gesamtzahl der beobachteten Sprünge pro Gruppe einheitlich bei etwa 70 beobachteten Sprüngen pro Ängstlichkeitsgruppe liegt.

\footnotetext{
${ }^{96} \mathrm{Vgl}$. Anm. 10 auf Seite 11.

${ }^{97} \mathrm{Zu}$ beachten ist, dass es sich hierbei um relative Werte handelt.
} 
Tabelle 6 bis Tabelle 8 zeigen die Gruppeneinteilungen der Probanden ${ }^{98}$, sowie Geschlecht, Disziplin $^{99}$, Sprungerfahrung und die Anzahl der durchgeführten Sprünge.

Tabelle 6: Die Probanden der Gruppe 1: Niedrige Ängstlichkeit.

\begin{tabular}{|c|c|c|c|c|c|c|}
\hline $\begin{array}{l}\text { Ängstlichkeits- } \\
\text { gruppe }\end{array}$ & Proband & $\mathbf{m} / \mathbf{w}$ & Alter & Disziplin & $\begin{array}{l}\text { Sprung- } \\
\text { erfahrung }\end{array}$ & $\begin{array}{c}\text { Sprünge im } \\
\text { Rahmen der } \\
\text { Untersuchungen }\end{array}$ \\
\hline \multirow{32}{*}{ 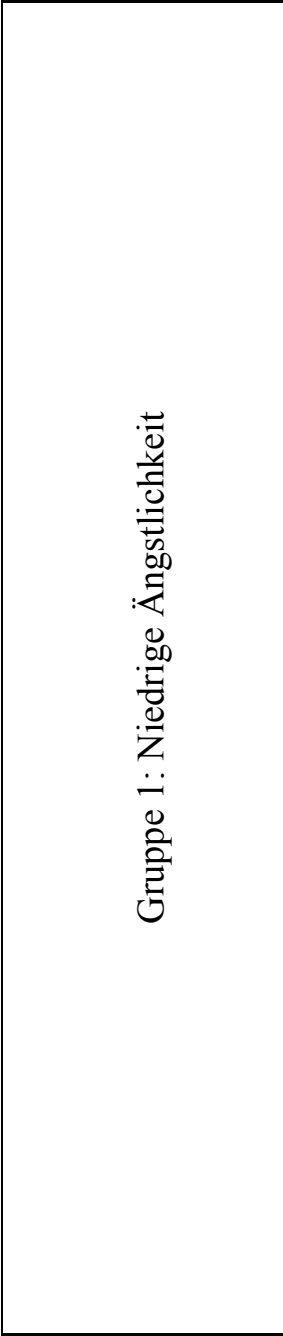 } & ClD-Just4-D & $\overline{\mathrm{w}}$ & 31 & 4 & 295 & 2 \\
\hline & StD-Just4-D & $\mathrm{m}$ & 33 & 4 & 350 & 2 \\
\hline & SaK-JWM8-D & $\mathrm{w}$ & 24 & 8 & 600 & 2 \\
\hline & TiE-Last4-D & $\mathrm{w}$ & 26 & 4 & 730 & 2 \\
\hline & DiB-Just4-D & $\mathrm{m}$ & 38 & 4 & 740 & 3 \\
\hline & AnL-Fear4-D & $\mathrm{w}$ & 28 & 4 & 950 & 1 \\
\hline & FrH-Bund4-D & $\mathrm{m}$ & 33 & 4 & 960 & 2 \\
\hline & StS-Teamo8-D & $\mathrm{m}$ & 32 & 8 & 980 & 2 \\
\hline & NiE-4Linkfa-D & $\mathrm{w}$ & 33 & 8 & 1000 & 2 \\
\hline & KaS-Heho8-D & $\mathrm{w}$ & 24 & 8 & 1200 & 1 \\
\hline & MaA-SUI4-D & $\mathrm{m}$ & 29 & 4 & 1280 & 5 \\
\hline & ChS-Phos4-D & $\mathrm{m}$ & 31 & 4 & 1500 & 2 \\
\hline & UwS-Team4-D & $\mathrm{m}$ & 41 & 4 & 1500 & 2 \\
\hline & UwR-Phos4-D & $\mathrm{m}$ & 41 & 4 & 1500 & 2 \\
\hline & AcB-Phos4-D & $\mathrm{m}$ & 51 & $4 / 8$ & 1700 & 2 \\
\hline & RoK-Der4-D & $\mathrm{m}$ & 28 & 4 & 2300 & 2 \\
\hline & SiH-LO8-D & $\mathrm{W}$ & 36 & 8 & 2500 & 2 \\
\hline & ThK-Xray4-D & $\mathrm{m}$ & 32 & 4 & 2800 & 1 \\
\hline & HeS-GER8-D & $\mathrm{m}$ & 33 & 8 & 2900 & 5 \\
\hline & BeH-NDL4/8-EN & $\mathrm{m}$ & 35 & $4 / 8$ & 2900 & 7 \\
\hline & VoW-Sala4-D & $\mathrm{m}$ & 33 & 4 & 3000 & 1 \\
\hline & RaM-ESP4/8-SP & $\mathrm{m}$ & 34 & $4 / 8$ & 3500 & 2 \\
\hline & MaS-Eigh8-D & $\mathrm{m}$ & 45 & 8 & 3700 & 4 \\
\hline & HoS-GER8-D & $\mathrm{m}$ & 33 & 8 & 3800 & 2 \\
\hline & UlG-Fris4-D & $\mathrm{m}$ & 58 & 4 & 4000 & 2 \\
\hline & RaS-Tis4/8-D & $\mathrm{m}$ & 36 & $4 / 8$ & 4050 & 3 \\
\hline & LiA-NOR4-NO & $\mathrm{w}$ & 33 & 4 & 5100 & 3 \\
\hline & PiW-GER4-D & $\mathrm{m}$ & 42 & 4 & 6150 & 2 \\
\hline & IvC-ESP4/8-EN & $\mathrm{m}$ & 35 & $4 / 8$ & 8200 & 5 \\
\hline & ThS-GER4-D & $\mathrm{m}$ & 38 & 4 & 9400 & 2 \\
\hline & KaW-GER8-D & $\mathrm{m}$ & 40 & 8 & 10200 & 1 \\
\hline & JaJ-USA4/8-EN & $\mathrm{m}$ & 34 & $4 / 8$ & 13000 & 2 \\
\hline
\end{tabular}

Die Gruppe 1 der Probanden mit einer niedrigen Eigenschaftsangst ${ }^{100}$ besteht aus 8 Frauen und 24 Männern. Das Durchschnittsalter beträgt 35,0(72) Jahre, die mittlere Sprungerfahrung 3212(3103) Sprünge. Abbildung 21 zeigt die Verteilung von Alter und Sprunganzahl innerhalb dieser Gruppe.

${ }^{98}$ Die mittleren Stellen des Probandenkürzels geben an, aus welcher Mannschaft der Proband stammt. Die letzte Stelle des Kürzels bezeichnet die Sprache der verwendeten CSAI-2- und STAI-Fragebögen: D steht für Deutsch, EN für Englisch, SP für Spanisch, NO für Norwegisch und RU für Russisch.

${ }^{99}$ Einige der Probanden starteten gleichzeitig mit verschiedenen Mannschaften sowohl in der 4er- als auch der 8er-Wertung. Daher sind mehrere Einträge in den Disziplinspalten von Tabelle 6 bis Tabelle 8 möglich.

100 Ängstlichkeitswerte $\leq 29$ auf der Trait-Skala des STAI. 


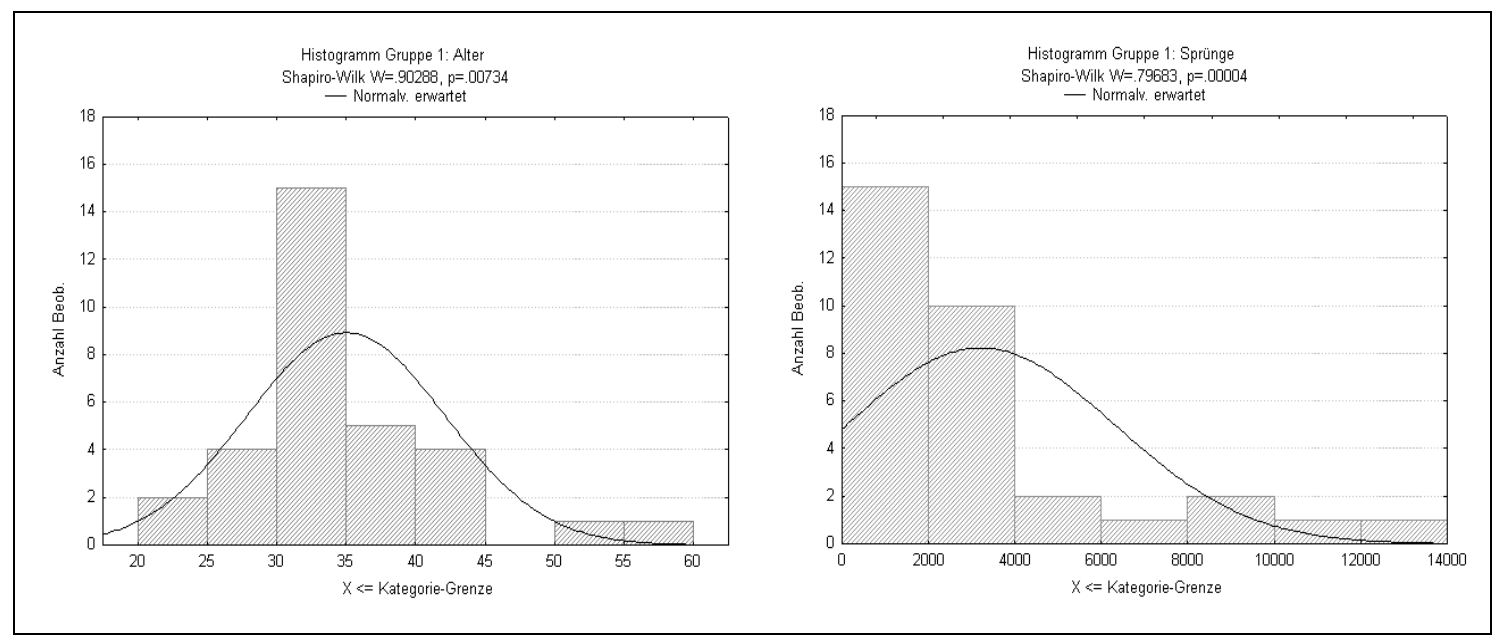

Abbildung 21: Histogramme von Alter und Sprungerfahrung der Gruppe 1 mit niedriger Eigenschaftsangst.

Tabelle 7: Die Probanden der Gruppe 2: Mittlere Ängstlichkeit.

\begin{tabular}{|c|c|c|c|c|c|c|}
\hline $\begin{array}{l}\text { Ängstlichkeits- } \\
\text { gruppe }\end{array}$ & Proband & $\mathbf{m} / \mathbf{w}$ & Alter & Disziplin & $\begin{array}{l}\text { Sprung- } \\
\text { erfahrung }\end{array}$ & $\begin{array}{c}\text { Sprünge im } \\
\text { Rahmen der } \\
\text { Untersuchungen }\end{array}$ \\
\hline \multirow{25}{*}{ 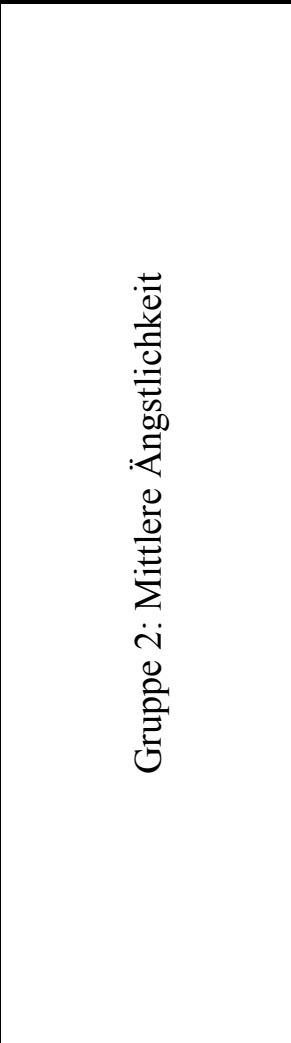 } & GeH-CYP4-EN & $\overline{\mathrm{m}}$ & 28 & $\overline{4}$ & 450 & 5 \\
\hline & SuW-Heho8-D & $\mathrm{w}$ & 31 & 8 & 730 & 2 \\
\hline & JuW-Eigh8-D & $\mathrm{m}$ & 35 & 8 & 800 & 3 \\
\hline & AnK-Dfv4-D & $\mathrm{m}$ & 26 & 4 & 890 & 2 \\
\hline & InL-Teamo8-D & $\mathrm{w}$ & 31 & 8 & 900 & 2 \\
\hline & AnK-Grou4-D & $\mathrm{m}$ & 34 & 4 & 950 & 3 \\
\hline & BiB-Grou4-D & $\mathrm{w}$ & 31 & 4 & 1000 & 3 \\
\hline & SoK-DEN4-EN & $\mathrm{m}$ & 33 & 4 & 1380 & 2 \\
\hline & HeA-Phos4-D & $\mathrm{w}$ & 38 & 4 & 1400 & 2 \\
\hline & VoS-Teamo8-D & $\mathrm{m}$ & 30 & 8 & 1400 & 2 \\
\hline & AnC-Schn4-D & $\mathrm{w}$ & 35 & 4 & 1500 & 2 \\
\hline & NiK-SUI4-D & $\mathrm{w}$ & 37 & 4 & 1600 & 3 \\
\hline & JeG-EADS4-D & $\mathrm{m}$ & 34 & 4 & 1700 & 2 \\
\hline & DoP-AUS8-EN & $\mathrm{m}$ & 31 & 8 & 2200 & 2 \\
\hline & AxR-Der4/8-D & $\mathrm{m}$ & 34 & 8 & 2500 & 2 \\
\hline & OlH-Hi5-D & $\mathrm{m}$ & 32 & 4 & 3000 & 1 \\
\hline & StS-RS4/8-D & $\mathrm{m}$ & 29 & $4 / 8$ & 3100 & 8 \\
\hline & NoW-Sala4-D & $\mathrm{m}$ & 39 & 4 & 3400 & 2 \\
\hline & UlS-RS4/8-D & $\mathrm{m}$ & 44 & $4 / 8$ & 3600 & 4 \\
\hline & ThV-NOR4-NO & $\mathrm{m}$ & 26 & 4 & 4000 & 1 \\
\hline & StG-BEL4/8-EN & $\mathrm{m}$ & 33 & $4 / 8$ & 4000 & 2 \\
\hline & OlB-Rs4/8-D & $\mathrm{m}$ & 30 & $4 / 8$ & 4100 & 6 \\
\hline & MaM-GER8-D & $\mathrm{m}$ & 39 & 8 & 4900 & 2 \\
\hline & VaP-RUS8-RU & $\mathrm{m}$ & 28 & 8 & 6000 & 1 \\
\hline & AlM-RUS8-RU & $\mathrm{m}$ & 33 & 8 & 6200 & 1 \\
\hline
\end{tabular}

Die zweite Gruppe ${ }^{101}$ besteht aus 6 Frauen und 19 Männern mit einem Durchschnittsalter von 32,8(43) Jahren und einer gemittelten Sprunganzahl von 2468(1678) Sprüngen.

${ }^{101}$ Werte der Eigenschaftsangst größer als 29 und $\leq 33$ auf der Trait-Skala des STAI. 


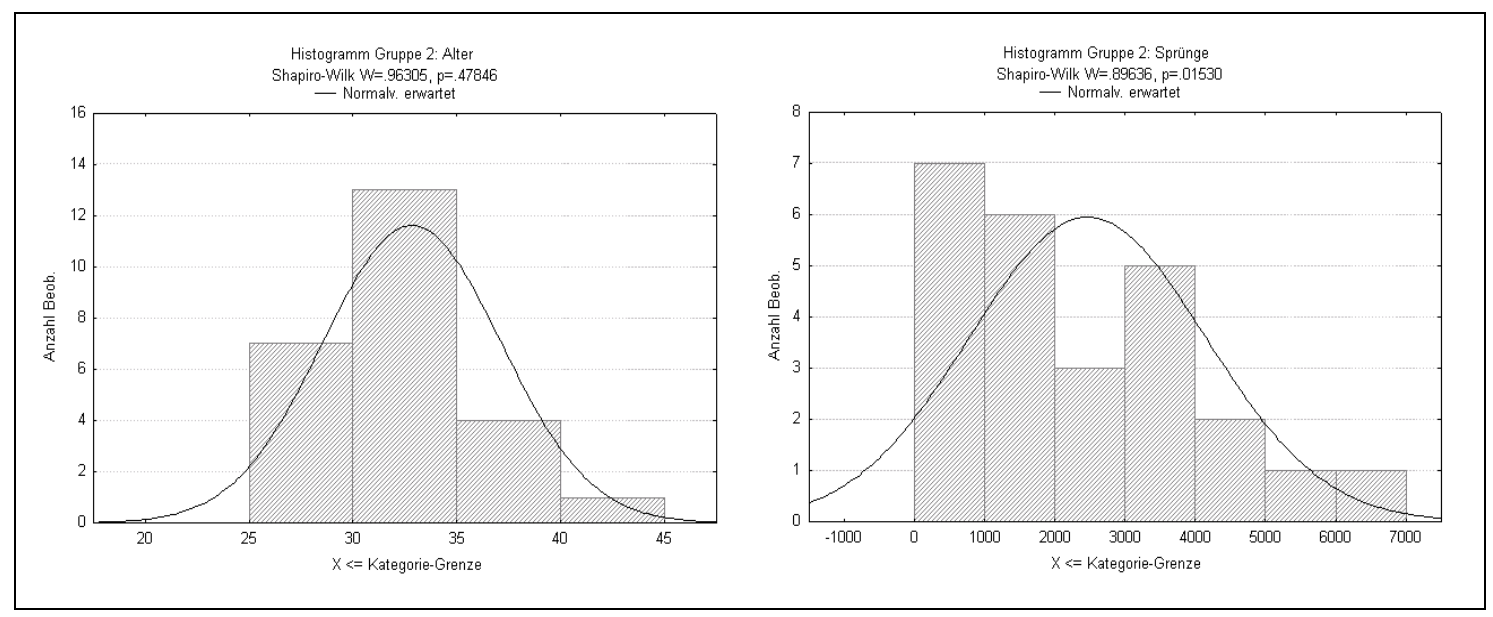

Abbildung 22: Histogramme von Alter und Sprungerfahrung der Gruppe 2 mit mittlerer Eigenschaftsangst.

Tabelle 8: Die Probanden der Gruppe 3: Hohe Ängstlichkeit.

\begin{tabular}{|c|c|c|c|c|c|c|}
\hline $\begin{array}{c}\text { Ängstlichkeits- } \\
\text { gruppe }\end{array}$ & Proband & $\mathbf{m} / \mathbf{w}$ & Alter & Disziplin & $\begin{array}{l}\text { Sprung- } \\
\text { erfahrung }\end{array}$ & $\begin{array}{c}\text { Sprünge im } \\
\text { Rahmen der } \\
\text { Untersuchungen } \\
\end{array}$ \\
\hline \multirow{31}{*}{ 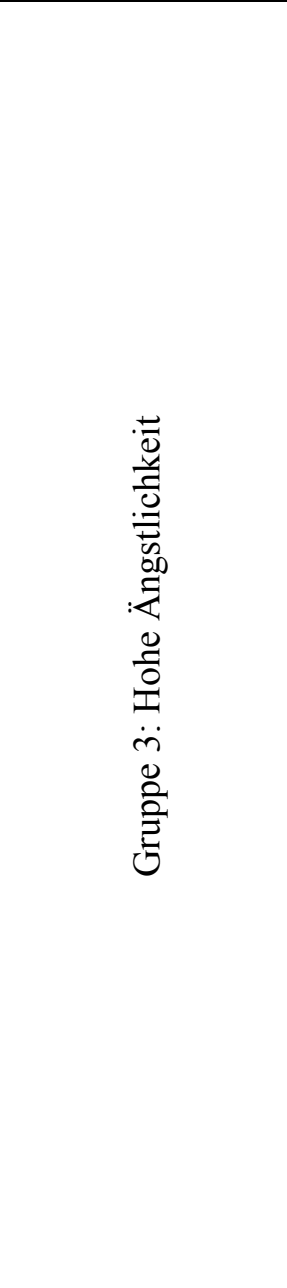 } & AnM-Fear4-D & $\overline{\mathrm{w}}$ & 19 & $\overline{4}$ & 290 & 2 \\
\hline & AnK-Airf4-D & $\mathrm{w}$ & 24 & 4 & 300 & 2 \\
\hline & NiZ-CYP4-EN & $\mathrm{m}$ & 36 & 4 & 325 & 1 \\
\hline & EiR-Last4-D & $\mathrm{w}$ & 28 & 4 & 370 & 2 \\
\hline & ChS-Blau4-D & $\mathrm{w}$ & 35 & 4 & 400 & 3 \\
\hline & ClG-Schn4-D & $\mathrm{w}$ & 33 & 4 & 530 & 2 \\
\hline & DaK-Airf4-D & $\mathrm{w}$ & 26 & 4 & 570 & 2 \\
\hline & SuF-Heho8-D & $\mathrm{w}$ & 20 & 8 & 670 & 2 \\
\hline & RaH-PhSc8-D & $\mathrm{m}$ & 29 & $4 / 8$ & 700 & 4 \\
\hline & SoS-Blau4-D & $\mathrm{w}$ & 32 & 4 & 715 & 1 \\
\hline & SaH-Shak4-D & $\mathrm{w}$ & 29 & 4 & 860 & 3 \\
\hline & ElH-Teamo8-D & $\mathrm{m}$ & 42 & 8 & 970 & 3 \\
\hline & JeM-Silv4-D & $\mathrm{m}$ & 34 & 4 & 1200 & 4 \\
\hline & JoB-Jwm8-D & $\mathrm{m}$ & 31 & 8 & 1300 & 2 \\
\hline & CoK-Schn4-D & $\mathrm{w}$ & 38 & 4 & 1350 & 2 \\
\hline & RoB-Cros4-D & $\mathrm{m}$ & 31 & 4 & 1370 & 1 \\
\hline & MaK-Grou4-D & $\mathrm{w}$ & 34 & 4 & 1500 & 2 \\
\hline & SiS-Jwm8-D & $\mathrm{w}$ & 31 & 8 & 1700 & 2 \\
\hline & MaB-Team4-D & $\mathrm{m}$ & 39 & 4 & 1700 & 2 \\
\hline & BaL-Teamo8-D & $\mathrm{w}$ & 39 & 8 & 1930 & 2 \\
\hline & GuG-Ms4/8-D & $\mathrm{m}$ & 34 & $4 / 8$ & 2000 & 4 \\
\hline & MoT-Eigh8-D & $\mathrm{w}$ & 37 & $4 / 8$ & 2400 & 2 \\
\hline & BiB-Team4-D & $\mathrm{w}$ & 42 & 4 & 2500 & 2 \\
\hline & JuK-Der4-D & $\mathrm{m}$ & 40 & $4 / 8$ & 2600 & 3 \\
\hline & LuG-Fris4-D & $\mathrm{m}$ & 44 & 4 & 3100 & 2 \\
\hline & YoK-JAP8-EN & $\mathrm{w}$ & 34 & 8 & 3500 & 3 \\
\hline & WoV-LO8-D & $\mathrm{m}$ & 40 & 8 & 3910 & 2 \\
\hline & UwS-Xray4-D & $\mathrm{m}$ & 40 & 4 & 4100 & 5 \\
\hline & PiP-A4-D & $\mathrm{m}$ & 47 & 4 & 4800 & 1 \\
\hline & ToK-BW4-D & $\mathrm{m}$ & 26 & 4 & 5000 & 2 \\
\hline & MaW-GER8-D & $\mathrm{m}$ & 37 & 8 & 6100 & 4 \\
\hline
\end{tabular}


Die dritte Gruppe mit Probanden einer hohen Eigenschaftsangst ${ }^{102}$ setzt sich aus 16 Frauen und 15 Männern mit einem Durchschnittsalter von 33,9(67) Jahren und einer Sprungerfahrung von 1896(1565) Sprüngen zusammen.

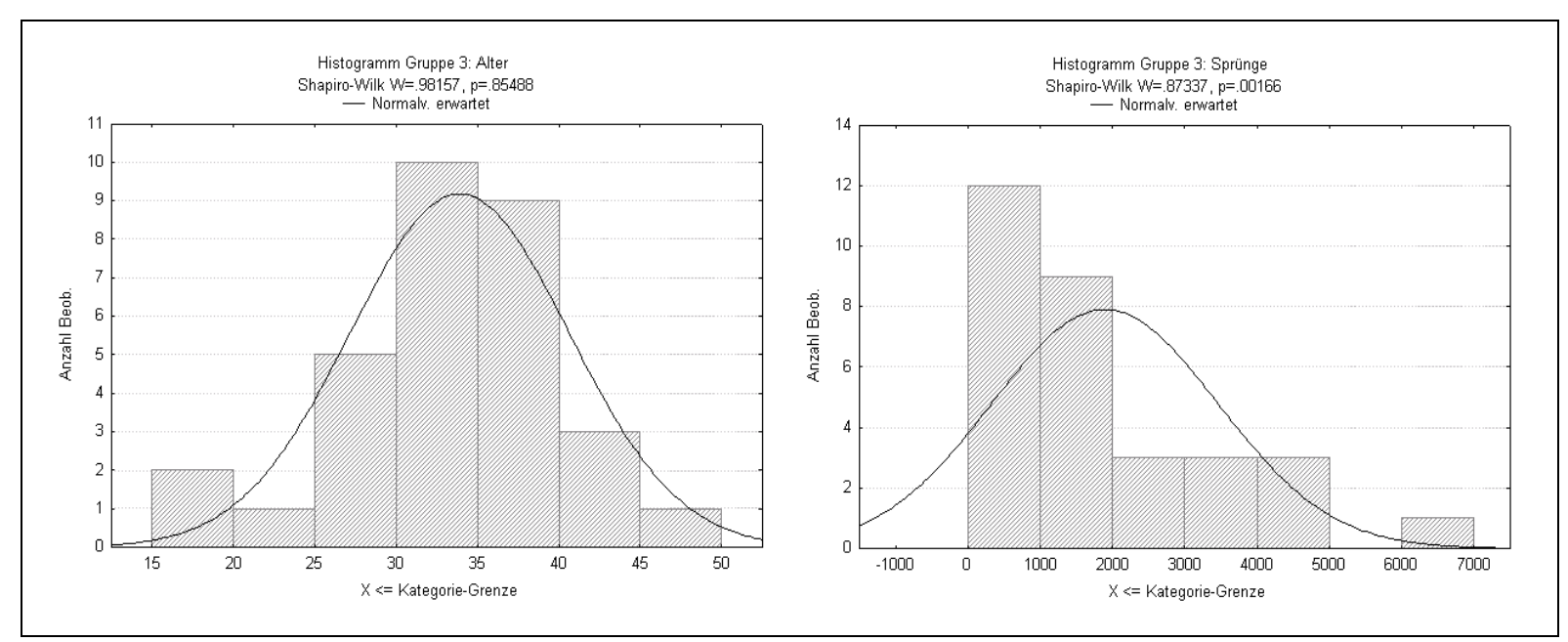

Abbildung 23: Histogramme von Alter und Sprungerfahrung der Gruppe 3 mit hoher Eigenschaftsangst.

Die Aufteilung der drei Gruppen anhand der Eigenschaftsangst ist recht homogen: Die Standardabweichungen des Alters betragen 20,6\%, 13,1\% bzw. 19,8\% der jeweiligen Mittelwerte. Die Standardabweichungen der Sprungerfahrungen liegen mit 96,6\%, 68,0\% und $82,5 \%$ eher etwas weiter auseinander. Die Prüfung auf Normalverteilung nach SHAPIROWILKS ergibt allerdings, dass die Zusammensetzung der Gruppen nur in zwei Fällen statistisch signifikant einer Normalverteilung entspricht. Da die Messgrößen Alter und Sprungerfahrung jedoch nur deskriptiven Charakter im Rahmen der Untersuchungen haben und nicht weiter betrachtet werden, ergeben sich aus dieser Nicht-Normalverteilung keine Konsequenzen.

\subsection{Untersuchungsverlauf}

Alle Probanden wurden vor den untersuchten Sprüngen mit einem POLAR „Accurex Plus“ Herzfrequenzmessgerät ausgestattet, das während eines gesamten Sprungablaufs alle fünf Sekunden die Herzfrequenz der Testperson abspeicherte. Jeweils zum Aufzeichnungsbeginn wurden die Messgeräte mit einer Stoppuhr synchronisiert, um die Herzfrequenz zu den

\footnotetext{
${ }^{102}$ Werte größer oder gleich 34 auf der Trait-Skala des STAI.
} 
genauen Zeiten für den Start der Absetzmaschine, den ca. 15minütigen Steigflug bis zur Sprunghöhe, die Absprung-, Schirmöffnungs- und Landungsphase zu erhalten. Zusätzlich wurden die Probanden angewiesen, jeweils beim Start des Flugzeugs, beim „Run-In“-Signal ca. eine Minute vor dem Absprung und nach erfolgter Fallschirmöffnung eine Markierungstaste an den POLAR-Geräten zu betätigen.

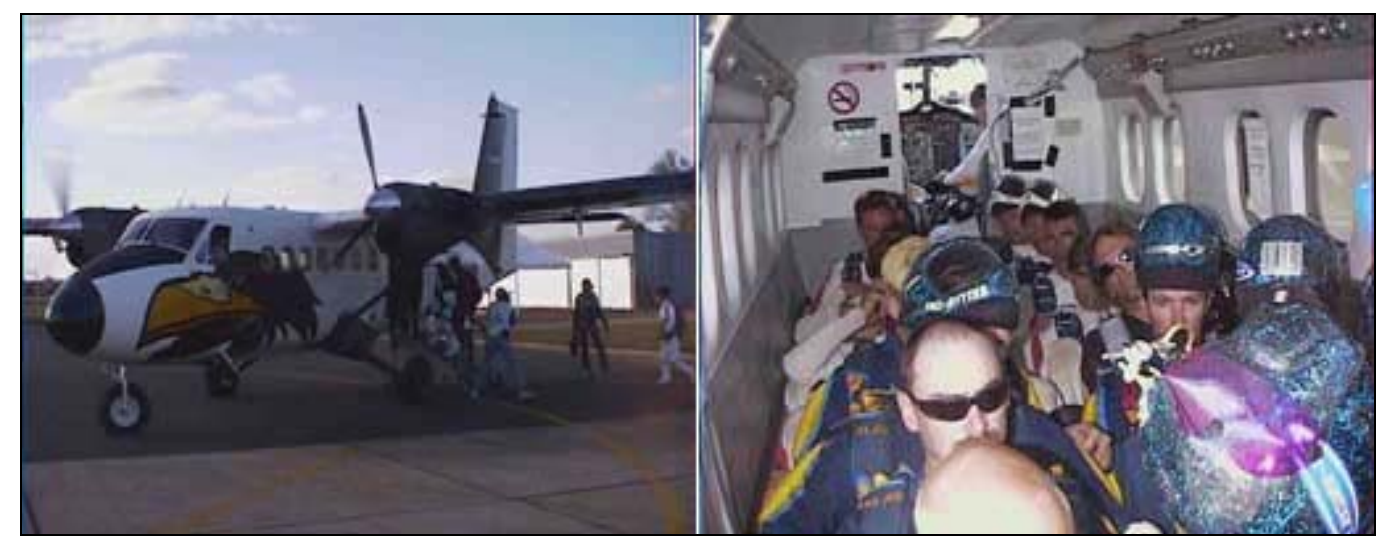

Abbildung 24: Absetzflugzeug DHC-6 „, Twin Otter" während der WM 1999 von außen und innen (Foto: Ted Wagner).

Sämtliche Sprünge wurden aus Absetzflugzeugen vom Typ DeHavilland DHC-6 „Twin Otter“ durchgeführt, da alle im Rahmen der Untersuchungen beobachteten Wettbewerbe ausschließlich diesen Flugzeugtyp nutzten. Dabei wurden jeweils entweder zwei Achtermannschaften auf 4000m bzw. vier Viererteams auf 3200m Höhe über Grund gebracht. Die Wettkämpfer saßen in dem Flugzeug aus Platzgründen auf dem Boden - erst ca. 500m vor Erreichen der Absprunghöhe ist ein gebücktes Stehen zur Vorbereitung auf den Absprung möglich.

Nach dem Ablegen der Sprungausrüstung ${ }^{103}$ erhielten die Testpersonen einen CSAI-2 Fragebogen nach MARTENS zur Messung der Zustandsangstkomponenten mit der Anweisung, diesen Fragebogen jeweils nach den Gefühlen zu den Zeitpunkten „Start des Flugzeugs“, „Eine Minute vor dem Absprung“ und „Im Freifall“ zu beantworten. Anhand der Fragebogenskalen wurden dann die kognitive und somatische Angstkomponente sowie die Selbstwirksamkeitsüberzeugung ermittelt.

Zur Ermittlung der Leistungswerte erhielt jeder Proband die Aufgabe, seine persönliche im Sprung erbrachte Leistung auf einer freien Skala von 0\%-100\% zu bewerten. Für den

${ }^{103}$ Etwa 2 bis maximal 5 Minuten nach der Landung. 
Untersuchungsteil während der Weltmeisterschaften 1999 wurde dabei eine deutsche, englische, spanische, russische und norwegische Fassung bzw. Übersetzung des CSAI-2 verwendet; die Untersuchungsteile der Deutschen Meisterschaften 2000 und 2002 nutzten nur die deutsche Version. Die letzte Stelle der Probandenkürzel gibt jeweils Auskunft über die Sprache der verwendeten Fragebögen ${ }^{104}$ - der Großteil der Probanden nutzte die deutsche bzw. englische Originalfassung des CSAI-2 und STAI.

Die Herzfrequenzwerte wurden mittels eines POLAR „Interface Plus“ in den Computer eingelesen. In einem 30-Sekunden-Intervall um die drei obigen Zeiten des Fragebogens wurden Durchschnittswerte $\Delta H F$ errechnet.

Um diese Werte besser zwischen den Probanden vergleichen zu können, wurden die Durchschnittswerte mittels Ruhepuls $H F_{\text {Ruhe }}$ und maximaler Herzfrequenz ${ }^{105} H F_{\text {Maximal }}$ auf eine standardisierte Skala der physiologischen Aktivierung umgerechnet. Diese Skala reicht von $0 \%$ bis $100 \%$ bzw. von 0 bis 1 .

Gleichung 5: Berechnung der physiologischen Aktivierung.

$$
\Delta \text { physioaktiv }=\frac{\Delta H F-H F_{\text {Ruhe }}}{H F_{\text {Maximal }}-H F_{\text {Ruhe }}}
$$

Somit existierte für jeden beobachteten Sprung eines Probanden jeweils zu drei Zeiten eine Kombination der Messwerte von kognitiver und somatischer Angstkomponente, physiologischer Aktivierung und Selbstwirksamkeit, zusätzlich die Note der Selbstbeurteilung der erbrachten Leistung im Sprung.

\footnotetext{
${ }^{104} \mathrm{Vgl}$. Anm. 98 auf Seite 93.

105 Angenähert durch die Formel $H F_{\text {Maximal }}=220-$ Lebensalter (nach FoX et al. 1971).
} 


\section{Kritik an der Methodik}

Die Untersuchungen im Rahmen der vorliegenden Arbeit konnten an internationalen Versuchspersonen während dreier wichtiger Wettkämpfe durchgeführt werden - dies stellte eine einmalige Chance dar, eine hoch professionelle Probandengruppe unter Wettbewerbsstress $\mathrm{zu}$ untersuchen. Dabei wurden die Untersuchungen so geplant, dass sie die Wettkämpfer möglichst wenig ablenkten, denn sämtliche Daten wurden parallel während des Wettkampfablaufes gewonnen. Sicherlich waren hierfür geringfügige Einschränkungen (z.B. hinsichtlich des Zeitpunktes der Fragebogenerhebungen) vonnöten, die sich kritisieren lassen, andererseits gelang es im Rahmen der Untersuchungen aber auch, einige der Schwächen und Kritikpunkte vorhergehender Untersuchungen im Fallschirmsport $\mathrm{zu}$ vermeiden. Im Folgenden wird auf mögliche Kritikpunkte an Planung und Durchführung dieser Untersuchungen eingegangen.

\subsection{Systematische Aspekte}

Einer der häufigsten Kritikpunkte an vorherigen Untersuchungen im Fallschirmsport ist die schlechte Vergleichbarkeit von Sprungsituationen, die dadurch entstanden, dass Anfänger mit fortgeschrittenen Fallschirmspringern verglichen werden sollten. Hierbei wurden in der Regel aber sehr unterschiedliche Aufgaben durchgeführt: Die Anfänger führten Sprünge mit automatischer Auslösung aus niedrigen Höhen ${ }^{106}$ durch, die Fortgeschrittenen Freifallsprünge aus größeren Höhen ${ }^{107}$ (vgl. Fenz/EPSTEIN 1967, 36; Schedlowski/Tewes 1992, 97). Hieraus resultierten gravierende Unterschiede hinsichtlich der Aufgabenanforderung und physiologischer Reaktionen (vgl. RoTH et al. 1996, 64).

Durch die ausschließliche Betrachtung von Freifallformationsspringern im Wettkampf konnten diese Kritikpunkte vermieden werden. Selbst die beobachteten Trainingssprünge fanden bereits am Ort des Wettkampfes und unter Wettkampfregeln statt. Dadurch ist eine identische sportliche Aufgabenstellung vorhanden und die Absprunghöhe ist einheitlich auf 3200m bzw. 4000m (jeweils $\pm 50 \mathrm{~m}$ ) über Grund festgelegt. Die Absprunghöhen der beiden Disziplinen liegen recht dicht beieinander; im Rahmen einer physiologischen Höhenakklimatisation wird eine signifikante Erhöhung der Herzfrequenz erst bei Höhen oberhalb

\footnotetext{
106 Jeweils ca. $1000 \mathrm{~m}$ über Grund.

${ }^{107}$ Bei FENZ/EPSTEIN 1500-5000m, bei SCHEDLOWSKI/TEWES 2500-3500m über Grund.
} 
von 4000-4500m vorausgesagt (vgl. DE MARÉEs 1992, 233f; HollmanN/HetTINGER 2000, 461f). Bei Sprunghöhen zwischen 3000 und 4000m konnte in Untersuchungen keine Erhöhung der Herzfrequenz als kurzfristige Höhenakklimatisation nachgewiesen werden (vgl. REID et al 1971, 1205f; SCHEDLOWSKI/ TEWES 1992, 97; RoTH et al. 1996, 71).

Zwar fanden die Untersuchungen dieser Arbeit an drei verschiedenen Orten - sogar auf zwei verschiedenen Kontinenten - statt, allerdings waren die absoluten Höhen der Sprungplätze über dem Meeresspiegel (Corowa: 143m ü. NN, Gera 309m ü. NN, Gransee: 50m ü. NN) und die klimatischen Bedingungen jeweils sehr ähnlich, sodass keine physiologischen Unterschiede zwischen den drei Messorten auftreten sollten.

Da in allen Untersuchungsteilen das gleiche Absetzflugzeug ${ }^{108}$ verwendet wurde, sind weiterhin keine Unterschiede hinsichtlich des verschiedenen Kraftaufwands z.B. für das Herausklettern aus dem Flugzeug für den Absprung zu erwarten. Durch den engen Platz im Flugzeug sind großräumige Bewegungen der Sportler nahezu ausgeschlossen. Somit eignet sich die Herzfrequenz hier in besonderem Maße zur Messung der Erregung - dabei ist allerdings stets zu beachten, dass die Herzfrequenz auch durch positive Emotionen wie z.B. Freude erhöht werden könnte (vgl. Abschnitt 2.2.1 auf Seite 20).

In einigen Fällen zeigte sich das Messgerät, der POLAR „Accurex Plus“, als vermutlich nicht kompatibel mit den Funkanlagen der Flugzeuge. So besteht der Herzfrequenzmesser aus einem um die Brust auf der Haut getragenen Messband, das per Funk die Signale zu einer Armbanduhr und Speichereinheit überträgt. In einigen wenigen Fällen wurden für kurze Zeiträume entweder Nullwerte oder physiologisch nicht sinnvolle Werte ${ }^{109}$ gemessen. Diese Probleme traten nur bei wenigen Testgeräten auf, stets aber in Phasen, in denen der Pilot mit seinen Funkgeräten sendete.

Weil aus organisatorischen Gründen eine direkte Bestimmung der maximalen Herzfrequenz nicht möglich war, wurde diese abgeschätzt. In der Literatur gibt es einige Formeln zur Schätzung der maximalen Herzfrequenz. Die geläufigste und vor allem im Breitensport angewendete Formel 220 - Lebensalter (nach FOX et al. 1971) wurde auch bei diesen Untersuchungen herangezogen. Jüngste Untersuchungen von ENGELS et al. (1998) haben ergeben, dass die mittlere Abweichung von gemessener und mit der Formel 220 - Lebensalter geschätzter maximaler Herzfrequenz mit dem Alter zunimmt. Die in diesen Untersuchungen

\footnotetext{
${ }^{108}$ Und da die Fluggeschwindigkeit beim Absetzvorgang in den Wettbewerbsregeln ebenfalls vorgeschrieben ist.

${ }^{109}$ Eine konstant hohe Herzfrequenz über 210 ohne Schwankungen.
} 
betrachtete Probandengruppe im Alter zwischen 19 und 58 Jahren weist eine Differenz zwischen tatsächlicher und geschätzter maximaler Herzfrequenz kleiner als 5\% auf (ENGELS et al. 1998, 97). Somit ist trotz der Nutzung eines Schätzwertes nur mit geringen Genauigkeitsverlusten bei der Betrachtung der Herzfrequenz zu rechnen, zumal die Ermittlung der persönlichen physiologischen Aktivierung interindividuelle Differenzen der Probanden ausgleicht.

$\mathrm{Zu}$ beachten ist jedoch, dass die verwendete Schätzformel nur für eine rein körperliche Belastung gültig ist. In der konkreten Situation des Fallschirmsprungs wird die Herzfrequenz sowohl durch die Angstreaktion und damit verbundene Hormonausschüttung ins Blut und durch die körperliche Belastungskomponente erhöht - beide Größen bewirken über den Sympathikus ein Ansteigen der Herzfrequenz (vgl. HollmanN/HetTinger 2000, 83).

Die Herzfrequenz stellt leider die einzige objektive bzw. unabhängig gemessene Größe der Untersuchungen dar. Alle anderen Messdaten der Untersuchungen beziehen sich auf Fragebogendaten. Das Untersuchungsergebnis ist somit in besonderem Maße von der Ehrlichkeit der Probanden beim Ausfüllen der Fragebögen abhängig. Zwar wurde der CSAI-2 zusammen mit den „Anti-Social Desirability Instructions“ (vgl. MARTENS et al. 1990, 52) ausgegeben, um die Probanden zu motivieren, Angst als etwas Positives zu sehen (vgl. Abschnitt 2.2.2 auf Seite 21). Ob diese Instruktionen jedoch ihren Zweck erfüllt haben und ob der Fallschirmspringer als Persönlichkeitstyp nicht von jeher dazu neigt, sich selbst als ,nicht ängstlich“ einzustufen, bleibt abzuwarten.

Ähnliches gilt auch für die Leistungsbewertung, die jeder Proband selbst durchführte. Wieder erscheint hier der Punkt der Ehrlichkeit des Probanden bei seiner Leistungsbeurteilung von Bedeutung. Dazu kommt noch ein Kritikpunkt, der aus einer Untersuchung von KREMPEL (1985) folgt. Hier wurde nachgewiesen, dass Stabhochspringer in bis zu 40\% aller Fälle nicht in der Lage waren, ihre eigene Leistung in Übereinstimmung mit einer externen Beobachtung einzuschätzen (vgl. KREMPEL 1985, 153 zit. nach ZIMMERMANN 2001, 51).

Sicherlich wäre also eine externe Beurteilung der erbrachten Leitung im Sprung wünschenswert gewesen, mit einfachen Mitteln ist dies jedoch nicht $\mathrm{zu}$ realisieren. Das offizielle Punkteergebnis eines Sprunges ist zwar einfach zu erhalten, jedoch handelt es sich um eine Mannschaftsleistung. Die gute oder schlechte Leistung des Einzelnen kann sich in der Mannschaftsleistung wiederspiegeln, kann jedoch aber auch von Mannschaftsmitgliedern „aufgefangen“ oder überdeckt werden. Eine externe Beobachtung und Leistungsbeurteilung 
kann so nur auf den Videobändern des Sprunges erfolgen, die jedoch nicht immer zugänglich sind. Ferner würde sich die Frage nach leistungsbeschreibenden Beobachtungsfaktoren stellen, will man die Videoauswertung als objektive Methode zur Leistungsmessung eines einzelnen Sportlers einsetzen.

Somit wird erwartet, dass die Selbsteinschätzung der Probanden, Ehrlichkeit vorausgesetzt, die Fehler bei der Leitungsmessung minimiert. Die Skala der Leistung (0\%-100\% frei wählbar) ist jedenfalls fein genug, um Leistungsdifferenzen feststellen zu können. So kritisiert z.B. JONES, dass viele Untersuchungen bei der Leistungsmessung nur zwischen Erfolg/ Misserfolg, Sieg/Niederlage oder guter/mittlerer/schlechter Leistung unterschieden und somit Differenzen nicht genau genug darstellen konnten ${ }^{110}$ (vgl. JONES 1995, 461).

COHEN et al. (2003) fordern eine differentialpsychologische Herangehensweise zum Testen eines Katastrophenmodells von Angst und sportlicher Leistung (vgl. COHEN et al. 2003, 146). Ich gehe aber davon aus, dass die Bildung von Probandengruppen ähnlicher Ängstlichkeit einen gelungenen Kompromiss zwischen der Betrachtung der Gesamtgruppe und einem differentialpsychologischen Ansatz darstellt - zusätzlich wurde so schneller eine hinreichende Datenmenge von Sprüngen unter Wettbewerbsbedingungen erreicht.

Zur Zusammenstellung der Probandengruppen wurde die Ängstlichkeit mit der Trait-Skala des STAI-Fragebogens nach SPIELBERGER gemessen. Hier ist zu beachten, dass die Gruppeneinteilungen zwar anhand von niedriger, mittlerer und hoher Eigenschaftsangst durchgeführt wurden, es sich hierbei aber ausdrücklich um relative Werte $^{111}$ handelt. Der STAI-Mittelwert der Eigenschaftsangst beträgt in der Gruppe der Hochängstlichen nur 39,1(54) auf einer Skala mit Werten zwischen 20 und 80 und entspricht damit lediglich einer prozentualen Ängstlichkeit von 31,8\%. Dies ist in einer Betrachtung absoluter Werte der Eigenschaftsangst kein Wert, bei dem eine Klassifizierung als Hochängstlicher gerechtfertigt wäre.

Betrachtet man jedoch das Kriterium der langjährigen Ausübung des Fallschirmsports als Selektionsmechanismus oder Filter, bei dem von vornherein hochängstliche Persönlichkeiten eine Ausbildung abbrechen ${ }^{112}$, so ist das Vorhandensein der relativ niedrigen STAI-Werte in dieser Untersuchung ${ }^{113}$ verständlich (vgl. EIDE/ATTERÅs 1978, 102f; BREIVIK 1999, 11). Bei der Probandengruppe handelt es sich um Fallschirmspringer, die im Mittel diesen Sport seit

\footnotetext{
${ }^{110}$ Vgl. Anm. 26 auf Seite 29.

${ }^{111}$ Gemessen an der Zusammensetzung der (fallschirmspringenden) Probandengruppe.

${ }^{112}$ Oder gar nicht erst eine Ausbildung zum Fallschirmspringer beginnen.

${ }^{113}$ STAI-Mittelwert aller 88 Probanden: 32,4(65). Dies entspricht einer prozentualen Ängstlichkeit von 20,7\%.
} 
10,8(58) Jahren ausüben. Folglich wäre anzunehmen, das in einer solchen Gruppe keine Persönlichkeiten hoher Eigenschaftsangst $\mathrm{zu}$ finden sind. Eine Untersuchung von BREIVIK (1999) zu Persönlichkeitsmerkmalen von sogenannten Risikosportlern belegt diese These: Erfahrene Fallschirmspringer zeichnen sich unter anderem durch niedrigere Werte der Eigenschaftsangst aus (vgl. BREIVIK 1999, 21f). Somit lässt sich das Auftreten der geringen STAI-Werte in dieser Untersuchung durch den Filter-Effekt der fortwährenden Ausübung des Fallschirmsports erklären ${ }^{114}$.

Weiterhin stellt die internationale Zusammensetzung der Probandengruppe sicherlich einen großen Vorteil dar, wenn man wirklich hochgradig professionelle Fallschirmspringer untersuchen will. Jedoch stellt sich die Frage, ob Angst innerhalb verschiedener Kulturen nicht unterschiedlich erlebt bzw. interpretiert wird (vgl. ANSHEL et al. 1997, 145).

Solche kulturabhängig verschiedenen Auslegungsweisen des Angsterlebens oder -verarbeitens könnten das Untersuchungsergebnis erheblich verfälschen. Allerdings ist zu beachten, dass derartige internationale Unterschiede hauptsächlich im Rahmen der Angstbewältigung aufgeführt werden. Somit behielten die erhobenen Messdaten von momentanen Angstwerten $^{115}$ und sportlicher Leistung ihre Gültigkeit. Gegebenenfalls wären nur unterschiedliche Schlussfolgerungen hinsichtlich der praktischen Ergebnisrelevanz und der Umsetzung für ein Training mit „optimaler“ Coping-Strategie aus den Untersuchungsergebnissen der internationalen Probanden zu erwarten.

Einen weiteren Kritikaspekt stellen in diesem Zusammenhang der internationalen Probandengruppe auch die verschiedenen Sprachen dar; dieser Gesichtspunkt wird im Hinblick auf die in der Untersuchung verwendeten Fragebögen im nächsten Abschnitt dargestellt.

Letzter systematischer Kritikpunkt ist die Tatsache, dass die Angst nicht synchron zur Leistungssituation gemessen werden konnte. Gerade bei der Kürze der Anforderungsdauer beim Freifallformationsspringen finden alle leistungsentscheidenden Ereignisse in Sekundenbruchteilen statt. In Anbetracht der Tatsache, dass innerhalb dieses so kurzen Zeitintervalls Hormonausschüttungen stattfinden, die ein Selbsterleben u.U. verfälschen, können viele Erlebnisse bereits vergessen oder beschönigt sein, was für die Angstmessung nach der

\footnotetext{
${ }^{114}$ Dieser Filtereffekt scheint beim Fallschirmspringen besonders früh einzusetzen: Etwa 85\% aller Teilnehmer von Erstspringerkursen führen ihre Ausbildung nicht weiter fort (vgl. BREIVIK 1999, 11).

115 Diese Angstwerte sind ja als stetiges Endergebnis oder „Output“ des dynamischen Prozesses von Angsterleben und Angstbewältigung anzusehen.
} 
Situation problematisch erscheint. So erhielt DURTSCHI (2001) in Angst-Leistungs-Messungen die zuverlässigsten Werte, wenn die Angst direkt während der Wettkampfsituation ermittelt wurde (vgl. DURTSCHI 2001, 198).

Andererseits zeigte die Meta-Analyse von KLEINE und SchwARZER (1991), dass sich eine engere Angst-Leistungs-Beziehung ergibt, wenn man die Angst nach und nicht vor der Leistungsprüfung misst (vgl. KLEINE/SCHWARZER 1991, 19). Weiterhin ist die Ablenkung der Probanden im Wettkampf natürlich geringer, wenn die Angstmessung erst nach dem Fallschirmsprung erfolgt. Fraglich bleibt aber nach wie vor, ob es zulässig ist, Messdaten zu mehreren Zeiten zu erheben und hiervon ausgehend Aussagen auf genau einen Zeitpunkt bezogen zu machen, ohne dabei große Fehler zu verursachen (vgl. JONES 1995, 469).

Eine Betrachtung des Vorgehens anderer Untersuchungen zeigt allerdings, dass die in der vorliegenden Studie gewählte Herangehensweise der Datenerhebung mitten in einem Wettbewerb bereits eine Besonderheit darstellt. Nur wenige weitere Untersuchungen konnten ebenfalls Daten parallel zum Wettkampfgeschehen erheben (vgl. KJøRMO/HALVARI 2002, 956; PARfitT/PATES 1999, 353; Sharma et al. 1994, 1147; TAYLOR 1987, 145).

In etwa der Hälfte der von mir betrachteten Untersuchungen wird zwar der Zusammenhang von Wettkampfangst und sportlicher Leistung untersucht, die Messungen fanden aber als „Laborexperimente“ abseits der Wettkampfsituation ${ }^{116}$ statt und verglichen Messdaten der Probanden vor und nach dem Wettkampf miteinander (vgl. Jerome/WiLLIAMS 2000, 241; RAUDSEPP/KAIS 2002, 406; GRASSO 2000, 12; IDZIKOWSKi/BADDELEY 1987, 1465; MiLLS 1992, 22; EDWARDS/HARDY 1996, 299f). Vor diesem Hintergrund ist die zeitliche Verschiebung bei der Erfassung der Messdaten in den vorliegenden Untersuchungen als geringfügig anzusehen.

Abschließend sei angemerkt, dass der Zeitpunkt der Angstmessung in den von mir durchgeführten Untersuchungen variierte: Die CSAI-2-Fragebögen wurden zwar direkt nach der Rückkehr zum Aufenthaltsbereich der Springer ausgefüllt, durch die verschiedenen Freifallzeiten in der 4er- und 8er-Disziplin und die unterschiedlichen Flugzeiten am Fallschirm ${ }^{117}$ lagen die Zeitpunkte der Angstmessung jedoch in einem Intervall von 3,5 bis 9 Minuten nach dem Absprung. Im Vergleich zu anderen Untersuchungen scheint diese Zeitspanne gering,

\footnotetext{
${ }^{116}$ In der Regel wurde jeweils am Beginn und Ende eines Wettkampftages eine solche „Extrauntersuchung“ durchgeführt - gelegentlich fand die Voruntersuchung sogar bereits am Tag vor dem Wettkampf statt.

117 Die Flugzeit, also die Summe von der Freifallzeit und der Zeit am geöffneten Fallschirm, betrug bei allen beobachteten Sprüngen im Mittel 158(28) Sekunden (Min.: 100s, Max.: 295s).
} 
gemessen an der Kürze des freien Falls sollte dies jedoch näher betrachtet werden. Dazu wurden die Probanden anhand der zwischen Absprung und Landung verstrichenen Zeit in je eine Gruppe kurzer ${ }^{118}$ und langer ${ }^{119}$ Gesamtflugzeit eingeteilt.

Ein t-Test für unabhängige Stichproben ergab zunächst, dass die Gesamtflugzeit in der 4erDisziplin hoch signifikant kürzer als in der 8er-Disziplin ist (4er: 152s; 8er: 168s; $p=0,000$ ). Die Differenz von etwa 16s entspricht genau der Freifallzeit, die für das Durchfallen der um 800m höheren Absprunghöhe in der 8er-Disziplin benötigt wird.

Hinsichtlich der gemessenen Herzfrequenzen, der physiologischen Aktivierung, der mit dem CSAI-2 ermittelten kognitiven Zustandsangstkomponente und der Selbstwirksamkeitsüberzeugung lassen sich zwischen den Gruppen kurzer und langer Gesamtflugzeit keine signifikanten Mittelwertunterschiede nachweisen. Einzig die somatische Angstkomponente ist bei der Gruppe langer Gesamtflugzeit signifikant größer als bei der Gruppe kurzer Gesamtflugzeit (lang: 0,35; kurz: $-0,15 ; p=0,019$ ). Dieser signifikante Mittelwertunterschied der somatischen Zustandsangstkomponente betrifft allerdings nur die beiden Gruppen extremer Gesamtflugzeiten: Vergleicht man diese beiden Gruppen mit den restlichen beobachteten Sprüngen mittlerer ${ }^{120}$ Flugzeit, lassen sich keine signifikanten Unterschiede bei allen obigen Größen feststellen. Da die somatische Angstkomponente zudem in den von mir angepassten Katastrophenmodellen von Angst und sportlicher Leistung kombiniert mit der physiologischen Aktivierung eingesetzt wird, kann man davon ausgehen, dass sich aus den unterschiedlichen Gesamtflugzeiten und den daraus resultierenden Variationen der Zeiten bis zum Ausfüllen der Fragebögen keine Verfälschungen ergeben.

\subsection{Kritik an den verwendeten Fragebögen}

Systematische Aspekte, wie die Ehrlichkeit des Probanden beim Ausfüllen eines Fragebogens, wurden bereits im vorigen Abschnitt angesprochen, es bleibt generelle Kritik an den verwendeten Fragebögen. Sowohl der STAI-Fragebogen von SPIELBERGER als auch der CSAI-2 von MARTENS sind in der sportwissenschaftlichen Literatur anerkannt. Ihre Validität wurde mehrfach überprüft; inzwischen ist der CSAI-2 eines der am weitesten verbreiteten und

\footnotetext{
${ }^{118}$ Zeit zwischen Absprung und Landung < 140 Sekunden ( 55 beobachtete Sprünge).

${ }^{119}$ Zeit zwischen Absprung und Landung > 175 Sekunden (53 beobachtete Sprünge).

${ }^{120}$ Zeit zwischen Absprung und Landung $\geq 140$ und $\leq 175$ Sekunden ( 84 beobachtete Sprünge).
} 
am häufigsten genutzten Instrumente zur Selbstbeschreibung der Angst in der Sportpsychologie (vgl. PARFITT et al. 2000, 72; PAIN 1999, 1).

Zumindest der STAI ist auch auf Unterschiede hinsichtlich kulturabhängiger Beantwortungen überprüft worden - mit der Bestätigung einer kulturunabhängigen Tauglichkeit (vgl. Morgan/ElLiCKSON 1989, 170; LAUX et al. 1981, 32f).

In der Literatur konnten vom STAI nur die englische Originalfassung von SPIELBERGER sowie die deutsche Übersetzung von LAUX et al., vom CSAI-2 nur die englische Originalfassung gefunden werden. Es stellte sich die Frage, ob nun alle Probanden nur den Fragebogen in einer der beiden Sprachen erhalten und für sich selbst zur Beantwortung übersetzen müssen oder ob die Variante der Übersetzung der Fragebögen in andere Sprachen auftretende Fehler minimieren kann.

Da ein Fragebogen in besonderem Maße sprachliche Mittel zur Angstmessung nutzt, kommt natürlich eventuellen Übersetzungsfehlern eine spezielle Bedeutung zu. Daher wurden die Fragebögen vor Beginn der Untersuchung von mehreren Experten pro Sprache in ausgewählte Sprachen übersetzt. Auf diese Art und Weise konnten fragebogenspezifische Formulierungen richtig in die jeweiligen Sprachen übernommen werden.

Insgesamt wurden in den Untersuchungen 179 deutschsprachige, 29 englische, 4 norwegische, 2 spanische und 2 Fragebögen in russischer Sprache verwendet. Die russischen Fragebögen konnten allerdings wegen fehlender Werte in der Gesamtauswertung der Untersuchung nicht berücksichtigt werden ${ }^{121}$.

Eine Varianzanalyse ${ }^{122}$ der STAI- und CSAI-2-Fragebogenwerte in den vier Gruppen unterschiedlicher Fragebogensprachen ergibt keine signifikanten Abweichungen innerhalb der Subskalen von Eigenschaftsangst (STAI), kognitiver und somatischer Angstkomponente und Selbstwirksamkeitsüberzeugung (CSAI-2). Zusätzlich zeigt auch ein Vergleich der Normalverteilungen der CSAI-2-Subskalen keine nennenswerten Unterschiede zwischen deutscher und englischer CSAI-2-Fassung ${ }^{123}$. Somit ist die Validität der verwendeten Fragebögen hinreichend nachgewiesen.

\footnotetext{
121 Bei einem der russischen Probanden waren zu viele Fragen des CSAI-2 ausgelassen, bei dem anderen versagte die Herzfrequenzmessung.

${ }^{122}$ Einfaktorielle ANOVA mit nachfolgendem SCHEFFÉ-Test.

123 Bei dem Vergleich der Normalverteilungen bzw. der einzelnen Datenpunkte wurden aufgrund einer zu geringen Datenmenge die norwegische und spanische CSAI-2-Übersetzung nicht mehr betrachtet, zumal die vorherige Varianzanalyse bereits keine Differenzen hinsichtlich der Fragebogensprache aufzeigte.
} 
Die Normalverteilungen von STAI und den einzelnen CSAI-2-Subskalen sind in Abbildung 25 und Abbildung 26 graphisch dargestellt.

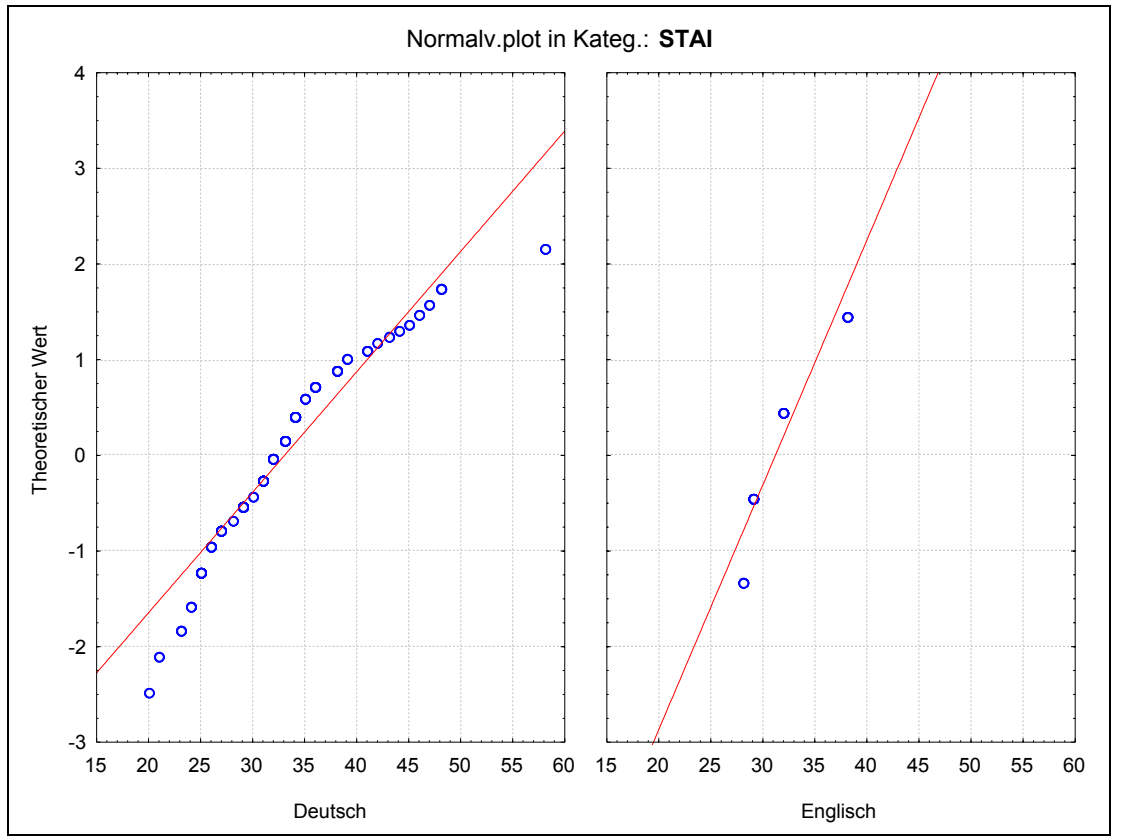

Abbildung 25: Normalverteilungsplot: STAI in deutscher und englischer Fassung.

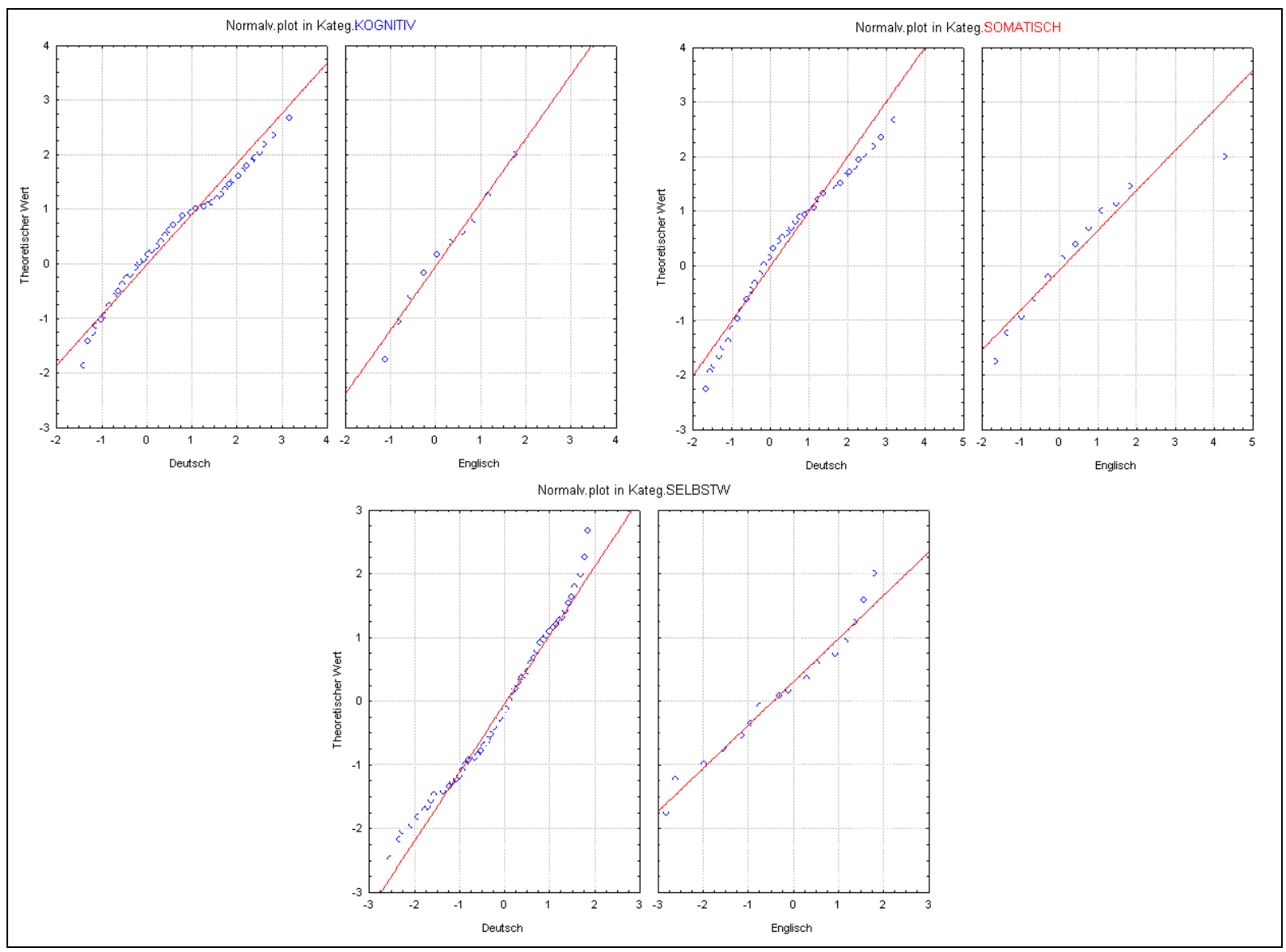

Abbildung 26: Normalverteilungsplot: CSAI-2 in deutscher und englischer Fassung. 


\section{Untersuchungsergebnisse}

Alle aus den CSAI-2-Fragebögen gewonnenen Daten sowie die Leistungsnoten wurden zur statistischen Auswertung anhand Gleichung 6 z-transformiert.

Gleichung 6: Berechnung der standardisierten Werte z aus Mittelwert und Standardabweichung.

$$
z_{i}=\frac{x_{i}-\bar{x}}{\sigma}
$$

Dabei sind die $x_{i}$ die einzelnen Fragebogenwerte, $\bar{x}$ der Mittelwert sowie $\sigma$ die Standardabweichung der jeweiligen Werte (vgl. WILLIMCZIK 1997, 62). Alle so standardisierten Werte wurden mit den Programmen STATISTICA, DATAFIT und GEMCAT II weiterbearbeitet und ausgewertet.

\subsection{Deskriptive Ergebnisse}

Zunächst werden die wichtigsten gemessenen Daten dargestellt. Dies sind die gemessenen Herzfrequenzen und die umgerechneten physiologischen Aktivierungen sowie die Daten aus den Fragebögen.

\subsubsection{Verlauf der Herzfrequenz}

Die gemessenen Herzfrequenzen zeigten einheitlich bei allen Springern einen typischen Verlauf, wie er unter anderem von Rотн et al. (1996) beschrieben wird. In Abbildung 27 und Abbildung 28 auf der folgenden Seite ist dieser Verlauf in den Disziplinen der 4er- und der 8er-Formation dargestellt. Diese Unterscheidung erfolgte, da aufgrund der etwas niedrigeren Absprunghöhe im Vierer der Steigflug mit dem Flugzeug und die Freifallphase kürzer ausfallen. Der Steigflug dauerte in der 4er-Disziplin im Durchschnitt 13 (8er: 17) Minuten, die Freifallphase ca. 45 (8er: 60) Sekunden.

Man beachte jedoch, dass der Verlauf der Herzfrequenzen in den beiden Disziplinen ansonsten fast identisch ist - die Unterscheidung in den Abbildungen erfolgte nur wegen der unterschiedlichen zeitlichen Stauchung der Messkurven. 


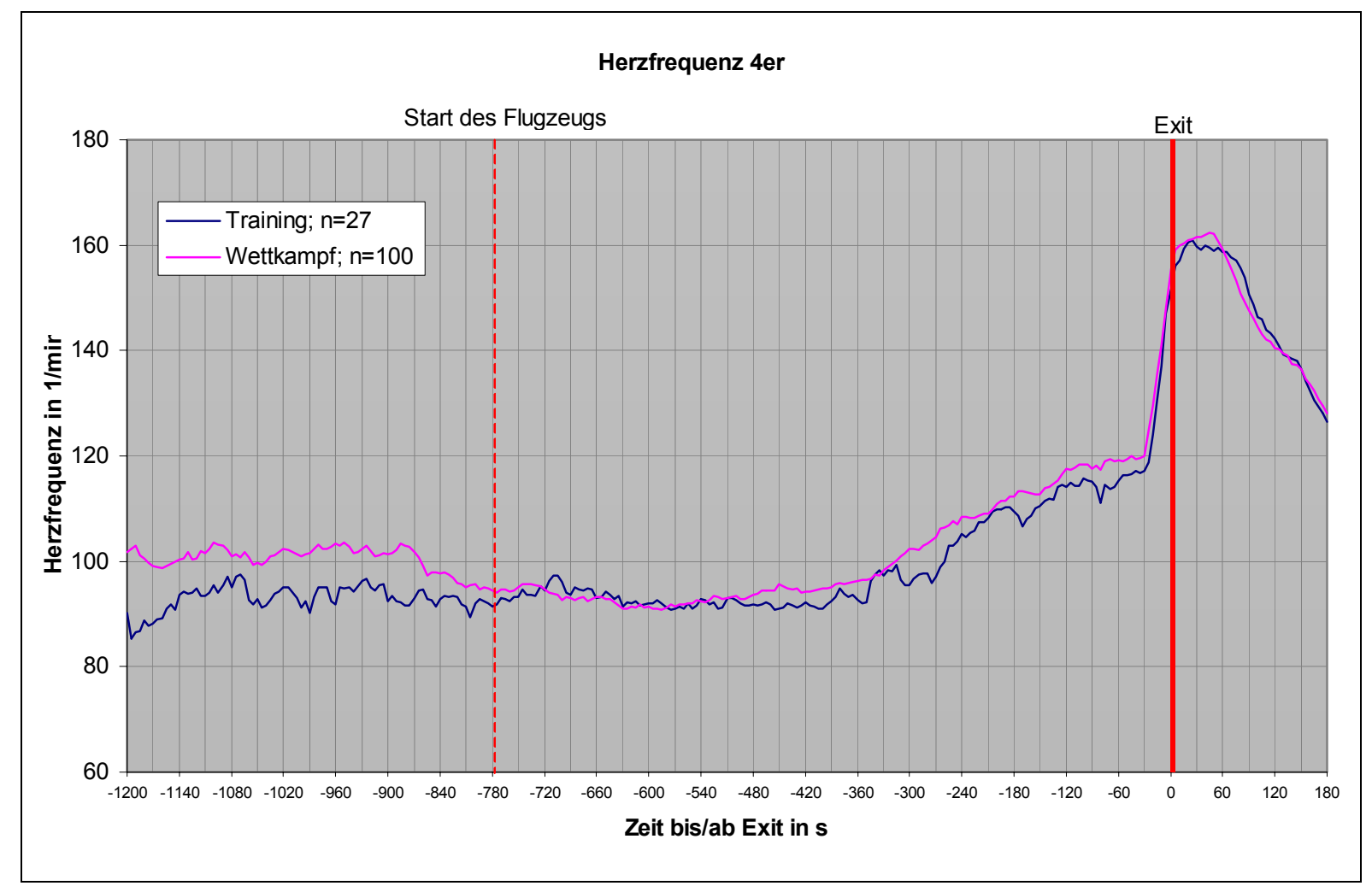

Abbildung 27: Durchschnittliche Herzfrequenz der 4er-Formationen in Training und Wettkampf.

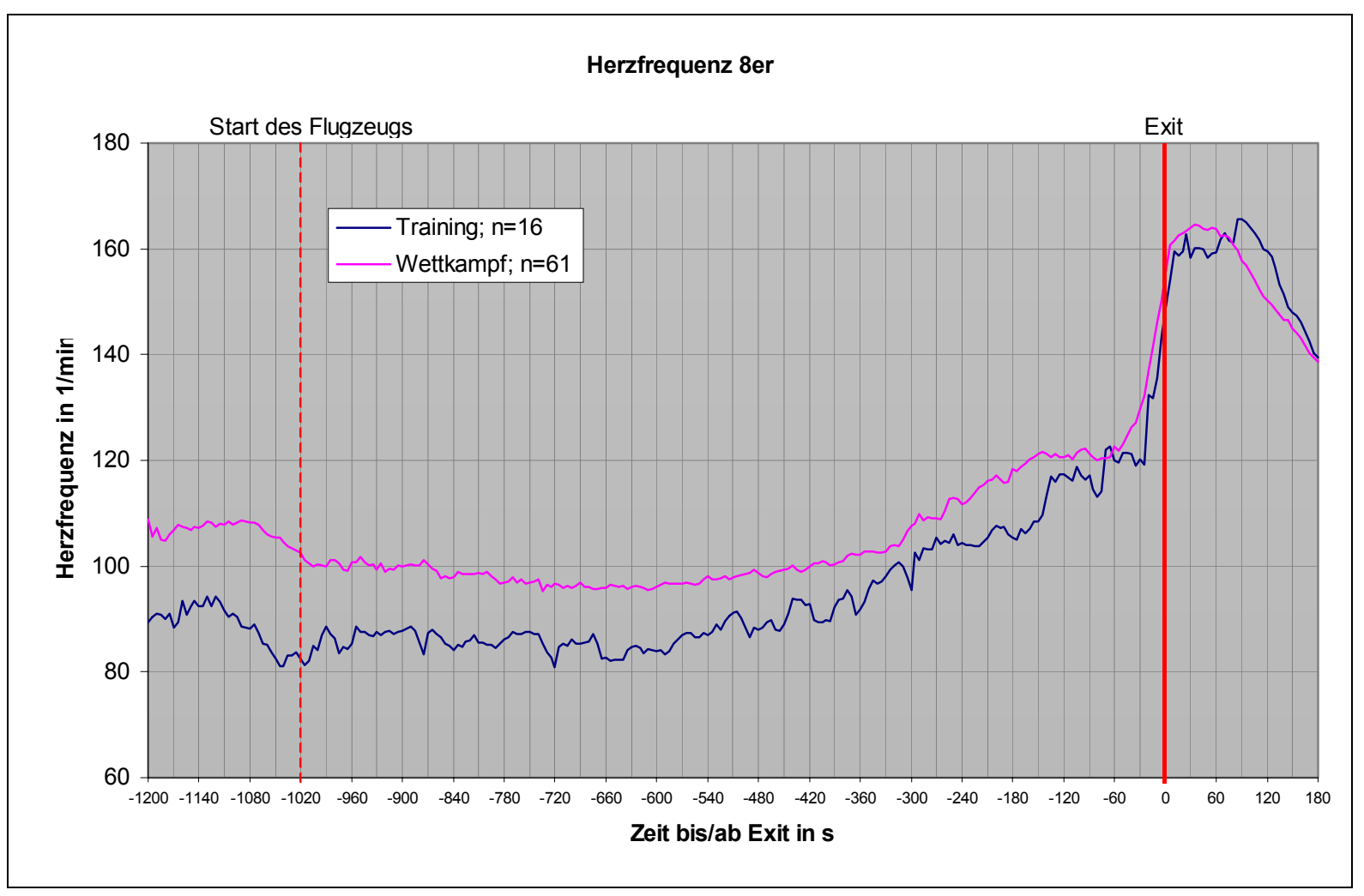

Abbildung 28: Durchschnittliche Herzfrequenz der 8er-Formationen in Training und Wettkampf. 
Man erkennt, dass die Herzfrequenz während des Sitzens im Flugzeug zunächst mit durchschnittlich 94 Schlägen pro Minute recht niedrig ist. Ungefähr fünf Minuten vor Erreichen der Absprunghöhe erkennt man eine Zunahme der Herzfrequenz, wenn sich die Springer fertig machen. Im Moment des Absprungs steigt die Herzfrequenz sprunghaft an und erreicht in der Freifallphase (in der Regel kurz vor dem Öffnen des Fallschirms) ihren höchsten Wert von durchschnittlich 161(13) Schlägen pro Minute (Minimum: 109; Maximum: 196). Dieser Wert liegt um etwa 20 Schläge pro Minute über der Herzfrequenz, die SÜss/MuthNy (2002) in einer Meta-Analyse von 21 Studien an erfahrenen Fallschirmspringern als typische Herzfrequenz während der Freifallphase ermittelten (vgl. MUTHNY/ Süss 2003, 242f). Diese Differenz der Herzfrequenzwerte lässt sich vermutlich durch die physische Belastungskomponente der in den vorliegenden Untersuchungen ausschließlich betrachteten Freifallformationsspringer begründen.

Während der Flugphase mit dem Fallschirm, die im Durchschnitt 1,7(5) Minuten dauerte ${ }^{124}$, sinkt die Herzfrequenz deutlich auf Werte in der Größenordnung von 140 Schlägen pro Minute ab, um dann kurz vor der Landung eine kleine, aber erkennbare, Spitze zu zeigen. Weil die Sportler Fallschirme unterschiedlicher Größe und Flugcharakteristik verwendeten und da die Flugzeit am geöffneten Fallschirm in erheblichem Maße von den geflogenen Manövern abhängt ${ }^{125}$, variierten die Zeiten von der Fallschirmöffnung bis zur Landung bei den Probanden sehr stark ${ }^{126}$. Da in Abbildung 27 und Abbildung 28 jedoch Durchschnittswerte der Herzfrequenz aller Probanden aufgetragen wurden, ist aufgrund dieser unterschiedlichen Flugzeiten am Fallschirm die Spitze nicht immer zu erkennen, wohl aber in den individuellen Verläufen der Herzfrequenzen. Abbildung 29 auf der folgenden Seite zeigt exemplarisch die Herzfrequenz eines Probanden, der einen Trainingssprung ${ }^{127}$ und zwei Wettkampfsprünge innerhalb der 4er-Disziplin absolvierte.

\footnotetext{
${ }^{124}$ In der 4er- und der 8er-Disziplin sind zwar Sprunghöhen und Freifallzeiten unterschiedlich - da aber alle Fallschirmspringer in ca. 800m über Grund ihren Fallschirm öffnen, sind in etwa identische Flugzeiten am Fallschirm zu erwarten.

${ }^{125}$ Der Fallschirm wird umso schneller an Höhe verlieren, je mehr steile Kurven geflogen werden.

${ }^{126}$ Die Flugzeiten am geöffneten Fallschirm betrugen zwischen 0,8 und 4,0 Minuten.

${ }^{127}$ Bei dem Trainingssprung in Abbildung 29 ist zu beachten, dass dieser nicht aus 3200m, sondern aus 4000m Höhe absolviert wurde. Aufgrund der etwas längeren Freifallzeit ist daher der Verlauf der Herzfrequenz bei dem Trainingssprung gegenüber den anderen Sprüngen ab dem Moment der Schirmöffnung geringfügig verschoben.
} 


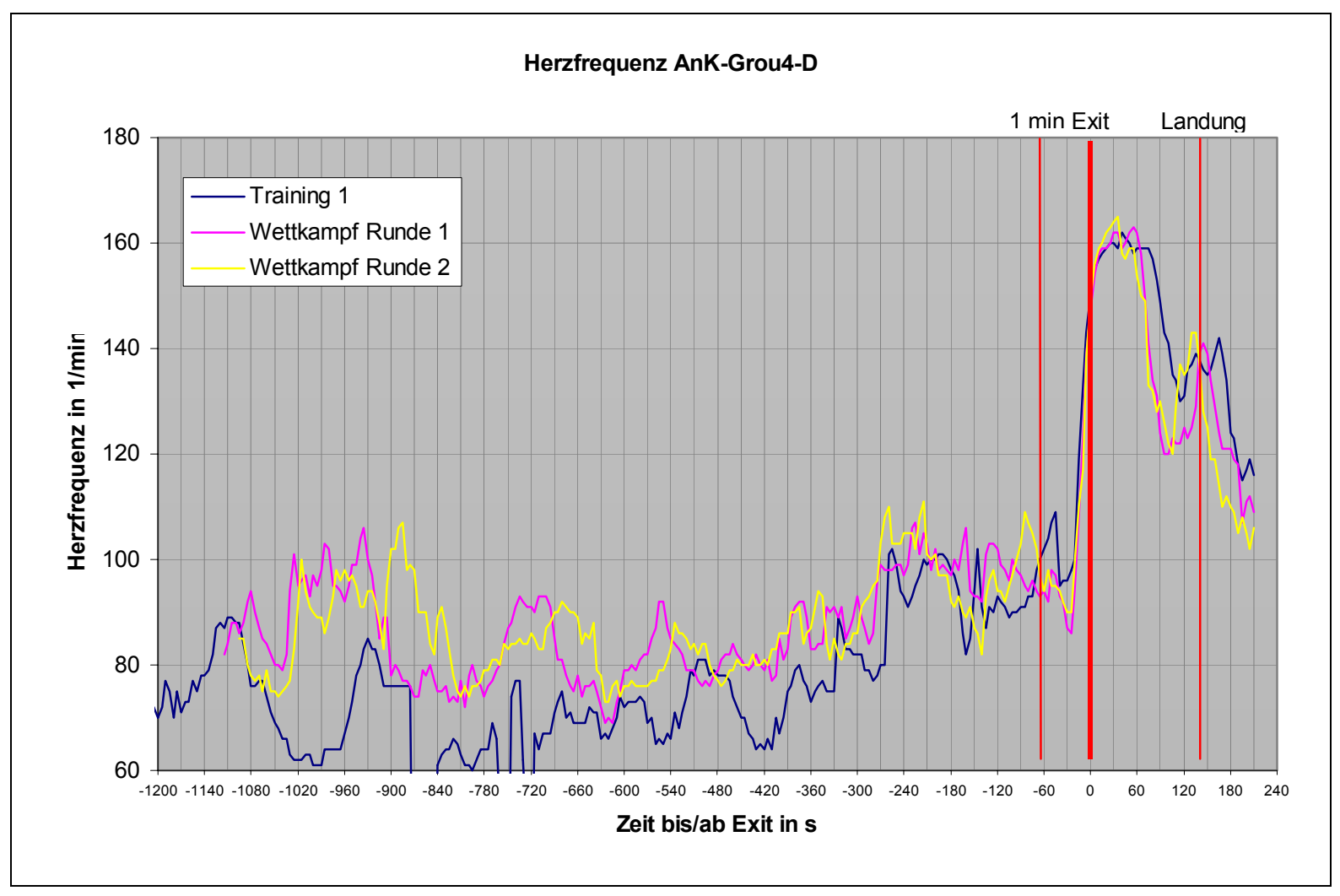

Abbildung 29: Herzfrequenz des Probanden AnK-Grou4-D in Training und Wettkampf.

Eine Betrachtung der gemittelten Herzfrequenzwerte aus Abbildung 27 und Abbildung 28 zeigt weiterhin, dass in beiden Disziplinen die Herzfrequenzen im Wettkampf über denen des Trainings liegen - in der 8er-Disziplin ist dieser Effekt etwas stärker ausgeprägt.

Die Durchschnittswerte der errechneten physiologischen Aktivierung, wiederum aufgeteilt nach der 4er- und der 8er-Disziplin, ergeben einen identischen Kurvenverlauf wie der der Herzfrequenz. Daher wird auf eine graphische Darstellung dieser Größe verzichtet.

$\mathrm{Zu}$ beachten ist jedoch, dass die Werte der physiologischen Aktivierung eine wesentlich größere Streuung aufweisen, da sie physiologische Unterschiede der Probanden hinsichtlich Geschlecht, Trainingszustand, Alter etc. indirekt berücksichtigen ${ }^{128}$. So beträgt in der 4erDisziplin die durchschnittliche Herzfrequenz während des erfassten Sprungzeitraums im Training 105(16), im Wettkampf 108(16) Schläge pro Minute; in der 8er-Disziplin im Training 103(13) und im Wettkampf 112(15) Schläge pro Minute. Die Standardabweichungen betragen hier 15\% (bzw. 15\%, 13\% und 14\%) der jeweiligen Mittelwerte. Betrachtet man die

\footnotetext{
${ }^{128}$ Alle diese Faktoren beeinflussen Ruhepuls und maximale Herzfrequenz eines Probanden.
} 
physiologische Aktivierung, so betragen die Standardabweichungen 38\% (bzw. 36\%, 41\% und 34\%) der jeweiligen Mittelwerte ${ }^{129}$.

Diese größere Streuung der Messwerte der physiologischen Aktivierung könnte eine bessere Untersuchung des Angst-Leistungs-Zusammenhangs ermöglichen, da eine größere Bandbreite an Werten angenommen wird. Solch eine große Bandbreite ist wünschenswert; ansonsten würden womöglich so enge Wertebereiche betrachtet, dass der untersuchte Zusammenhang nicht vollständig oder nur ausschnittweise zu erkennen ist (vgl. IDZIKOWSKI/BADDELEY 1987, 1472).

\subsubsection{Selbsteinschätzungen der Probanden}

Da von allen Angstkomponenten des CSAI-2 jeweils Werte zu drei verschiedenen Zeiten existierten, wurden von den jeweiligen Werten zunächst Mittelwerte gebildet. Die physiologische Aktivierung wurde für jeden Probanden über ein 30-Sekunden-Intervall der jeweiligen Zeiten (Start des Flugzeugs, 1 Minute vor Absprung, Freifall) errechnet. Auf eine vollständige Auflistung aller Fragebogendaten wird aufgrund der großen Datenmenge an dieser Stelle verzichtet; stattdessen folgen in tabellarischer Form die Durchschnittswerte der drei Ängstlichkeitsgruppen.

Tabelle 9: Mittelwerte und Standardabweichungen der Ängstlichkeitsgruppen:

Fragebogenwerte und physiologische Aktivierung.

\begin{tabular}{|c|c|c|c|c|c|}
\hline & $\begin{array}{c}\text { Kognitive } \\
\text { Angst }\end{array}$ & $\begin{array}{c}\text { Somatische } \\
\text { Angst }\end{array}$ & $\begin{array}{c}\text { Physiologische } \\
\text { Aktivierung }\end{array}$ & $\begin{array}{c}\text { Selbstwirksamkeits- } \\
\text { überzeugung }\end{array}$ & Leistung \\
\hline $\begin{array}{c}\text { Gruppe 1: } \\
\text { Niedrige } \\
\text { Angstlichkeit }\end{array}$ & $-0,45(86)$ & $-0,21(91)$ & $0,56(9)$ & $0,13(112)$ & $-0,15(110)$ \\
\hline $\begin{array}{c}\text { Gruppe 2: } \\
\text { Mittlere } \\
\text { Ängstlichkeit }\end{array}$ & $-0,20(74)$ & $-0,09(105)$ & $0,58(10)$ & $0,20(88)$ & $0,15(102)$ \\
\hline $\begin{array}{c}\text { Gruppe 3: } \\
\text { Hohe } \\
\text { Ängtlichkeit }\end{array}$ & $0,64(102)$ & $0,29(101)$ & $0,60(10)$ & $-0,30(90)$ & $-0,02(87)$ \\
\hline
\end{tabular}

\footnotetext{
${ }^{129}$ Für die physiologische Aktivierung erhält man die folgenden Durchschnittswerte und Standardabweichungen: 4er Training: 0,34(11); 4er Wettkampf: 0,39(13); 8er Training 0,33(11); 8er Wettkampf 0,40(12).
} 
Diese Daten sind in Abbildung 30 in einem Interaktionsplot dargestellt. Man erkennt, dass mit zunehmender Ängstlichkeit die Mittelwerte von kognitiver und somatischer Angstkomponente zunehmen, ebenso der Durchschnittswert der physiologischen Aktivierung ${ }^{130}$. Der Mittelwert der Selbstwirksamkeitsüberzeugung nimmt bei wachsender Eigenschaftsangst tendenziell ab, der Durchschnittswert der Leistung schwankt.

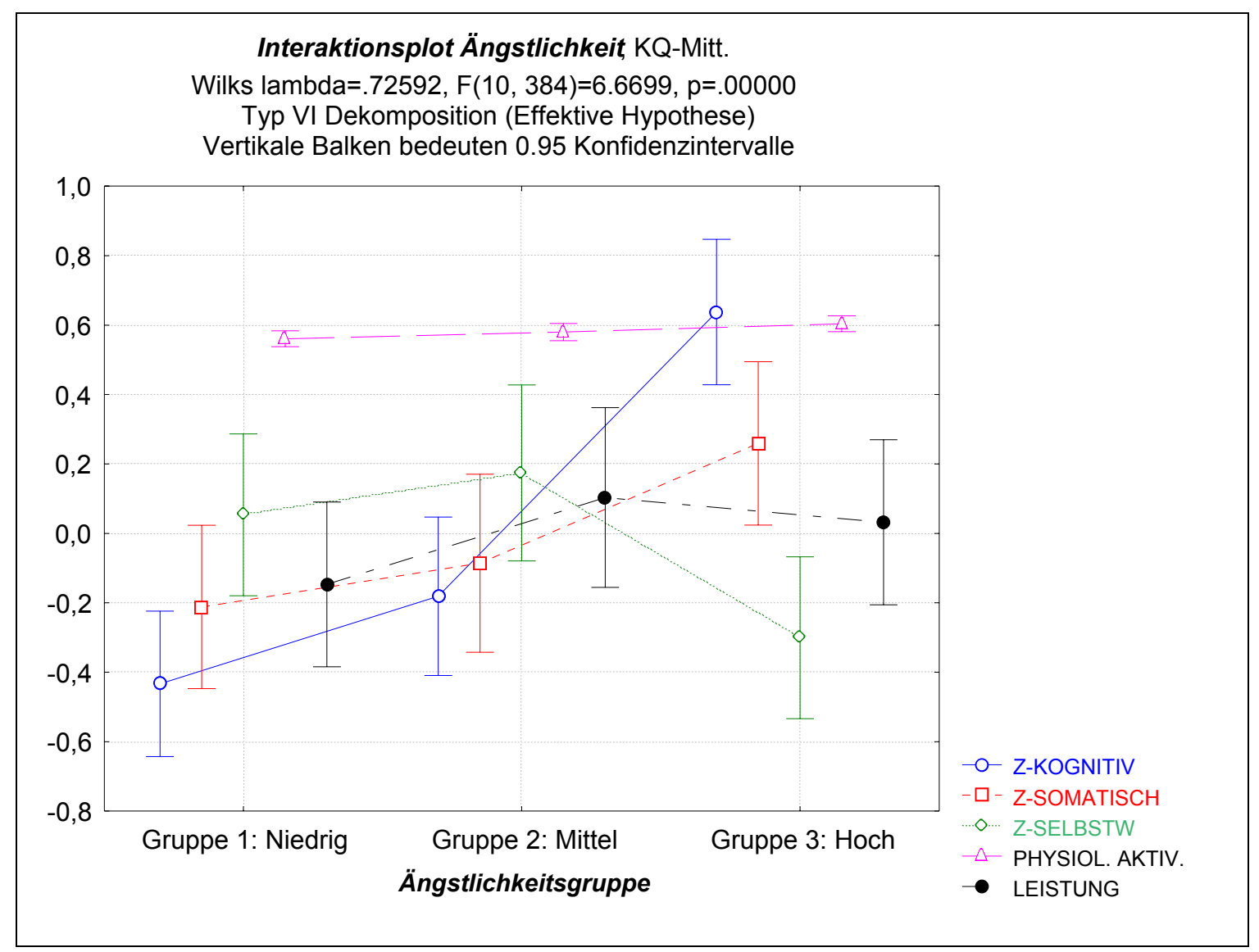

Abbildung 30: Interaktionsplot der Ängstlichkeitsgruppen: Fragebogenwerte und physiologische Aktivierung.

An dieser Stelle sei aber auf die recht hohen Standardabweichungen der Werte in Tabelle 9 hingewiesen. So zeigt der Interaktionsplot in Abbildung 30 zwar recht plausibel erscheinende Zusammenhänge, jedoch muss die Signifikanz dieser Aussagen erst noch überprüft werden. Siehe hierzu Abschnitt 5.2.2 zur Hypothesenprüfung.

Da der Selbstwirksamkeitsüberzeugung im Katastrophenmodell des Angst-Leistungs-Zusammenhangs eine besondere Rolle zugeschrieben wird (siehe Abschnitt 4.3), erfolgte eine neue Aufteilung der Datensätze anhand der Stärke der Selbstwirksamkeitsüberzeugung: Z-Werte

${ }^{130}$ Allerdings nur geringfügig. 
der Selbstwirksamkeit kleiner als $-0,5$ bildeten die erste Gruppe niedriger Selbstwirksamkeitsüberzeugung (57 Datensätze), Werte zwischen $-0,5$ und 0,5 die Gruppe mittlerer (83 Datensätze) und z-Werte größer als 0,5 die Gruppe hoher Selbstwirksamkeit (74 Datensätze). Die Mittelwerte und Standardabweichungen der Angstwerte und physiologischen Aktivierung sind in Tabelle 10 und als Interaktionsplot in Abbildung 31 dargestellt.

Tabelle 10: Mittelwerte und Standardabweichungen der Selbstwirksamkeitsgruppen:

Fragebogenwerte und physiologische Aktivierung.

\begin{tabular}{|c|c|c|c|c|}
\hline & $\begin{array}{c}\text { Kognitive } \\
\text { Angst }\end{array}$ & $\begin{array}{c}\text { Somatische } \\
\text { Angst }\end{array}$ & $\begin{array}{c}\text { Physiologische } \\
\text { Aktivierung }\end{array}$ & Leistung \\
\hline $\begin{array}{c}\text { Z-SELBSTW<-0,5: } \\
\text { Niedrige } \\
\text { Selbstwirksamkeits- } \\
\text { überzeugung }\end{array}$ & $0,81(109)$ & $0,60(119)$ & $0,59(9)$ & $-0,33(85)$ \\
\hline $\begin{array}{c}\mathbf{- 0 , 5} \text {-Z-SELBSTW<0,5: } \\
\text { Mittlere } \\
\text { Selbstwirksamkeits- } \\
\text { überzeugung }\end{array}$ & $-0,01(73)$ & $-0,05(82)$ & $0,60(10)$ & $0,03(99)$ \\
\hline $\begin{array}{c}\mathbf{0 , 5}<\text { Z-SELBSTW: } \\
\text { Hohe }\end{array}$ & $-0,61(73)$ & $-0,41(80)$ & $0,55(9)$ & $0,23(107)$ \\
$\begin{array}{c}\text { Selbstwirksamkeits- } \\
\text { überzeugung }\end{array}$ & & & \\
\hline
\end{tabular}

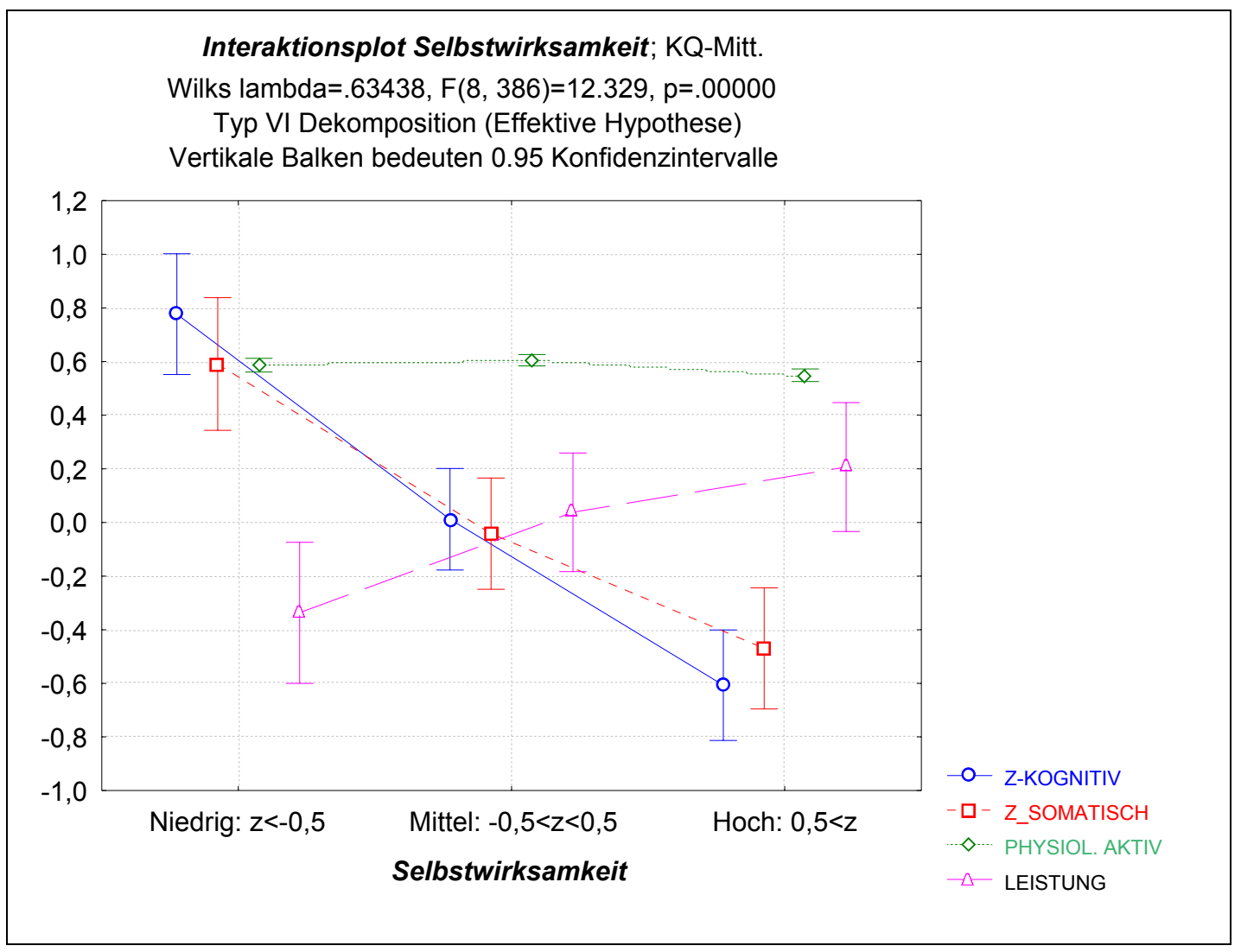

Abbildung 31: Interaktionsplot der Selbstwirksamkeitsgruppen:

Fragebogenwerte und physiologische Aktivierung. 
Man erkennt in Tabelle 10 und Abbildung 31, dass für steigende Werte der Selbstwirksamkeitsüberzeugung die kognitive und die somatische Angstkomponente deutlich, die physiologische Aktivierung geringfügig, abnimmt. Weiterhin wächst die im Sprung erbrachte Leistung.

Dieses Verhalten scheint die positiven Effekte der Selbstwirksamkeit innerhalb des AngstLeistungs-Zusammenhanges zu bestätigen. Da die Standardabweichungen aber wiederum sehr groß sind, handelt es sich hier auch nur um qualitative Vermutungen, deren Signifikanz später geprüft werden muss.

Abschließend wurden die Probanden anhand ihres individuellen Erfahrungsstandes im Freifallformationsspringen in drei Erfahrungsgruppen eingeteilt. Die Springer mit weniger als 1000 Formationssprüngen und weniger als 5 Jahren Erfahrung im Fallschirmsport ${ }^{131}$ bildeten die Gruppe der (relativ) unerfahrenen Formationsspringer. Probanden, die zwischen 1000 und 2500 Formationssprüngen absolviert hatten und zwischen 5 und 10 Jahren Erfahrung im Fallschirmsport aufwiesen, bildeten eine Gruppe mittleren Erfahrungsstandes - Springer mit mehr als 2500 Formationssprüngen und mehr als 10 Jahren Erfahrung im Sport die Gruppe der hoch erfahrenen Fallschirmspringer. Im Zweifelsfall entschied hier die Zahl der Sprünge in der Spezialdisziplin des Freifallformationsspringens.

Tabelle 11: Mittelwerte und Standardabweichungen der Erfahrungsgruppen im Freifallformationsspringen:

Fragebogenwerte und physiologische Aktivierung.

\begin{tabular}{|c|c|c|c|c|c|}
\hline & $\begin{array}{c}\text { Kognitive } \\
\text { Angst }\end{array}$ & $\begin{array}{c}\text { Somatische } \\
\text { Angst }\end{array}$ & $\begin{array}{c}\text { Physiologische } \\
\text { Aktivierung }\end{array}$ & $\begin{array}{c}\text { Selbstwirksamkeits- } \\
\text { überzeugung }\end{array}$ & Leistung \\
\hline $\begin{array}{c}\text { Niedrige } \\
\text { Erfahrung }\end{array}$ & $0,36(118)$ & $0,06(96)$ & $0,60(09)$ & $-0,07(99)$ & $-0,29(106)$ \\
\hline $\begin{array}{c}\text { Mittlere } \\
\text { Erfahrung }\end{array}$ & $-0,07(75)$ & $-0,03(107)$ & $0,58(09)$ & $-0,12(112)$ & $0,06(104)$ \\
\hline $\begin{array}{c}\text { Hohe } \\
\text { Erfahrung }\end{array}$ & $-0,25(98)$ & $-0,02(98)$ & $0,57(11)$ & $0,18(86)$ & $0,21(84)$ \\
\hline
\end{tabular}

${ }^{131}$ Die Zeitdauer von 5 Jahren im Fallschirmsport erscheint zunächst recht hoch: Zu beachten ist aber, dass nur zum Erlernen der Grundfertigkeiten und zum Erwerb der Fallschirmspringerlizenz häufig ein Jahr vergehen kann. Erst nach diesem Jahr kann dann eine sinnvolle Spezialausbildung im Freifallformationsspringen erfolgen. Zusätzlich reduziert sich aufgrund der klimatischen Gegebenheiten in Deutschland der für das Fallschirmspringen nutzbare Teil eines Jahres auf maximal 8 Monate. 
Die Werte aus Tabelle 11 sind in Abbildung 32 graphisch dargestellt. Man erkennt, dass für zunehmende Erfahrung die Angstkomponenten anscheinend abnehmen, wohingegen die subjektiv bewertete Leistung im Sprung zunimmt. Die statistische Relevanz dieser Beobachtung wird allerdings ebenfalls gesondert im Abschnitt 5.2.2 zur Hypothesenprüfung untersucht.

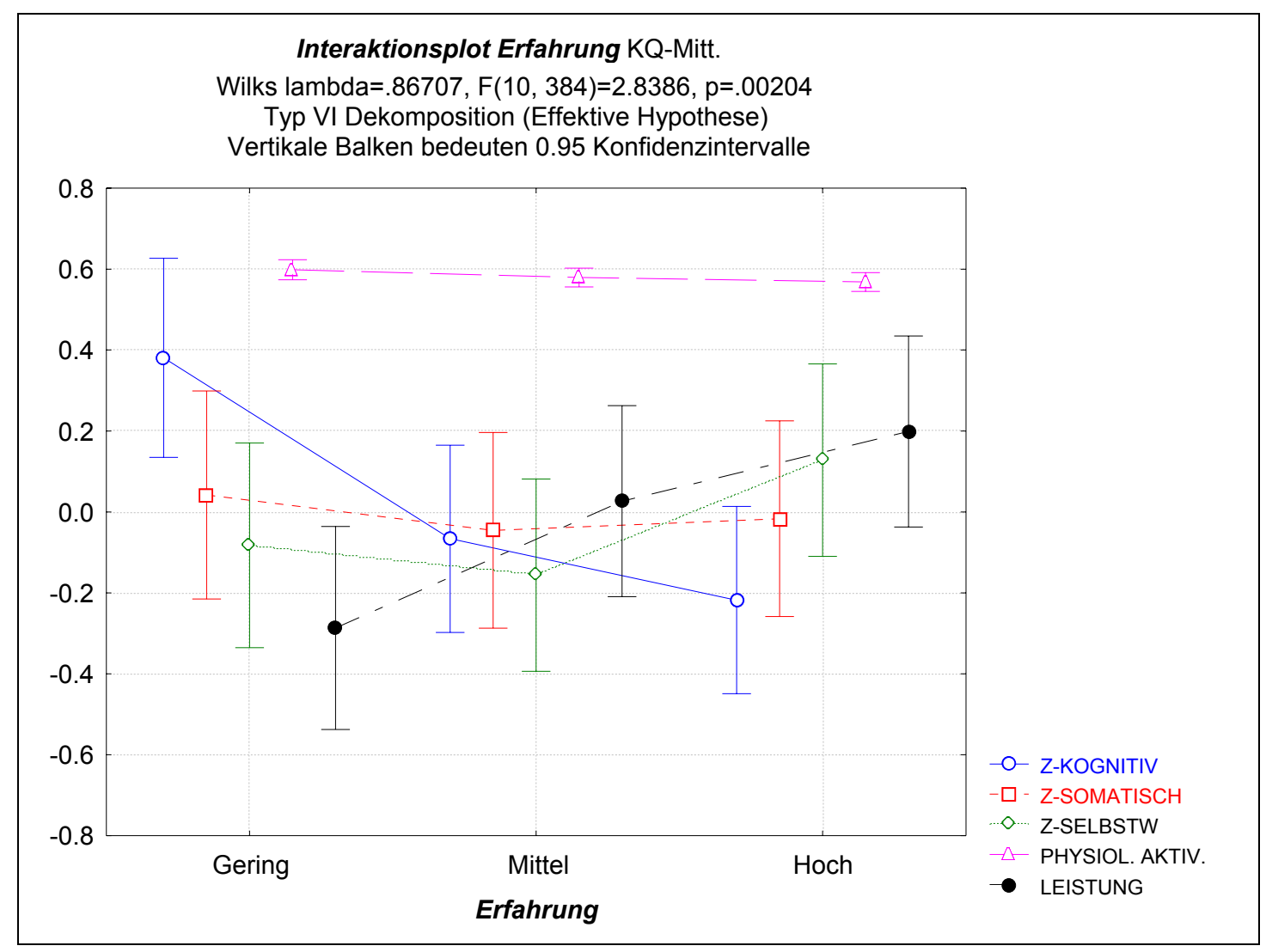

Abbildung 32: Interaktionsplot der Erfahrungsgruppen im Freifallformationsspringen:

Fragebogenwerte und physiologische Aktivierung.

\subsubsection{Korrelationen zwischen den Messgrößen}

Im Zuge einer weiteren statistischen Auswertung wurden die Korrelationskoeffizienten $r$ zwischen den Messgrößen berechnet. Ein erstes Augenmerk gilt hier einer Korrelation von der somatischen Angstkomponente des CSAI-2 mit der aus der Herzfrequenz der Probanden errechneten physiologischen Aktivierung. Da beide Größen ein Maß für die physiologische Erregung darstellen, wird eine Korrelation vermutet (vgl. PARFITT et al. 2000, 47). 
Leider lässt sich jedoch nur eine sehr schwache Korrelation ${ }^{132}(r=0,07)$ innerhalb des 95\%Konfidenzintervalls nachweisen. Dies könnte an der zeitlichen Verschiebung zwischen dem Moment der Angstreaktion und dem Ausfüllen des Fragebogens liegen (vgl. Abschnitt 2.2.4).

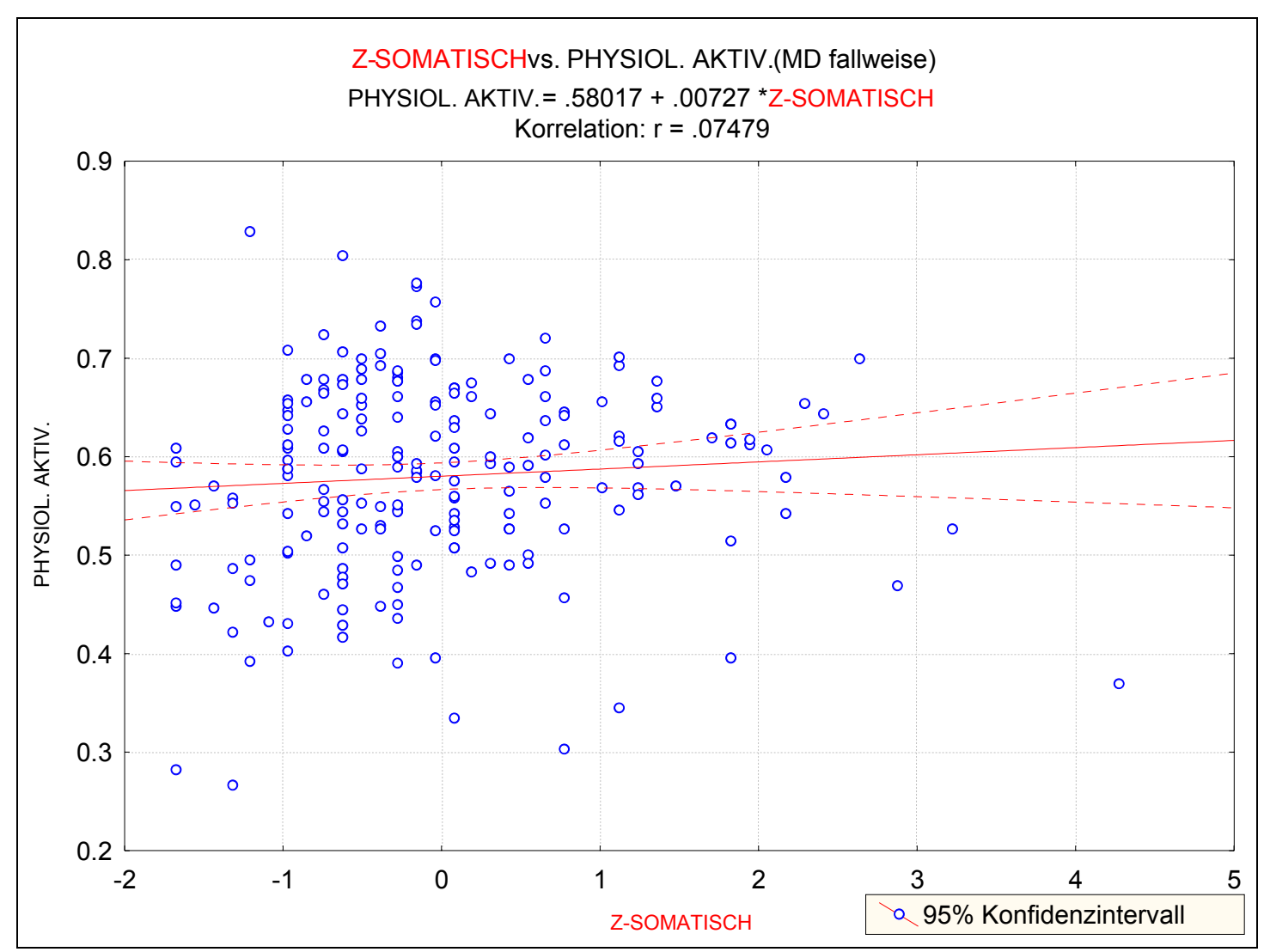

Abbildung 33: Korrelation von somatischer Angstkomponente und physiologischer Aktivierung.

Es mag aber auch sein, dass die Probanden im Freifall z.B. aufgrund der Kürze der Zeit oder der Ablenkung durch die Bewegungsaufgabe nicht in der Lage waren, ihren Körperzustand richtig wahrzunehmen. Für dieses Argument spricht u.a. die Tatsache, dass Frage 17 des CSAI-2 („Mein Herz rast") von den Probanden im Mittel während des Freifalls als „Ein wenig zutreffend “'133 beschrieben wird - obwohl die durchschnittliche Herzfrequenz aller Probanden im Freifall bei etwa 160 Schlägen pro Minute liegt ${ }^{134}$ !

\footnotetext{
${ }^{132}$ Werte des Korrelationskoeffizienten $r$ kleiner als 0,4 beschreiben nur einen sehr geringen Zusammenhang der beiden Größen. Dies wird anschaulich, wenn man wiederum das Gütemaß $r^{2}$ bildet: Aus dem $r$ von 0,4 wird $r^{2}=0,16$, es werden also nur 16\% der Varianz durch das Modell erklärt (vgl. WiLLIMCZIK 1997, 75f).

${ }^{133}$ In dem Bogen werden Gefühle hinsichtlich ihres momentanen Zutreffens in einer Skala von „Überhaupt nicht“ (1 Punkt), „Ein wenig“ (2 Punkte), „Ziemlich“ (3 Punkte) und „Sehr“ (4 Punkte) eingeordnet. Der Mittelwert der Probanden bei Frage 17 im Freifall liegt bei 1,79(80) Punkten.

${ }^{134}$ Siehe hierzu Abschnitt 5.1.1 auf Seite 108.
} 
Um dieser Vermutung weiter nachzugehen, wird im Folgenden die zeitliche Entwicklung der gemessenen Angstkomponenten während den beobachteten Fallschirmsprüngen betrachtet. Abbildung 34 zeigt einen Interaktionsplot dieser Messgrößen - hierbei wurde der Betrag der physiologischen Aktivierung für die Abbildung normiert.

Man erkennt die bereits in Abschnitt 5.1.1 beschriebene stetige Zunahme der physiologischen Aktivierung in der Phase vom Start des Flugzeuges bis zum Absprung mit anschließender Freifallphase.

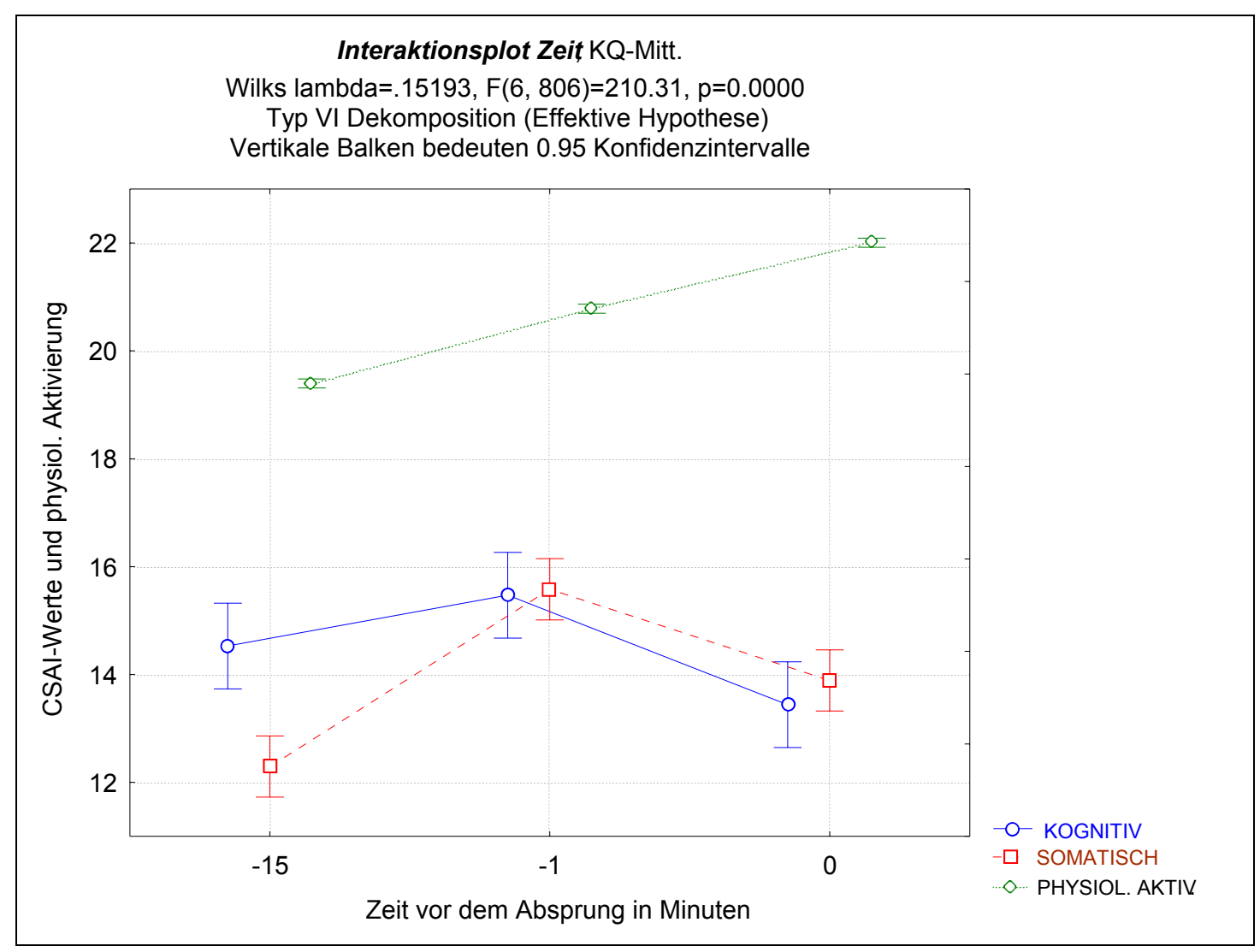

Abbildung 34: Mittelwerte der Angstkomponenten in Abhängigkeit des zeitlichen Sprungverlaufes.

Der Verlauf der somatischen Angstkomponente, die aus den CSAI-Fragebogenwerten ermittelt wurde, spiegelt diesen zeitlichen Verlauf jedoch nicht wider:

Die somatische Erregung nimmt zwar im Zeitraum von 15 Minuten $\left(M_{S O M, 15}=12,4\right)$ bis 1 Minute vor dem Absprung hoch signifikant ${ }^{135} \mathrm{zu}\left(M_{S O M, 1}=15,7 ; p=0,000\right)^{136}$, während der Freifallphase geben die Probanden allerdings eine hoch signifikant niedrigere somatische Angstkomponente an als eine Minute vor dem Absprung $\left(M_{S O M, 0}=14,0 ; p=0,000\right)$.

\footnotetext{
${ }^{135}$ Vgl. Anmerkung 146 auf Seite 133.

${ }^{136}$ Die statistische Signifikanz dieser Ausführungen wurde mit einer Varianzanalyse mit anschließendem SCHEFFÉ-Test überprüft.
} 
Es wäre somit zu vermuten, dass die untersuchten Fallschirmspringer tatsächlich nicht in der Lage waren, ihre Angstwerte und Körperreaktionen während der Freifallphase korrekt einzuschätzen. Die Mehrzahl der Probanden zeigte sich im nachhinein sehr erstaunt, dass sie während eines Sprunges die beschriebenen hohen Herzfrequenzwerte aufwiesen, wohingegen sie von der Selbstwahrnehmung her niedrigere Werte erwartet hätten. Für die Zukunft stellt sich dann die Frage, ob in der Ausbildung von Fallschirmspringern künftig die Selbstwahrnehmung des eigenen Körpers verstärkt geschult werden sollte.

Andererseits könnte die deutliche Absenkung der kognitiven und somatischen Angstkomponente während der Freifallphase auch durch bereits erfolgende Angstbewältigung begründet werden.

Um aus diesem abweichenden zeitlichen Verlauf für die weitere Auswertung der Untersuchung bei der Verwendung der Fragebogenwerte keine systematischen Fehler zu erhalten, werden im Folgenden stets die Mittelwerte der Angstkomponenten, wie bereits in Abschnitt 3.2 zur Untersuchungsmethodik beschrieben, verwendet. Zusätzlich kommt der physiologischen Aktivierung als objektive Messgröße eine steigende Bedeutung zu.

Weiterhin wurden die Korrelationen zwischen Angstkomponenten und der im Sprung erbrachten Leistung betrachtet:

So wird im Rahmen der multidimensionalen Angsttheorie von MARTENS ein negativ linearer Zusammenhang von kognitiver Angstkomponente und Leistung, ein positiv linearer Einfluss der Selbstwirksamkeitsüberzeugung und ein umgekehrt U-förmiger Zusammenhang von somatischer Angstkomponente und Leistung vorhergesagt.

Tabelle 12: Korrelationskoeffizienten r von Angstkomponenten mit der Leistung in den Ängstlichkeitsgruppen

\begin{tabular}{|c|c|c|c|c|}
\hline & $\begin{array}{c}\text { Kognitive } \\
\text { Angst }\end{array}$ & $\begin{array}{l}\text { Selbstwirksamkeits- } \\
\text { überzeugung }\end{array}$ & $\begin{array}{c}\text { (Somatische } \\
\text { Angst) }\end{array}$ & \begin{tabular}{|c} 
(Physiologische \\
Aktivierung) $^{2}$
\end{tabular} \\
\hline Gesamt & $-0,17$ & 0,21 & $-0,07$ & $-0,03$ \\
\hline $\begin{array}{c}\text { Gruppe 1: } \\
\text { Niedrige } \\
\text { Ängstlichkeit }\end{array}$ & $-0,22$ & 0,19 & $-0,03$ & $-0,06$ \\
\hline $\begin{array}{c}\text { Gruppe 2: } \\
\text { Mittlere } \\
\text { Ängstlichkeit }\end{array}$ & $-0,18$ & 0,22 & $-0,10$ & 0,02 \\
\hline $\begin{array}{c}\text { Gruppe 3: } \\
\text { Hohe } \\
\text { Ängstlichkeit }\end{array}$ & $-0,26$ & 0,26 & $-0,13$ & $-0,10$ \\
\hline
\end{tabular}


Die jeweiligen Korrelationen sind allerdings erneut nur sehr gering: Die kognitive Angstkomponente korreliert schwach negativ $(r=-0,17)$ mit der Leistung, die Selbstwirksamkeitsüberzeugung schwach positiv $(r=0,21)$, die Quadrate von somatischer Angstkomponente $(r=-0,07)$ und physiologischer Aktivierung $(r=-0,03)$ zeigen keinen statistisch signifikanten Zusammenhang mit der im Sprung erbrachten Leistung.

Betrachtet man die Korrelationen der Fragebogenwerte untereinander, fällt auf, dass auch innerhalb dieser Werte schwache Korrelationen zu erkennen sind. So korreliert die kognitive Angstkomponente negativ mit der Selbstwirksamkeit $(r=-0,55)$. Dies ist zu erwarten, denn diese beiden Komponenten des Fragebogens können nach MARTENS et al. (1990) als zwei Gegenpole einer kognitiven Skala angesehen werden (vgl. Abschnitt 1.1.4 auf Seite 8). In neueren Untersuchungen haben sich allerdings die kognitive Komponente der Zustandsangst und die Selbstwirksamkeit als relativ unabhängig voneinander erwiesen - zumindest, wenn man betrachtet, in welchem Maße diese Größen auf den Angst-Leistungs-Zusammenhang einwirken (vgl. EDWARDS et al. 2002, 11).

Weiterhin zeigen auch die somatische Angstkomponente $(r=0,50)$ und die physiologische Aktivierung $(r=0,16)$ eine geringe positive Korrelation mit der kognitiven Zustandsangst.

\subsection{Statistische Ergebnisse}

Zum indirekten Testen des Katastrophenmodells von Angst und sportlicher Leistung beim Freifallformationsspringen und zur Hypothesenprüfung wurde der t-Test für unabhängige Stichproben angewendet.

Für die Prüfung der Mittelwertunterschiede von mehr als zwei Gruppen wurde eine einfaktorielle Varianzanalyse (ANOVA) mit nachfolgendem SCHEFFÉ-Test zur Ermittlung, welche Gruppendifferenzen signifikant sind, durchgeführt.

Der Nachweis von signifikant geringeren quadrierten Abweichungssummen ${ }^{137} \phi$ beim Testen, ob die Katastrophenmodelle den Angst-Leistungs-Zusammenhang enger beschreiben als konventionelle Modelle, wurde zusätzlich mit dem WILCOXON-Test für gepaarte Stichproben geführt.

\footnotetext{
${ }^{137}$ Vgl. hierzu Abschnitt 4.4.3.2 auf Seite 71 zum Vergleich konkurrierender Katastrophenmodelle.
} 


\subsection{1 Überprüfung des Katastrophenmodells}

Nach ZEEMAN (1995, 5) und HARDy (1996b, 144f) sollte jeder Anpassungsversuch eines Katastrophenmodells zunächst mit dem Nachweis der Nicht-Eignung konventioneller Modelle erfolgen. Hierzu wurde versucht, die Messwerte mit dem Programm DATAFIT an ein multidimensionales Angstmodell, realisiert durch Gleichung 7, anzupassen.

Gleichung 7: Modellierung des multidimensionalen Angstmodells (erweitert nach EDWARDS/HARDY 1996, 303).

$$
\text { LEISTUNG }=a+b(\text { KOG })+c(S O M)+d(\text { PHYSIO })+e(S O M)^{2}+f(P H Y S I O)^{2}+g(S W)
$$

Diese mathematische Modellierung beschreibt eine gebogene Leistungsoberfläche (siehe Abbildung 5 auf Seite 31) und verwendet sowohl die somatische Angstkomponente als auch die physiologische Aktivierung. KOG und SOM stellen die kognitive und somatische Angstkomponente, PHYSIO die physiologische Aktivierung und SW die Selbstwirksamkeitsüberzeugung dar. Die Koeffizienten $a$ bis $g$ werden zur Anpassung der Messwerte an die Leistungsoberfläche genutzt.

Der Vorteil dieser Formel ist, dass sie alle simpleren Angst-Leistungs-Modelle enthält: So kann durch Nullsetzen der Koeffizienten $b, c$ und $e$ ein reiner umgekehrt U-förmiger Zusammenhang dargestellt werden - Gleichung 7 ist also geeignet, alle Angst-LeistungsModelle aus Abschnitt 3.1 bis zur Komplexität der multidimensionalen Angsttheorie von MARTENS zu testen.

Insgesamt konnten mit diesem Modell jedoch nur 6,82 Prozent der Leistungsvarianz $\left(r^{2}=0,07\right)$ erklärt werden; eine Unterscheidung der Ängstlichkeitsgruppen bringt nur eine geringe Verbesserung der Gütemaße: In Gruppe 1 der niedrigen Eigenschaftsangst werden 13,95 Prozent $\left(r^{2}=0,14\right)$, in Gruppe 2 der mittleren Ängstlichkeit 7,61 Prozent $\left(r^{2}=0,08\right)$ und in Gruppe 3 der hohen Eigenschaftsangst 16,45 Prozent $\left(r^{2}=0,16\right)$ der Leistungsvarianz durch die Angst- und Aktivierungskomponenten erklärt.

\subsubsection{Untersuchung auf chaotisches Verhalten mit dem Ljapunov-Exponenten}

Wie in Abschnitt 4.4.1 auf Seite 66 erläutert, lässt sich aus den erhobenen Untersuchungsdaten der Ljapunov-Exponent $\lambda$ berechnen. Sollte der betrachtete Angst-Leistungs-Zusammenhang durch ein Katastrophenmodell beschreibbar sein, so existiert mindestens ein 
stationärer Systemzustand: Der Ljapunov-Exponent muss demnach einen negativen Wert haben. Andernfalls verhält sich das untersuchte System rein chaotisch.

Die Berechnung des Ljapunov-Exponenten $\lambda$ mit den Programmen DATAFIT und EXCEL nach der von GuAstello (2002) vorgeschlagenen Methode ${ }^{138}$ liefert einen Wert des angepassten Exponenten von $\lambda=-0,522(316)$.

Somit existiert mindestens ein stationärer Systemzustand: Der betrachtete Angst-LeistungsZusammenhang ist nicht chaotisch und nicht exakt durch ein lineares oder multidimensionales Angstmodell darstellbar. Daher erfolgt die weitere Auswertung im Rahmen der Katastrophentheorie.

\subsubsection{Indirektes Testen auf Katastropheneigenschaften}

Abschnitt 4.4.2 des theoretischen Teils ${ }^{139}$ beschrieb bereits, wie zum indirekten Testen eines Katastrophenmodells versucht werden kann, Katastropheneffekte nachzuweisen. Anhand Tabelle 5 (vgl. S. 68) lassen sich folgende Hypothesen aufstellen:

H9: Ist die kognitive Angstkomponente klein, so existiert bei niedriger und hoher physiologischer Aktivierung kein Leistungsunterschied.

H10: Ist die kognitive Angstkomponente groß, so ist die Leistung bei hoher physiologischer Aktivierung signifikant geringer als bei niedriger Aktivierung.

Zur Einordnung der niedrigen und hohen Zustandsangst- bzw. Aktivierungssituation wurde erneut eine Messgröße als niedrig eingeordnet, wenn die z-Werte kleiner als -0,5 waren; für z-Werte größer als 0,5 wurde sie als hoch definiert.

Aufgrund einer zu geringen Datenmenge war es nicht möglich, die t-Tests zur Prüfung der Zusatzhypothesen in den drei Ängstlichkeitsgruppen durchzuführen, daher erfolgt eine Betrachtung aller Daten:

Innerhalb der Gesamtgruppe existieren bei kleiner kognitiver Angstkomponente keine signifikanten Leistungsunterschiede zwischen hoher und niedriger physiologischer Aktivierung $(p=0,64)$ oder somatischer Angstkomponente $(p=0,95)$.

Damit kann H9 zumindest für die Gesamtuntersuchungsgruppe bestätigt werden.

\footnotetext{
${ }^{138}$ Siehe Gleichung 2 auf S. 67.

${ }^{139}$ Siehe S. 67.
} 
Leider aber zeigt auch ein t-Test von hoher und niedriger physiologischer Aktivierung bei großer kognitiver Angstkomponente keine signifikanten Leistungsunterschiede $(p=0,51)$. Betrachtet man stattdessen die somatische Angstkomponente, existiert zwar ein signifikanter Leistungsunterschied $(p=0,04)$, jedoch sind die Unterschiede nicht mit den Vorhersagen des Katastrophenmodells verträglich: Niedrige somatische Angst ist mit schlechter, hohe somatische Angst mit unterdurchschnittlicher Leistung verknüpft.

Damit muss H10 abgelehnt werden, ein indirekter statistischer Nachweis eines Katastrophenmodells ist aus den Messdaten nicht möglich. Ob dieser Nachweis geglückt wäre, wenn aufgrund eines größeren Datenbestands eine gesonderte Betrachtung der Ängstlichkeitsgruppen möglich gewesen wäre, bleibt offen.

\subsubsection{Anpassung von Katastrophenflächen mit dem Programm GEMCAT II}

Wie bereits in Abschnitt 4.4.3.2 erläutert, ist es mit dem Programm GEMCAT II möglich, Katastrophenmodelle quantitativ anzupassen. Dazu schätzt das Programm die Koeffizienten $\chi, \beta, \alpha$ für jede der genutzten Variablen (siehe Abschnitt 4.4.3.2 auf Seite 71) und gibt ein pseudo- $r^{2}$ als Gütemaß der Gesamtanpassung des genutzten Katastrophenmodells aus.

Da das Programm GEMCAT II es ermöglicht, eine Kontrollvariable von mehreren Messgrößen beeinflussen zu lassen, wurden zur Anpassung eines Spitzenkatastrophenmodells von Angst und sportlicher Leistung für den Normalfaktor $a$ die physiologische Erregung und die somatische Angstkomponente gewählt. Der spaltende Faktor $b$ wurde aus kognitiver und somatischer Angstkomponente sowie aus der Selbstwirksamkeitsüberzeugung gebildet. Auf diese Art und Weise konnten die beobachteten Korrelationen der Fragebogenwerte untereinander optimal im Katastrophenmodell berücksichtigt werden.

Aus der Grundgleichung ${ }^{140} x^{3}+b x+a=0$ der Spitzenkatastrophe erhält man dann für die Optimierungsgleichung, deren Koeffizienten vom Programm geschätzt wurden, die in Gleichung 8 auf der folgenden Seite dargestellte Form.

\footnotetext{
${ }^{140} \mathrm{Vgl}$. Anm. 70 auf Seite 71.
} 
Gleichung 8: Optimierungsgleichung des Spitzenkatastrophenmodells von Angst und sportlicher Leistung bei Fallschirmspringern (vgl. LANGE et al. 2001, 138f).

$$
\begin{aligned}
& {\left[\left(0,5 \cdot(\text { LEISTUNG }+\chi)^{3}\right.\right.} \\
& +\left(\beta_{1} \cdot \text { KOG }+\beta_{2} \cdot S O M+\beta_{3} \cdot S W+\beta_{4}\right) \cdot(0,5 \cdot(\text { LEISTUNG }+\chi) \\
& \left.+\left(\alpha_{1} \cdot S O M+\alpha_{2} \cdot \text { PHYSIO }+\alpha_{3}\right)\right]=0
\end{aligned}
$$

Tabelle 13 zeigt die Koeffizienten der vom Programm GEMCAT II angepassten Katastrophenoberflächen in den drei Gruppen unterschiedlicher Eigenschaftsangst.

Die Gütemaße für die Anpassungen dieser Katastrophenflächen an die Messwerte der drei Ängstlichkeitsgruppen liegen durchweg bei hohen Werten, in allen Gruppen werden mindestens 63\% der Leistungsvarianz durch das Katastrophenmodell erklärt.

Tabelle 13: Koeffizienten und Fehler der angepassten Spitzenkatastrophenmodelle in den Ängstlichkeitsgruppen.

\begin{tabular}{|c|c|c|c|}
\hline & $\begin{array}{c}\text { Gruppe 1: Niedrige } \\
\text { Ängstlichkeit }\end{array}$ & $\begin{array}{c}\text { Gruppe 2: Mittlere } \\
\text { Ängstlichkeit }\end{array}$ & $\begin{array}{c}\text { Gruppe 3: Hohe } \\
\text { Ängstlichkeit }\end{array}$ \\
\hline Pseudo- ${ }^{2}$ & $\mathbf{0 , 6 3 0}$ & $\mathbf{0 , 8 7 8}$ & $\mathbf{0 , 8 2 1}$ \\
\hline$\alpha_{1}$ (SOM) & $0,095(19)$ & $0,029(8)$ & $0,058(27)$ \\
\hline$\alpha_{2}$ (PHYSIO) & $0,477(26)$ & $-0,424(36)$ & $-0,146(34)$ \\
\hline$\alpha_{3}$ (Konstante) & $-0,724(33)$ & $0,135(18)$ & $-0,016(6)$ \\
\hline$\beta_{1}$ (KOG) & $-0,173(15)$ & $0,092(24)$ & $0,029(34)$ \\
\hline$\beta_{2}$ (SOM) & $0,012(14)$ & $-0,216(47)$ & $-0,258(105)$ \\
\hline$\beta_{3}$ (SW) & $0,041(14)$ & $0,182(30)$ & $0,021(5)$ \\
\hline$\beta_{4}$ (Konstante) & $1,525(146)$ & $0,711(31)$ & $0,762(136)$ \\
\hline$\chi$ (Konstante) & $0,661(72)$ & $0,357(45)$ & $0,337(111)$ \\
\hline
\end{tabular}

Diese angepassten Katastrophenflächen sind in Abbildung 35 auf der folgenden Seite graphisch dargestellt. Für alle Ängstlichkeitsgruppen erkennt man die typische Form der brechenden Welle. Dabei verschiebt sich mit zunehmender Ängstlichkeit die Position, an der sich die Katastrophenfläche faltet.

Weiterhin verändert sich der Grad der Überlappung von den zwei Ebenen hoher und niedriger Leistung: Während in der Gruppe 1 mit niedriger Ängstlichkeit geringe Werte der physiologischen Aktivierung eindeutig der oberen Leistungsebene zuzuordnen sind, können diese in Gruppe 3 der hohen Ängstlichkeit sowohl der oberen als auch der unteren Leistungsebene zugeordnet werden. 


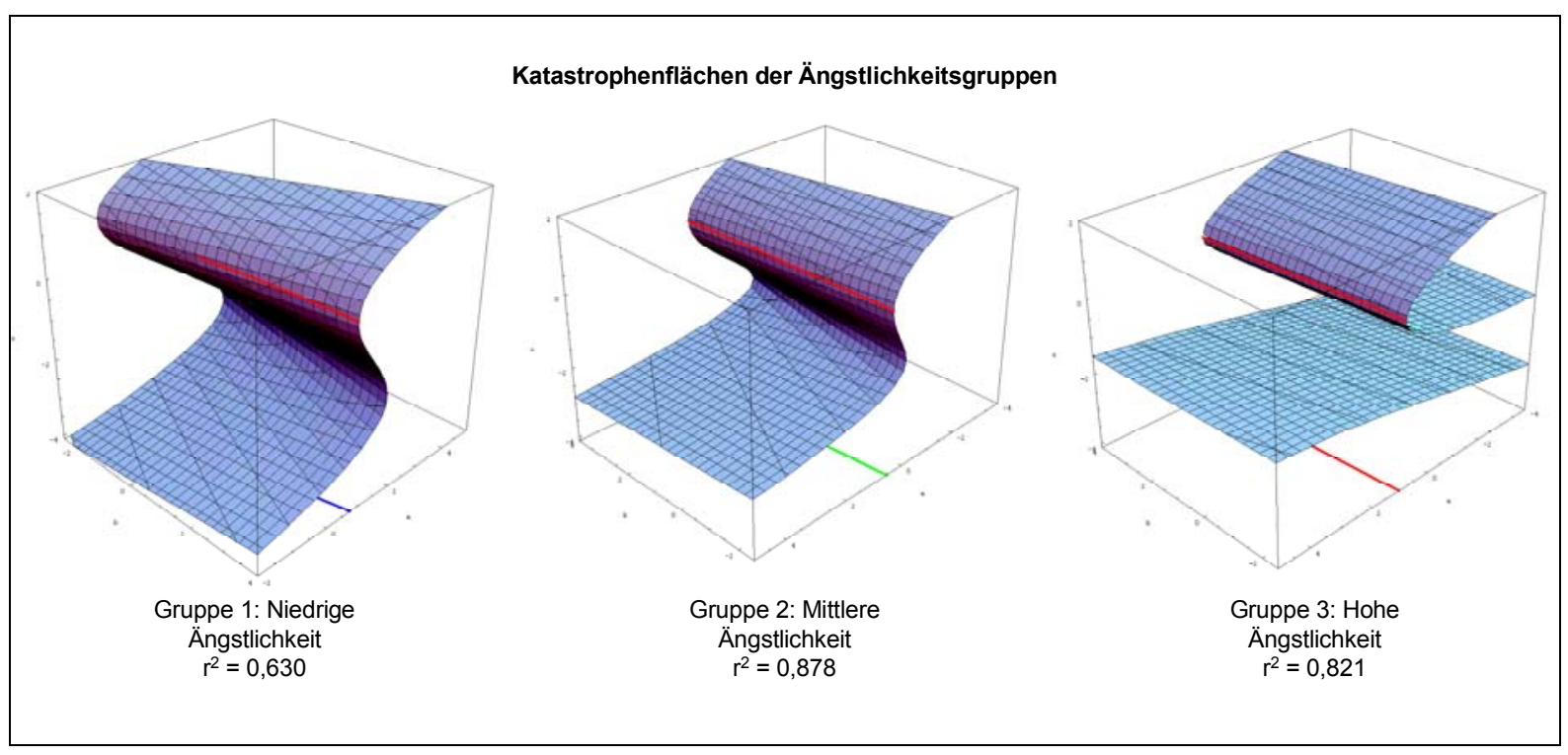

Abbildung 35: Katastrophenflächen für unterschiedliche Eigenschaftsangst.

Bei einer Deutung der Katastrophenflächen sollte der wirklich durchlaufene Wertebereich der Messdaten berücksichtigt werden. Abbildung 36 zeigt die Kontrollebene der Katastrophenflächen für die drei Ängstlichkeitsgruppen. Die Projektionen der Kanten der brechenden Wellen für die drei Oberflächen sind in dieser Abbildung zu sehen (blau: Gruppe 1; grün: Gruppe 2; rot: Gruppe 3) - der Bereich, in dem alle Messwerte liegen, ist grau eingefärbt.

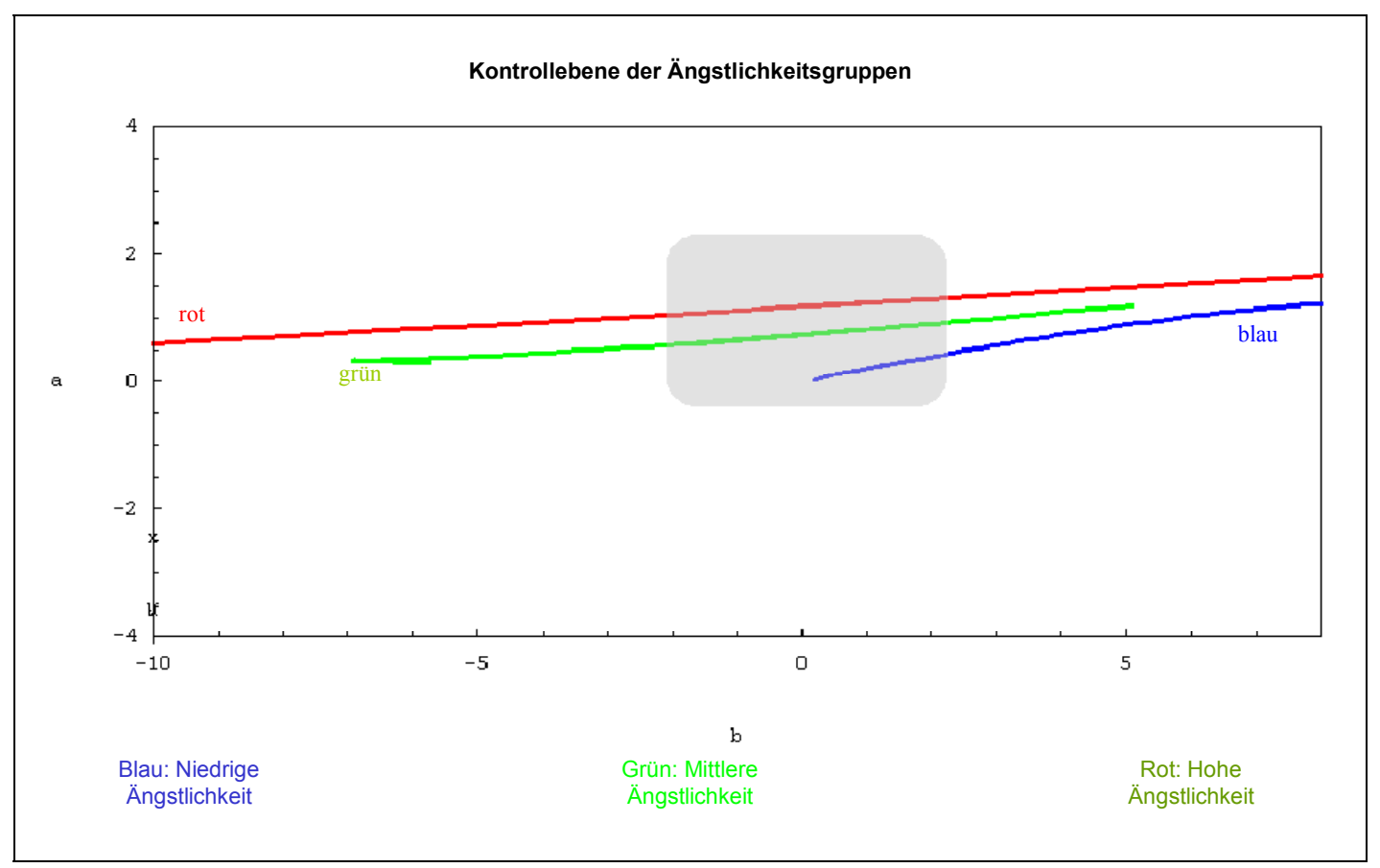

Abbildung 36: Kontrollebene für Gruppen unterschiedlicher Eigenschaftsangst. 
Aus den Projektionen der Wellenkanten wird ersichtlich, dass für alle Ängstlichkeitsgruppen grundsätzlich mit dem Auftreten von Katastropheneffekten zu rechnen ist. In allen Gruppen ist ein Übergang von der oberen zur unteren Leistungsebene nur durch Katastrophensprünge möglich, ein stetiger Leistungsübergang scheint nicht möglich.

Man erkennt, dass die Messwerte für Gruppe 1 (niedrige Eigenschaftsangst) dabei nur in einem kleinen Bereich in der Region der blauen Kurve liegen, während sich der Großteil der Messwerte in der Region der oberen Leistungsoberfläche befindet. Katastropheneffekte können nur in dem kleinen Bereich auftreten, der sich in Abbildung 36 unterhalb der blauen Kurve befindet.

Die Messwerte von Gruppe 2 liegen etwa zur Hälfte im Bereich der oberen Leistungsebene ${ }^{141}$, wohingegen in Gruppe 3 bereits die Mehrzahl der Messwerte unterhalb der roten Projektionskurve liegt und somit mit schlechteren Leistungen verknüpft ist. Dieser Effekt wird noch durch die starke Faltung der Katastrophenfläche bei der Gruppe hoher Ängstlichkeit verstärkt: Auch einige der Messwerte oberhalb der roten Projektionskurve können bereits mit niedrigen Leistungswerten verknüpft sein.

Es fällt auf, dass die Katastrophenoberflächen der drei Ängstlichkeitsgruppen ausgesprochen ähnlich aussehen: Die Projektionskurven der Wellenkanten verlaufen nahezu parallel. Somit könnte der Eigenschaftsangst bei einem Katastrophenmodell von Angst und sportlicher Leistung die Rolle eines verschiebenden Faktors in Richtung des Normalfaktors $a$ zukommen.

Da der Selbstwirksamkeitsüberzeugung und der Sprungerfahrung zusätzliche steuernde Rollen innerhalb des Angst-Leistungs-Zusammenhangs zugeschrieben werden, erfolgen im Rahmen der weiteren Auswertung zwei Versuche, diesen Messgrößen hinreichendes Gewicht zu verleihen. Zunächst wurde mit dem Programm GEMCAT II eine Schmetterlingskatastrophenfläche an die Messwerte angepasst. Dabei wurden wieder physiologische Erregung und somatische Angstkomponente als Normalfaktor $a$, kognitive und somatische Angstkomponente sowie die Selbstwirksamkeit als spaltenden Faktor $b$ und die Selbstwirksamkeit (wie von HARDY vorgeschlagen) als Verschiebungsfaktor $c$ eingesetzt. Für den

\footnotetext{
${ }^{141}$ Der Bereich der oberen Leistungsebene für Gruppe 1 und 2 reicht in Abbildung 36 jeweils vom oberen Rand der Grafik herab bis zur Projektion der Wellenkante. Aufgrund der starken Faltung der Katastrophenfläche von der Gruppe 3 (hohe Ängstlichkeit) ist allerdings zu beachten, dass für diese Gruppe auch oberhalb der roten Projektionskurve die niedrige Leistungsebene erreicht werden kann.
} 
Schmetterlingsfaktor $d$ wurden spekulativ die Anzahl von Formationssprüngen und die springerische Erfahrung (in Jahren) der Probanden verwendet.

Gleichung 9: Optimierungsgleichung des Schmetterlingskatastrophenmodells von Angst und sportlicher Leistung bei Fallschirmspringern (vgl. LANGE et al. 2001, 138f).

$$
\begin{aligned}
& {\left[\left(0,5 \cdot(\text { LEISTUNG }+\chi)^{5}\right.\right.} \\
& +\left(\alpha_{1} \cdot S O M+\alpha_{2} \cdot \text { PHYSIO }+\alpha_{3}\right) \\
& +\left(\beta_{1} \cdot \text { KOG }+\beta_{2} \cdot S O M+\beta_{3} \cdot S W+\beta_{4}\right) \cdot(0,5 \cdot(\text { LEISTUNG }+\chi) \\
& +\left(\gamma_{1} \cdot S W+\gamma_{2}\right) \cdot\left(0,5 \cdot(\text { LEISTUNG }+\chi)^{2}\right. \\
& +\left(\delta_{1} \cdot J A H R E+\delta_{2} \cdot S P R \ddot{U N G E}+\delta_{3}\right) \cdot\left(0,5 \cdot(\text { LEISTUNG }+\chi)^{3}\right]=0
\end{aligned}
$$

Die Anpassung der Messwerte aus den drei Ängstlichkeitsgruppen mittels Gleichung 9 lieferte nahezu perfekte Gütemaße im Bereich von über 98\% erklärter Leistungsvarianz. Die erhaltenen Werte und Fehler für die Koeffizienten sind in Tabelle 14 dargestellt.

Tabelle 14: Koeffizienten und Fehler der angepassten Schmetterlingskatastrophenmodelle in den Ängstlichkeitsgruppen.

\begin{tabular}{|c|c|c|c|}
\hline & $\begin{array}{c}\text { Gruppe 1: Niedrige } \\
\text { Ängstlichkeit }\end{array}$ & $\begin{array}{c}\text { Gruppe 2: Mittlere } \\
\text { Ängstlichkeit }\end{array}$ & $\begin{array}{c}\text { Gruppe 3: Hohe } \\
\text { Ängstlichkeit }\end{array}$ \\
\hline Pseudo-r $^{\mathbf{2}}$ & $\mathbf{0 , 9 8 4}$ & $\mathbf{0 , 9 8 3}$ & $\mathbf{0 , 9 8 9}$ \\
\hline$\alpha_{1}$ (SOM) & $-0,022(10)$ & $0,004(3)$ & $0,015(10)$ \\
\hline$\alpha_{2}$ (PHYSIO) & $-0,011(28)$ & $0,018(10)$ & $-0,047(27)$ \\
\hline$\alpha_{3}$ (Konstante) & $0,094(7)$ & $0,022(1)$ & $0,052(23)$ \\
\hline$\beta_{1}$ (KOG) & $0,044(13)$ & $0,020(14)$ & $0,031(11)$ \\
\hline$\beta_{2}$ (SOM) & $0,008(8)$ & $-0,051(24)$ & $-0,053(38)$ \\
\hline$\beta_{3}$ (SW) & $-0,003(25)$ & $0,070(83)$ & $-0,034(38)$ \\
\hline$\beta_{4}$ (Konstante) & $-0,521(159)$ & $-0,386(156)$ & $-0,271(95)$ \\
\hline$\gamma_{1}$ (SW) & $-0,022(56)$ & $-0,147(76)$ & $0,054(68)$ \\
\hline$\gamma_{2}$ (Konstante) & $-0,887(47)$ & $-0,099(67)$ & $-0,231(29)$ \\
\hline$\delta_{1}($ Jahre im Sport) & $-0,016(9)$ & $-0,016(22)$ & $0,014(7)$ \\
\hline$\delta_{2}$ (FS-Sprünge) & $0,000(0)$ & $0,000(0)$ & $0,000(0)$ \\
\hline$\delta_{3}$ (Konstante) & $2,553(338)$ & $1,458(366)$ & $1,414(297)$ \\
\hline$\chi$ (Konstante) & $0,515(93)$ & $0,305(131)$ & $0,304(95)$ \\
\hline
\end{tabular}


Aufgrund der Fünfdimensionalität der Schmetterlingskatastrophe ist leider eine graphische Darstellung und Deutung dieser Ergebnisse nicht möglich ${ }^{142}$. Als Folge wird davor gewarnt, das Schmetterlingsmodell nur aufgrund der Basis hoher Gütemaße zu akzeptieren; die Werte erscheinen fast als ,,zu gut“ - das Modell der Schmetterlingskatastrophe beschrieb bei HARDY (1996) nur ein Prozent mehr Leistungsvarianz (vgl. HARDY 1996b, 81), hier beträgt die Steigerung mindestens 12 Prozent.

Um die Selbstwirksamkeitsüberzeugung und die Sprungerfahrung in ein einfacheres Modell der Spitzenkatastrophe integrieren zu können, erfolgt zunächst eine Anpassung der Messdaten innerhalb der Gruppen unterschiedlich hoher Selbstwirksamkeit (vgl. Abschnitt 5.1.2).

Der Optimierungsansatz ist hier wiederum ein Spitzenkatastrophenmodell von Angst und sportlicher Leistung nach Gleichung 8.

Tabelle 15: Koeffizienten und Fehler der angepassten Spitzenkatastrophenmodelle in den Gruppen unterschiedlicher Selbstwirksamkeitsüberzeugung.

\begin{tabular}{|c|c|c|c|}
\hline & $\begin{array}{c}\text { Z-SELBSTW<-0,5: } \\
\text { Niedrige } \\
\text { Selbstwirksamkeits- } \\
\text { ubberzeugung }\end{array}$ & $\begin{array}{c}-\mathbf{0 , 5}<\text { Z-SELBSTW<0,5: } \\
\text { Mittlere } \\
\text { Selbstwirksamkeits- } \\
\text { überzeugung }\end{array}$ & $\begin{array}{c}\mathbf{0 , 5}<\text { Z-SELBSTW: } \\
\text { Hohe } \\
\text { Selbstwirksamkeits- } \\
\text { überzeugung }\end{array}$ \\
\hline${\text { Pseudo- }{ }^{2}}^{\mathbf{0}}$ & $\mathbf{0 , 8 5 0}$ & $\mathbf{0 , 6 6 6}$ & $\mathbf{0 , 6 1 3}$ \\
\hline$\alpha_{1}$ (SOM) & $0,017(1)$ & $0,338(130)$ & $0,344(112)$ \\
\hline$\alpha_{2}$ (PHYSIO) & $0,003(26)$ & $-0,201(91)$ & $-0,735(288)$ \\
\hline$\alpha_{3}$ (Konstante) & $-0,065(7)$ & $-0,128(9)$ & $0,253(146)$ \\
\hline$\beta_{1}$ (KOG) & $-0,009(22)$ & $-0,155(52)$ & $0,048(30)$ \\
\hline$\beta_{2}$ (SOM) & $-0,084(37)$ & $-0,528(203)$ & $-0,567(176)$ \\
\hline$\beta_{3}$ (SW) & $0,448(162)$ & $0,198(80)$ & $0,052(23)$ \\
\hline$\beta_{4}$ (Konstante) & $1,274(290)$ & $1,161(200)$ & $1,016(182)$ \\
\hline$\chi$ (Konstante) & $0,393(64)$ & $0,554(127)$ & $0,489(113)$ \\
\hline
\end{tabular}

In den beiden Gruppen mit mittlerer und hoher Selbstwirksamkeitsüberzeugung erkennt man wieder die typische Form der Katastrophenfläche; die in Abbildung 37 auf der folgenden Seite graphisch dargestellt wurde.

\footnotetext{
${ }^{142}$ Die dreidimensionale Darstellung der Schmetterlingskatastrophe ist nur möglich, wenn zwei der vier Kontrollvariablen konstant gesetzt werden. Da dieses Konstantsetzen aber das angepasste Modell verfälschen würde, wird auf die graphische Darstellung und Interpretation verzichtet.
} 


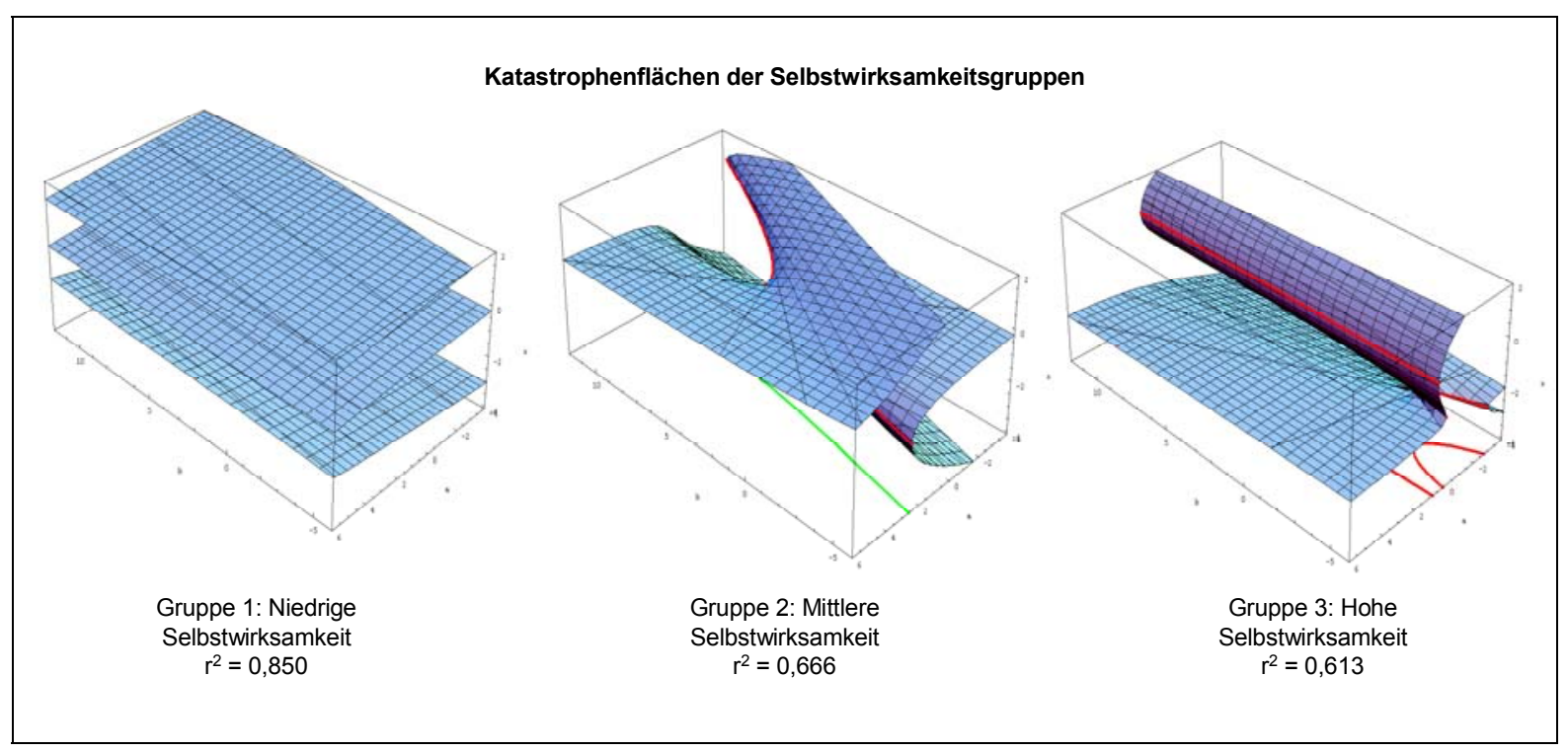

Abbildung 37: Katastrophenflächen für unterschiedliche Selbstwirksamkeitsüberzeugung.

$\mathrm{Zu}$ einer genaueren Deutung wird wieder die Projektion der „Wellenkanten“ auf die Kontrollebene mit herangezogen. Diese Projektion ist in Abbildung 38 dargestellt (blau: niedrige, grün: mittlere und rot: hohe Selbstwirksamkeitsüberzeugung) - der in den Untersuchungen durchlaufene Wertebereich ist wiederum grau eingefärbt ${ }^{143}$.

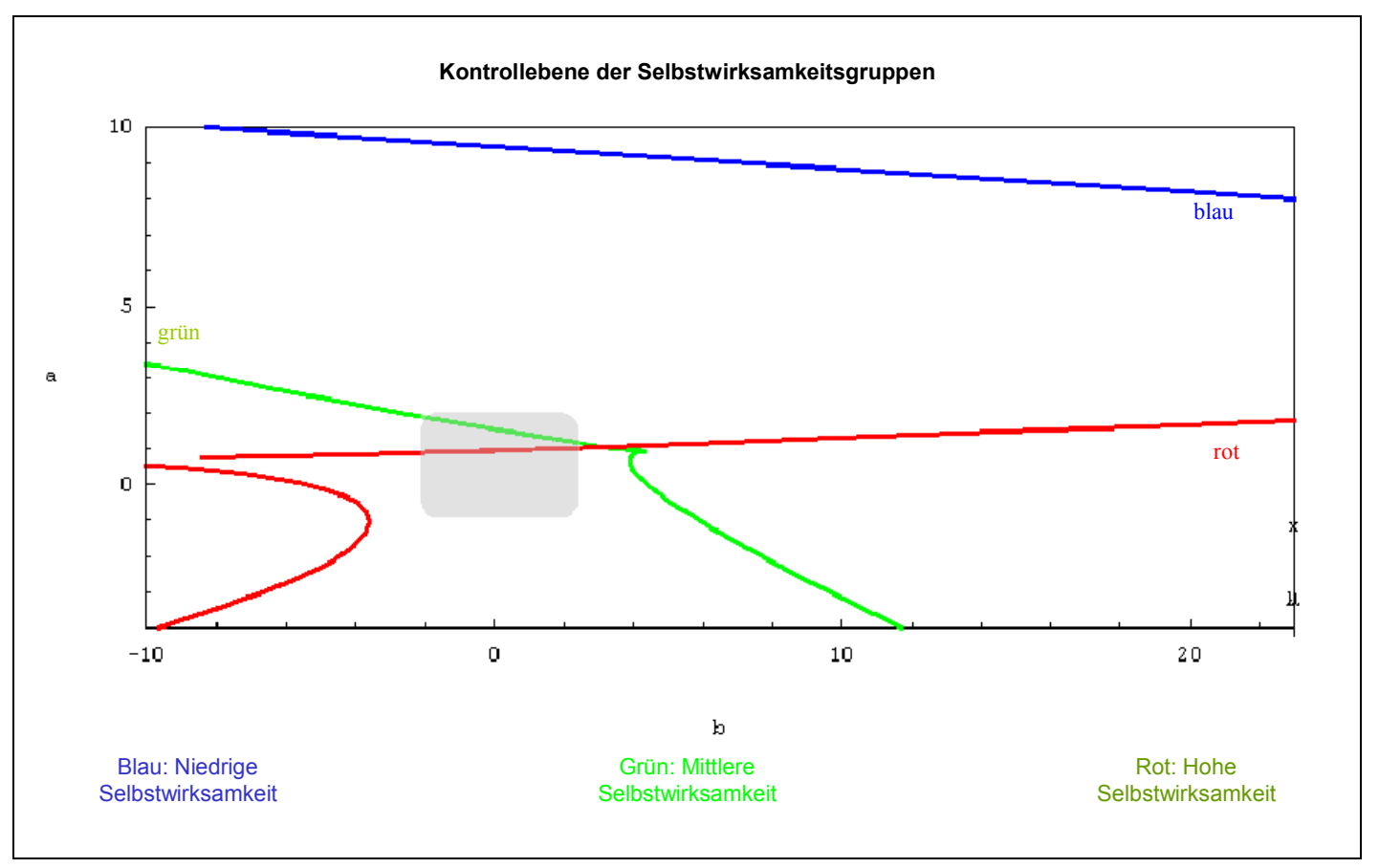

Abbildung 38: Kontrollebene für Gruppen unterschiedlicher Selbstwirksamkeitsüberzeugung.

${ }^{143} \mathrm{Zu}$ beachten ist, dass für Abbildung 37 und Abbildung 38 jeweils ein kleinerer Maßstab gewählt wurde, um die Katastrophenflächen und die blaue Projektionskurve vollständig sichtbar zu machen. 
Für eine niedrige Selbstwirksamkeitsüberzeugung liegen alle Werte im Prinzip unterhalb der blauen Projektionskurve; in dieser Gruppe wären daher durchweg niedrige Leistungen zu erwarten. Zu beachten ist allerdings, dass es sich bei dieser Gruppe wiederum um eine sehr stark gefaltete Katastrophenfläche handelt, sodass grundsätzlich von dieser Gruppe niedriger Selbstwirksamkeitsüberzeugung auch hohe Leistungswerte erreichbar wären ${ }^{144}$.

Für die Gruppe mittlerer Selbstwirksamkeitsüberzeugung liegen bereits einige Messwerte im Bereich oberhalb der grünen Projektionskurve. Aufgrund der stark asymmetrischen Form der zugehörigen Katastrophenoberfläche liegt weiterhin rund der Hälfte der verbleibenden Werte im Bereich des stetigen Oberflächenverlaufes, also dem Bereich von Zustandsänderungen ohne Katastropheneffekte.

Für die Gruppe hoher Selbstwirksamkeitsüberzeugung stellt sich die Situation noch besser dar. Hier liegt die größte Anzahl von Werten oberhalb der roten Projektionskurve, zusätzlich erkennt man jedoch in Abbildung 37, dass auch hier der Übergang von einer Leistungsebene zur anderen durch Katastrophensprünge erfolgt.

Zusammenfassend lässt sich somit feststellen, dass mit zunehmender Selbstwirksamkeitsüberzeugung bessere Leistungen wahrscheinlicher sind. Zusätzlich scheinen in der Untersuchungsgruppe mittlerer Selbstwirksamkeitsüberzeugung Katastropheneffekte zum Teil unterdrückt bzw. von stetigen Leistungsverläufen ersetzt zu sein.

Abschließend erfolgt eine Betrachtung der Sprungerfahrung der Probanden. Abschnitt 5.1.2 zeigte bereits, dass hinsichtlich der Sprungerfahrung Unterschiede im Angst-LeistungsZusammenhang zu erwarten sind. Diese Unterschiede werden zwar noch auf ihre statistische Signifikanz geprüft, zusätzlich sind aber auch für Fallschirmspringer verschiedener Erfahrung abweichende Angst-Leistungs-Oberflächen zu erwarten. Für die in Abschnitt 5.1.2 aus der Zahl von Formationssprüngen und Jahren im Sport gebildeten Gruppen unterschiedlicher relativer Erfahrung wurde wiederum ein Spitzenkatastrophenmodell von Angst und sportlicher Leistung nach Gleichung 8 angepasst.

\footnotetext{
${ }^{144}$ Aufgrund des großen Abstandes der Messwerte von der blauen Projektionskurve scheint dies allerdings recht unwahrscheinlich.
} 
Tabelle 16: Koeffizienten und Fehler der angepassten Spitzenkatastrophenmodelle in den unterschiedlichen Erfahrungsgruppen.

\begin{tabular}{|c|c|c|c|}
\hline & $\begin{array}{c}\text { Niedrige } \\
\text { Erfahrung }\end{array}$ & $\begin{array}{c}\text { Mittlere } \\
\text { Erfahrung }\end{array}$ & $\begin{array}{c}\text { Hohe } \\
\text { Erfahrung }\end{array}$ \\
\hline Pseudo- $\boldsymbol{r}^{\mathbf{2}}$ & $\mathbf{0 , 6 5 7}$ & $\mathbf{0 , 5 5 2}$ & $\mathbf{0 , 8 2 5}$ \\
\hline$\alpha_{\mathbf{1}}$ (SOM) & $0,167(45)$ & $0,197(66)$ & $0,040(6)$ \\
\hline$\alpha_{2}$ (PHYSIO) & $-0,335(61)$ & $-0,012(75)$ & $-0,178(27)$ \\
\hline$\alpha_{3}$ (Konstante) & $-0,000(0)$ & $-0,246(16)$ & $-0,041(10)$ \\
\hline$\beta_{1}$ (KOG) & $-0,212(72)$ & $0,068(41)$ & $0,049(16)$ \\
\hline$\beta_{2}$ (SOM) & $-0,288(52)$ & $-0,290(118)$ & $-0,132(43)$ \\
\hline$\beta_{3}$ (SW) & $-0,022(3)$ & $0,155(58)$ & $0,195(35)$ \\
\hline$\beta_{4}$ (Konstante) & $1,157(155)$ & $1,253(246)$ & $0,756(114)$ \\
\hline$\chi$ (Konstante) & $0,606(68)$ & $0,520(138)$ & $0,325(102)$ \\
\hline
\end{tabular}

Man erkennt vor allem für die Gruppen niedriger und hoher Erfahrung wieder die typische Form der Katastrophenfläche, die in Abbildung 39 graphisch dargestellt ist; Abbildung 40 zeigt die Projektionskurven der Wellenkanten auf die Kontrollebene der Erfahrungsgruppen (blau: niedrige, grün: mittlere und rot: hohe Sprungerfahrung) - der in den Untersuchungen durchlaufene Wertebereich ist grau eingefärbt.

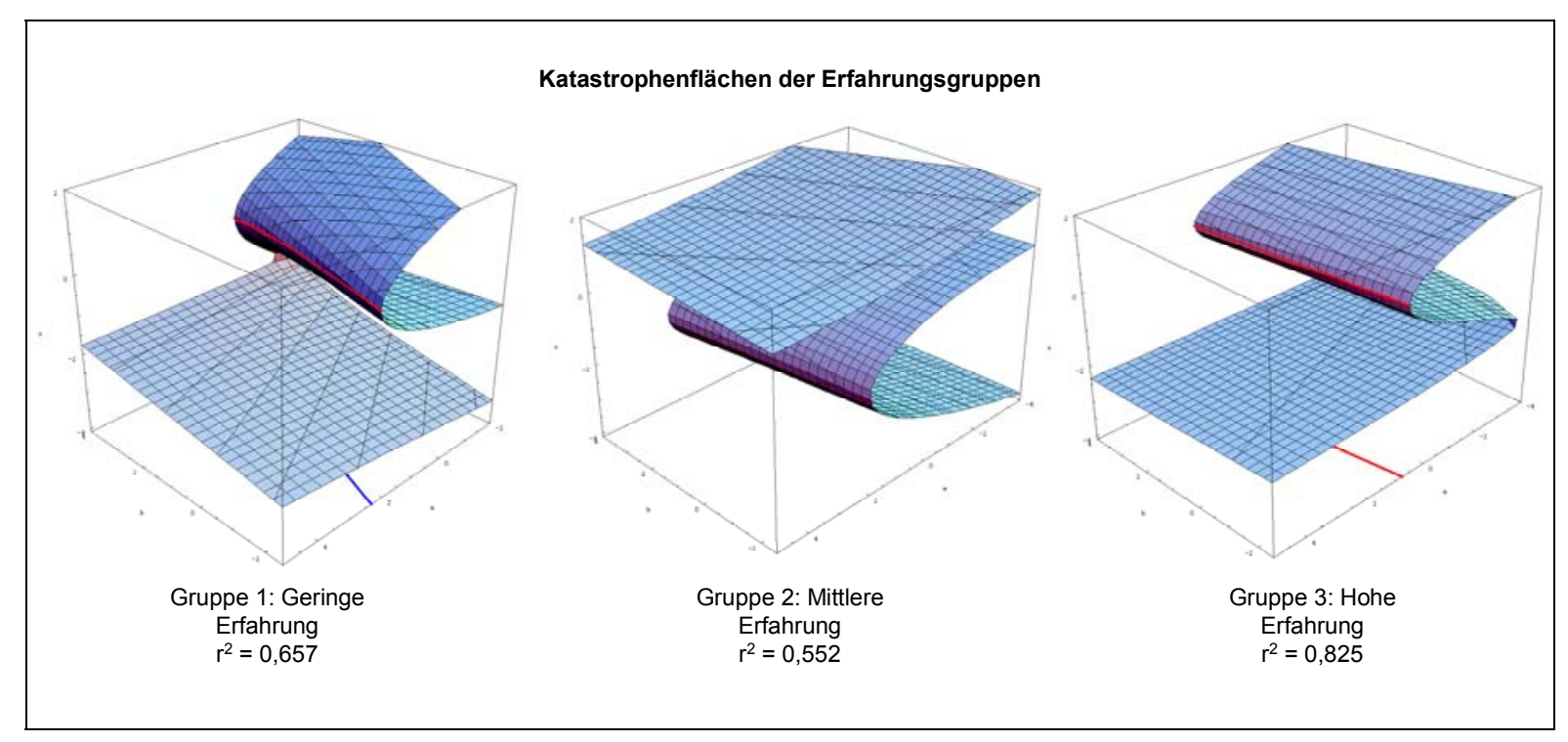




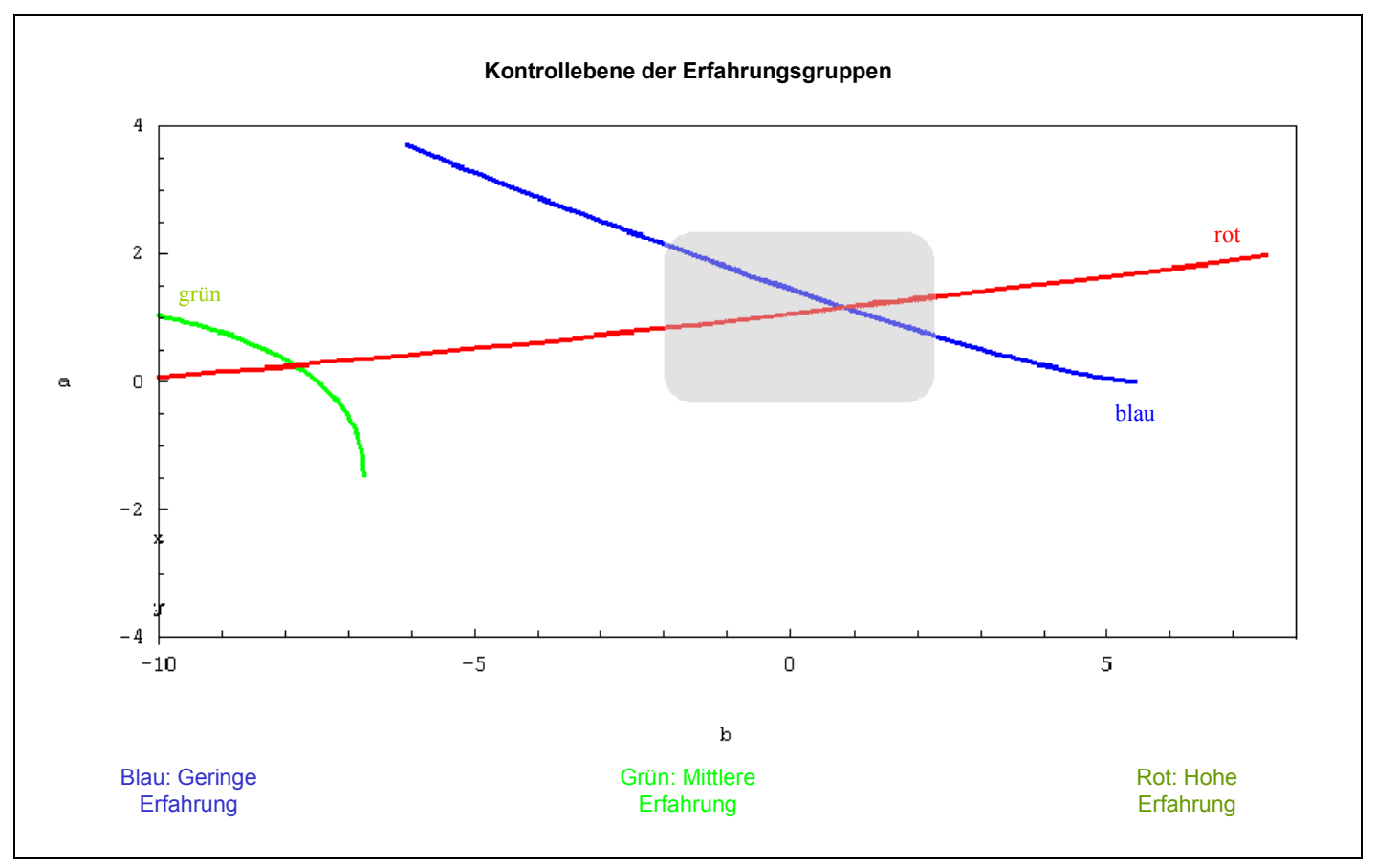

Abbildung 40: Kontrollebene für Gruppen unterschiedlicher Erfahrung.

Bei einer Betrachtung von Abbildung 39 und Abbildung 40 fällt hinsichtlich der Gruppen mit geringer und hoher Erfahrung auf, dass die angepassten Katastrophenflächen in Bezug auf Ort und Faltung der brechenden Welle nahezu identisch sind. In beiden Gruppen extremer Erfahrung liegt jeweils etwa die Hälfte der Messwerte oberhalb bzw. unterhalb der zugehörigen Projektionskurve. Auffallend ist dagegen, dass die Wellenkanten der Gruppen mit geringer und hoher Sprungerfahrung schräg zueinander ausgerichtet sind: In der Gruppe mit geringer Erfahrung führt eine Erhöhung der kognitiven Angstkomponente dazu, dass bei steigender physiologischer Erregung ein Leistungsabfall als „Absturz über die Wellenkante“ ungünstig früh auftritt ${ }^{145}$.

In der Gruppe hoher Sprungerfahrung hat dagegen der Verlauf der roten Projektionskurve in Abbildung 40 eine positive Steigung: In dieser Gruppe treten bei einer gleichzeitigen Erhöhung von kognitiver Angstkomponente und physiologischer Erregung Katastropheneffekte anscheinend später auf als in der Gruppe mit geringer Sprungerfahrung.

\footnotetext{
${ }^{145}$ Die kognitive Angstkomponente ist in Abbildung 40 auf der $b$-Achse (spaltender Faktor) der Kontrollebene dargestellt, die physiologische Erregung auf der $a$-Achse (Normalfaktor). Bei einer Erhöhung des spaltenden Faktors $b$ hat die blaue Projektionskurve eine negative Steigung: Das Auftreten von Katastropheneffekten wird bei steigenden Werten des Normalfaktors $a$ begünstigt.
} 
Somit scheint sich eine steigende Sprungerfahrung der Probanden dadurch auszuwirken, dass ein katastrophales Zusammenbrechen der sportlichen Leistung deutlich verzögert wird. $\mathrm{Zu}$ beachten ist allerdings, dass sich diese beschriebene Tendenz nicht unbedingt in dem Verlauf der Katastrophenoberfläche der Gruppe 2 (mittlere Sprungerfahrung) wiederfinden lässt. Dies mag daran liegen, dass in der Untersuchung nur Gruppen relativer Sprungerfahrung gebildet werden konnten. Zusätzlich erzielt die angepasste Katastrophenoberfläche für diese Gruppe das niedrigste Gütemaß - somit ließe sich eventuell für diese Gruppe eine andere Katastrophenform besser anpassen. Das gezielte Vorgeben einer bestimmten Katastrophenform hinsichtlich Position und Faltung der brechenden Welle ist mit dem Programm GEMCAT II aber leider nicht möglich.

\subsubsection{Hypothesenprüfung}

Die Überprüfung der Hypothesen zeigte folgendes Ergebnis:

H1: Die Gesamtgruppe der Formationsspringer hat im Wettkampf eine signifikant höhere somatische Angstkomponente (Training: $-0,27$; Wettkampf: 0,$08 ; p=0,029$ ) und eine hoch signifikant ${ }^{146}$ größere Herzfrequenz und physiologische Aktivierung (Training: 0,54; Wettkampf: 0,$60 ; p=0,001)$ als im Training.

Der Mittelwert der kognitiven Angstkomponente ist zwar im Wettkampf ebenfalls höher als im Training (Training: $-0,20$; Wettkampf: 0,$06 ; p=0,113$ ) - diese Differenz ist jedoch nicht innerhalb des Signifikanzniveaus.

Damit kann H1 für die somatische Angstkomponente und die physiologische Aktivierung bestätigt werden.

H2: Weder einer der mit den Fragebögen gemessenen Angst- oder Leistungswerte noch die Herzfrequenz bzw. physiologische Aktivierung zeigen signifikante Unterschiede zwischen Sprüngen innerhalb der 4er- oder der 8er-Disziplin.

Damit kann $\mathrm{H} 2$ bestätigt werden.

H3: Die weiblichen Fallschirmspringerinnen gaben signifikant höhere Werte der Eigenschaftsangst (weiblich: STAI 34,7; männlich: STAI 31,8; $p=0,005$ ), kognitiven

\footnotetext{
${ }^{146}$ Für die Signifikanzniveaus wurden jeweils Werte der Irrtumswahrscheinlichkeit $p<0,05$ als signifikant, $p<0,01$ als sehr signifikant und $p<0,001$ als hoch signifikant verwendet (vgl. WILLIMCZIK 1997, 79).
} 
Angstkomponente (weiblich: 0,26; männlich: $-0,11 ; p=0,016)$ und somatischen Angstkomponente (weiblich: 0,21; männlich: $-0,09 ; p=0,050$ ) an.

Für die Selbstwirksamkeitsüberzeugung, die physiologische Aktivierung und die Leistung existieren keine signifikanten Unterschiede.

H3 kann somit für die Eigenschaftsangst sowie die kognitive und somatische Angstkomponente bestätigt werden.

H4: Hinsichtlich des Leistungsniveaus der Formationsspringer existieren signifikante Mittelwertunterschiede zwischen den Gruppen geringer, mittlerer und hoher Sprungerfahrung $^{147}$ bei den Variablen der kognitiven Angstkomponente, der Herzfrequenz und der erbrachten Leistung. So nimmt die kognitive Angstkomponente mit steigender Erfahrung signifikant $\mathrm{ab}$ (gering erfahren: 0,36; mittlere Erfahrung: $-0,07 ; p=0,036$; hoch erfahren: $-0,25 ; p=0,002$ ), ebenso die mittlere Herzfrequenz (gering erfahren: 139,5; mittlere Erfahrung: 131,8; $p=0,004$; hoch erfahren: 130,5; $p=0,001$ ).

Weiterhin erzielten die hoch erfahrenen Fallschirmspringer signifikant bessere persönliche Leistungen als die Springer mit geringer Erfahrung (gering erfahren: $0,29$; hoch erfahren: 0,$21 ; p=0,015)$.

Damit kann H4 für die Leistung, die kognitive Angstkomponente und die Herzfrequenz bestätigt werden.

H5: Die untersuchten Probanden mit einer hohen Eigenschaftsangst haben sehr signifikant größere Werte von kognitiver Angstkomponente (Hochängstliche: 0,64; Niedrigängstliche: $-0,45 ; p=0,000$ ), somatischer Angstkomponente (Hochängstliche: 0,29; Niedrigängstliche: $-0,21 ; p=0,008$ ), Herzfrequenz (Hochängstliche: 137,6; Niedrigängstliche: 129,3; $p=0,001$ ) und physiologischer Aktivierung (Hochängstliche: 0,60; Niedrigängstliche: 0,$56 ; p=0,019)$ als die Probanden mit einer niedrigen Eigenschaftsangst. Die Komponente der Selbstwirksamkeit ist bei den Hochängstlichen kleiner als bei den Niedrigängstlichen (Hochängstliche: $-0,30$; Niedrigängstliche: 0,$13 ; p=0,028$ ). Auch diese Differenz ist statistisch signifikant.

Damit kann H5 für die kognitive und somatische Angstkomponente, die Selbstwirksamkeitsüberzeugung, die mittlere Herzfrequenz und die physiologische Aktivierung bestätigt werden.

\footnotetext{
${ }^{147}$ Siehe Abschnitt 5.1.2 (Seite 112) zur Einteilung der Erfahrungsgruppen.
} 
H6: Probanden mit einer hohen Selbstwirksamkeitsüberzeugung erzielten eine sehr signifikant bessere Leistung (SW hoch: 0,23 ; SW niedrig: $-0,33 ; p=0,007$ ) als solche mit einer niedrigen Selbstwirksamkeitsüberzeugung.

Damit lässt sich $\mathrm{H} 6$ bereits bestätigen.

Berücksichtigt man zudem, dass für das Freifallformationsspringen niedrige Werte von kognitiver und somatischer Angstkomponente als optimal für maximale Leistung vorausgesagt wurden (vgl. Abschnitt 5.2.3.2 auf Seite 83), kann man als erweiterte Hypothesenprüfung auch diese Größen betrachten: Sowohl kognitive (SW hoch: 0,61; SW mittel: $-0,01 ; p=0,000$; SW niedrig: 0,$81 ; p=0,000)$ und somatische Angstkomponente (SW hoch: $-0,41$; SW mittel: $-0,05$; $p=0,000$; SW niedrig: 0,60; $p=0,000$ ) als auch die physiologische Aktivierung (SW hoch: 0,55; SW niedrig: 0,60; $p=0,004)$ sind bei den Probanden mit einer hohen Selbstwirksamkeitsüberzeugung hoch signifikant geringer als bei den Probanden, die in den Fragebögen angaben, niedrigere Werte der Selbstwirksamkeitsüberzeugung zu haben.

Also lässt sich H6 auch für diese Erweiterungen bestätigen.

H7: Die Fallschirmspringer mit einer hohen Leistung im Sprung ${ }^{148}$ hatten eine signifikant niedrigere kognitive Angstkomponente (hohe Leistung: -0,35; niedrige Leistung: 0,18; $p=0,015)$ als solche mit einer niedrigen Leistung. Die somatische Angstkomponente ist zwar auch geringer (hohe Leistung: $-0,18$; niedrige Leistung: 0,$08 ; p=0,367$ ), diese Differenz ist aber nicht signifikant.

Damit kann H7 für die kognitive Angstkomponente bestätigt werden.

H8: Die Gütemaße aller angepassten Katastrophenmodelle sind signifikant höher als die der einfacheren Modelle (vgl. Abschnitte 5.2.1.1 und 5.2.1.3 ab Seite 121).

Ein zusätzlicher direkter Vergleich der quadrierten Abweichungssummen $\phi$ von den Katastrophenmodellen und den konkurrierenden einfachen Modellen ergibt hoch signifikant geringere Abweichungssummen von sämtlichen angepassten Katastrophenflächen gegenüber dem multidimensionalen Angst-Leistungs-Modell nach MARTENS $(\mathrm{Z}=3,30 ; p=0,001)$.

Damit lässt sich $\mathrm{H} 8$ bestätigen.

\footnotetext{
${ }^{148}$ Zur Einteilung von Leistungsgruppen wurde erneut als Kriterium für niedrige Leistung Z-LEISTUNG $<-0,5$ und für hohe Leistung 0,5<Z-LEISTUNG definiert.
} 


\subsection{Diskussion der Ergebnisse}

Das Ziel dieser Untersuchung war die Definition und Überprüfung eines optimalen Erregungsniveaus für das Freifallformationsspringen. Da für die angepassten Katastrophenoberflächen die höchsten Gütemaße und signifikant geringere quadrierte Abweichungssummen $\phi$ (im Vergleich zu konventionellen Modellen) nachgewiesen wurden, ist die Anwendung der Katastrophentheorie zur Quantifizierung eines optimalen Angst- bzw. Aktivierungsniveaus als zulässig anzusehen.

Ein optimaler Erregungszustand ist in den angepassten Katastrophenflächen überall dort zu finden, wo die obere Leistungsebene erreicht wird - vorzugsweise sollten dabei zusätzlich von den Kontrollvariablen Werte außerhalb der charakteristischen Spitzenprojektionen angenommen werden, damit keine Katastropheneffekte auftreten können.

Wie erwartet ist das Erreichen der oberen Leistungsebene mit niedrigen Werten von kognitiver Angstkomponente und physiologischer Aktivierung verknüpft. Der kognitiven Angstkomponente kommt als spaltender Faktor $b$ im Prinzip eine Sonderrolle zu: Da der spaltende Faktor, wenn er klein genug ist, das Auftreten von Katastropheneffekten verhindern kann, sollte für ein optimales Erregungsniveau besonders die kognitive Angstkomponente gering sein.

Eine weitere Forderung für eine optimale Erregung ist, dass die physiologische Aktivierung bzw. die somatische Angstkomponente ebenfalls gering sein sollten. Aus dem Verlauf der Herzfrequenz ergibt sich jedoch, dass die physiologische Aktivierung während eines Fallschirmsprungs nicht niedrig sein kann, denn die Herzfrequenz steigt unter allen Umständen bei jedem Absprung stark an. Somit hat es den Anschein, dass die physiologische Aktivierung nur in begrenztem Maße steuerbar ist. Aus diesem Grund zeigte die Überprüfung von $\mathrm{H} 7$ vermutlich auch, dass gute Leistungen nur mit niedrigen Werten der kognitiven Angstkomponente verknüpft sind.

Dieses unvermeidliche drastische Steigen der physiologischen Aktivierung im Moment des Absprungs kann sich weiterhin durchaus negativ auswirken: Ein Formationsspringer mit einer höheren kognitiven Angstkomponente wird sich aus katastrophentheoretischer Sicht in dem Bereich der gebogenen Leistungsoberfläche befinden, in dem Katastropheneffekte auftreten können - ohne sich womöglich dessen bewusst zu sein. Obwohl sich der Springer zunächst noch im Bereich der oberen Leistungsfläche befindet, kann es nun sein, dass durch das 
Anwachsen der physiologischen Aktivierung nach dem Absprung der „Absturz“ auf die schlechte Leistungsfläche erfolgt. Somit lässt sich z.B. der plötzliche (und in der Regel völlig unerwartete) „Brainlock“149 erklären (vgl. SCHIEDEK 2000, 11).

Der Angst-Leistungs-Zusammenhang (und damit auch ein optimales Erregungsniveau) wird durch zusätzliche Größen beeinflusst, die in Abschnitt 3.2 (ab Seite 35) erläutert wurden:

Hinsichtlich des Geschlechts der Probanden konnte nachgewiesen werden, dass Frauen höhere Werte der Eigenschaftsangst sowie der kognitiven und somatischen Angstkomponente während der beobachteten Fallschirmsprünge aufwiesen als die männlichen Probanden. Diese Unterschiede waren nach der Theorie zu erwarten. Es stellt sich allerdings die Frage, ob die Frauen, die sich entscheiden, den Fallschirmsport auszuüben, in der Tat eine höhere Ängstlichkeit als Männer aufweisen ${ }^{150}$ oder ob es sich hierbei nur um die bereits in Abschnitt 3.2.1.4 auf Seite 39 diskutierten Unterschiede hinsichtlich einer größeren Ehrlichkeit der Frauen beim Ausfüllen der Fragebögen handelt.

Der Erfahrungsstand der Probanden ist eine weitere Größe, die den Angst-LeistungsZusammenhang moderiert: Hoch signifikante Vorteile von sehr erfahrenen gegenüber weniger erfahrenen Formationsspringern bezüglich der Leistung, der physiologischen Aktivierung, der kognitiven Angstkomponente und dem Verlauf der angepassten Katastrophenoberflächen hinsichtlich eines späteren Auftretens von Katastropheneffekten waren nachweisbar. Wie zuvor aufgeführt, ist dabei die besondere Rolle der kognitiven Angstkomponente zu beachten: Niedrige Werte der kognitiven Angstkomponente sind in der Untersuchung mit besseren Leistungen verknüpft: Sprung- und Wettkampferfahrung reduziert bei den betrachteten Fallschirmspringern diese Angstkomponente und scheint die individuell bewertete erreichte Leistung zu erhöhen.

Weiterhin konnte gezeigt werden, dass der für das Freifallformationsspringen optimale Erregungszustand in den Gruppen unterschiedlicher Eigenschaftsangst verschieden ist. Die Gruppe der Probanden mit einer niedrigen Eigenschaftsangst hat den größten Anteil von Versuchswerten außerhalb der Region, in der Katastropheneffekte auftreten (vgl. Abbildung

\footnotetext{
${ }^{149}$ Vgl. Abschnitt 5.2.3.

${ }^{150}$ Und aufgrund dieser höheren Eigenschaftsangst dann auf die angstauslösende Situation des Fallschirmsprunges auch mit größeren Angstwerten reagieren (siehe Abschnitt 1.1.2 auf Seite 6).
} 
36); im Angst-Leistungs-Zusammenhang der beiden anderen Ängstlichkeitsgruppen können Katastrophenereignisse nicht vermieden werden. Anhand der sehr ähnlichen Form der in den Gruppen unterschiedlicher Eigenschaftsangst angepassten Katastrophenoberflächen lässt sich die Vermutung aufstellen, dass in einem Katastrophenmodell von Angst und sportlicher Leistung die verschiedene Ängstlichkeit der Probandengruppen eine reine Verschiebung der Wellenformen in Richtung des Normalfaktors $a$ bewirkt.

Bei einer Betrachtung der zusätzlichen Gruppen unterschiedlicher Selbstwirksamkeitsüberzeugung konnte die besondere Rolle dieser Messgröße bestätigt werden. Eine hohe Selbstwirksamkeitsüberzeugung ist mit besseren Leistungen verknüpft und schützt anscheinend nachhaltig vor dem Eintreten von Katastropheneffekten. Somit ist als Erweiterung eines optimalen Erregungsniveaus für das Freifallformationsspringen zu definieren, dass eine hohe Selbstwirksamkeitsüberzeugung anzustreben ist.

Die Messgröße der Selbstwirksamkeitsüberzeugung konnte weiterhin auch durch ein Schmetterlingskatastrophenmodell in die Beschreibung des Angst-Leistungs-Zusammenhangs integriert werden. Für eine besondere Rolle der Selbstwirksamkeit sprechen die außerordentlich hohen Gütemaße, die in den Gruppen unterschiedlicher Eigenschaftsangst bei der Anpassung der Schmetterlingskatastrophen erzielt wurden.

$\mathrm{Ob}$ diese Gütemaße aber wirklich verlässlich sind und ob doch eine graphische Interpretation von Messdaten im Rahmen der Schmetterlingskatastrophe möglich ist, konnte nicht geklärt werden. Aus diesem Grund wurde die Entscheidung gefällt, Spitzenkatastrophenmodelle in den Gruppen mit unterschiedlicher Selbstwirksamkeitsüberzeugung anzupassen. Auf diese Art und Weise konnte die besondere Rolle der Selbstwirksamkeit durch den Vergleich der Katastrophenflächen indirekt in das Modell der Spitzenkatastrophe integriert werden.

Somit bleibt als einziger Nachteil der Spitzenkatastrophe, dass diese nur bimodale Leistungsdaten beschreiben kann und nicht, wie die Schmetterlingskatastrophe, Trimodalität zulässt.

Es ist zu vermuten, dass ein rein differentialpsychologischer Ansatz den Zusammenhang von Angst und sportlicher Leistung noch besser beschreiben kann. Keine der mir bekannten Arbeiten, die den Angst-Leistungs-Zusammenhang erforschen, nutzte diese Herangehensweise. In dieser Arbeit wurde wenigstens partiell, durch die Einteilung der Probanden in Gruppen gleicher Eigenschaftsangst, auf die Persönlichkeitsunterschiede der untersuchten Fallschirmspringer eingegangen. 
Die Gütemaße der erklärten Leistungsvarianz waren womöglich auch dadurch in dieser Untersuchung höher als die in den neueren Untersuchungen von HARDY et al. (1996) und PAIN (1999), die ebenfalls Katastrophenmodelle zur Beschreibung des Angst-LeistungsZusammenhangs nutzten. Dabei mag sich die Wahl der Gruppeneinteilung bezüglich der Eigenschaftsangst als glücklich erwiesen haben, ermöglichte sie doch das Sammeln hinreichend großer Datenmengen innerhalb der Ängstlichkeitsgruppen in einem relativ kurzen Untersuchungszeitraum. Ein von COHEN et al. (2003) empfohlener rein differentialpsychologischer Ansatz, der eine Beeinflussung der Zustandsangst durch weitere Persönlichkeitsmerkmale (wie z.B. Angstbewältigungsstrategien, Risikobereitschaft etc.) berücksichtigen kann, hat jedoch den Nachteil, dass eine sehr lange Zeitspanne eingeplant werden muss, um die für die Anpassung eines Katastrophenmodells erforderliche Datenmenge für jede einzelne Testperson zu erhalten. Durch diesen langen benötigten Zeitraum wäre es vermutlich sehr schwierig, reine Wettkampfangst zu messen - wichtige Wettbewerbe im Fallschirmsport finden nur einmal pro Jahr statt und beinhalten nur maximal 10 Runden (Sprünge).

Wünschenswert wäre allerdings ein differentialpsychologischer Ansatz bei einer Bestätigung der Eigenschaftsangst als Faktor, der eine Verschiebung der Wellenkanten in Katastrophenmodellen von Angst und sportlicher Leistung zu bewirken scheint.

Die Untersuchung zeigte ferner aus physiologischer Sicht, dass der gemessene Verlauf der Herzfrequenz im Moment des Absprungs deutlich zunimmt. Dies liegt zum Einen an der Ausschüttung von Adrenalin und Noradrenalin ins Blut, die ein Ansteigen der Herzfrequenz bewirken (vgl. Abschnitt 1.2.2 auf Seite 10). Dieser Herzfrequenzanstieg aufgrund von Hormonausschüttung betrifft anscheinend auch den „Experten“ im Fallschirmsport.

Zum Anderen beginnt natürlich im Freifall die aktive Bewegung des Körpers, die ebenfalls eine Zunahme der Herzfrequenz zur Folge hat. In welchem Größenverhältnis diese Parameter die Herzfrequenz beeinflussen, ist nicht bekannt. Rотн et al. versuchten 1996 den Einfluss der sportlichen Bewegung auf die Herzfrequenz beim Fallschirmsprung zu messen, indem sie die Herzfrequenz auch bei Absprungübungen am Boden ermittelten und von diesem Wert auf die wahre Einflussgröße schlossen (vgl. Rотн et al. 1996, 65).

Dieses Vorgehen ist bei der Betrachtung von Formationssprüngen im Freifall jedoch nicht möglich. Zwar wird auch hier der Sprungablauf am Boden, auf Rollbrettern liegend, geübt, jedoch dienen diese Übungen im Prinzip nur der Visualisierung der Formationen und einer Vorwegnahme der zu fliegenden Wege. Die reine körperliche Bewegung ist bei dieser Art des Trainierens aber eine völlig andere. 
Einzige Möglichkeit, Aussagen über die bewegungsbezogene Steigerung der Herzfrequenz wäre die Beobachtung von simulierten Formationssprüngen in einem vertikalen Windtunnel ${ }^{151}$, wie er u.a. heutzutage zur Unterstützung im Hochleistungstraining von Formationsspringern eingesetzt wird. In einem solchen Windtunnel wären die Bewegungen des freien Falls simulier- und messbar, ohne dass gleichzeitige Angstreaktionen zu erwarten sind schließlich handelt es sich hier nicht um eine Situation körperlicher Gefährdung. Vielleicht kann eine solche Untersuchung in späteren Arbeiten realisiert werden.

Weiterhin weicht der gemessene Verlauf der Herzfrequenz bei Freifallformationsspringern von dem durch Fenz/EPSTEIN (1967) und REID et al. (1971) beschriebenen Verlauf ab. Beide beschrieben Unterschiede im qualitativen Verlauf zwischen Anfängern und Experten im Sport. Diese Unterschiede waren selbst im Vergleich der Herzfrequenzverläufe zwischen einzelnen Probanden in dieser Untersuchung nicht reproduzierbar. Dies mag natürlich daran liegen, dass die unerfahrenen Springer dieser Untersuchung bereits eine Sprungzahl aufwiesen, die bei FENZ/EPSTEIN sowie bei REID et al. bereits den Experten definierte.

Beide dieser älteren Untersuchungen beschrieben allerdings den Moment der Fallschirmöffnung als den Zeitpunkt der höchsten Herzfrequenz im Sprungablauf, dicht gefolgt von der Landung. Auch diese Aussage konnte in der vorliegenden Untersuchung nicht reproduziert werden: Zwar kann man in Abbildung 27 und Abbildung 28 auf Seite 109 im Verlauf der Herzfrequenz zum ungefähren Zeitpunkt der Fallschirmöffnung eine Spitze erkennen, diese ist jedoch nicht so ausgeprägt wie von FENZ/EPSTEIN beschrieben. Ein deutliches Ansteigen der Herzfrequenz vor der Landung ${ }^{152}$ ist bei einer Betrachtung der Gesamtgruppe nicht erkennbar: In Einzelfällen hat die Herzfrequenzkurve ein eher unscheinbares Nebenmaximum zum Zeitpunkt der Landung.

Diese Abweichungen sind mit großer Wahrscheinlichkeit durch die veränderte Fallschirmtechnik seit den älteren Untersuchungen zu begründen. Mit der ausschließlichen Verbreitung von voll steuer- und bremsbaren Flächenfallschirmen ab Mitte der 80er Jahre ist sowohl das Öffnungsverhalten eines Fallschirms sicherer als auch der Landungsvorgang einfacher und mit weniger Verletzungsrisiko verknüpft geworden (vgl. SCHEDLOWSKI/TEWES 1992, 101).

\footnotetext{
${ }^{151}$ Hierbei handelt es sich um einen Windtunnel, der Luft mit Geschwindigkeiten von bis zu 220km/h von unten nach oben „bläst“. In einem so erzeugten Luftstrom können Freifallmanöver sehr realistisch simuliert werden.

152 Die Flugzeit der Probanden am geöffneten Fallschirm betrug zwischen 1,2 und 3,1 Minuten. Somit erfolgte die Landung in Abbildung 27 und Abbildung 28 auf Seite 109 in einem Zeitintervall von etwa 130 bis 230 Sekunden (4er Disziplin) bzw. 150 bis 250 Sekunden (8er Disziplin) nach dem Exit.
} 
Ein Blick gilt noch den beobachteten Korrelationen zwischen den einzelnen Messgrößen des verwendeten CSAI-2-Angstfragebogens. Zum Einen erscheint es natürlich selbstverständlich, dass in einer Nicht-Labor-Situation wie dem Fallschirmspringen in Momenten hoher kognitiver Angst auch die somatische Angstkomponente erhöht ist. Diese (geringen) Korrelationen lassen sich auf nahezu ideale Art in der Anpassung von Katastrophenflächen mit dem Programm GEMCAT II berücksichtigen, da eine Messgröße zu beiden Kontrollvariablen der Spitzenkatastrophe beitragen konnte. Auf diese Art und Weise war es möglich, diese Korrelationsanteile „herauszufiltern“.

Zum Anderen ist aber eine Korrelation zwischen kognitiver Angstkomponente und Selbstwirksamkeitsüberzeugung zwar begründbar, da beide nach MARTENS et al. (1990) die entgegengesetzten Enden einer kognitiven Skala darstellen (vgl. PARFITT et al. 2000, 47; MARTENS et al. 1990, 24), jedoch stellt sich die Frage, ob nicht Zusammenhänge doppelt erfasst werden, wenn die beiden Größen hochgradig korrelieren. Es ist aber zu bedenken, dass kognitive Angstkomponente und Selbstwirksamkeitsüberzeugung trotz einer möglichen Korrelation den Angst-Leistungs-Zusammenhang unterschiedlich moderieren (vgl. EDWARDS et al. 2002, 11). Weiterhin haben beide Größen in einem Katastrophenmodell von Angst und sportlicher Leistung unterschiedliche Funktionen - man beachte zusätzlich die besondere Rolle der Selbstwirksamkeitsüberzeugung als Messgröße, die anscheinend vor dem Auftreten von Katastropheneffekten zu schützen vermag.

Das in dieser Arbeit untersuchte optimale Erregungsniveau bezieht sich zunächst nur auf Freifallformationsspringer. Zumindest im Rahmen der Freestyle-, Skysurf- und FreeflyingDisziplin des Fallschirmsports ist aber mit sehr ähnlichen komplexen kognitiven und motorischen Anforderungen zu rechnen, die eine Übertragung der gefundenen Ergebnisse möglich erscheinen lassen. 


\section{Praxisbezogene Relevanz der Ergebnisse}

Alle Probanden dieser Untersuchung waren bereits fertig ausgebildete Fallschirmspringer mit einem unterschiedlichen Erfahrungsgrad im Freifallformationsspringen. Aus den Ergebnissen dieser Arbeit lassen sich einige Forderungen zum Erreichen eines optimalen Erregungsniveaus für das Freifallformationsspringen aufstellen. Diese Forderungen gehen zunächst von einem Ansatz der Angstmessung aus, wie er in dieser Arbeit verwendet wurde ${ }^{153}$. Es sollte aber auch möglich sein, einen Sportler zu schulen, seinen momentanen Zustandsangstwert direkt auf einer Skala anzugeben (vgl. HARDY 1996a, 74; STAFFORD 1996, 33; METNI 2001).

1. Die besondere Rolle der kognitiven Angstkomponente als die Kontrollgröße, die über das Eintreten von Katastropheneffekten entscheidet, sollte verdeutlicht werden. Die kognitive Angstkomponente zeigte in dieser Untersuchung einen starken Zusammenhang mit der sportlichen Leistung; niedrige Werte dieser Angstkomponente sind anzustreben $^{154}$. Weiterhin besteht der Verdacht, dass die kognitive Zustandsangst die einzige leicht zu manipulierende Größe ist, da die physiologische Erregung in der konkreten Situation des Fallschirmspringens durch die Hormonausschüttungen nicht kontrollierbar ist. Ein zusätzliches Ausdauertraining könnte es ermöglichen, die physiologische Erregung ,indirekt“ über die Abnahme des Ruhepulses zu verringern.

2. Hohe Werte der Selbstwirksamkeitsüberzeugung beeinflussen die Leistung beim Freifallformationsspringen anscheinend positiv und können vor dem Eintreten von Katastropheneffekten schützen. Somit ist es sinnvoll, ein Gefühl der Selbstsicherheit schon in der Sprungvorbereitung zu provozieren. Sprünge sollten bereits unter dem Gesichtspunkt geplant werden, dass jeder Sportler in hohem Maße von seiner Fähigkeit überzeugt ist, die geplanten Bewegungen optimal ausführen zu können (vgl. JEFFRIES 1997; MALONE 2001, 24; BIEDERMANN 2002, 106f).

Diesem Gedanken kommt während der Vorbereitung von Wettkampfsprüngen eine besondere Bedeutung zu: Bei aufeinanderfolgenden Wettkampfrunden beträgt die minimale Zeitspanne, die eine Mannschaft zwischen der Landung und dem Besteigen des Flugzeuges für die nächste Wettbewerbsrunde hat, eine Stunde (vgl. FAI im

\footnotetext{
${ }^{153}$ Messung der Eigenschaftsangst mit dem STAI, der Zustandsangst mit dem CSAI-2 und der physiologischen Aktivierung über die Herzfrequenz.

154 Z.B. durch die Nutzung von mentalem Training oder speziellen Angstbewältigungsstrategien (vgl. DeRosalia 2000, 99f; WiLliams/Harris 1998, 225ff; BiedERMANN 2002, 92f).
} 
Internet). Somit ist die effektiv für die Sprungvorbereitung zur Verfügung stehende Zeit mitunter sehr kurz ${ }^{155}$ - dieser Zeitmangel darf aber nicht $\mathrm{zu}$ einer übereilten Sprungvorbereitung führen: Die Planung eines Wettkampfsprunges ist erst dann abgeschlossen, wenn jeder beteiligte Springer davon überzeugt ist, die geforderten Bewegungen perfekt ausführen zu können.

3. Während der Vorbereitung auf einen Sprung im Steigflug mit dem Flugzeug sollte ein bewusstes Verstärken selbstsicherer Stimmungen und Gefühle und eine Reduktion der Besorgnis, also der kognitiven Angstkomponente, erfolgen.

Ein solches Verstärken der Selbstwirksamkeitsüberzeugung wird im Moment von nur $17 \%$ der in diesen Untersuchungen getesteten Probanden im Rahmen der Sprungvorbereitung durchgeführt, 87\% konzentrieren sich ausschließlich auf den zu erwartenden Sprungverlauf.

4. Fallschirmspringer sollten bereits in der Anfänger- und Grundlagenausbildung mehr sensibilisiert werden, den eigenen Körper in sämtlichen Phasen des Fallschirmsprungs bewusst wahrzunehmen. Auf diese Art und Weise könnten Messfehler aufgrund mangelnder Selbstwahrnehmung der Körperreaktionen vermieden werden: Nur wenn die beteiligten Sportler ein genaues Bild ihrer eigenen Körperreaktionen in der Angstsituation des Fallschirmspringens haben, ist es möglich, gezielt bestimmte Erregungszustände anzusteuern. Gespräche mit zahlreichen an diesen Untersuchungen beteiligten Probanden belegen, dass in diesem Punkt der Ausbildung von Fallschirmspringern starke Defizite bzw. anderweitig definierte Prioritäten vorhanden sind.

Vor dem Hintergrund der Tatsache, dass diese kritisierte mangelhafte Selbstwahrnehmung ihre Ursache auch in bereits erfolgender Angstbewältigung haben kann, kann und soll das Ziel der verstärkten Sensibilisierung allerdings nicht sein, hinsichtlich der somatischen Erregung ähnlich hohe Maxima wie die der Herzfrequenz zu erzwingen. Vielmehr sollen die Sportler durch eine verbesserte Körperwahrnehmung in die Lage versetzt werden, Erregungszustände gezielt ansteuern zu können.

\footnotetext{
${ }^{155}$ In dieser Stunde müssen die Fallschirme für den nächsten Sprung gepackt werden, der nächste Sprung geplant sowie sonstige persönliche und Mannschaftsroutinen der Vorbereitung auf den nächsten Fallschirmsprung absolviert werden.
} 


\section{Zusammenfassung und Ausblick}

In der vorliegenden Arbeit wurde der Zusammenhang von Angst und sportlicher Leistung kombiniert mit der Einführung in die Katastrophentheorie und einer Demonstration der Anwendbarkeit dieser Theorie, um den Angst-Leistungs-Zusammenhang besser beschreiben zu können, behandelt.

Die Untersuchungsergebnisse ergaben, dass mit Hilfe des Katastrophenmodells der größte Anteil der gemessenen Leistungsvarianz bei Freifallformationsspringern erklärbar ist. Von diesen Katastrophenmodellen ausgehend, waren qualitative und quantitative Aussagen über ein optimales Erregungsniveau für das Freifallformationsspringen - aufgeteilt in Gruppen unterschiedlicher Eigenschaftsangst - möglich. Bei einem Vergleich dieser Gruppen scheint der Ängstlichkeit die besondere Rolle eines Faktors einzunehmen, der die Katastrophenoberflächen des Zusammenhangs von Angst und sportlicher Leistung in Richtung des Normalfaktors $a$ nahezu parallel verschiebt. Katastropheneffekte wie plötzliches Zusammenbrechen der sportlichen Leistung, Bimodalität der Leistungswerte und Hystereseeffekte im zeitlichen Leistungsverlauf wären demnach bei Hochängstlichen wahrscheinlicher als bei Niedrigängstlichen.

Ferner wurde in den Untersuchungen ein besonderer Einfluss von kognitiver Angstkomponente, Selbstwirksamkeitsüberzeugung und Leistungsniveau bzw. Sprungerfahrung der Probanden deutlich. Diese Faktoren konnten zwar mittels der Form einer Schmetterlingskatastrophe in das Katastrophenmodell von Angst und sportlicher Leistung integriert werden - der klare Nachweis dieses Modells bleibt indes aufgrund der hohen Komplexität noch offen, auch wenn die in dieser Untersuchung genäherten Gütemaße der Schmetterlingskatastrophe sehr Erfolg versprechend erscheinen. Die Anpassung von weniger komplexen Spitzenkatastrophenflächen in den Gruppen unterschiedlicher Selbstwirksamkeit und Sprungerfahrung belegte jedoch eindeutig, dass große Werte der Selbstwirksamkeitsüberzeugung und eine hohe Sprungerfahrung mit niedrigeren kognitiven Angstwerten, besseren Leistungen und dem teilweise späteren Auftreten negativer Katastropheneffekte verknüpft sind.

Im Rahmen dieser Untersuchungen wurde - meines Wissens nach erstmals - versucht, einen wenigstens partiell differentialpsychologischen Ansatz bei der Beschreibung des AngstLeistungs-Modells mit der Katastrophentheorie zu nutzen. Dabei mussten einige Näherungen und Verallgemeinerungen in Kauf genommen werden. Eine von COHEN et al. (2003) empfohlene rein differentialpsychologische Untersuchung, die weitere Persönlichkeits- 
faktoren berücksichtigt, ist mit Sicherheit mit einem noch größeren als dem hier betriebenen Aufwand verbunden.

Eine einzige Möglichkeit der Betrachtung des einzelnen Sportlers wäre durch eine Langzeitbeobachtung oder durch eine Reduktion auf die IZOF-Methode von HANIN gegeben. Eine Definition des optimalen Erregungsniveaus für den einzelnen Freifallformationsspringer nach der IZOF-Methode wird von METNI (2001, im Internet) vorgeschlagen. Diese Methode hat jedoch den großen Nachteil, dass sie ausschließlich der quantitativen Identifikation eines individuellen optimalen Erregungsniveaus dienen kann - sie vermag jedoch nicht, Zusammenhänge im Sinne einer eigenen Angst-Leistungs-Theorie zu erklären.

Aufgrund der teilweise sehr ähnlich erscheinenden Aufgaben- und Bewegungsstruktur der „neueren“ Freifalldisziplinen Freestyle, Skysurfen und Freefly ist anzunehmen, dass sich die Untersuchungsergebnisse auf diese Teildisziplinen des Fallschirmsports übertragen lassen. Eine Erweiterung und Überprüfung dieser Ergebnisse innerhalb der anderen Fallschirmsportdisziplinen wie Stil- und Zielspringen wäre jedoch anzustreben. 


\section{Literatur}

Anshel, M.H./Williams, L.R.T./Williams, S.M. (2000): Coping Style Following Acute Stress in Competitive Sport.

In: The Journal of Social Psychology 140, S. 751-773.

Anshel, M.H./Williams, L.R.T./Hodge, K. (1997): Cross-Cultural and Gender Differences on Coping Style in Sport.

In: Journal of Sport Psychology 28, S. 141-156.

Allmer, H. (1981): $\quad$ Psychologische Aspekte sportlicher Beanspruchung.

In: NitsCH, J.H. (Hg.): Streß: Theorien, Untersuchungen, Maßnahmen (S. 503-545). Bern.

Anfilogoff, R./Hale, P.J./Nattrass, M. (1987): Physiological response to parachute jumping. In: British medical journal 295, S. 415.

APITZSCH, E. (1983): $\quad$ An alternative approach to the measurement of anxiety in sports. In: JANSSEN, J.P./HAHN, E. (Hg.): Aktivierung, Motivation, Handlung und Coaching im Sport (S. 111-113). Schorndorf.

ARNOLD, V.I. (1999): $\quad$ Bifurcation theory and catastrophe theory. Berlin.

AsendorPf, J.B. (1996): Psychologie der Persönlichkeit. Berlin.

BAR-DAYAn, Y./Shemer, J. (1998): Parachuting injuries: A retrospective study of 43.542 military jumps. In: MilitaryMedicine 163, S. 1-2.

BERGAN, P. (2000): $\quad$ World championships of formation skydiving statistics 19851999. Oslo.

BERLYNE, D.E. (1974): Konflikt, Erregung, Neugier. Stuttgart.

BIEDERMANN, M. (2002): Leistungsfördernde Faktoren.

In: Biedermann, M./Rehmer, S./Schilling, G.: Psychische Aspekte der sportlichen Leistung (S. 79-126). Zürich. 
Biscoe, N.J. (1999): $\quad$ Coping with Fear. In: Skydive: The Mag Heft 10/1999, S. 10-13.

BÖCKHELER, J.F. (1995): $\quad$ Fear or excitement - a matter of experience.

In: European Review of Applied Psychology 45, S. 83-91.

Bös, K./Mechling, H. (1983): Dimensionen sportmotorischer Leistungen. Schorndorf.

BORCHARDT, M. (1981): Über die Anwendbarkeit der Katastrophentheorie zur Beschreibung und Erklärung psychologischer Sachverhalte. Göttingen.

BreIVIK, G. (1999): $\quad$ Personality, sensation seeking and risk taking among top level climbers, parachute jumpers and white water kayakers.

In: BREIVIK, G. (Hg.): Empirical studies of risk sports (S. 8-26). Oslo.

BreiviK, G./Roth, W.T./Jørgensen, P. (1998): Personality, psychological states and heart rate in novice and expert parachutists.

In: Personality and Individual Differences 25, S. 365-380.

Burton, D. (1988): Do anxious swimmers swim slower? Reexamining the elusive anxiety-performance relationship.

In: Journal of Sport and Exercise Psychology 10, S. 45-61.

Clancy, R. (1999): $\quad$ Fly Higher. Psychology Skills for Peak Performance. A Skydivers' Handbook. Sydney.

Clarkson, M. (1999): Competitive fire. Champaign.

Cohen, A./Pargman, D./Tenenbaum, G. (2003): Critical Elaboration and Empirical Investigation of the Cusp Catastrophe Model: A Lesson for Practitioners.

In: Journal of Applied Sport Psychology 15, S. 144-159.

Combs, A. (1995): $\quad$ Psychology, Chaos, and the Process Nature of Consciousness. In: ABRAHAM, F.D./Gilgen, A.R. (Hg.): Chaos theory in psychology (S. 129-138). Westport. 
Deinzer， R./Kirschbaum， C./Gresete，C./Hellhauer， D.H. (1997): Adrenocortical Responses to Repeated Parachute Jumping. In: Physiology \& Behaviour 61, S. 507-511.

Deroanne, R./Cession-Fossion, A./Juchmes, J./Servais, J.C./Petit, J.M. (1975): Telemetric control of heart adaption during automatic and freefall parachute jumps.

In: Aviation, Space and Environmental Medicine 46, S. 128-131.

DeRosalia, J.J. (2000): Mental Training for Skydiving and Life. West Hurley.

DeUtSCHER FALlsChiRMSPORTVERBAND (DFV) E.V.: Disziplinen im Fallschirmsport. Internet: http://www.fallschirmsportverband.de. 09/2003.

D’Urso, V./Petrosso, A./Robazza, C. (2002): Emotions, Perceived Qualities, and Performance of Rugby Players.

In: The Sport Psychologist 16, S. 173-199.

DURTSCHI, S.K. (2001): Emotions and cognitions of athletes competing in a high-risk sport. Eugene.

Edwards, T./Hardy, L./Kingston, K./Gould, D. (2002): A Qualitative Analysis of Catastrophic Performances and the Associated Thoughts, Feelings, and Emotions.

In: The Sport Psychologist 16, S. 1-19.

EDWARDS, T./HARDY, L. (1996): The interactive effects of intensity and direction of cognitive and somatic anxiety and self confidence upon performance. In: Journal of Sport and Exercise Psychology 18, S. 296-312.

Eide, R./AtterÅs, A. (1978): Blood Glucose. In: Ursin, H./BAAde, E./LeVine, S. (Hg.): Psychobiology of Stress. A Study of Coping Men (S. 99-103). New York.

ElerT, G. (2000): Measuring Chaos. The Lyapunov Exponent and the Lyapunov Space. Internet: http://hypertextbook.com/chaos 03/2003. 
Ender, N.S./Crooks, D.S./PARKer, J.D. (1992): The interaction model of anxiety: An empirical test in a parachute training situation. In: Anxiety, Stress and Coping 5, S. 301-311.

Engels, H.J./Zhu, W./Moffatt, R.J. (1998): An Empirical Evaluation of the Prediction of Maximal Heart Rate. In: RQES 69, S. 94-98.

Eubank, M./Collins, D. (2000): Coping with pre- and in-event fluctuations in competitive state anxiety: A longitudinal approach. In: Journal of Sports Sciences 18, S. 121-131.

FALK, B./BAR-Eli, M. (1995): The Psycho-Physiological Response to Parachuting Among Novice and Experienced Parachutists.

In: Aviation, Space \& Environmental Medicine 66, S. 114-117.

FÉdÉrAtion AÉronautiQue Internationale (FAI): International FAI Parachuting Commission (IPC).

Internet: http://www.fai.org/parachuting. 03/2003.

FenZ, W.D./Epstein, S. (1967): Gradients of Physiological Arousal in Parachutists as a Function of an Approaching Jump.

In: Psychosomatic Medicine 29, S. 33-51.

FENZ, W.D./JONES, G.B. (1972): Individual differences in physiological arousal and performance in sport parachutists.

In: Psychosomatic Medicine 34, S. 1-8.

Fox, S.M./NAughton, J.P./Haskell, W.L. (1971): Physical activity and the prevention of coronary heart disease.

In: Annals of Clinical Research 3, S. 404-432.

FreBer, J./SchMID, M. (1987): Instabilität und Dynamik. Zur Anwendung der Katastrophentheorie in der Soziologie. Neubiberg.

GAL-OR, Y./TENENBAUM, G./FurST, D. (1985): Effect of self-control and anxiety on training performance in young and novice parachuters.

In: Perceptual and Motor Skills 60, S. 743-746. 
GiACOBBI, P.R./WeINBERG, R.S. (2000): An Examination of Coping in Sport: Individual Trait Anxiety Differences and Situational Consistency. In: The Sport Psychologist 14, S. 42-62.

GiLgen, A.R. (1995): $\quad$ A Search for Bifurcations in the Psychological Domain. In: ABRAHAM, F.D./Gilgen, A.R. (Hg.): Chaos theory in psychology (S. 139-144). Westport.

GlanZManN, P. (1985): Zusammenhänge zwischen Angstneigung und Zustandsangst in unterschiedlichen Streß-Situationen. In: Zeitschrift für Differentielle und Diagnostische Psychologie 6, S. 161-173.

Grasso, A.T. (2000): The relationship of competitive state anxiety and athletic performance in high school basketball players. Eugene.

Guastello, S.J. (2002): Structural Equations Technique for Testing Hypotheses in Nonlinear Dynamics. Internet: http://www.societyforchaostheory.org/structuraleq.pps $03 / 2003$.

Guastello, S.J. (2001): Nonlinear Dynamics in Psychology. In: Discrete Dynamics in Nature and Society 6, S. 11-29.

GuAstello, S.J. (1995): Chaos, catastrophe, and human affairs: Applictions of nonlinear dynamics to work, organizations, and social evolution. Mahwah.

HaAse, H. (1991): Die „Catastrophe-Theory” als Modell-Methode in der Sportpsychologie. In: SINGER, R.: Sportpsychologische Forschungsmethodik: Grundlagen, Probleme, Aspekte (S. 162-168). Köln.

Hackfort, D./Schwenkmezger, P. (1989): Measuring Anxiety in Sports: Perspectives and Problems. In: HACKFORT, D./SPIELBERGER, C.D. (Hg.): Anxiety in Sports: An International Perspective (S. 55-74). Washington.

Hackfort, D./Schwenkmezger, P. (1985): Angst und Angstkontrolle im Sport: Sportrelevante Ansätze und Ergebnisse theoretischer und empirischer Angstforschung. Köln. 
HACKForT, D./Spielberger, C.D. (Hg.) (1989): Anxiety in Sports: An international perspective. Washington.

HÄCKER, H. (1983): $\quad$ Aufmerksamkeit und Leistung.

In: Janssen, J.P./HAhN, E. (Hg.): Aktivierung, Motivation, Handlung und Coaching im Sport (S. 37-58). Schorndorf.

HAmilton, J.S. (1997): 8-way Seminar Notes: Brainlocking. Internet: http://www.geocities.com/Pipeline/2246/seminar2.html. 09/2003.

Hanges, P.J./Lord, R.G./Godfrey, E.G./RAver, J.L. (2002): Modeling Nonlinear Relationships: Neural Networks and Catastrophe Analysis.

In: RogelberG, S.G. (Hg.): Handbook of Research Methods in Industrial and Organizational Psychology (S. 431-455). Malden.

HANIN, Y.L. (2000): $\quad$ Individual Zones of Optimal Functioning (IZOF) Model: Emotion-Performance Relationship in Sport. In:

HANIN, Y.L. (Hg.): Emotions in Sport (S. 65-89). Champaign.

HARDY, L. (1997): $\quad$ The Coleman Roberts Griffith Address: Three Myths about Applied Consultancy Work.

In: Journal of Applied Sport Psychology 9, S. 277-294.

HARDY, L. (1996a): $\quad$ A test of catastrophe models of anxiety and sports performance against multidimensional anxiety theory models using the method of dynamic differences.

In: Anxiety, Stress and Coping 9, S. 69-86.

HARDY, L. (1996b): Testing the predictions of the cusp catastrophe model of anxiety and performance. In: The Sport Psychologist 10, S. 140-156.

HARDY, L. (1990): $\quad$ A catastrophe model of performance in sport.

In: Jones, J.G./HARDY, L. (Hg.): Stress and performance in sport (S. 81-106). Chichester.

HARDY, L./HAGTVET, K.A. (1996): Anxiety and performance: Measurement and modelling issues. In: Anxiety, Stress and Coping 9, S. v-viii. 
HARDY, L./PARFITT, G. (1991): A catastrophe model of anxiety and performance.

In: British Journal of Psychology 82, S. 163-172.

Hardy, L./PARfitt, G./Pates, J. (1994): Performance catastrophes in sport: A test of the hysteresis hypothesis. In: Journal of Sports Sciences 12, S. $327-$ 334 (zit. nach HARDY 1996b).

HELLER, K. (2002): $\quad$ Fallschirmspringen. München.

Hindel, C./Krohne, H.W. (1988): Beziehungen von Ängstlichkeit, Angst und Streßbewältigung zum Erfolg bei Leistungssportlern. In: Zeitschrift für Differentielle und Diagnostische Psychologie 9, S. 39-50.

HoEnLE, R. (1996): $\quad$ Deutsche Rekorde im Formationsspringen: Eine Chronik.

In: Fallschirmsportmagazin Heft 1/1996, S. 24-27.

Hollmann, W./HetTingeR, T. (2000): Sportmedizin. Stuttgart.

Humara, M. (1999): The Relationship Between Anxiety and Performance: A Cognitive-Behavioral Perspective. In: Athletic Insight. The Online Journal of Sport Psychology 1 (2).

Internet: http://www.athleticinsight.com. 08/2003.

IDZIKOWSKI, C./BADDELEY, A. (1987): Fear and performance in novice parachutists.

In: Ergonomics 30, S. 1463-1474.

Imlay，G.J./CARDA，R.D./Stanbrough，M.E./Dreiling，A.M./O’ConnOR，P.J. (1995): Anxiety and Athletic Performance: A Test of Zone of Optimal Function Theory.

In: Journal of Sport Psychology 26, S. 295-306.

JEFFrIES, J. (1998a): Basic Exit Techniques. Internet: $\underline{\text { http://www.mariosantos.com/ }}$ docs/basic exit techniques.htm. 03/2003.

JEFFRIES, J. (1998b): Random Work. Internet: http://www.mariosantos.com/docs/ random_work.htm. 03/2003. 
JEFFRIES, J. (1997):

Performing Your Best in Competition. Internet: http://www.geocities.com/Pipeline/2246/seminar3.html. 09/2003.

Jerome, G.J./WiLliams, J.M. (2000): Intensity and Interpretation of Competitive State Anxiety: Relationship to Performance and Repressive Coping. In: Journal of Applied Sport Psychology 12, S. 236-250.

JONES, G. (1995): $\quad$ More than just a game: Research developments and issues in competitive anxiety in sport.

In: British Journal of Psychology 86, S. 449-478.

Karge, K. (1994): Angst und Angstverarbeitung. Eine Untersuchung an Fallschirmspringern. Hannover.

KerR, J.H. (1997): $\quad$ Motivation and Emotion in Sport. Hove.

Kleine, D./SCHWARZER, R. (1991): Angst und sportliche Leistung - eine Meta-Analyse. In: Sportwissenschaft 21, S. 9-28.

KIRCHNER, G./StÖBer, K. (1994): Ordnung in der Vielfalt - taxonomische Ansätze und Anforderungsprofile. In: Hirtz, P./Kirchner, G./PÖHLMAnN, R. (Hg.): Sportmotorik: Grundlagen, Anwendungen und Grenzgebiete (S. 335-383). Kassel.

KJørmo, O./Halvari, H. (2002): Two Ways Related to Perfomance in Elite Sport: The Path of Self-Confidence and Competitive Anxiety and the Path of Group Cohesion and Goal-Clarity. In: Perceptual and Motor Skills 94, S. 950-966.

KopP, K.H./Huber, G./KeUL, J. (1978): Veränderungen von Herzfrequenz und Stoffwechselparameter im Blut beim Fallschirmspringen. In: Deutsche Zeitschrift für Sportmedizin 2, S. 44-49.

Kragh, J.F./TAYLOR, D.C. (1996): Parachuting injuries: A medical analysis of an airborne operation. In: Military Medicine 161, S. 67-69. 
Krane, V./Williams, J. (1994): Cognitive anxiety, somatic anxiety and confidence in track and field athletes: The impact of gender, competitive level and task characteristics.

In: Journal of Sport Psychology 25, S. 203-217.

Krane, V./Williams, J. (1987): Performance and somatic anxiety, cognitive anxiety and confidence changes prior to competition.

In: Journal of Sport Behavior 10, S. 47-56.

KREMPEL, R. (1985): Zur Bewegungsbeurteilung und Fehleranalyse bei leichtathletischen Sprungbewegungen. In: HAHN, E./SCHOCK, K.K. (Hg.): Beiträge zu Kognition und Motorik. Bericht über die Tagung der Arbeitsgemeinschaft für Sportpsychologie (S. 149155). Köln (zit. nach ZIMMERMANN 2001).

KubZANSKY, L.D./STEWART, A.J. (1999): At the intersection of anxiety, gender, and performance.

In: Journal of Social and Clinical Psychology 18, S. 76-97.

Lange, R./Houran, J. (2000): Modelling Maher's Attribution Theory of Delusions as a Cusp Catastrophe. In: Nonlinear Dynamics, Psychology, and Life Sciences 4, S. 235-254.

Lange, R./Oliva, T.A./McDade, S.R. (2001): An Algorithm for Estimating Multivariate Catastrophe Models: GEMCAT II. In: Studies in Nonlinear Dynamics and Econometrics 4, S. 137-168.

Laux, L./Glanzmann, P. (1996): Angst und Ängstlichkeit. In: Amelang, M.: Temperaments- und Persönlichkeitsunterschiede (S. 107-151). Göttingen.

LauX, L./Glanzmann, P./Schaffner, P./Spielberger, C.D. (1981): Das State-TraitAngstinventar. Theoretische Grundlagen und Handanweisung. Weinheim.

LAZARUS, R.S. (2000): How Emotions Influence Performance in Competitive Sports. In: The Sport Psychologist 14, S. 229-252. 
LAZARUS, R.S./FolKMAN, S. (1984): Stress, appraisal, and coping. New York (zit. nach ANSHEL et al. 1997).

LAZARUs-MAinKa, G./SiEBENEICK, S. (2000): Angst und Ängstlichkeit. Göttingen.

LePsien, U./Thorwesten, G./LinNENBECKeR, S./JEROsCH, J. (1998): Verletzungsinzidenz beim Fallschirmsprung.

In: Sportverletzung - Sportschaden 12, S. 71-73.

LEvine, S. (1978): Cortisol Changes Following Repeated Experiences with Parachute Training.

In: Ursin, H./BAAde, E./Levine, S. (Hg.): Psychobiology of Stress. A Study of Coping Men (S. 51-56). New York.

Levine, S./Weinberg, J./Ursin, H. (1978): Definition of the Coping Process and Statement of the Problem.

In: Ursin, H./BaAde, E./Levine, S. (Hg.): Psychobiology of Stress. A Study of Coping Men (S. 3-21). New York.

Lewis-LanZA, M. (2000): Nonlinear Dynamics: Chaos and Catastrophe Theory. In: Journal of Nursing Care Quality 15, S. 55-65.

LOW, R./MCGRATH, J.E. (1971): Stress, arousal, and performance: Some findings calling for a new theory. Project Report AF, S. 1161-1167 (zit. nach HÄCKER 1983).

Machac, M./Machacova, H. (1994): Psychoregulation for peak performance. In: SeILER, R./HACKFORT, D./Allmer, H./Nitsch, J.H. (Hg.): Bewegung und Sport - Psychologische Grundlagen und Wirkungen. Bd. 3: Psychologisches Training (S. 69-78). Sankt Agustin.

Mack, M.G./Huddleston, S./Dutler, K.E./Mintah, J.K. (2000): Chaos Theory: A New Science for Sport Behavior? In: Athletic Insight 2, Issue 2. Internet: http://www.athleticinsight.com.

Malmstrom, K. (1965): Heart rate measurement and the correlation of indices of arousal. In: Psychosomatic Medicine 25, S. 546-556. 
Malone, J. (2001): $\quad$ Team Sebastian XL: Mental and Physical Preparation. In: Skydive: The Mag Heft 06/2001, S. 23-24.

DE MAREeS, H. (1992): $\quad$ Sportphysiologie. Köln.

Martens, R./Vealey, R.S./Burton, D. (1990): Competitive anxiety in sport. Champaign.

MAUSHAKE, M. (1987): Das Fallschirmspringen - eine Untersuchung dieser Abenteuersportart auf ihren sportpädagogischen Wert. Hamburg.

MCNally, I.M. (2002): Contrasting Concepts of Competitive State-Anxiety in Sport: Multidimensional Anxiety and Catastrophe Theories. In: Athletic Insight. The Online Journal of Sport Psychology 4 (2). Internet: http://www.athleticinsight.com. 08/2003.

MeINEL, K./SCHNABEL, G. (1987): Bewegungslehre - Sportmotorik. Berlin.

Metni, A. (2001): $\quad$ Your Optimum Arousal Level. Internet: http://tunnelcamp.com/coaches_corner/arousal.htm. 11/2001.

MiLls, B.D. (1992): Catastrophe model of anxiety and performance: Application to field hockey. Eugene.

Morgan, W.P./Ellickson, K.A. (1989): Health, Anxiety, and Physical Exercise. In: Hackfort, D./Spielberger, C.D. (Hg.): Anxiety in Sports: An International Perspective (S. 165-182). Washington.

MÜLleR, B. (1989): Die Bedeutung der modernen Trainingswissenschaft für den Fallschirmsport. Teil IV.

In: Fallschirmsportmagazin Heft 11/1989, S. 18-25.

Mullen, R./HARDY, L. (2000): State anxiety and motor performance: Testing the conscious processing hypothesis. In: Journal of Sport Sciences 18, S. 785-799. 
Muthny, F.A./SÜSS, K. (2003): Der Fallschirmsprung: Psychophysiologische Extrembelastung, militärischer Mythos und Faszinosum für Jugendliche. In: Klosinski, G. (Hg.): Grenz- und Extremerfahrungen im interdisziplinären Dialog (S. 239-249). Tübingen.

NorUM, K./Ursin, H. (1978): Fatty Acid Mobilization.

In: Ursin, H./BAade, E./Levine, S. (Hg.): Psychobiology of Stress. A Study of Coping Men (S. 75-81). New York.

Noteboom, J.T./BARnholt, K.R./ENOKA, R.M. (2001): Activation of the arousal response and impairment of performance increase with anxiety and stressor intensity.

In: Journal of Applied Physiology 91, S. 2093-2101.

Ntoumanis, N./BiddLe, S.J.H. (2000): Relationship of Intensity and Direction of Competitive Anxiety With Coping Strategies.

In: The Sport Psychologist 14, S. 360-371.

Oliva, T.A./DESARBo, W.S./DAY, D.L./JEDIDI, K. (1987): GEMCAT: A general multivariate methodology for estimating catastrophe models.

In: Behavioral Science 32, S. 121-137.

OrBACH, I. (1999): The relationship between self-confidence and competitive anxiety in influencing sport performance. In:

Dissertation Abstracts International: Section B: The Sciences \& Engineering 60, S. 874.

OXENDINE, J.B. (1970): $\quad$ Emotional arousal and motor performance. In: Quest 13, 23-32 (zit. nach KLEINE/SCHWARZER 1991).

PAIN, M.D. (1999): The moderating role of self-confidence on the performanceanxiety relationship. Poster auf dem 5ten IOC Weltkongress der Sportwissenschaften in Sydney. Internet:

http://www.ausport.gov.au/fulltext/1999/abs076b.htm. 
PARFITT, C.G./JONES, J.G./HARDY, L. (2000): Multidimensional anxiety and performance.

In: JONES, J.G./HARDY, L. (Hg.): Stress and performance in sport (S. 43-80). Chichester.

Parfitt, G./PAtes, J. (1999): The effects of cognitive and somatic anxiety and selfconfidence on components of performance during competition. In: Journal of Sport Sciences 17, S. 351-356.

Powell, F.M./VERnER, J.P. (1982): Anxiety and performance relationships in first time parachutists. In: Journal of Sport Psychology 4, S. 184-188.

PsCHYREMBEL, W. (1993): Medizinisches Wörterbuch. Berlin.

Raglin, J.S./Hanin, Y.L. (2000): Competitive Anxiety. In: Hanin, Y.L. (Hg.): Emotions in Sport (S. 93-111). Champaign.

Randle, S./Weinberg, R. (1997): Multidimensional Anxiety and Performance: An Exploratory Examination of the Zone of Optimal Functioning Hypothesis. In: The Sport Psychologist 11, S. 160-174.

RAudSEPP, L./KaIS, K. (2002): The Relationship between State Anxiety and Performance in Beach Volleyball Players.

In: Journal of Human Movement Studies 43, S. 403-416.

REID, D.H./Doerr, J.E./Doshier, H.D./Ellertson, D.G. (1971): Heart Rate and Respiration Rate Response to Parachuting: Physiological Studies of Military Parachutists Via FM/FM Telemetry-II.

In: Aerospace Medicine 42, S. 1200-1207.

RICHTER, S.D./SchÜRMEYER, T.H./SCHEDlOWSKI, M./HÄDICKE, A./TEWES, U./SCHMIDT, R.E./WAGNER, T.O.F. (1996): Time Kinetics of the Endocrine Response to Acute Psychological Stress. In: Journal of Clinical Endocrinology and Metabolism 81, S. 1956-1960.

Rost, R. (Hg.) (2001a): $\quad$ Lehrbuch der Sportmedizin. Köln.

Rost, W. (2001b): $\quad$ Emotionen: Elixiere des Lebens. Berlin. 
Roth, G./MEnZEL, R. (1996): Neuronale Grundlagen kognitiver Leistungen.

In: Dudel, J./MENZEL, R./SCHMIDT, R.F.: Neurowissenschaft: Vom Molekül zur Kognition (S. 539-560). Berlin.

Roth, W.T./Breivik, G./Jørgensen, P.E./Hofmann, S. (1996): Activation in novice and expert parachutists while jumping.

In: Psychophysiology 33, S. 63-72.

SAlminen, S./Liukkonen, J./HAnin, Y./Hyvönen, A. (1995): Anxiety and athletic performance of Finnish athletes: Application of the zone of optimal functioning model.

In: Personality and Individual Differences 19, S. 725-730.

SAnNS, W. (2000): Catastrophe Theory with Mathematica: A geometric approach. Osnabrück.

SAUNDERS, P.T. (1986): Katastrophentheorie. Eine Einführung für Naturwissenschaftler. Braunschweig.

SCHÄFER, P. (1998): $\quad$ Fallschirmspringen. Augsburg.

Schedlowski, M./Tewes, U. (1992): Physiological Arousal and Perception of Bodily State During Parachute Jumping. In: Psychophysiology 29, S. 95-103.

SCHIEDEK, S. (2000): Optimal arousal level for Skydiving. In: Australian Parachute Federation's SkyLine Heft 04/2000, S. 10-11.

SCHWARZER, R. (1993): $\quad$ Streß, Angst und Handlungsregulation. Stuttgart.

Shane, W.P./SLINDE, K.E. (1968): Continuous ECG recording during free fall parachuting. In: Aerospace Medicine 6, S. 597-603.

Sharma, V.M./Sridharan, K./Selvamurthy, W./MukherJeE, A.K. (1994): Personality traits and performance of military parachutist trainees. In: Ergonomics 37, S. 1145-1155.

SIEVERS, W. (1995): $\quad$ Elemente der Statistik. Göttingen. 
Snell, J.C./Cangemi, J.P./Kowalski，C.J./Noble，C./Payne，K.E. (1999): The New Science: Chaos Theory, Catastrophic Theory, And Topological Theory. In: Psychology 36, S. 24-29.

SöRENSEN, M. (1994): $\quad$ Einführung in die Angstpsychologie. Weinheim.

SONSTROEM, R.J./BERNARDO, P. (1982): Intraindividual pregame state anxiety and basketball performance: A re-examination of the inverted-U curve. In: Journal of Sport Psychology 4, S. 235-245 (zit. nach ZIMMERMANN 2001).

STAFFORD, T. (1996): Mental Preparation and Visualisation. In: Sport Parachutist Heft 06/1996, S. 33-35.

SteinBACH, M. (1968): $\quad$ Über den Vorstartzustand. In: Praxis der Leibesübungen 9, S. 203-204.

TAYLOR, J. (1987): $\quad$ Predicting athletic performance with self-confidence and somatic and cognitive anxiety as a function of motor and physiological requirements in six sports.

In: Journal of personality 55, S. 139-153.

Turner, P.E./Raglin, J.S. (1991): Anxiety and performance in track and field athletes: A comparison of ZOFR and inverted-U hypothesis.

In: Medical Science in Sport and Exercise 23, S. 119 (zit. nach HUMARA 1999).

UlBRICHT, K. (1994): $\quad$ Über Katastrophentheorie und Anwendungen. Neubiberg.

VALlERAND, R.J./Blanchard, C.M. (2000): The Study of Emotion in Sport and Exercise. In: HANIN, Y.L. (Hg.): Emotions in Sport (S. 3-37). Champaign.

Vogel, H. (1997): $\quad$ Gerthsen Physik. Berlin.

Weder, G. (2002): Optimale Handlung - am Beispiel hoher Geschwindigkeit. Zürich. 
Weinberg, R. (1989): Anxiety, Arousal, and Motor Performance: Theory, Research, and Applications.

In: HaCKForT, D./SpielberGer, C.D. (Hg.): Anxiety in Sports: An International Perspective (S. 95-115). Washington.

WILLIAMS, J.M./HARRIS, D. (1998): Relaxation and energizing techniques for regulation of arousal.

In: Williams, J.M. (Hg.): Applied sport psychology: Personal growth to peak performance (S. 219-233). Mayfield

(zit. nach COHEN et al. 2003).

WiLlimCZIK, K. (Hg.) (1997): Statistik im Sport. Hamburg.

ZEEMAN, E.C. (1995): $\quad$ Catastrophe theory: Conference by E.C. Zeeman. San Antonio. Internet: http://www.math.utsa.edu/ecz/c.html. 09/2003.

ZEEMAN, E.C. (1977): $\quad$ Catastrophe theory. Selected papers 1972-1977. Reading.

ZIMMERMANN, K.W. (2001): Sportpsychologie - eine praxisfeldorientierte Einführung. Internet: http://www.uni-kassel.de/fb3/psych/pers/zimmermann/ SPOPS-tot-2001.pdf. 10/2001.

\section{Weitere Quellen:}

CoBB, L.:

An Introduction to Cusp Surface Analysis. Internet: http://www.aetheling.com/models/cusp/Intro.htm. 09/2003.

DUJARDIN, L.: Catastrophe teacher. Introduction for experimentalists. Internet: http://perso.wanadoo.fr/l.d.v.dujardin/ct/eng_index.html. 09/2003.

Oliva, T.A.: $\quad$ Catastrophe Theory Estimation Program GEMCAT II. Internet: http://www.sbm.temple.edu/ oliva/cat-theroy.htm. 09/2003.

Wagner, T./WAgner, T.: Omniskore: Competition Formation Skydiving Headquarters. Internet: http://www.omniskore.com. 09/2003. 


\section{Anhang}

- Anhang A: Verwendete Fragebögen (Seite 163)

- Anhang B: Ausgabe des Programms GEMCAT II: Angepasste Spitzenkatastrophenflächen für die verschiedenen Untersuchungsgruppen (Seite 175)

- Anhang C: Ausgabe des Programms GEMCAT II: Angepasste Schmetterlingskatastrophenflächen für die Untersuchungsgruppen unterschiedlicher Eigenschaftsangst (Seite 184)

- Anhang D: Freifallformationsprogramm 4er-Disziplin (Seite 187)

- Anhang E: Freifallformationsprogramm 8er-Disziplin (Seite 191) 


\section{Verwendete Fragebögen}

\section{Information zu Deinen Fragebögen}

Die psychischen Effekte von Hochleistungssport können sehr stark und sehr unterschiedlich unter den beobachteten Athleten und Sportlern sein. Der Fragebogen, den Du bei der Untersuchung ausfüllen wirst, misst, wie Deine Gefühle im Wettbewerb zu verschiedenen Zeitpunkten des Sprunges sind. Bitte fülle den Bogen so ehrlich wie möglich aus.

Manchmal meinen Sportler, dass es besser wäre, Nervosität, Angst oder Sorgen, die sie vor einem Wettbewerb empfinden, nicht zuzugeben. Schließlich könnten diese Gefühle "unerwünscht" sein.

Tatsächlich sind diese Gefühle aber recht häufig - für eine bessere Erforschung der Zusammenhänge von Angst, Stress und Leistung solltest Du Deine Empfindungen mit mir teilen. Wenn Dir der Wettbewerb Sorgen bereitet, Du Schmetterlinge im Bauch hast oder andere Dinge verspürst, die bei Dir ein Anzeichen von Angst oder Erregung sind, bitte benenne diese Gefühle an der entsprechenden Stelle des Fragebogens.

Analog gilt dies natürlich auch, falls Du Dich ruhig und entspannt fühlst, markiere dann auch bitte diese Gefühle auf dem Fragebogen.

Deine Angaben werden streng vertraulich behandelt. Zusätzlich interessiere ich mich nicht für einzelne Antworten. Der Bogen wird nur nach Antwortgruppen ausgewertet. Vielen Dank.

\section{The questionnaires}

The effects of highly competitive sports can be powerful and very different among athletes. The inventory you are about to complete measures how you feel about this competition at the moment you are responding. Please complete the inventory as honestly as you can.

Sometimes athletes feel they should not admit to any nervousness, anxiety or worry they experience before competition because this is undesirable.

Actually, these feelings are quite common, and to help us understand them I want you to share your feelings with me candidly. If you are worried about the competition or have butterflies or other feelings that you know are signs of anxiety, please indicate these feelings accurately on the inventory.

Equally, if you feel calm and relaxed, indicate those feelings as accurately as you can.

Your answers will not be shared with anyone. In addition to this, I won't be evaluating your individual answers. I will be looking only at group responses. 
Name:

Team:

Datum:

Sprungart: Training / Wettkampf Runde Nr.:

Repeater? Ja/ Nein

\section{Bitte beantworte diese Fragen direkt nach dem Sprung.}

Unten sind einige Aussagen von Personen, die beschreiben, wie sie sich im Wettkampf fühlen. Lies die einzelnen Aussagen und markiere den Buchstaben, der am besten Deine Gefühle in drei Momenten vor und während Deinem letzten Sprung beschreibt.

Es gibt bei diesem Fragebogen keine richtigen oder falschen Antworten. Überlege bitte nicht allzu lange, was Du bei einer Aussage ankreuzen sollst. Denke daran, daß Du den Buchstaben ankreuzt, welcher Deine Gefühle zu den drei Zeiten bei deinem letzten Sprung beschreibt.

\begin{tabular}{|c|c|c|c|c|c|c|}
\hline & & Zeit & $\begin{array}{l}\text { Überhaupt } \\
\text { Nicht }\end{array}$ & $\begin{array}{c}\text { Ein } \\
\text { Wenig }\end{array}$ & Ziemlich & Sehr \\
\hline \multirow{3}{*}{\multicolumn{2}{|c|}{$\begin{array}{l}\text { 1. Ich mache mir Sorgen über diesen } \\
\text { Sprung. }\end{array}$}} & Start & $\bar{A}$ & $\mathrm{~B}$ & $\mathrm{C}$ & $\mathrm{D}$ \\
\hline & & $1 \mathrm{~min}$ vor Exit & $\mathrm{A}$ & B & $\mathrm{C}$ & $\mathrm{D}$ \\
\hline & & Im Freifall & A & B & $\mathrm{C}$ & $\mathrm{D}$ \\
\hline \multirow{3}{*}{\multicolumn{2}{|c|}{ 2. Ich bin nervös. }} & Start & A & B & $\mathrm{C}$ & D \\
\hline & & $1 \mathrm{~min}$ vor Exit & A & B & $\mathrm{C}$ & $\mathrm{D}$ \\
\hline & & Im Freifall & A & B & $\mathrm{C}$ & $\mathrm{D}$ \\
\hline \multirow{3}{*}{\multicolumn{2}{|c|}{$\begin{array}{l}\text { 3. Ich fühle mich völlig entspannt und } \\
\text { locker in dieser Situation. }\end{array}$}} & Start & $\mathrm{A}$ & B & C & $\mathrm{D}$ \\
\hline & & $1 \mathrm{~min}$ vor Exit & A & B & $\mathrm{C}$ & $\mathrm{D}$ \\
\hline & & Im Freifall & A & B & $\mathrm{C}$ & $\mathrm{D}$ \\
\hline \multirow{3}{*}{\multicolumn{2}{|c|}{ 4. Ich habe Selbstzweifel. }} & Start & $\mathrm{A}$ & B & C & $\mathrm{D}$ \\
\hline & & 1 min vor Exit & A & B & $\mathrm{C}$ & $\mathrm{D}$ \\
\hline & & Im Freifall & A & B & C & $\mathrm{D}$ \\
\hline \multirow{3}{*}{\multicolumn{2}{|c|}{ 5. Ich bekomme „, das große Flattern“. }} & Start & A & B & $\mathrm{C}$ & $\mathrm{D}$ \\
\hline & & $1 \mathrm{~min}$ vor Exit & $\mathrm{A}$ & B & $\mathrm{C}$ & $\mathrm{D}$ \\
\hline & & Im Freifall & A & $\mathrm{B}$ & C & $\mathrm{D}$ \\
\hline \multirow{3}{*}{\multicolumn{2}{|c|}{ 6. Ich fühle mich als Herr der Lage. }} & Start & A & B & $\mathrm{C}$ & $\mathrm{D}$ \\
\hline & & $1 \mathrm{~min}$ vor Exit & $\mathrm{A}$ & $\mathrm{B}$ & $\mathrm{C}$ & $\mathrm{D}$ \\
\hline & & Im Freifall & A & B & $\mathrm{C}$ & $\mathrm{D}$ \\
\hline \multirow{3}{*}{\multicolumn{2}{|c|}{$\begin{array}{l}\text { 7. Ich habe die Sorge, in diesem } \\
\text { Sprung nicht so gut zu sein, wie ich } \\
\text { könnte. }\end{array}$}} & Start & A & B & C & $\mathrm{D}$ \\
\hline & & $1 \mathrm{~min}$ vor Exit & A & $\mathrm{B}$ & $\mathrm{C}$ & $\mathrm{D}$ \\
\hline & & Im Freifall & A & B & $\mathrm{C}$ & $\mathrm{D}$ \\
\hline \multirow{3}{*}{\multicolumn{2}{|c|}{ 8. Mein Körper ist angespannt. }} & Start & A & B & C & $\mathrm{D}$ \\
\hline & & 1min vor Exit & $\mathrm{A}$ & B & $\mathrm{C}$ & $\mathrm{D}$ \\
\hline & & Im Freifall & A & $B$ & C & $\mathrm{D}$ \\
\hline \multirow{3}{*}{\multicolumn{2}{|c|}{ 9. Ich bin selbstsicher. }} & Start & $\mathrm{A}$ & B & C & $\mathrm{D}$ \\
\hline & & 1min vor Exit & $\mathrm{A}$ & B & $\mathrm{C}$ & $\mathrm{D}$ \\
\hline & & Im Freifall & A & B & $\mathrm{C}$ & $\mathrm{D}$ \\
\hline \multirow{3}{*}{\multicolumn{2}{|c|}{ 10. Ich habe Angst, zu verlieren. }} & Start & A & $\mathrm{B}$ & $\mathrm{C}$ & $\mathrm{D}$ \\
\hline & & $1 \mathrm{~min}$ vor Exit & A & B & $\mathrm{C}$ & $\mathrm{D}$ \\
\hline & & Im Freifall & A & $\mathrm{B}$ & $\mathrm{C}$ & $\mathrm{D}$ \\
\hline \multirow{3}{*}{\multicolumn{2}{|c|}{$\begin{array}{l}\text { 11. Ich habe ein flaues Gefühl in der } \\
\text { Magengegend. }\end{array}$}} & Start & A & B & $\mathrm{C}$ & $\mathrm{D}$ \\
\hline & & $1 \mathrm{~min}$ vor Exit & $\mathrm{A}$ & B & $\mathrm{C}$ & $\mathrm{D}$ \\
\hline & & Im Freifall & A & $\mathrm{B}$ & $\mathrm{C}$ & $\mathrm{D}$ \\
\hline \multirow{3}{*}{\multicolumn{2}{|c|}{ 12. Ich fühle mich sicher. }} & Start & A & B & $\mathrm{C}$ & $\mathrm{D}$ \\
\hline & & 1 min vor Exit & $\mathrm{A}$ & B & $\mathrm{C}$ & $\mathrm{D}$ \\
\hline & & Im Freifall & A & B & $\mathrm{C}$ & $\mathrm{D}$ \\
\hline \multirow{3}{*}{\multicolumn{2}{|c|}{$\begin{array}{l}\text { 13. Ich mache mir Sorgen, unter dem } \\
\text { Wettbewerbsdruck zu brainlocken. }\end{array}$}} & Start & A & B & C & $\mathrm{D}$ \\
\hline & & $1 \mathrm{~min}$ vor Exit & A & B & $\mathrm{C}$ & $\mathrm{D}$ \\
\hline & & Im Freifall & $\mathrm{A}$ & B & $\mathrm{C}$ & $\mathrm{D}$ \\
\hline
\end{tabular}

..nächste Seite... 


\begin{tabular}{|c|c|c|c|c|c|c|}
\hline & & Zeit & $\begin{array}{l}\text { Überhaupt } \\
\text { Nicht }\end{array}$ & $\begin{array}{c}\text { Ein } \\
\text { Wenig }\end{array}$ & Ziemlich & Sehr \\
\hline \multirow{3}{*}{\multicolumn{2}{|c|}{$\begin{array}{l}\text { 14. Mein Körper fühlt sich } \\
\text { entspannt an. }\end{array}$}} & Start & A & B & $\mathrm{C}$ & $\mathrm{D}$ \\
\hline & & 1 min vor Exit & A & B & $\mathrm{C}$ & $\mathrm{D}$ \\
\hline & & Im Freifall & A & B & C & $\mathrm{D}$ \\
\hline \multirow{3}{*}{\multicolumn{2}{|c|}{$\begin{array}{l}\text { 15. Ich bin mir sicher, dass ich die } \\
\text { Herausforderung bewältigen } \\
\text { kann. }\end{array}$}} & Start & A & B & $\mathrm{C}$ & $\mathrm{D}$ \\
\hline & & 1 min vor Exit & A & B & $\mathrm{C}$ & $\mathrm{D}$ \\
\hline & & ImFreifall & A & B & C & $\mathrm{D}$ \\
\hline \multirow{3}{*}{\multicolumn{2}{|c|}{$\begin{array}{l}\text { 16. Ich habe Angst, ich könnte } \\
\text { einen schlechten Sprung } \\
\text { zeigen. }\end{array}$}} & Start & $\mathrm{A}$ & B & $\mathrm{C}$ & $\mathrm{D}$ \\
\hline & & $1 \mathrm{~min}$ vor Exit & A & B & $\mathrm{C}$ & $\mathrm{D}$ \\
\hline & & Im Freifall & $A$ & B & $\mathrm{C}$ & $\mathrm{D}$ \\
\hline \multirow{3}{*}{\multicolumn{2}{|c|}{ 17. Mein Herz rast. }} & Start & A & B & C & $\mathrm{D}$ \\
\hline & & $1 \mathrm{~min}$ vor Exit & A & B & $\mathrm{C}$ & $\mathrm{D}$ \\
\hline & & Im Freifall & A & B & $\mathrm{C}$ & $\mathrm{D}$ \\
\hline \multirow{3}{*}{\multicolumn{2}{|c|}{$\begin{array}{l}\text { 18. Ich bin mir sicher, dass ich eine } \\
\text { gute Leistung bringe. }\end{array}$}} & Start & A & B & $\mathrm{C}$ & $\mathrm{D}$ \\
\hline & & $1 \mathrm{~min}$ vor Exit & A & B & $\mathrm{C}$ & $\mathrm{D}$ \\
\hline & & Im Freifall & A & $\mathrm{B}$ & $\mathrm{C}$ & $\mathrm{D}$ \\
\hline \multirow{3}{*}{\multicolumn{2}{|c|}{$\begin{array}{l}\text { 19. Ich mache mir Gedanken, ob } \\
\text { ich meine Ziele erreichen kann. }\end{array}$}} & Start & A & B & C & $\mathrm{D}$ \\
\hline & & $1 \mathrm{~min}$ vor Exit & A & B & $\mathrm{C}$ & $\mathrm{D}$ \\
\hline & & Im Freifall & A & B & $\mathrm{C}$ & $\mathrm{D}$ \\
\hline \multirow{3}{*}{\multicolumn{2}{|c|}{$\begin{array}{l}\text { 20. Mein Herz rutscht mir in die } \\
\text { Hose. }\end{array}$}} & Start & A & B & $\mathrm{C}$ & D \\
\hline & & 1 min vor Exit & A & B & $\mathrm{C}$ & $\mathrm{D}$ \\
\hline & & Im Freifall & A & B & C & $\mathrm{D}$ \\
\hline \multirow{3}{*}{\multicolumn{2}{|c|}{ 21. Mental bin ich entspannt. }} & Start & A & B & C & D \\
\hline & & 1 min vor Exit & $\mathrm{A}$ & B & $\mathrm{C}$ & $\mathrm{D}$ \\
\hline & & Im Freifall & $A$ & B & C & $\mathrm{D}$ \\
\hline \multirow{3}{*}{\multicolumn{2}{|c|}{$\begin{array}{l}\text { 22. Ich habe Angst, dass andere } \\
\text { von meiner Leistung enttäuscht } \\
\text { sind. }\end{array}$}} & Start & A & B & C & D \\
\hline & & 1 min vor Exit & A & B & $\mathrm{C}$ & $\mathrm{D}$ \\
\hline & & Im Freifall & A & B & $\mathrm{C}$ & $\mathrm{D}$ \\
\hline \multirow{3}{*}{\multicolumn{2}{|c|}{ 23. Meine Hände sind feucht. }} & Start & A & B & C & D \\
\hline & & $1 \mathrm{~min}$ vor Exit & A & B & $\mathrm{C}$ & $\mathrm{D}$ \\
\hline & & Im Freifall & A & B & $\mathrm{C}$ & $\mathrm{D}$ \\
\hline \multirow{3}{*}{\multicolumn{2}{|c|}{$\begin{array}{l}\text { 24. Ich bin zuversichtlich, da ich } \\
\text { mir das Erreichen meiner Ziele } \\
\text { bildlich vorstelle. }\end{array}$}} & Start & A & B & $\mathrm{C}$ & $\mathrm{D}$ \\
\hline & & $1 \mathrm{~min}$ vor Exit & A & B & $\mathrm{C}$ & $\mathrm{D}$ \\
\hline & & Im Freifall & A & B & $\mathrm{C}$ & $\mathrm{D}$ \\
\hline \multirow[t]{3}{*}{25.} & Ich habe die Sorge, mich nicht & Start & A & B & $\mathrm{C}$ & $\mathrm{D}$ \\
\hline & genügend konzentrieren $\mathrm{zu}$ & $1 \mathrm{~min}$ vor Exit & A & B & $\mathrm{C}$ & $\mathrm{D}$ \\
\hline & können. & Im Freifall & A & B & $\mathrm{C}$ & $\mathrm{D}$ \\
\hline \multirow{3}{*}{\multicolumn{2}{|c|}{$\begin{array}{l}\text { 26. Mein Körper } \\
\text { verkrampft an. }\end{array}$}} & Start & A & B & $\mathrm{C}$ & $\mathrm{D}$ \\
\hline & & $1 \mathrm{~min}$ vor Exit & A & B & $\mathrm{C}$ & $\mathrm{D}$ \\
\hline & & Im Freifall & A & B & $\mathrm{C}$ & $\mathrm{D}$ \\
\hline \multirow{3}{*}{\multicolumn{2}{|c|}{$\begin{array}{l}\text { 27. Ich bin mir sicher, daß ich dem } \\
\text { Druck standhalten kann. }\end{array}$}} & Start & A & B & $\mathrm{C}$ & $\mathrm{D}$ \\
\hline & & $1 \mathrm{~min}$ vor Exit & A & B & $\mathrm{C}$ & $\mathrm{D}$ \\
\hline & & Im Freifall & A & B & $\mathrm{C}$ & $\mathrm{D}$ \\
\hline
\end{tabular}

Bitte benote Deine persönliche Leistung bei dem Sprung auf einer Skala von

$0 \%$ bis $100 \%$ (dabei ist $0 \%$ sehr schlecht und $100 \%$ sehr gut): $\%$

Beantworte die nächste Seite (allgemeiner Fragebogen) wann immer Du Zeit hast. 
Name:_ Team:_ Datum:

\section{Bitte gib mir einige allgemeine Informationen über Dich.}

Raucher: Ja / Nein Geschlecht: m / w Alter:___ Größe:____ Gewicht:

Anzahl der Sprünge:

Freifallzeit:

Jahre im Sport:

davon FS Sprünge:

Windtunnel-Zeit:

\section{Exit-Position:}

Bitte beschreibe kurz Deine persönliche Routine im Steigflug als Vorbereitung für den Sprung:

Weitere Kommentare:

Ich möchte gerne eine Kopie meiner Testresultate via E-Mail oder Fax: Ja / Nein

Wenn ja: E-Mail: Fax:

\section{Vielen Dank für Deine Mitarbeit.}

Nur vom Auswerter auszufüllen:

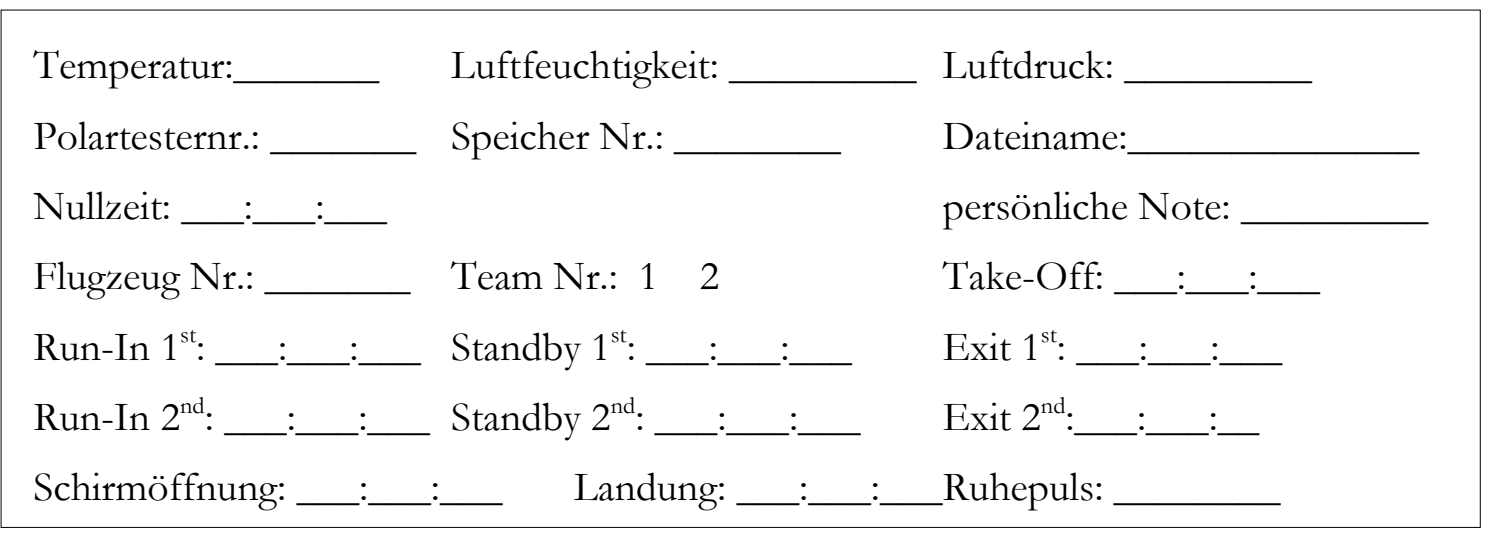


Name:

Team:

Datum:

\section{Bitte beantworte diese Fragen, wann immer Du Zeit hast.}

Im folgenden Fragebogen findest Du eine Reihe von Feststellungen, mit denen man sich selbst beschreiben kann. Bitte lies jede Feststellung durch und wähle aus den vier Antworten diejenige aus, die angibt, wie Du Dich im allgemeinen fühlst. Kreuze bitte bei jeder Feststellung den Buchstaben bei der von Dir gewählten Antwort an.

Es gibt keine richtigen oder falschen Antworten. Überlege bitte nicht lange und denke daran, diejenige Antwort auszuwählen, die am besten beschreibt, wie Du Dich im allgemeinen fühlst.

\begin{tabular}{|c|c|c|c|c|}
\hline & $\begin{array}{l}\text { Fast } \\
\text { Nie }\end{array}$ & Manchmal & Oft & $\begin{array}{c}\text { Fast } \\
\text { Immer }\end{array}$ \\
\hline 1. Ich bin vergnügt. & $\mathrm{A}$ & $\mathrm{B}$ & $\mathrm{C}$ & $\mathrm{D}$ \\
\hline 2. Ich werde schnell müde. & A & $\mathrm{B}$ & C & $\mathrm{D}$ \\
\hline 3. Mir ist zum Weinen zumute. & A & B & $\mathrm{C}$ & D \\
\hline $\begin{array}{l}\text { 4. Ich glaube, mir geht es schlechter als anderen } \\
\text { Leuten. }\end{array}$ & A & $\mathrm{B}$ & C & $\mathrm{D}$ \\
\hline $\begin{array}{l}\text { 5. Ich verpasse günstige Gelegenheiten, weil ich } \\
\text { mich nicht schnell genug entscheiden kann. }\end{array}$ & A & $\mathrm{B}$ & C & $\mathrm{D}$ \\
\hline 6. Ich fühle mich ausgeruht. & A & $\mathrm{B}$ & C & D \\
\hline 7. Ich bin ruhig und gelassen. & A & $\mathrm{B}$ & C & D \\
\hline $\begin{array}{l}\text { 8. Ich glaube, dass mir meine Schwierigkeiten über } \\
\text { den Kopf wachsen. }\end{array}$ & A & $\mathrm{B}$ & C & $\mathrm{D}$ \\
\hline $\begin{array}{l}\text { 9. Ich mache mir zuviel Gedanken über unwichtige } \\
\text { Dinge. }\end{array}$ & A & $\mathrm{B}$ & C & $\mathrm{D}$ \\
\hline 10. Ich bin glücklich. & A & B & $\mathrm{C}$ & D \\
\hline 11. Ich neige dazu, alles schwer zu nehmen. & A & $\mathrm{B}$ & $\mathrm{C}$ & $\mathrm{D}$ \\
\hline 12. Mir fehlt es an Selbstvertrauen. & A & $\mathrm{B}$ & $\mathrm{C}$ & $\mathrm{D}$ \\
\hline 13. Ich fühle mich geborgen. & A & $\mathrm{B}$ & $\mathrm{C}$ & D \\
\hline $\begin{array}{l}\text { 14. Ich mache mir Sorgen über mögliches } \\
\text { Missgeschick. }\end{array}$ & A & $\mathrm{B}$ & $\mathrm{C}$ & $\mathrm{D}$ \\
\hline 15. Ich fühle mich niedergeschlagen. & A & B & C & $\mathrm{D}$ \\
\hline 16. Ich bin zufrieden. & A & $\mathrm{B}$ & C & D \\
\hline $\begin{array}{l}\text { 17. Unwichtige Gedanken gehen mir durch den Kopf } \\
\text { und bedrücken mich. }\end{array}$ & A & $\mathrm{B}$ & C & $\mathrm{D}$ \\
\hline $\begin{array}{l}\text { 18. Enttäuschungen nehme ich so schwer, dass ich } \\
\text { sie nicht vergessen kann. }\end{array}$ & A & $\mathrm{B}$ & C & $\mathrm{D}$ \\
\hline 19. Ich bin ausgeglichen. & A & $\mathrm{B}$ & $\mathrm{C}$ & $\mathrm{D}$ \\
\hline $\begin{array}{l}\text { 20. Ich werde nervös und unruhig, wenn ich an } \\
\text { meine derzeitigen Angelegenheiten denke. }\end{array}$ & $\mathrm{A}$ & $\mathrm{B}$ & C & $\mathrm{D}$ \\
\hline
\end{tabular}




Name:
Jump type (Training/Competition) $\quad$ Team: $\quad$ Date: $\overline{\text { Round.: }} \overline{\text { Repeater? } \mathrm{Yes} / \mathrm{No}}$

\section{Please answer this page right after your skydive.}

A number of statements that athletes have used to describe their feelings before competition are given below. Read each statement and then circle the appropriate letter to the right of the statement to indicate how you felt when you where on the plane ride to your last skydive ${ }^{156}$.

There are no right or wrong answers. Do not spend too much time on any one statement, but choose the answer which describes your feelings during the ride to altitude on your last skydive.

\begin{tabular}{|c|c|c|c|c|}
\hline & Not At All & Somewhat & $\begin{array}{l}\text { Moderatly } \\
\text { So }\end{array}$ & $\begin{array}{l}\text { Very Much } \\
\text { So }\end{array}$ \\
\hline 1. I am concerned about this jump. & $\bar{A}$ & $\mathrm{~B}$ & $\mathrm{C}$ & $\mathrm{D}$ \\
\hline 2. I feel nervous. & A & B & C & $\mathrm{D}$ \\
\hline 3. I feel at ease. & A & B & C & $\mathrm{D}$ \\
\hline 4. I have self-doubts. & A & $\mathrm{B}$ & $\mathrm{C}$ & $\mathrm{D}$ \\
\hline 5. I feel jittery. & A & $\mathrm{B}$ & $\mathrm{C}$ & $\mathrm{D}$ \\
\hline 6. I feel comfortable. & A & $\mathrm{B}$ & $\mathrm{C}$ & $\mathrm{D}$ \\
\hline $\begin{array}{l}\text { 7. I am concerned that I may not do as } \\
\text { well in this competition as I could. }\end{array}$ & A & $\mathrm{B}$ & $\mathrm{C}$ & $\mathrm{D}$ \\
\hline 8. My body feels tense. & A & B & C & $\mathrm{D}$ \\
\hline 9. I feel self-confident. & A & $\mathrm{B}$ & $\mathrm{C}$ & $\mathrm{D}$ \\
\hline 10. I am concerned about losing. & A & $\mathrm{B}$ & $\mathrm{C}$ & $\mathrm{D}$ \\
\hline 11. I feel tense in my stomach. & A & $\mathrm{B}$ & $\mathrm{C}$ & $\mathrm{D}$ \\
\hline 12. I feel secure. & A & $\mathrm{B}$ & $\mathrm{C}$ & $\mathrm{D}$ \\
\hline $\begin{array}{l}\text { 13. I am concerned about brainlocking } \\
\text { under pressure. }\end{array}$ & A & $\mathrm{B}$ & $\mathrm{C}$ & $\mathrm{D}$ \\
\hline 14. My body feels relaxed. & A & $\mathrm{B}$ & $\mathrm{C}$ & $\mathrm{D}$ \\
\hline 15. I'm confident I can meet the challenge. & A & $\mathrm{B}$ & C & $\mathrm{D}$ \\
\hline $\begin{array}{l}\text { 16. I'm concerned about performing } \\
\text { poorly. }\end{array}$ & A & $\mathrm{B}$ & $\mathrm{C}$ & $\mathrm{D}$ \\
\hline 17. My heart is racing. & A & B & C & $\mathrm{D}$ \\
\hline 18. I'm confident about performing well. & A & $\mathrm{B}$ & $\mathrm{C}$ & $\mathrm{D}$ \\
\hline 19. I'm concerned about reaching my goal. & A & $\mathrm{B}$ & $\mathrm{C}$ & $\mathrm{D}$ \\
\hline 20. I feel my stomach sinking. & A & $\mathrm{B}$ & $\mathrm{C}$ & $\mathrm{D}$ \\
\hline 21. I feel mentally relaxed. & A & B & $\mathrm{C}$ & $\mathrm{D}$ \\
\hline $\begin{array}{l}\text { 22. I'm concerned that others will be } \\
\text { disappointed with my performance. }\end{array}$ & A & $\mathrm{B}$ & $\mathrm{C}$ & $\mathrm{D}$ \\
\hline 23. My hands are clammy. & A & $\mathrm{B}$ & $\mathrm{C}$ & $\mathrm{D}$ \\
\hline $\begin{array}{l}\text { 24. I'm confident because I mentally } \\
\text { picture myself reaching my goal. }\end{array}$ & A & $\mathrm{B}$ & $\mathrm{C}$ & $\mathrm{D}$ \\
\hline $\begin{array}{l}\text { 25. I'm concerned I won't be able to } \\
\text { concentrate. }\end{array}$ & A & $\mathrm{B}$ & $\mathrm{C}$ & $\mathrm{D}$ \\
\hline 26. My body feels tight. & A & $\mathrm{B}$ & $\mathrm{C}$ & $\mathrm{D}$ \\
\hline $\begin{array}{l}\text { 27. I'm confident of coming through under } \\
\text { pressure. }\end{array}$ & A & $\mathrm{B}$ & $\mathrm{C}$ & $\mathrm{D}$ \\
\hline
\end{tabular}

Forward to the next page (general questionaire) whenever you have time

${ }^{156}$ Beginnend mit der englischen Fragebogenfassung wird aus Platzgründen im Anhang nur die Kurzfassung des verwendeten CSAI-2 aufgeführt und nicht die lange Version, die die Angstwerte zu drei Zeiten misst. 
Your Name: __ Country:___ Date:

\section{Please give me some general information about you.}

Smoker: Yes / No Sex: Male / Female Age:

Height:

Weight:

Number of jumps:

FS jumps:

Years in sport:

Freefall Time:

Wind tunnel time:

Exit position:

Please describe your team routine on the ride to altitude (shortly):

Comments:

I would like to receive a copy of my personal test results via e-Mail or fax: Yes No

If Yes: E-Mail: Fax:

Thanks a lot for your cooperation.

My use only:

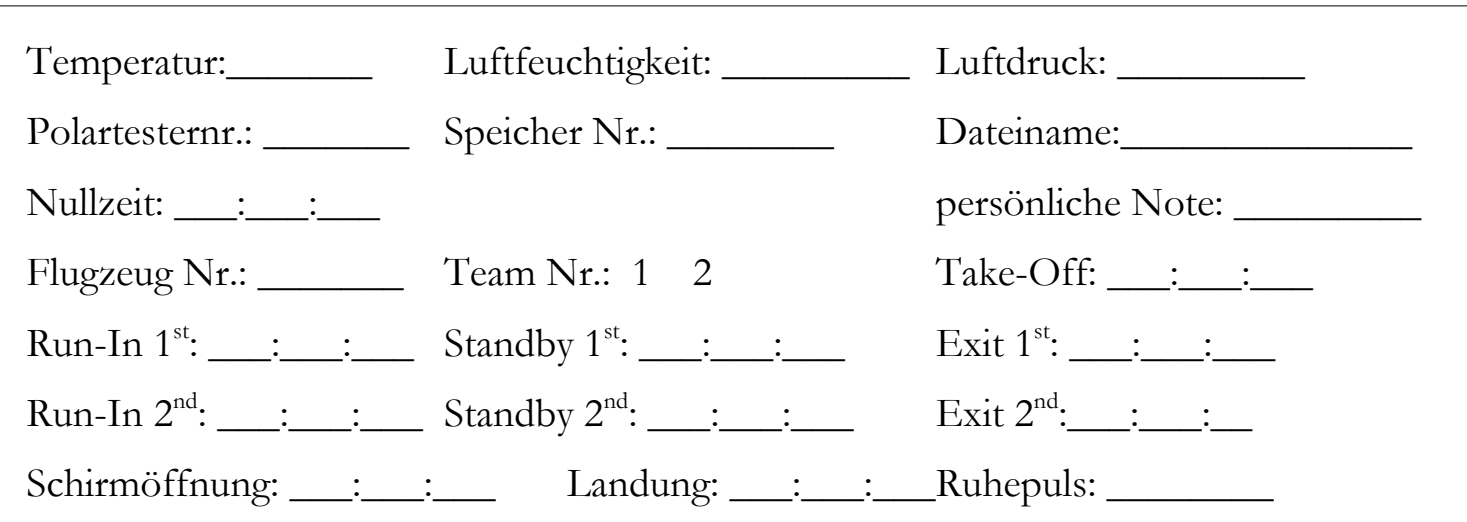


Your Name:

Country:

Date:

\section{Please answer this page whenever you have time.}

A number of statements which people have used to describe themselves are given below. Read each statement and then mark the appropriate letter to the right of the statement to indicate how you generally feel.

There are no right or wrong answers. Do not spend too much time on any one statement but give the answer which seems to describe how you generally feel.

\begin{tabular}{|c|c|c|c|c|}
\hline & $\begin{array}{l}\text { Almost } \\
\text { Never }\end{array}$ & Sometimes & Often & $\begin{array}{l}\text { Almost } \\
\text { Always }\end{array}$ \\
\hline 1. I feel pleasant. & $\overline{\mathrm{A}}$ & $\bar{B}$ & $\mathrm{C}$ & $\mathrm{D}$ \\
\hline 2. I tire quickly. & A & $\mathrm{B}$ & $\mathrm{C}$ & $\mathrm{D}$ \\
\hline 3. I feel like crying. & A & $\mathrm{B}$ & C & $\mathrm{D}$ \\
\hline 4. I wish I could be as happy as others seem to be. & A & $\mathrm{B}$ & C & D \\
\hline $\begin{array}{l}\text { 5. I am losing out on things because I can't make up } \\
\text { my mind soon enough. }\end{array}$ & A & $\mathrm{B}$ & $\mathrm{C}$ & $\mathrm{D}$ \\
\hline 6. I feel rested. & A & B & C & $\mathrm{D}$ \\
\hline 7. I am "calm, cool, and collected". & A & $\mathrm{B}$ & $\mathrm{C}$ & D \\
\hline $\begin{array}{l}\text { 8. I feel that difficulties are piling up so that I } \\
\text { cannot overcome them. }\end{array}$ & A & $\mathrm{B}$ & $\mathrm{C}$ & $\mathrm{D}$ \\
\hline $\begin{array}{l}\text { 9. I worry too much over something that really } \\
\text { doesn't matter. }\end{array}$ & A & $\mathrm{B}$ & $\mathrm{C}$ & $\mathrm{D}$ \\
\hline 10. I am happy. & A & $\mathrm{B}$ & $\mathrm{C}$ & $\mathrm{D}$ \\
\hline 11. I am inclined to take things hard. & A & $\mathrm{B}$ & $\mathrm{C}$ & $\mathrm{D}$ \\
\hline 12. I lack self-confidence. & A & B & C & D \\
\hline 13. I feel secure. & A & $\mathrm{B}$ & C & D \\
\hline 14. I try to avoid facing a crisis or difficulty. & A & $\mathrm{B}$ & C & $\mathrm{D}$ \\
\hline 15. I feel blue. & A & B & C & D \\
\hline 16. I am content. & $\mathrm{A}$ & $\mathrm{B}$ & $\mathrm{C}$ & $\mathrm{D}$ \\
\hline $\begin{array}{l}\text { 17. Some unimportant thought runs through my } \\
\text { mind and bothers me. }\end{array}$ & A & $\mathrm{B}$ & C & $\mathrm{D}$ \\
\hline $\begin{array}{l}\text { 18. I take disappointments so keenly that I can't put } \\
\text { hem out of my mind. }\end{array}$ & A & $\mathrm{B}$ & $\mathrm{C}$ & $\mathrm{D}$ \\
\hline 19. I am a steady person. & A & $\mathrm{B}$ & $\mathrm{C}$ & $\mathrm{D}$ \\
\hline $\begin{array}{l}\text { 20. I get in a state of tension or turmoil as I think } \\
\text { over my recent concerns and interests. }\end{array}$ & A & $\mathrm{B}$ & C & $\mathrm{D}$ \\
\hline
\end{tabular}


Navn:

Type hopp: Training / Konkurranse
Land:

Runde:
Dato:

Gjentagelse? Ja / Nei

\section{Vennligst fyll ut denne siden rett etter ditt hopp.}

Nedenfor er noen utsagn fra personer om hvordan de føler det når de konkurrerer i sport. Les hvert utsagn og marker bokstav ved svar som best beskriver hvordan du følte det mens du satt i flyet for ditt siste hopp.

Det er ingen riktige eller gale svar. Bruk ikke for lang tid på noen utsagn. Husk å velge utsagnet som best beskriver hvordan du følte det under flyturen opp til utspringshøyden på ditt siste hopp.

\begin{tabular}{|c|c|c|c|c|}
\hline & $\begin{array}{c}\text { Koverhodet } \\
\text { ikke }\end{array}$ & Litt & Temmelig & Svært \\
\hline 1. Jeg er litt bekymret for neste hopp. & $\mathrm{A}$ & $\mathrm{B}$ & C & $\mathrm{D}$ \\
\hline 2. Jeg er nervøs. & A & B & C & D \\
\hline $\begin{array}{l}\text { 3. Jeg føler meg fullstendig avslappet og } \\
\text { relaxed. }\end{array}$ & A & B & C & $\mathrm{D}$ \\
\hline 4. Jeg tviler på meg selv. & A & B & C & $\mathrm{D}$ \\
\hline 5. Jeg făr den store skjelven. & A & B & C & $\mathrm{D}$ \\
\hline 6. Jeg føler meg som situasjonens herre. & A & B & C & $\mathrm{D}$ \\
\hline $\begin{array}{l}\text { 7. Jeg er redd for at jeg ikke kan være sà } \\
\text { god som jeg er i denne konkurransen. }\end{array}$ & A & B & $\mathrm{C}$ & $\mathrm{D}$ \\
\hline 8. Kroppen min er spent. & A & B & C & $\mathrm{D}$ \\
\hline 9. Jeg er selvsikker. & A & B & C & $\mathrm{D}$ \\
\hline 10. Jeg er redd for å tape. & A & B & C & $\mathrm{D}$ \\
\hline 11. Jeg har en rar følelse i magen. & A & B & C & $\mathrm{D}$ \\
\hline 12. Jeg føler meg sikker. & A & B & C & $\mathrm{D}$ \\
\hline $\begin{array}{l}\text { 13. Jeg er redd for brainlock under } \\
\text { konkurransen. }\end{array}$ & $\mathrm{A}$ & B & $\mathrm{C}$ & $\mathrm{D}$ \\
\hline 14. Kroppen min føles avslappet. & A & B & C & $\mathrm{D}$ \\
\hline $\begin{array}{l}\text { 15. Jeg er sikker pà at jeg greier } \\
\text { utfordringen. }\end{array}$ & A & B & $\mathrm{C}$ & D \\
\hline 16. Jeg er redd for à vise et dårlig hopp. & A & B & C & $\mathrm{D}$ \\
\hline 17. Hjertet mitt raser. & A & B & C & $\mathrm{D}$ \\
\hline 18. Jeg er sikker på at jeg giør det bra. & A & $\mathrm{B}$ & C & $\mathrm{D}$ \\
\hline 19. Jeg lurer på om jeg når mine măl. & A & B & C & $\mathrm{D}$ \\
\hline 20. Jeg får hjertet $i$ halsen. & A & B & C & $\mathrm{D}$ \\
\hline 21. Mentalt er jeg avslappet. & A & B & C & $\mathrm{D}$ \\
\hline $\begin{array}{l}\text { 22. Jeg er redd for at andre blir skuffet } \\
\text { over min prestasjon. }\end{array}$ & A & B & $\mathrm{C}$ & $\mathrm{D}$ \\
\hline 23. Hendene mine er fuktige. & A & B & C & $\mathrm{D}$ \\
\hline $\begin{array}{l}\text { 24. Jeg føler meg sikker da jeg har } \\
\text { forestilt meg mentalt hvordan jeg kan } \\
\text { nå mine mål. }\end{array}$ & A & B & C & $\mathrm{D}$ \\
\hline $\begin{array}{l}\text { 25. Jeg er redd for at jeg ikke kan } \\
\text { konsentrere meg nok. }\end{array}$ & $\mathrm{A}$ & B & C & $\mathrm{D}$ \\
\hline $\begin{array}{l}\text { 26. Kropper min føles stiv og } \\
\text { forkrampet. }\end{array}$ & A & B & C & $\mathrm{D}$ \\
\hline $\begin{array}{l}\text { 27. Jeg er sikker pà at jeg kan utholde } \\
\text { presset. }\end{array}$ & $\mathrm{A}$ & B & C & $\mathrm{D}$ \\
\hline
\end{tabular}

Gå til neste side (generell spørsmål) når du har tid. 


\begin{tabular}{|lll}
\hline Navn: $\ldots$ Land: $\ldots$ Dato: \\
\hline
\end{tabular}

\section{Vennligst gi noen generell informasjon.}

Røker: Ja Nei

Antall hopp:

Frittfall tid:

Kjønn: Mann Kvinne

FS hopp:

Vind tunnel tid:

Antall år i sport:

Vennligst førklar rutinen for ditt lag før flyturen opp til utspringshøyden (kort):

Kommentarer:

Jeg vil gjerne motta en kopi av mitt testresultat via E-Post eller fax: Ja Nei

Hvis Ja: E-Post: Fax:

\section{Tusen takk for ditt samarbeid.}

Kun for oss:

\begin{tabular}{|c|c|c|}
\hline Temperatur: & Luftfeuchtigkeit: & Luftdruck: \\
\hline Polartesternr.: & Speicher Nr.: & Dateiname: \\
\hline Nullzeit: $\_$___ & & persönliche Note: \\
\hline Flugzeug Nr.: & Team Nr.: 12 & Take-Off: ___ \\
\hline Run-In $1^{\text {st }}: \ldots$ & Standby $1^{\text {st }}: \ldots$ & Exit $1^{\text {st }}: \ldots$ \\
\hline Run-In $2^{\text {nd }}: \ldots$ & Standby $2^{\text {nd }}: \ldots$ & Exit $2^{\text {nd. }}:[\quad[\quad[-$ \\
\hline Schirmöffnung: ___ & Landung: ___ : _ : & _Ruhepuls: \\
\hline
\end{tabular}


Nombre:

Nacionalidad:

Fecha:

Tipo de salto: Entrenamiento / Concurso Asalto número.:

Repeater? Sí / No

\section{Por favor, contesta a las preguntas en seguida del salto.}

A bajo se presentan 27 afirmaciones de deportistas sobre sus sentimientos antes de un concurso. Lee estas afirmaciones y marca la letra que describe lo más tus sentimientos en el momento en el que estuviste en el avión para hacer tu último salto en paracíadas.

No hay contestaciones correctas o falsos en este cuestionario. No piensa demasiado en las afirmaciones, pero eliga la que describe tus sentimientos antes del último salto.

\begin{tabular}{|c|c|c|c|c|}
\hline & $\begin{array}{c}\text { De ningún } \\
\text { modo }\end{array}$ & Un poco & Bastante & Mucho \\
\hline 1. Estoy preocupandome del salto. & $\mathrm{A}$ & $\mathrm{B}$ & $\mathrm{C}$ & $\mathrm{D}$ \\
\hline 2. Estoy nervioso. & A & B & $\mathrm{C}$ & D \\
\hline 3. Estoy relajada. & A & B & C & D \\
\hline 4. Tengo dudas en mi mismo. & $\mathrm{A}$ & B & $\mathrm{C}$ & $\mathrm{D}$ \\
\hline 5. Noto un poco de pánico. & A & $\mathrm{B}$ & C & D \\
\hline 6. Me siento dueño de la situación. & A & B & C & $\mathrm{D}$ \\
\hline $\begin{array}{l}\text { 7. Estoy preocupado de que no suceda según } \\
\text { mis posibilidades. }\end{array}$ & A & B & $\mathrm{C}$ & $\mathrm{D}$ \\
\hline 8. Mi cuerpo está tenso." & A & B & $\mathrm{C}$ & D \\
\hline 9. Estoy seguro de mi mismo. & A & B & C & D \\
\hline 10. Tengo miedo de perder. & A & B & $\mathrm{C}$ & D \\
\hline 11. Tengo un sentimiento de defallecimiento. & A & B & $\mathrm{C}$ & D \\
\hline 12. Me siento seguro. & A & B & C & D \\
\hline $\begin{array}{l}\text { 13. Estoy preocupado de que pueda conseguir } \\
\text { "brainlock" bajo la presión del concurso. }\end{array}$ & A & B & $\mathrm{C}$ & $\mathrm{D}$ \\
\hline 14. Mi cuerpo se siente relajado. & A & B & C & $\mathrm{D}$ \\
\hline $\begin{array}{l}\text { 15. Estoy seguro de que puedo vencer el } \\
\text { desafío. }\end{array}$ & $\mathrm{A}$ & B & $\mathrm{C}$ & $\mathrm{D}$ \\
\hline 16. Tengo miedo de hacer un mal salto. & A & B & $\mathrm{C}$ & $\mathrm{D}$ \\
\hline 17. Mi corazón palpita fuertemente. & A & B & C & $\mathrm{D}$ \\
\hline $\begin{array}{l}\text { 18. Estoy seguro de que doy un buen } \\
\text { rendimiento. }\end{array}$ & $\mathrm{A}$ & B & $\mathrm{C}$ & $\mathrm{D}$ \\
\hline $\begin{array}{l}\text { 19. Estoy preocupado de no alcanzar mis } \\
\text { metas. }\end{array}$ & A & B & $\mathrm{C}$ & $\mathrm{D}$ \\
\hline 20. Se me cae el alma a los pies. & A & B & $\mathrm{C}$ & $\mathrm{D}$ \\
\hline 21. Mentalmente estoy relajado. & A & B & $\mathrm{C}$ & D \\
\hline $\begin{array}{l}\text { 22. Tengo miedo de que otros puedan estar } \\
\text { desilusionado de mi rendimiento. }\end{array}$ & A & B & $\mathrm{C}$ & $\mathrm{D}$ \\
\hline 23. Mis manos están mojados. & A & B & C & $\mathrm{D}$ \\
\hline $\begin{array}{l}\text { 24. Estoy lleno de confianza, porque me había } \\
\text { imaginado el alcanzo de mis metas } \\
\text { mentalmente. }\end{array}$ & A & B & $\mathrm{C}$ & $\mathrm{D}$ \\
\hline $\begin{array}{l}\text { 25. Estoy preocupado de no poder } \\
\text { concentrarme bastante. }\end{array}$ & A & B & $\mathrm{C}$ & $\mathrm{D}$ \\
\hline 26. Mi cuerpo se siente crispado. & A & B & C & D \\
\hline 27. Estoy seguro de resistar al la presión. & A & $\mathrm{B}$ & $\mathrm{C}$ & $\mathrm{D}$ \\
\hline
\end{tabular}

Cuando tienes tiempo por favor, contesta a las preguntas de la siguiente página (preguntas generales). 


\begin{tabular}{|lll}
\hline Nombre: _ Nacionalidad: __ Fecha: & \\
\hline
\end{tabular}

\section{Por favor, da un poco de información sobre tú mismo.}

Fumador: Sí / No Sexo: m / f Edad:

Talla:

Peso:

Cabtidad de saltos:

de estos saltos 'FS':

Años en deporte:

Tiempo de salto libre:

Tiempo de túnel de viento:

Posición de salida:

Por favor, explica brevemente la rutina de tu equipo en el avión en preparación para un salto.

Otros comentarios:

Quería una copia de mis resultados via e-mail o fax: Sí / No

En caso de sí: E-Mail: Fax:

Muchas gracias por tu colaboración.

Solo para la persona que evalua:

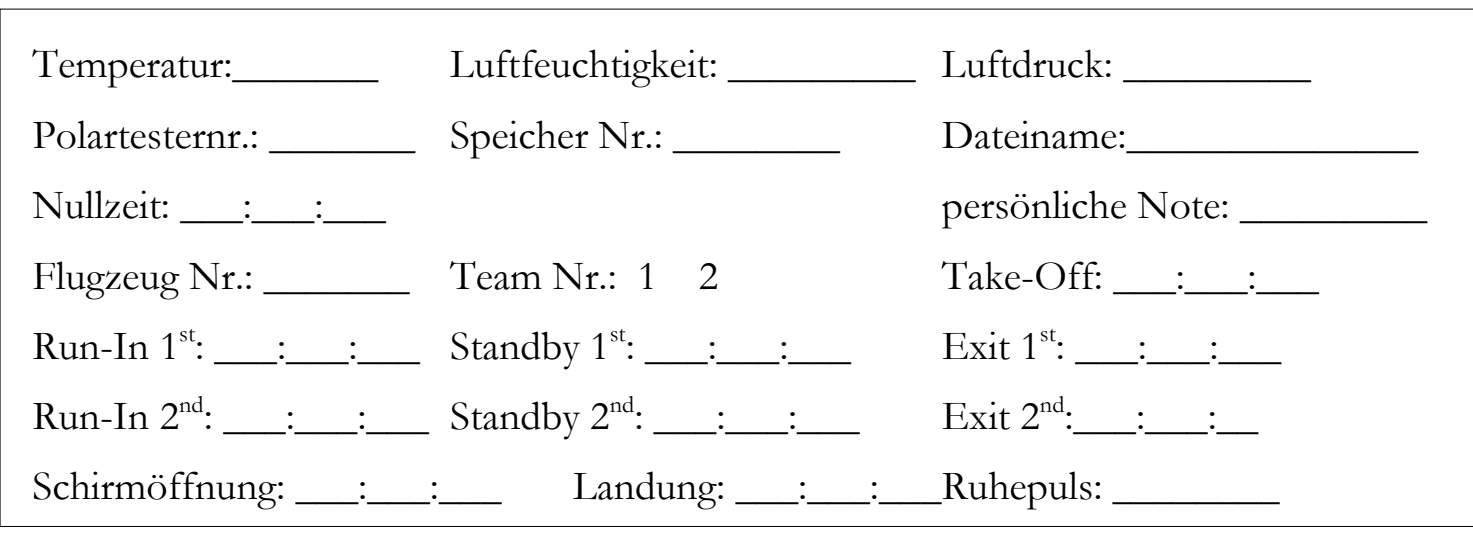




\section{Ausgabe GEMCAT II: Spitzenkatastrophe für niedrige Ängstlichkeit}

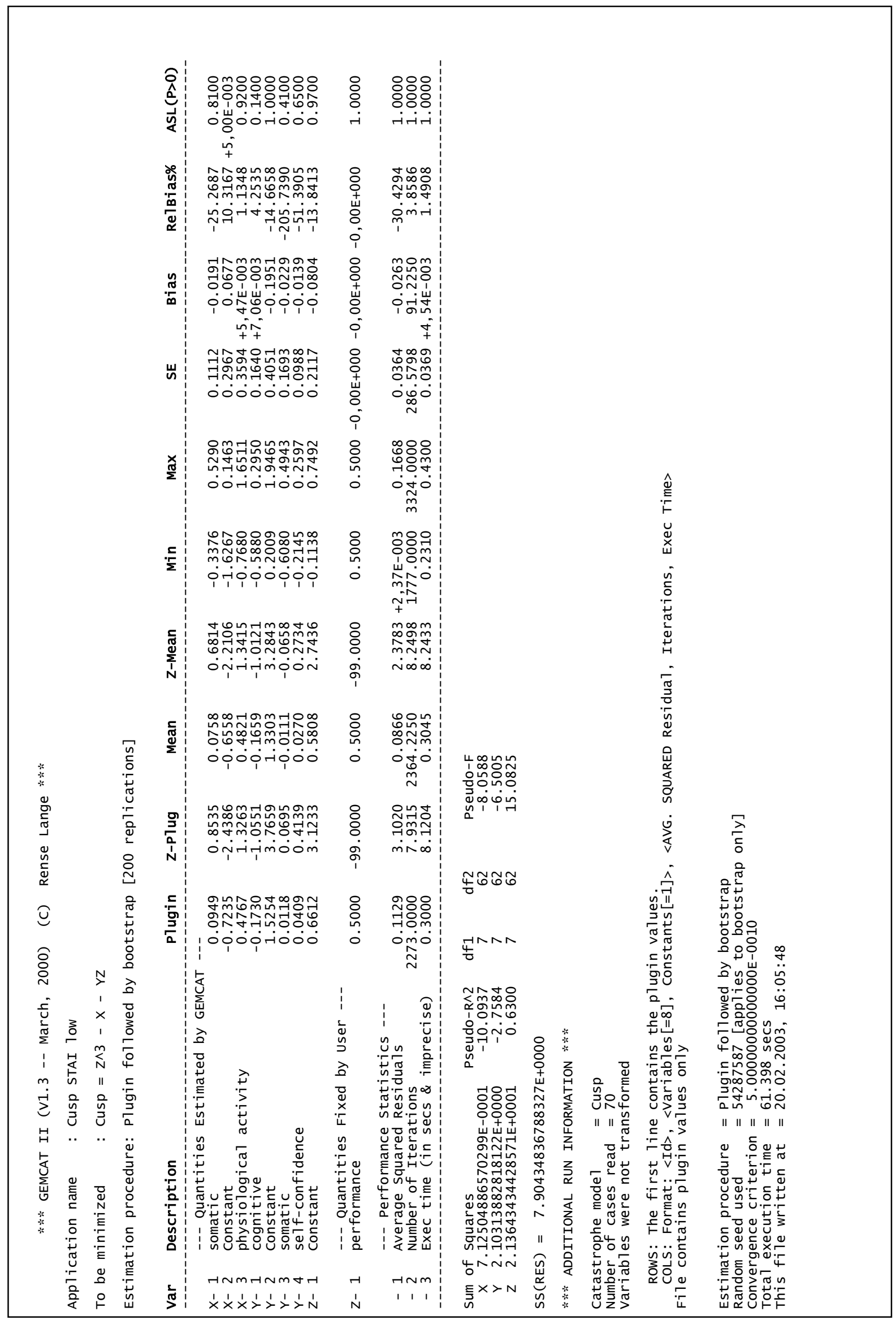




\section{Ausgabe GEMCAT II: Spitzenkatastrophe für mittlere Ängstlichkeit}

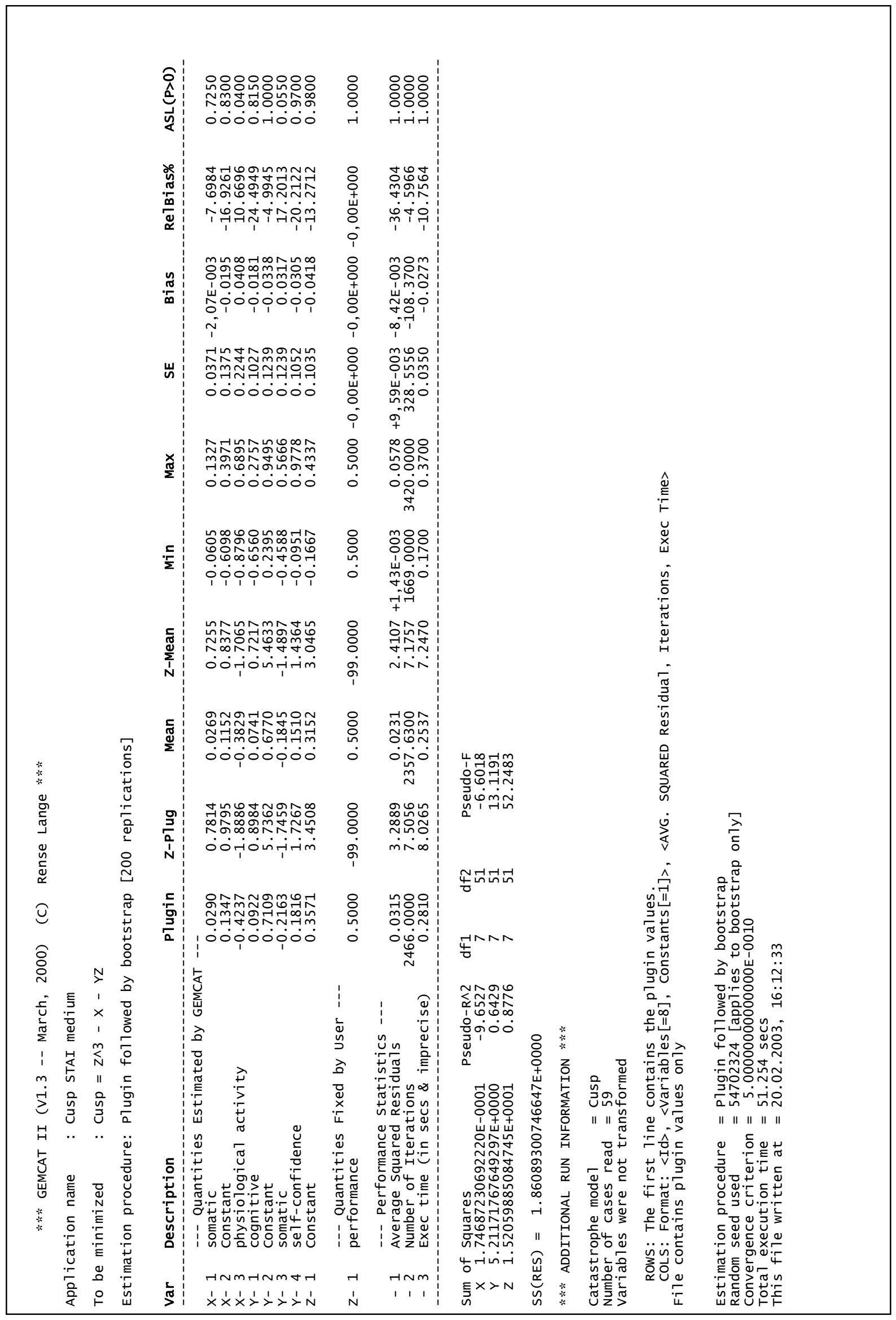




\section{Ausgabe GEMCAT II: Spitzenkatastrophe für hohe Ängstlichkeit}

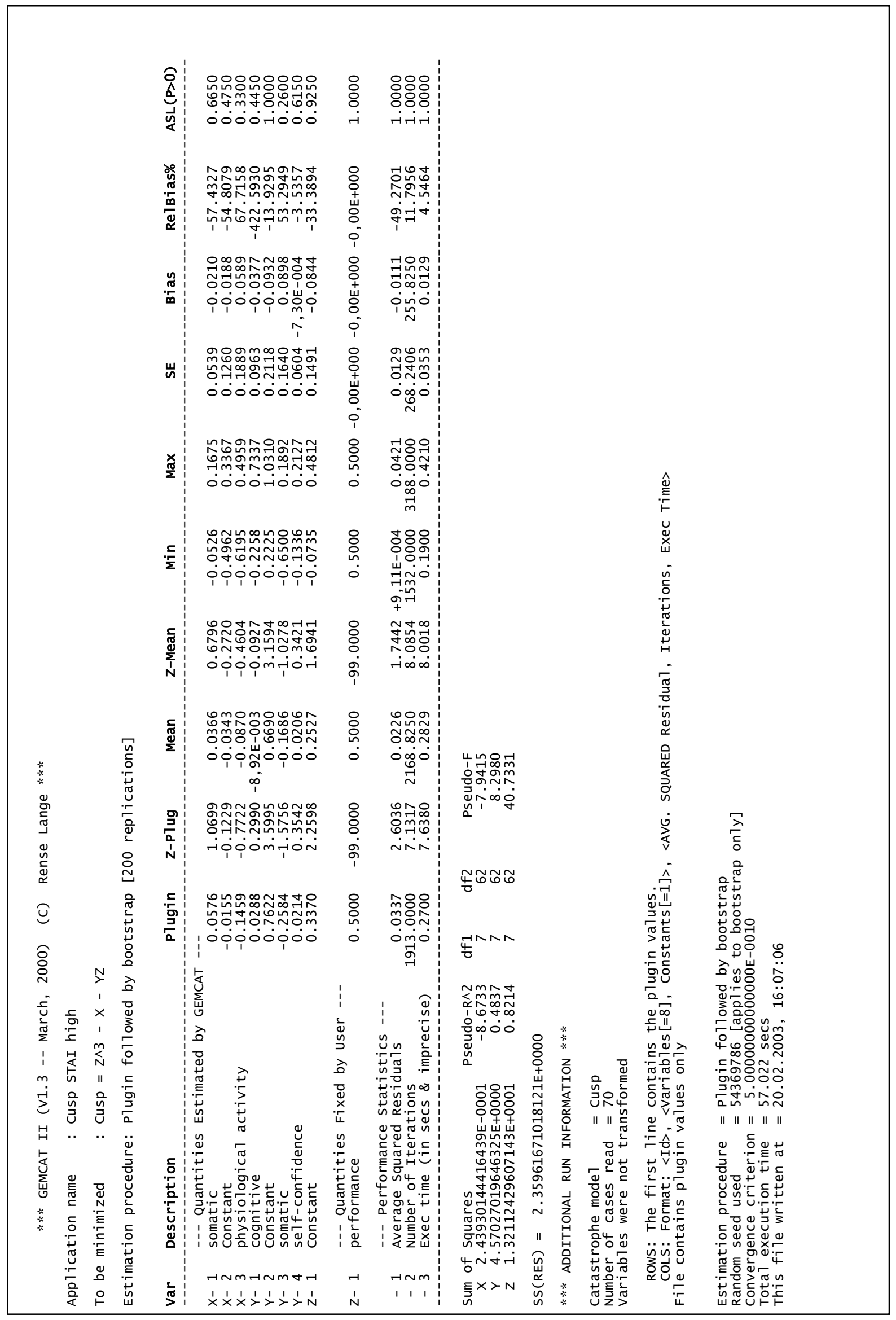




\section{Ausgabe GEMCAT II: Spitzenkatastrophe für niedrige Selbstwirksamkeit}

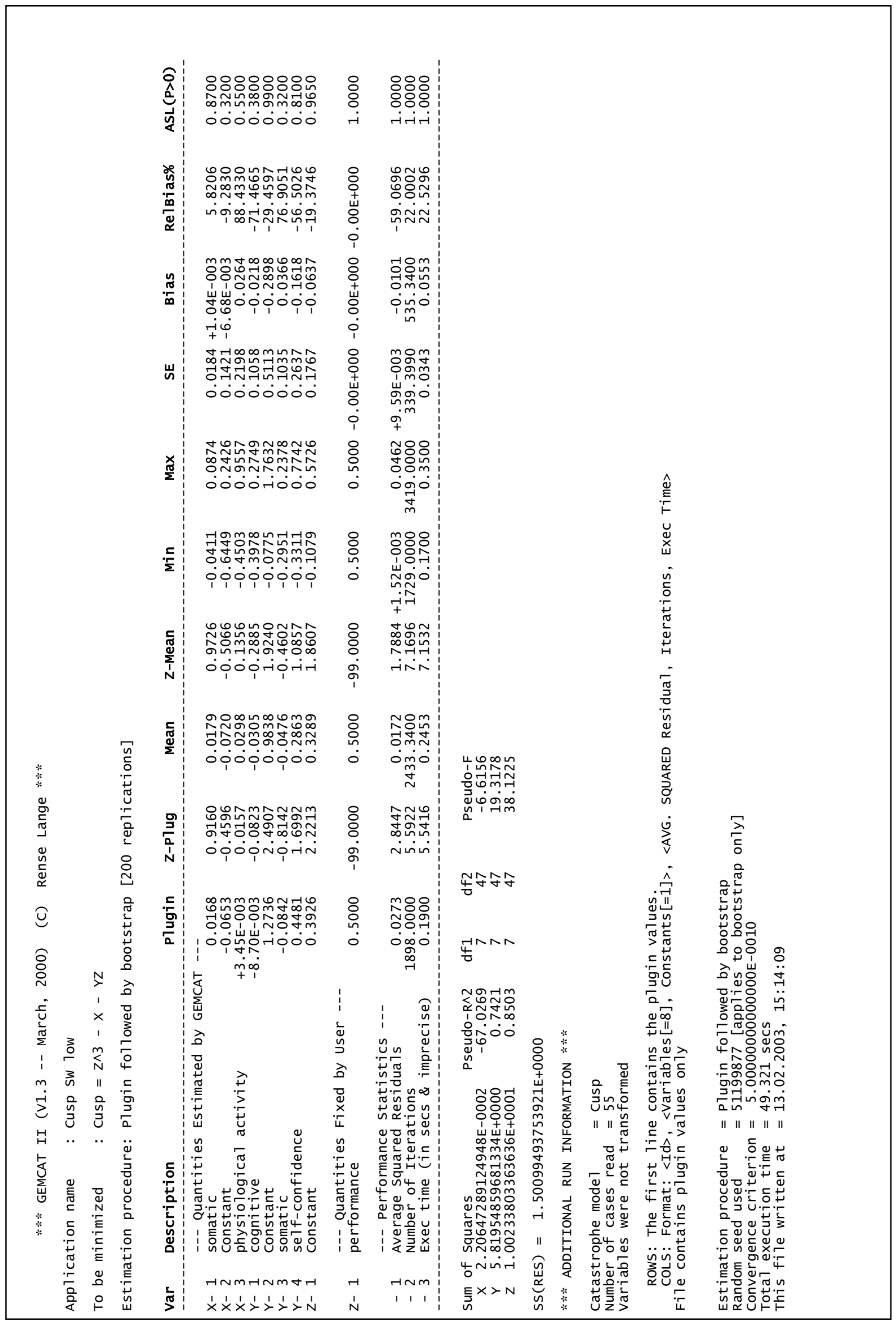




\section{Ausgabe GEMCAT II: Spitzenkatastrophe für mittlere Selbstwirksamkeit}

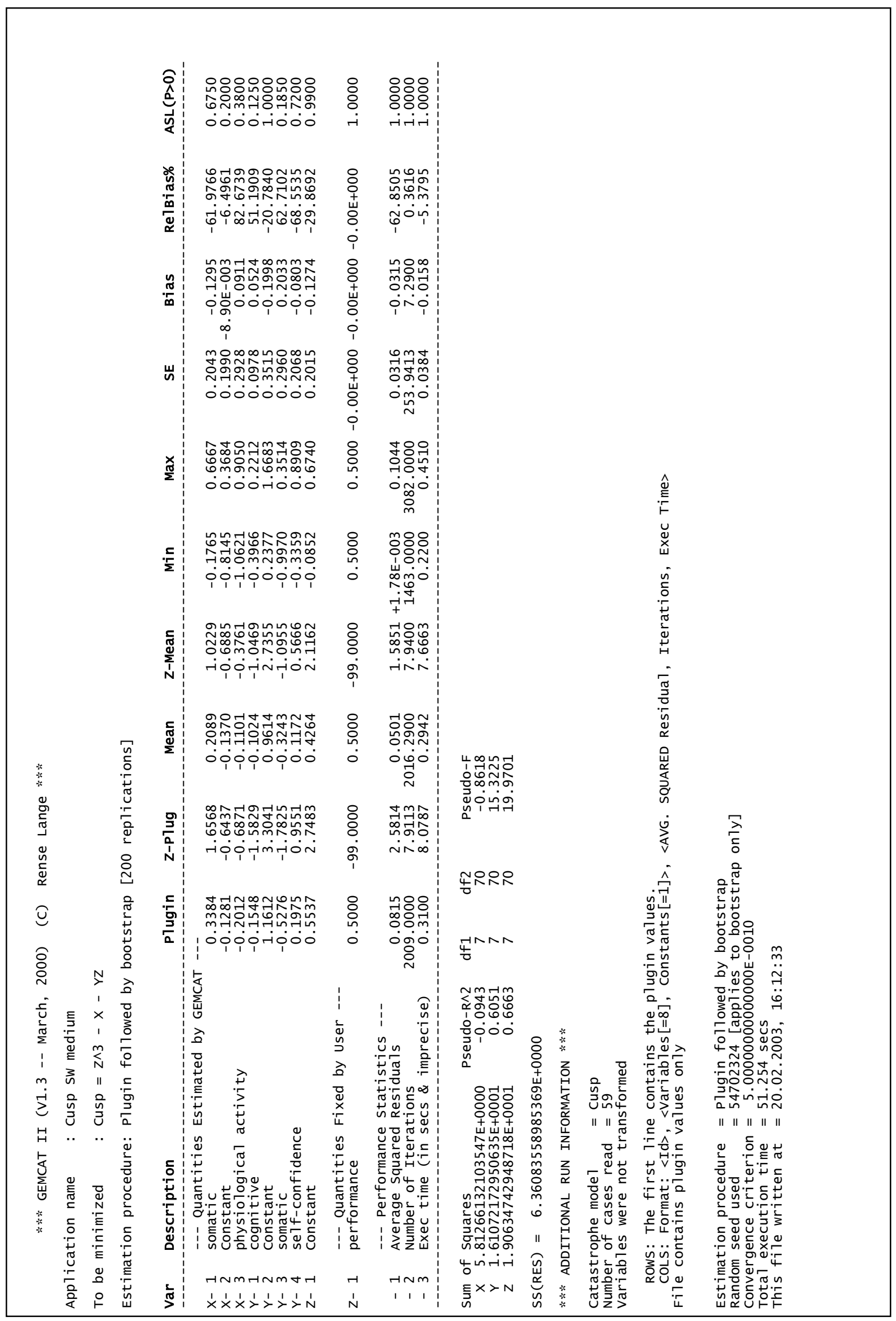




\section{Ausgabe GEMCAT II: Spitzenkatastrophe für hohe Selbstwirksamkeit}

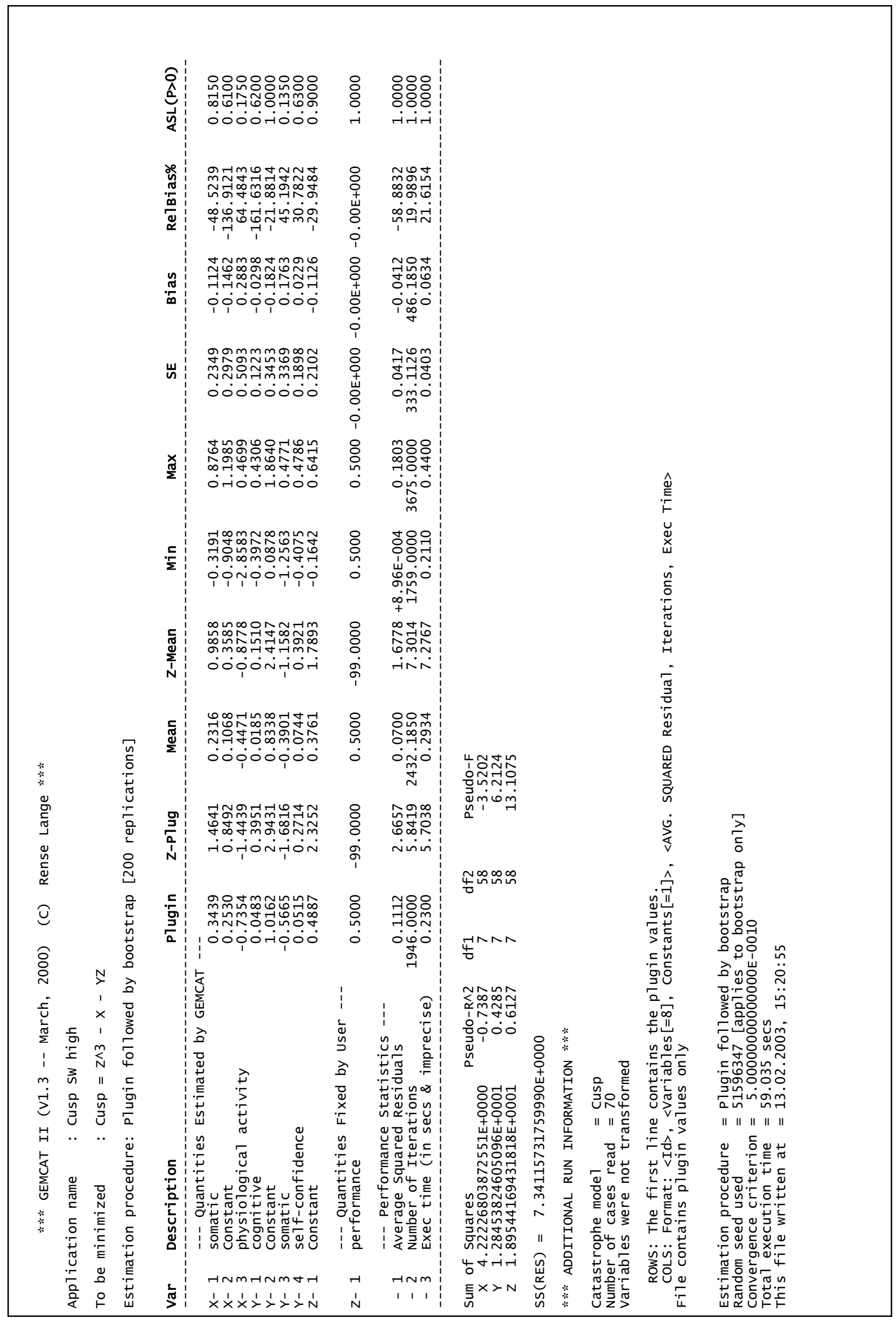




\section{Ausgabe GEMCAT II: Spitzenkatastrophe für geringe Sprungerfahrung}

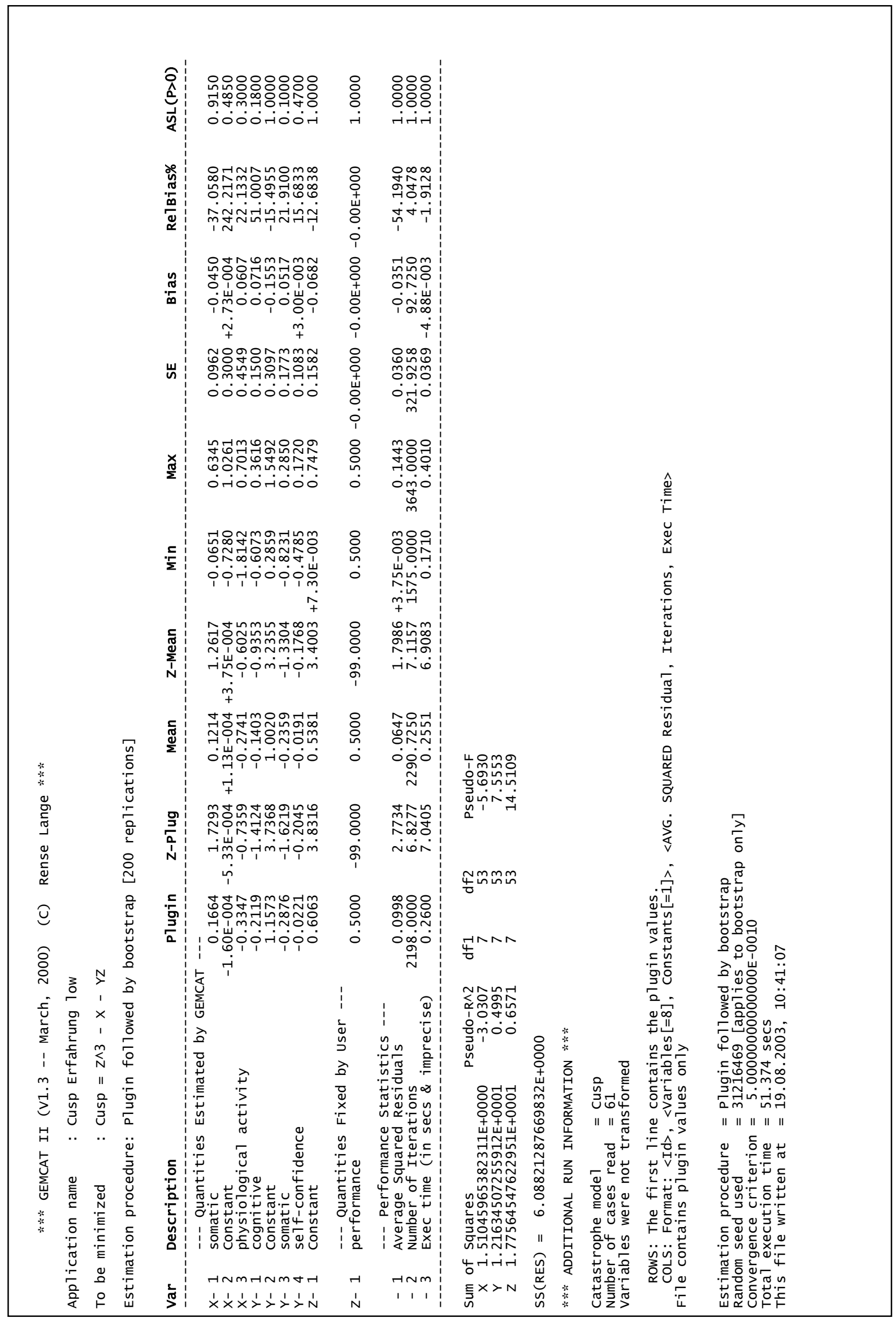




\section{Ausgabe GEMCAT II: Spitzenkatastrophe für mittlere Sprungerfahrung}

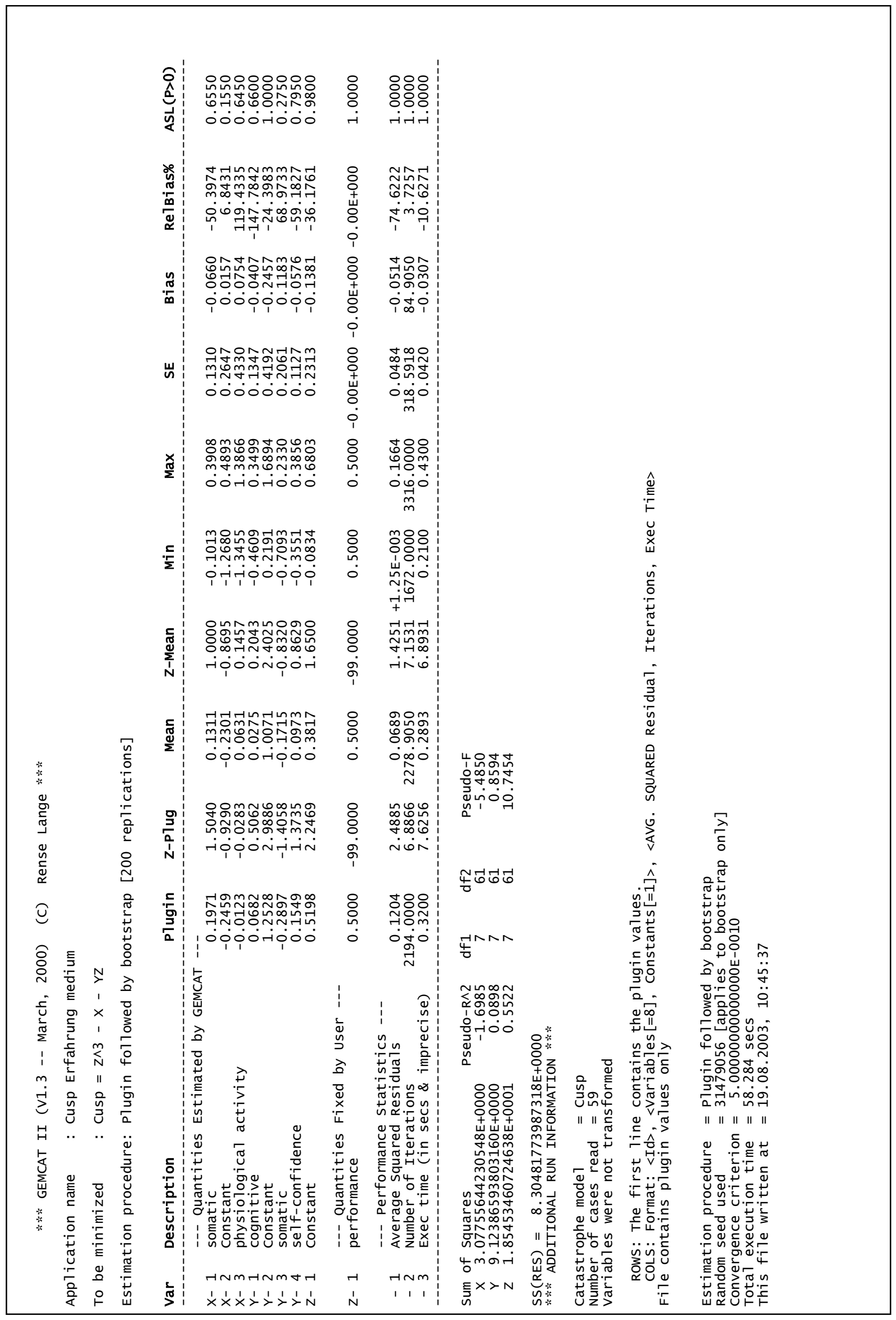




\section{Ausgabe GEMCAT II: Spitzenkatastrophe für hohe Sprungerfahrung}

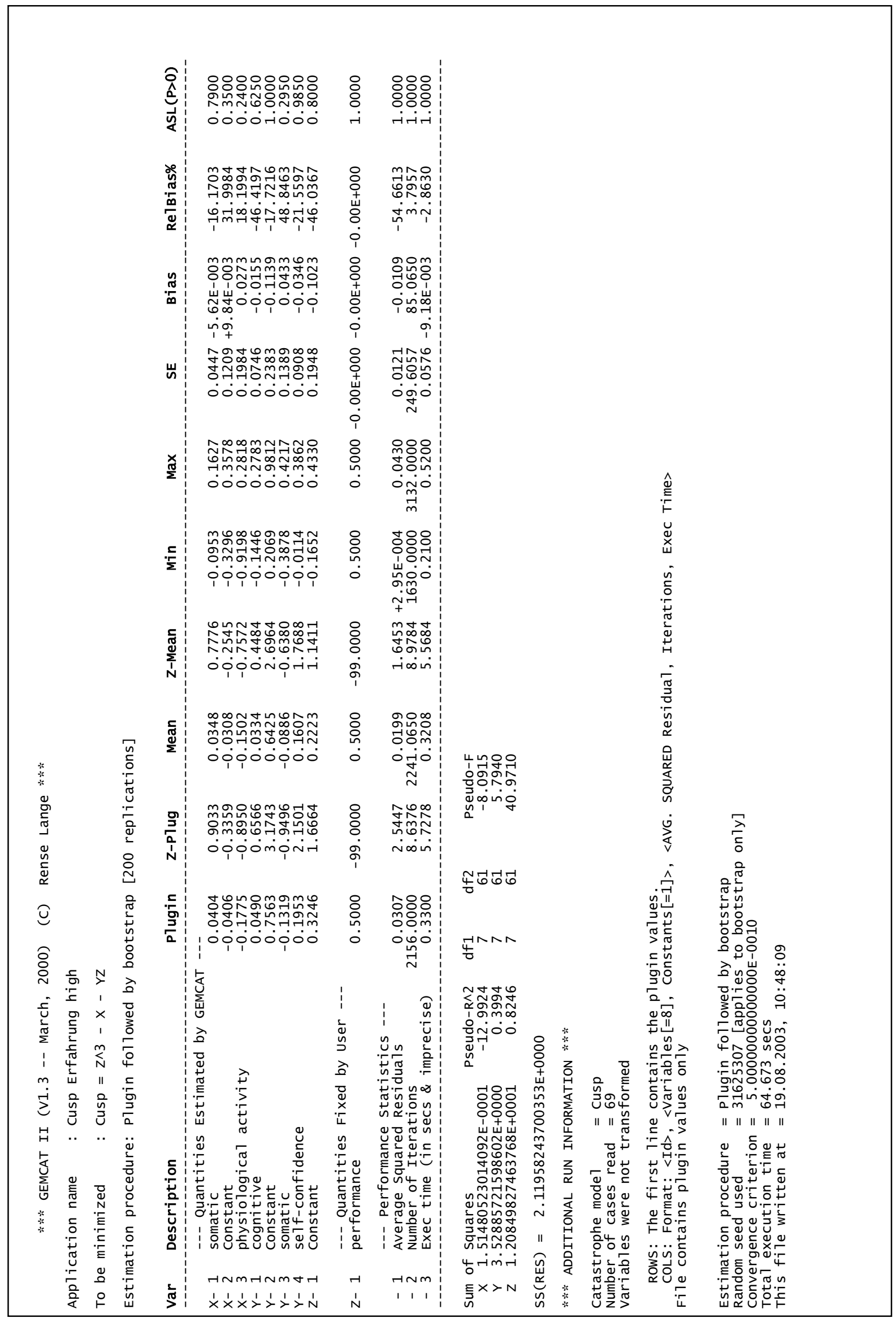




\section{Ausgabe GEMCAT II: Schmetterlingskatastrophe für niedrige Ängstlichkeit}

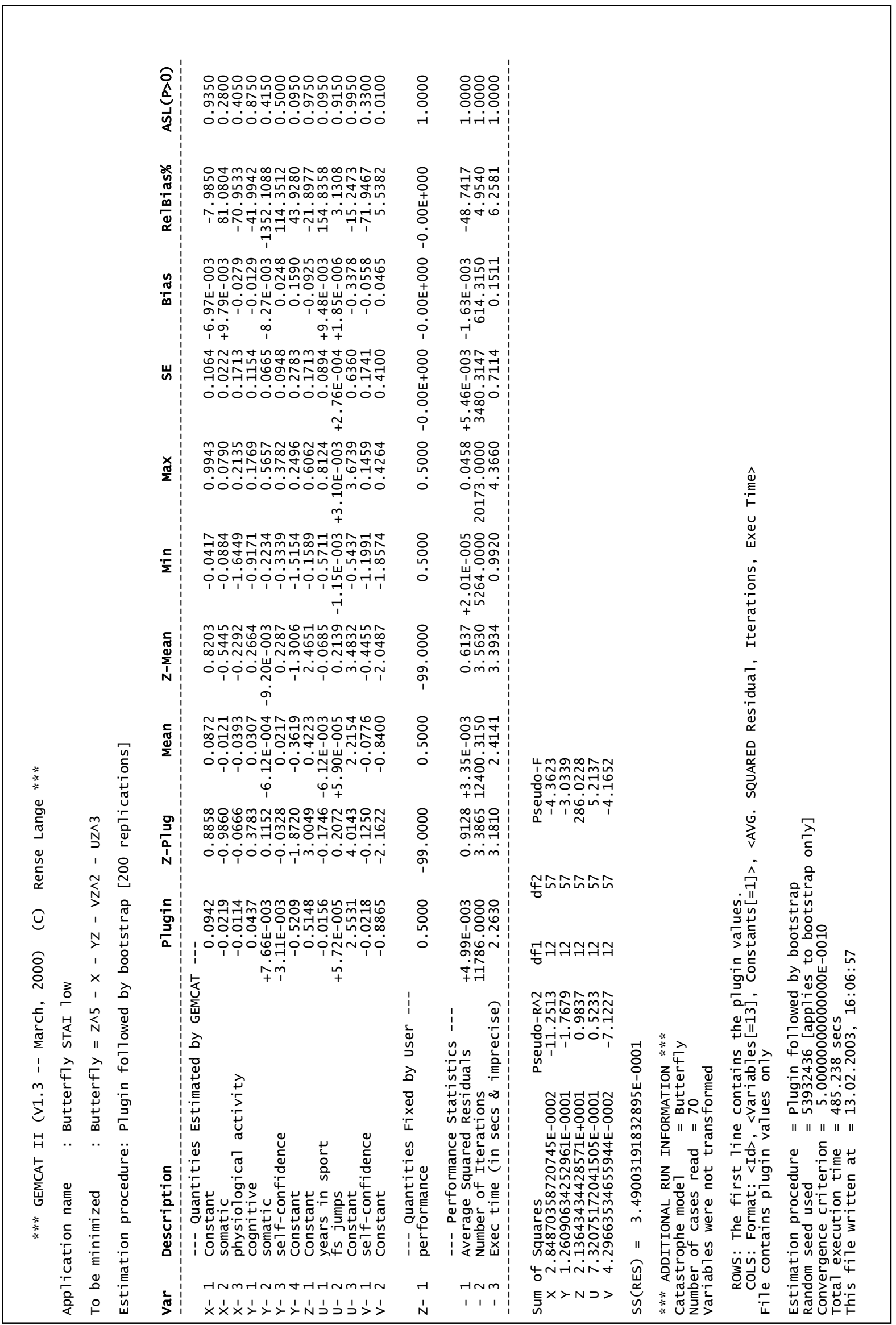




\section{Ausgabe GEMCAT II: Schmetterlingskatastrophe für mittlere Ängstlichkeit}

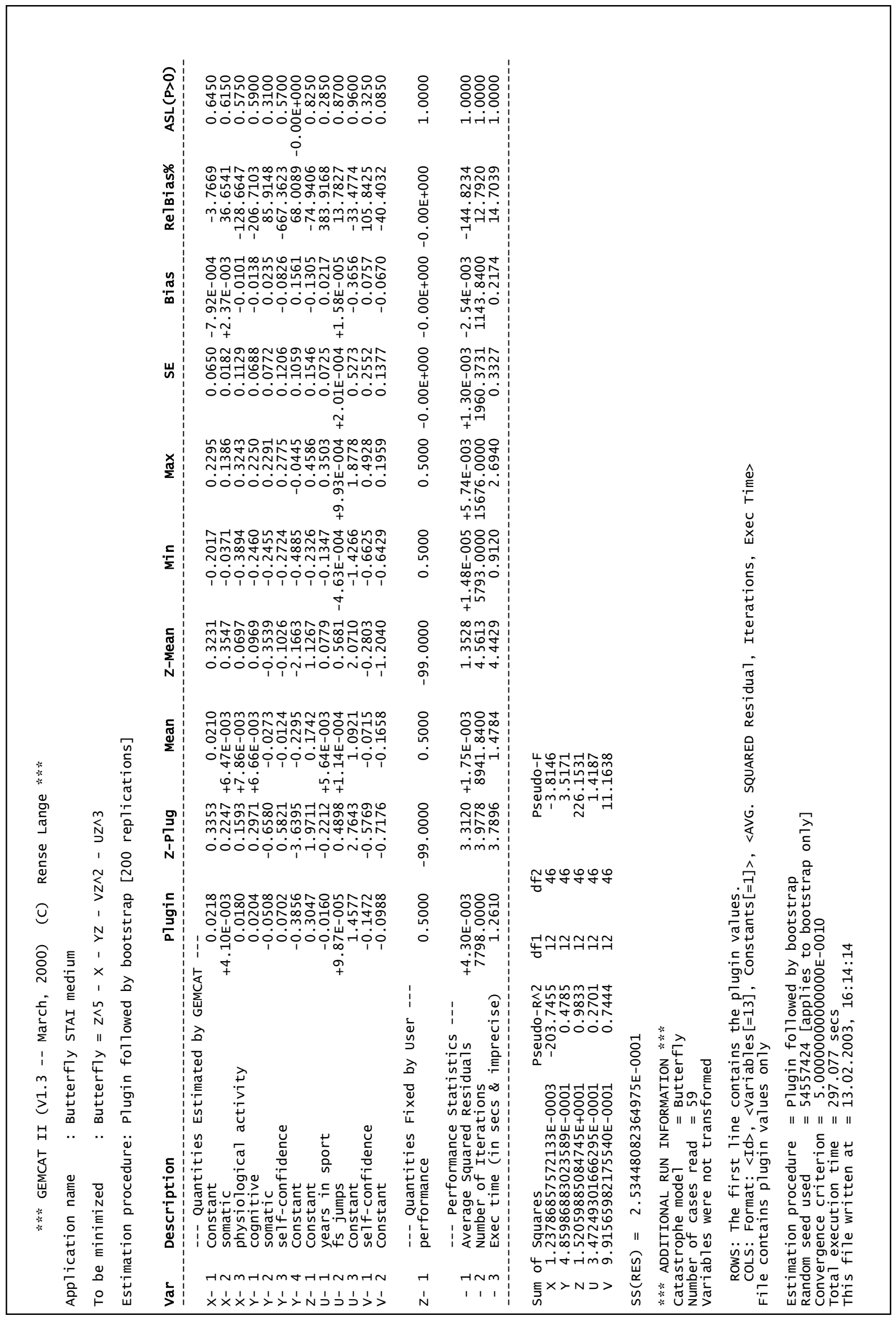




\section{Ausgabe GEMCAT II: Schmetterlingskatastrophe für hohe Ängstlichkeit}

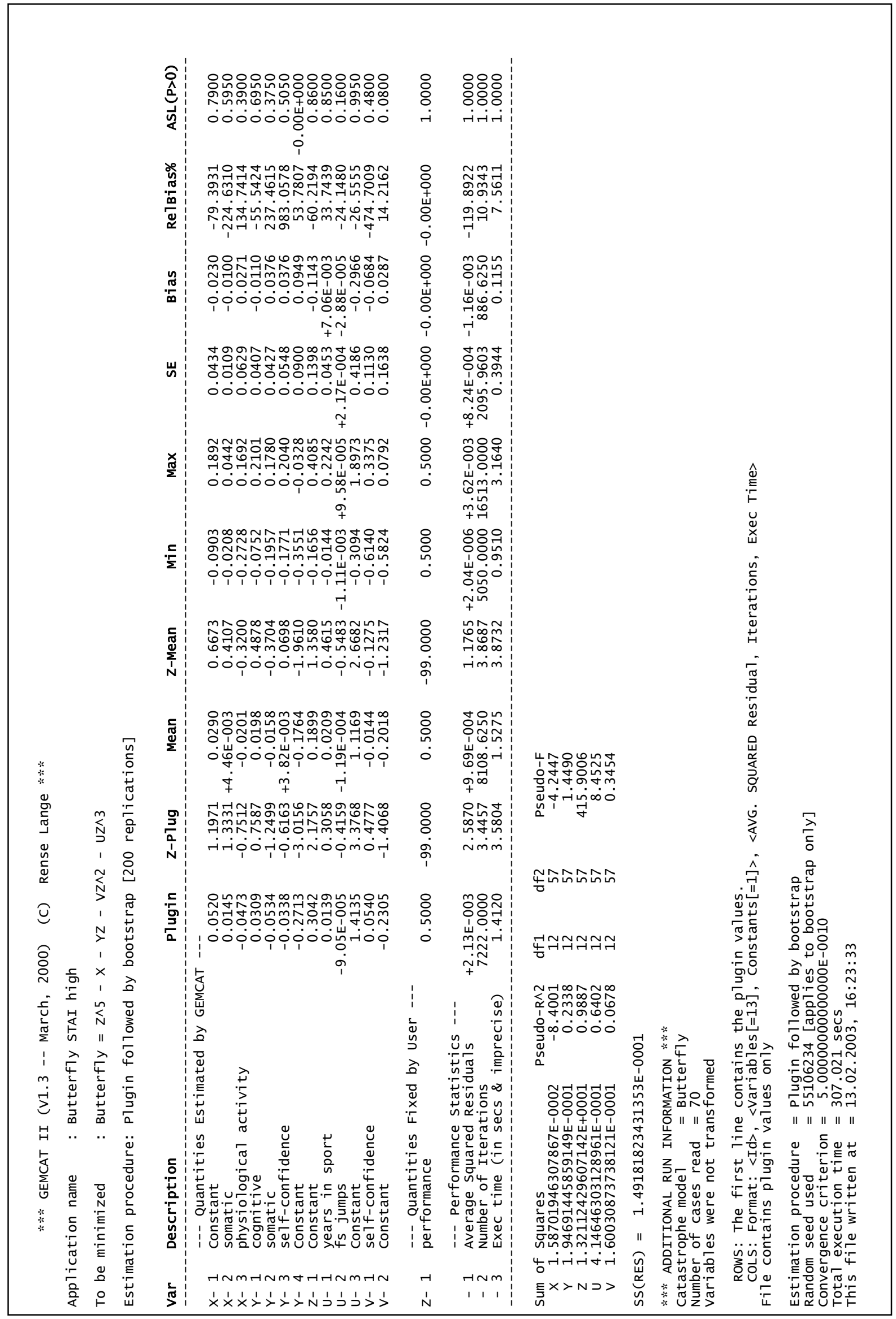




\section{Freifallformationsprogramm 4er-Disziplin}

4er: Blockübergänge 1-8

(INTER)

(INTER)


4er: Blockübergänge 9-16

(INTER

(INTER)


4er: Blockübergänge 17-22

(INTER

(INTER)


4er: Freie Formationen

(1)




\section{Freifallformationsprogramm 8er-Disziplin}

8er: Blockübergänge 1-8

(INTER)

(INTER


8er: Blockübergänge 9-16

(INTER)

(INTER)


8er: Blockübergänge 17-22

(INTER)

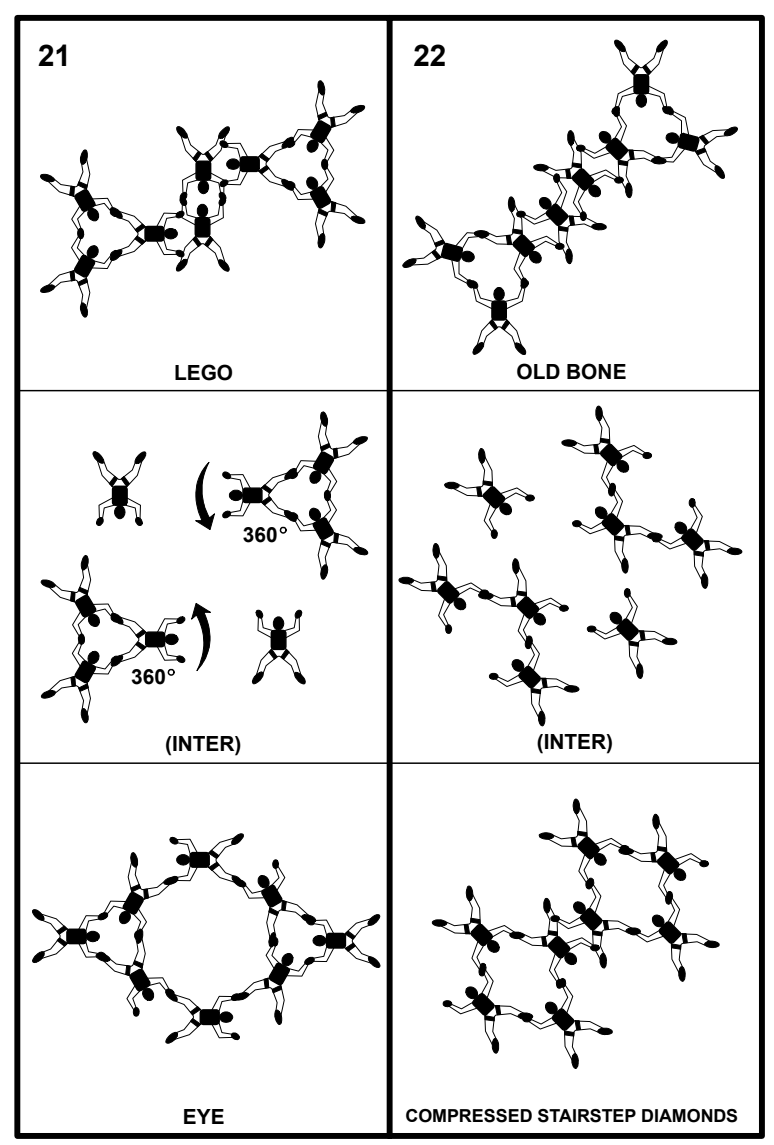


8er: Freie Formationen

(1)




\section{Lebenslauf}

Persönliche Daten:

Hochschulreife:

Wehrdienst:

Studium:

Promotion:

Praktika/Nebentätigkeiten:

Lehrtätigkeiten:

Sportliche Erfolge:
Steffen Schiedek, Bevenroder Str. 44, 38108 Braunschweig, Tel.: 0531-3104161, geboren am 08.04.1971 in Göttingen, ledig

Abitur am Otto-Hahn-Gymnasium in Göttingen im Mai 1990

Juli 1990 bis Juni 1992 als Zeitsoldat in der Fernspähkompanie 100 in Braunschweig, zur Zeit Leutnant der Reserve

1993-1995 Studium Mathematik, Physik an der Georg-AugustUniversität Göttingen

1995-2002 Studium Physik, Sport, Erweiterungsfach Informatik an der Georg-August-Universität Göttingen

Studienschwerpunkte in den Bereichen Biomechanik, Wasser- und Erlebnissport, Sportpsychologie

Juni 2002 Erstes Staatsexamen in Physik, Sport

Juni 2004 an der Sozialwissenschaftlichen Fakultät der GeorgAugust-Universität Göttingen, Gesamtnote: magna cum laude

1993-2003 Lehrtätigkeit als Fallschirmsprunglehrer und als Trainer Freifallformationsspringen

1997-2002 Lehrtätigkeit als studentische Hilfskraft am Institut für Sportwissenschaften in den Bereichen Kanu, Rudern, naturwissenschaftliche Grundlagen, Erstellung von Lehrvideos

seit November 2002 Studienreferendar am Studienseminar Wolfsburg für das Lehramt an Gymnasien

seit 1994 Mitglied der Deutschen Nationalmannschaft im AchterFreifallformationsspringen

1994 EM Achter-Freifallformation in Spanien: 3. Platz

1997 WM Achter-Freifallformation in der Türkei: 4. Platz

seit 1996 Deutscher Meister Achter-Freifallformation,

2002 Vizemeister Deutschland-Cup Vierer-Freifallformation 


\section{Eidesstattliche Erklärung}

Ich versichere, dass ich die eingereichte Dissertation „Angst und Leistung im Rahmen der Katastrophentheorie - Untersuchungen zum optimalen Erregungsniveau bei Fallschirmspringern“" selbständig und ohne unerlaubte Hilfsmittel verfasst habe. Anderer als der von mir angegebenen Hilfsmittel und Schriften habe ich mich nicht bedient. Alle wörtlich oder sinngemäß den Schriften anderer Autoren entnommenen Stellen habe ich kenntlich gemacht.

Steffen Schiedek 